\title{
Mechanisms of action of atrial-specific anti-arrhythmic drugs
}

Citation for published version (APA):

Sobota, V. (2021). Mechanisms of action of atrial-specific anti-arrhythmic drugs. [Doctoral Thesis, Maastricht University]. Maastricht University. https://doi.org/10.26481/dis.20210528vs

Document status and date:

Published: 01/01/2021

DOI:

10.26481/dis.20210528vs

Document Version:

Publisher's PDF, also known as Version of record

\section{Please check the document version of this publication:}

- A submitted manuscript is the version of the article upon submission and before peer-review. There can be important differences between the submitted version and the official published version of record.

People interested in the research are advised to contact the author for the final version of the publication, or visit the DOI to the publisher's website.

- The final author version and the galley proof are versions of the publication after peer review.

- The final published version features the final layout of the paper including the volume, issue and page numbers.

Link to publication

\footnotetext{
General rights rights.

- You may freely distribute the URL identifying the publication in the public portal. please follow below link for the End User Agreement:

www.umlib.nl/taverne-license

Take down policy

If you believe that this document breaches copyright please contact us at:

repository@maastrichtuniversity.nl

providing details and we will investigate your claim.
}

Copyright and moral rights for the publications made accessible in the public portal are retained by the authors and/or other copyright owners and it is a condition of accessing publications that users recognise and abide by the legal requirements associated with these

- Users may download and print one copy of any publication from the public portal for the purpose of private study or research.

- You may not further distribute the material or use it for any profit-making activity or commercial gain

If the publication is distributed under the terms of Article $25 \mathrm{fa}$ of the Dutch Copyright Act, indicated by the "Taverne" license above, 


\section{Mechanisms of Action of Atrial-Specific Anti-Arrhythmic Drugs}

Vladimír Sobota

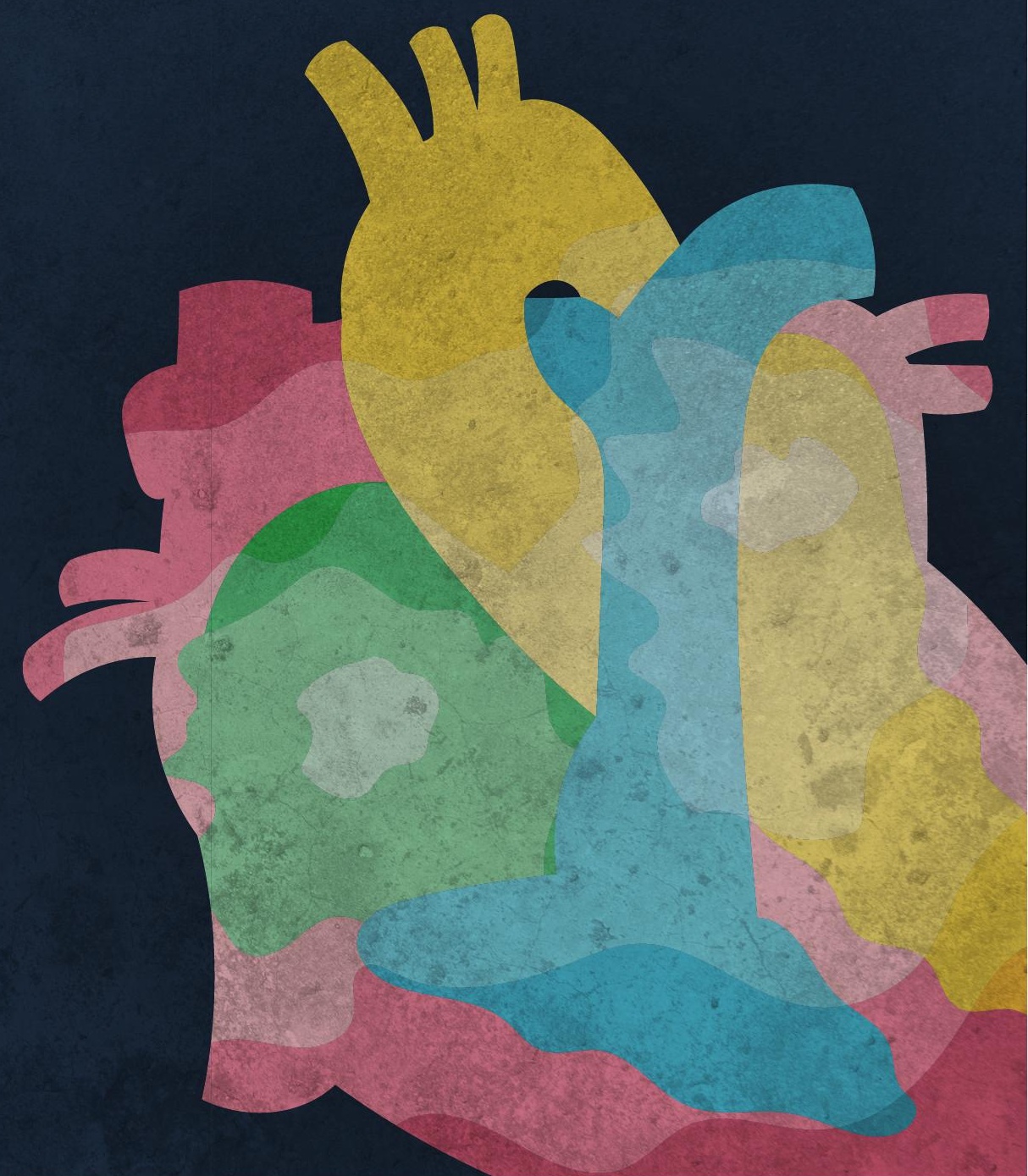





\title{
Mechanisms of Action of Atrial-Specific Anti-Arrhythmic Drugs
}

\author{
Vladimír Sobota
}




\section{COLOPHON}

Cover design: James Jardine | www.jamesjardine.nl

Layout:

James Jardine | www.jamesjardine.nl

Print:

Ridderprint | www.ridderprint.nl

ISBN:

978-94-6416-484-8

Copyright (C) 2021 by Vladimír Sobota. All rights reserved. No part of this publication may be reproduced, stored in a retrieval system or transmitted, in any form or by any means, electronic, mechanical, photocopying, recording or otherwise, without prior permission of the author or the copyright-owning journals for previous published chapters. 


\title{
Mechanisms of Action of Atrial-Specific Anti-Arrhythmic Drugs
}

\author{
Dissertation \\ to obtain the degree of Doctor at Maastricht University, \\ on the authority of the Rector Magnificus, \\ Prof. dr. Rianne M. Letschert \\ in accordance with the decision of the Board of Deans, \\ to be defended in public \\ on Friday the $28^{\text {th }}$ of May 2021 at 10.00 hours
}

by

Vladimír Sobota

Born on the $1^{\text {st }}$ of June 1990 in Vrchlabí, Czech Republic 


\section{Supervisors}

Prof. dr. U. Schotten

Dr. S. Verheule

\section{Co-supervisor}

Dr. A. van Hunnik

\section{Assessment Committee}

Prof. dr. F.W. Prinzen (chair)

Dr. W.M. Blankesteijn

Dr. R. Coronel (AMC Amsterdam)

Prof. dr. M.A. Vos (UMC Utrecht)

This research was supported by the European Union's Horizon 2020 research and innovation programme under the Marie Sklodowska-Curie grant agreement No. 675351 (AFib-TrainNet) and from the Dutch Heart Foundation; RACE V: Reappraisal of Atrial Fibrillation: Interaction between hyperCoagulability, Electrical remodeling, and Vascular Destabilization in the Progression of Atrial Fibrillation (CVON2014-09). 



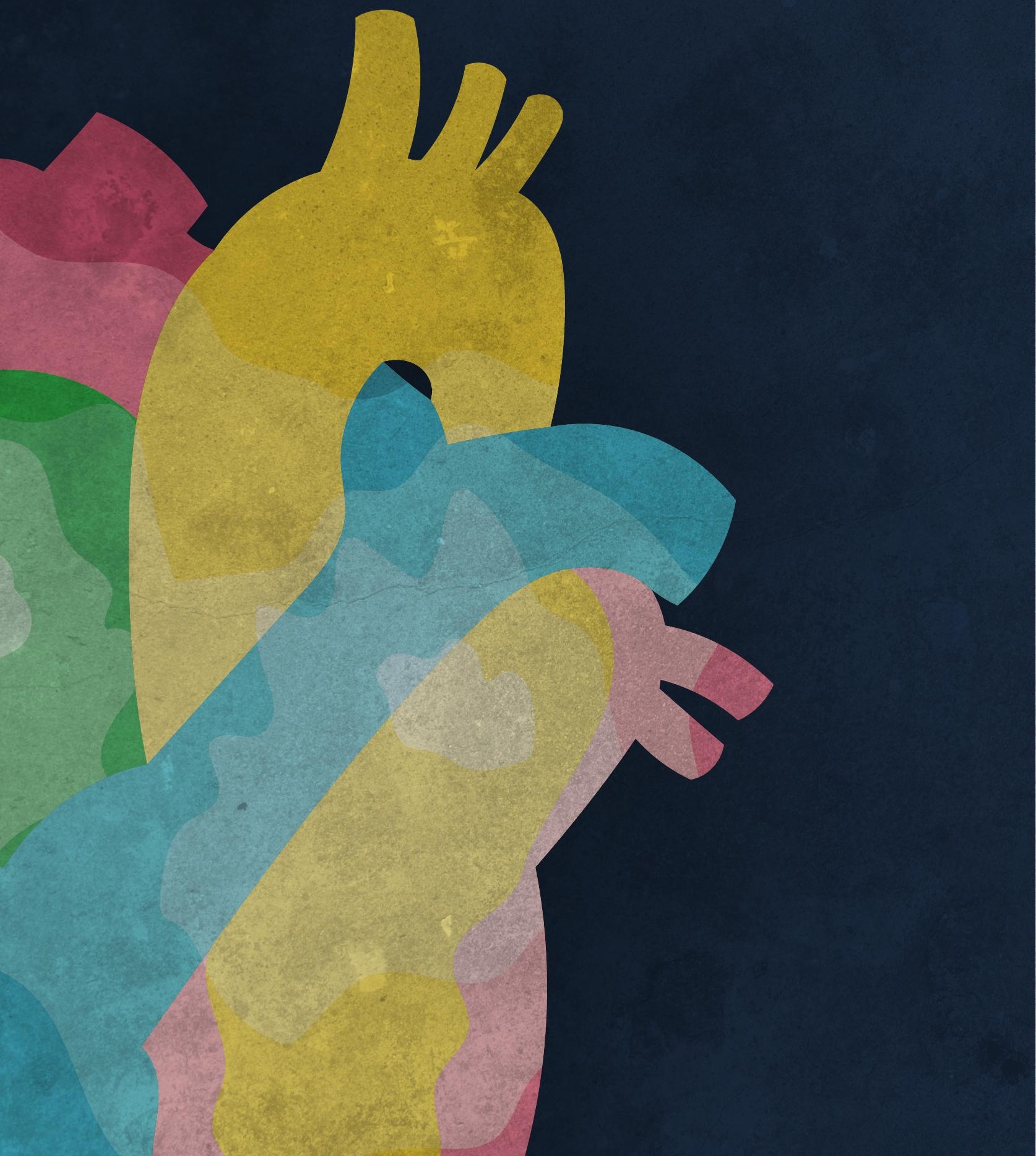




\section{CONTENTS}

Chapter 1 General introduction

Chapter 2 The acetylcholine-activated potassium current inhibitor XAF-1407 terminates persistent atrial fibrillation in goats

Chapter 3 Effective termination of atrial fibrillation by SK channel inhibition is associated with a gradual cycle length increase in contrast to a sudden organization of fibrillatory conduction

Chapter 4 Shared mechanisms of cardioversion of atrial fibrillation in the goat: acceleration of atrial conduction and inter-atrial synchronization

Chapter 5 A novel method for detection of atrial activations in optical mapping recordings of atrial fibrillation

Chapter 6 Atrial repolarization pattern is more similar to the preceding activation pattern during atrial fibrillation than during atrial pacing

Chapter 7 General discussion

Appendix Summary

Impact paragraph

Acknowledgments

About the author 


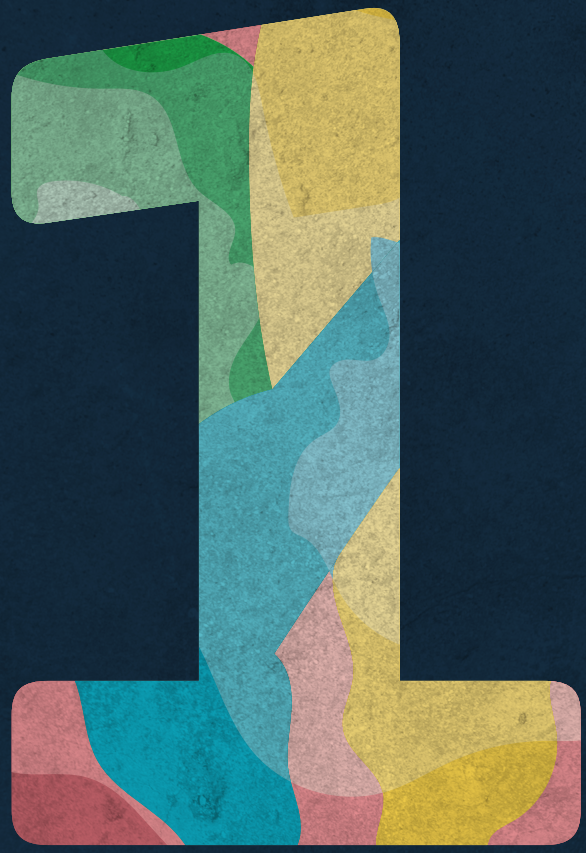


General introduction 

Atrial fibrillation (AF) is the most common sustained arrhythmia in the world. It affects approximately $3 \%$ of adults aged 20 years and older. ${ }^{1}$ The lifetime risk for developing AF in Europe and USA is up to $25 \%^{2,3}$ and it has been estimated that by 2030 there will be up to 13 million patients with AF in EU. ${ }^{4,5}$ Patients suffering from AF may experience palpitations, fatigue, shortness of breath, depressed mood, ${ }^{6}$ cognitive impairment ${ }^{7}$ and overall decreased quality of life. ${ }^{8}$ Each year, $18-30 \%$ of patients that have been diagnosed with AF are hospitalized. ${ }^{9,10}$ AF represents a major cause of stroke, heart failure and overall cardiovascular morbidity. ${ }^{11-13}$ The risk of all-cause mortality in patients with AF is increased two-fold in women and 1.5 -fold in men. ${ }^{12,14,15}$

Most AF episodes are asymptomatic and terminate spontaneously. ${ }^{16}$ However, the progressive behavior of the disease causes that over time the episodes prolong until AF becomes persistent. ${ }^{12,17-19}$ This process, known as 'AF begets $\mathrm{AF}^{20},{ }^{20}$ is characterized by proarrhythmic remodeling of atria at various physiological levels. The fast atrial rates during AF cause shortening of atrial refractoriness and loss of rate adaptation of the atrial effective refractory period (aERP). ${ }^{21,22}$ Similarly, shortening of atrial action potential duration (APD) has been observed in AF patients. ${ }^{20-22}$ This process of adaptation of atrial tissue within the first days after AF initiation is known as electrical remodeling and is associated with changes in ion channel expression and regulation. ${ }^{18,20,23}$ The density of the L-type calcium channels decreases ${ }^{24}$ and the transient outward potassium current $\left(\mathrm{I}_{\mathrm{to}}\right)$ is reduced. ${ }^{24,25}$ By contrast, the inward rectifier potassium current $\left(\mathrm{I}_{\mathrm{K} 1}\right)^{24,25}$ and constitutively active acetylcholine-activated potassium current $\left(\mathrm{I}_{\mathrm{KACh}}\right)^{26}$ were found to be increased in patients with a history of AF. All these changes can contribute to APD shortening, as schematically illustrated in Figure 1. Electrical remodeling is a reversible process, because these electrophysiological alterations can disappear already within a couple of days once the sinus rhythm is restored. ${ }^{27,28}$

In addition to electrical remodeling, $\mathrm{AF}$ is also associated with structural remodeling of atria. This process develops more slowly, over a time course of months, and is not fully reversible. ${ }^{29,30}$ The most important structural alterations recognized under this term include increased atrial fibrosis, ${ }^{31,32}$ atrial dilatation ${ }^{33}$ and myocyte hypertrophy. ${ }^{34}$ Atrial fibrosis is thought to be one of the most important factors of structural remodeling, playing a pivotal role in the formation of the AF substrate. ${ }^{35}$ Atrial fibrosis itself might be sufficient to increase AF vulnerability. ${ }^{32}$ In patients with chronic AF atrial fibrosis is more pronounced than in patients with sinus rhythm. ${ }^{36,37}$ It has been also reported that the degree of fibrosis positively correlates with postoperative $\mathrm{AF}^{38}$ and $\mathrm{AF}$ recurrence. ${ }^{36}$ Animal experiments have shown that atrial fibrosis is associated with local conduction heterogeneities and electrical dissociation between muscle bundles, ${ }^{39}$ causing disturbances in propagation of depolarization wavefronts. ${ }^{40,41}$ As a 


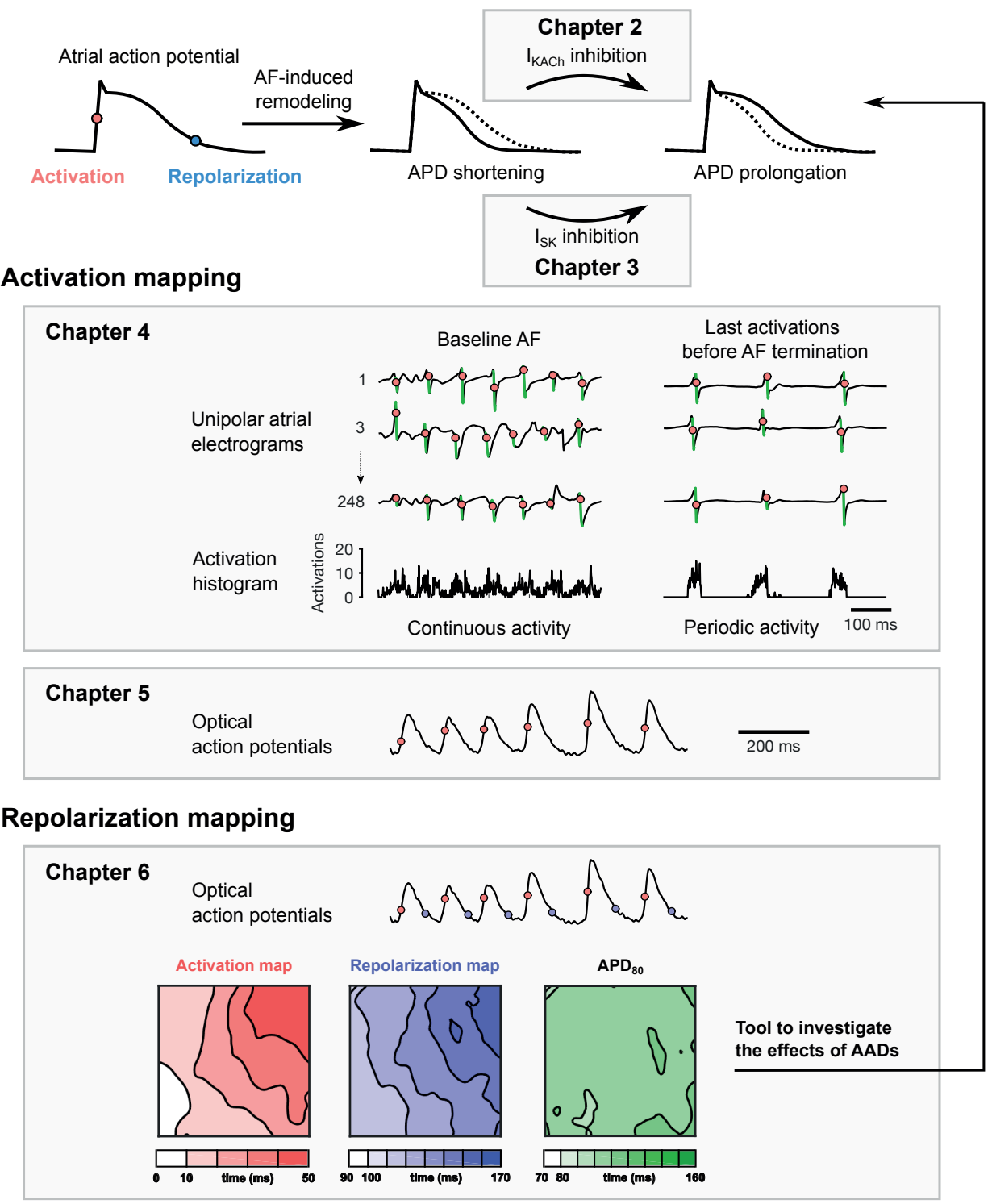

Figure 1. Schematic outline of the thesis. AF-induced remodeling is associated with shortening of atrial action potential duration (APD). The antiarrhythmic action of the studied compounds XAF-1407 and AP14145 was investigated in chapter 2 and chapter 3, respectively. In chapter 4 we further studied the process of AF cardioversion by using high-density electrical mapping data acquired in the last seconds preceding AF termination. Chapter 5 describes a novel method for detection of atrial activations in optical mapping recordings of AF. This method is further developed and used in chapter $\mathbf{6}$ that investigates the activation and repolarization patterns in perfused hearts of goats with a history of pacing-induced AF. This technique has a potential to become a useful tool for exploring antiarrhythmic as well as proarrhythmic effects of antiarrhythmic drugs (AADs). 
consequence of these changes, fibrillatory conduction becomes more complex and AF more stable. ${ }^{42-44}$ However, it should be noted that structural changes are not related exclusively to AF, but also to the degree of underlying structural heart disease present in an individual patient. ${ }^{18}$

Clinical management of AF consists of three main strategies: anticoagulation therapy, symptom control by either rhythm or rate control, and optimal treatment of the underlying cardiovascular diseases. As patients with AF have a significantly increased risk of stroke, ${ }^{12,13}$ anticoagulation therapy represents an essential part of the AF treatment. ${ }^{11}$ Fast and irregular ventricular rates caused by AF are responsible for most of the AF-related symptoms $s^{45}$ and when untreated, may even lead to tachycardiomyopathy. ${ }^{46}$ The main strategies for management of ventricular rate are rhythm control and rate control. Rate control aims to reduce the ventricular rate either by using agents blocking atrioventricular nodal conduction or - in case of severe symptoms and if pharmacological rate control is insufficient - by ablation of the atrioventricular junction. ${ }^{45}$ On the contrary, the rhythm control strategy is based on the restoration of sinus rhythm by actively terminating AF. It can be achieved by antiarrhythmic drugs, electrical cardioversion or catheter ablation. ${ }^{11,47}$

The efficacy of rhythm control therapy with antiarrhythmic drugs markedly declines with progression of the arrhythmia. ${ }^{48}$ Moreover, the use of antiarrhythmic drugs is often accompanied with significant adverse effects, ${ }^{49}$ since most of the drugs affect also ventricular electrophysiology. For example, the sodium current inhibitors may increase mortality in patients with ischemic heart disease. ${ }^{50}$ Ventricular proarrhythmicity was also reported for potassium current inhibitors. ${ }^{51}$ For this reason and because excess mortality of patients with AF primarily occurs within the first year of AF, early initiation of rhythm control therapy with antiarrhythmic drugs has been proposed..$^{52}$ This approach was associated with lower risk of cardiovascular-related outcomes when compared to usual therapy. ${ }^{53}$ However, the number of AF patients experiencing serious adverse effects due to the use of antiarrhythmic drugs for rhythm control therapy was still considerably high, reaching almost 5\%. ${ }^{53}$ Development of safer, atrial-specific antiarrhythmic drugs is therefore highly desirable to allow efficient rhythm control management of AF with less adverse effects.

\section{New targets for treatment of AF}

The acetylcholine-activated potassium current $\left(\mathrm{I}_{\mathrm{KACh}}\right)$ has been proposed as an interesting target for treatment of AF. It is an inward rectifying current that contributes to stabilization of the resting membrane potential and phase 3 repolarization. ${ }^{54} \mathrm{I}_{\mathrm{KACh}}$ is predominantly present in atria, ${ }^{55}$ sinoatrial node ${ }^{56}$ and atrioventricular node. ${ }^{57}$ Constitutively active $\mathrm{I}_{\mathrm{KACh}}$, i.e., $\mathrm{I}_{\mathrm{KACh}}$ that is active in the absence of acetylcholine, has been found in patients with chronic AF, contributing to the increased total inward rectifier potassium current in AF-remodeled atria. ${ }^{26}$ Several studies investigated $\mathrm{I}_{\mathrm{KACh}}$ inhibition as a target for treatment of AF, showing promising 
results in animal models ${ }^{58,59}$ and in isolated atrial myocytes of patients with chronic $\mathrm{AF}^{26}$ Unfortunately, the positive results obtained from preclinical studies were not successfully replicated in clinical trials. ${ }^{58,59}$ Despite that, $\mathrm{I}_{\mathrm{KACh}}$ still receives considerable attention as a target for treatment of AF, because inhibition of constitutively active $I_{\mathrm{KACh}}$ could potentially represent not only atrial-selective but also AF-selective therapy.

Recently, a new highly selective $\mathrm{I}_{\mathrm{KACh}}$ inhibitor XAF-1407 was developed, showing promising results in equine model of persistent $A F{ }^{60}$ Using a goat model of pacing-induced $A F$, in chapter 2 we investigate the cardioversion efficacy of XAF-1407 and its effects on atrial refractoriness and various electrophysiological parameters. Since $\mathrm{I}_{\mathrm{KACh}}$ is assumed to be constitutively active in electrically remodeled atria, we performed experiments in goats with and without electrical remodeling to assess the effect of $\mathrm{I}_{\mathrm{KACh}}$ inhibition in both conditions.

Another promising atrial-selective target for treatment of AF is a small conductance calciumactivated potassium (SK) channel. ${ }^{61}$ There are three subtypes of the SK channels, of which two are predominantly expressed in atria. Being dependent on the intracellular calcium concentration, SK channels affect the plateau of the action potential and terminal repolarization. ${ }^{62}$ Evidence from human cardiomyocytes showed that SK channels may also affect depolarization of the resting membrane potential and the steepness of the AP upstroke. ${ }^{62,63}$ Inhibition of SK channels leads to prolongation of APD and aERP and causes shortening of AF paroxysms. ${ }^{62,64,65} \mathrm{~A}$ recently developed SK channel inhibitor AP14145 showed selective SK channel inhibiting properties in human embryonic kidney cells. ${ }^{66,67}$ In a pig model of AF, it showed higher cardioversion efficacy than the clinically available drug vernakalant. ${ }^{67}$ Chapter 3 studies the antiarrhythmic mechanisms of AP14145 during AF and regular rhythms in a goat model of persistent AF. To obtain insights into its effects on atrial conduction and AF termination, we performed bi-atrial high-density electrical mapping. Particular attention was paid to the potential class I effect of the SK channel inhibition by AP14145.

Chapters 2 and 3 provide characterizations of two novel antiarrhythmic drugs, XAF-1407 and AP14145. To describe their antiarrhythmic effects, standard electrophysiological parameters were used, including aERP, AF cycle length (AFCL) and conduction velocity. Although providing insights into the effects these agents have on electrophysiological properties, these parameters cannot fully explain the antiarrhythmic mechanisms of these drugs. Therefore, to describe how XAF-1407 and AP14145 affect fibrillatory conduction and what their mechanism to convert $\mathrm{AF}$ to sinus rhythm is, we have performed bi-atrial high-density electrical mapping of atria. 


\section{Electrical mapping}

Mapping methods can characterize the spread of excitation in atrial tissue and thus can substantially contribute to our understanding of the mechanisms that are responsible for normal rhythm and perpetuation of arrhythmias. Mapping of cardiac activation based on unipolar or bipolar electrograms has been fundamental for identification of some of the arrhythmogenic mechanisms, including anatomical and functional reentry, ${ }^{68,69}$ endo-epicardial dissociation ${ }^{70}$ or epicardial breakthrough. ${ }^{71}$ Investigating how antiarrhythmic drugs affect the propagation patterns during $\mathrm{AF}$ allows to elucidate the mechanisms of $\mathrm{AF}$ perpetuation as well as the antiarrhythmic mechanisms of these drugs.

Using electrical mapping, several studies have described the mechanisms leading to AF termination. ${ }^{72,73}$ Some of these studies were performed in the goat model of pacing-induced $\mathrm{AF}$ - the same model we have used in chapters 2 and 3. ${ }^{72,74}$ In chapter $\mathbf{4}$ we used the mapping data from these two chapters and combined them with mapping data from other studies that investigated the effect of antiarrhythmic drugs in the same goat model. One study investigated vernakalant, ${ }^{75,76}$ a new clinically used antiarrhythmic drug that inhibits multiple ion currents including the ultra-rapid delayed rectifier current $\left(\mathrm{I}_{\text {Kur }}\right)$, delayed rectifier potassium current $\left(\mathrm{I}_{\mathrm{Kr}}\right), \mathrm{I}_{\mathrm{KACh}}, \mathrm{I}_{\text {to }}$ and sodium current. ${ }^{77}$ The second study investigated the antiarrhythmic effects of pentamidine-analogue 6 , a highly selective inhibitor of $\mathrm{I}_{\mathrm{K} 1}{ }^{78}$ To investigate the mechanisms underlying AF termination in presence of these drugs, we used unipolar atrial electrograms capturing the last 10 seconds before cardioversion and performed a systematic analysis, focusing on the beat-to-beat changes in electrophysiological properties in the last beats preceding AF termination.

Although electrical contact mapping is able to provide essential knowledge about the mechanisms perpetuating cardiac arrhythmias, it cannot be used to study all related phenomena. One of the main disadvantages of electrical contact mapping, especially when used for mapping atria, is the difficulty to reliably identify repolarization times. ${ }^{79,80}$ Although assessment of atrial repolarization from bipolar electrograms has been demonstrated as technically feasible ${ }^{81}$ such approach still requires further validation. ${ }^{82}$ An alternative approach for mapping cardiac tissue that allows analysis of cardiac repolarization is offered by optical mapping. ${ }^{83}$

\section{Optical mapping}

The possibility to record transmembrane potentials by using optical mapping with voltagesensitive fluorescence dyes was first demonstrated in neurons in $1960 \mathrm{~s} .{ }^{84,85}$ One decade later, Salama and Morad demonstrated feasibility of this method in cardiac preparations, showing that optical mapping has a potential to capture the propagation of tissue excitation across the heart. ${ }^{86}$ The technique is based on the use of potentiometric dyes that change their photon 
absorbance and emittance in response to the changes in electric field. ${ }^{83}$ The dyes bind cellular membranes and their spectral properties change in response to the changes in membrane voltage..$^{83}$

Optical mapping acquisition systems record optical action potentials with high spatial and temporal resolution, allowing assessment of repolarization and thus calculation of APD. ${ }^{87}$ Moreover, based on the physical principle used, the recorded signals are not affected by the artefacts produced by electrical stimulation or defibrillation. ${ }^{88}$ Optical mapping has therefore become a fundamental technique for exploration of the mechanisms underlying cardiac arrhythmias as well as for studying cardiac pacing and defibrillation. The method has provided important insights into the mechanisms underlying AF, identifying small stable reentrant sources $^{89}$ and intramural reentry ${ }^{90}$ as possible driving mechanisms of AF.

The expansion of optical mapping led to the development of algorithms for processing of the data acquired by this technique. ${ }^{87,91,92}$ Unfortunately, most of the available software packages perform well for regular rhythms but may underperform when used for analysis of arrhythmias. Applying these methods for recording of irregular rhythms, some action potentials, especially those with aberrant morphology, may remain undetected. In addition, the assessment of atrial repolarization may not be feasible due to the absence of diastolic intervals of sufficient length that contain the level of full repolarization. Algorithms designed specifically for analysis of cardiac arrhythmias, are still relatively scarce. In chapter 5 we propose a novel algorithm for detection of atrial activations that is tailored specifically for irregular rhythms, particularly AF. This approach is based on sensitive template matching that has been successfully used for detection of activations in high-density electrical mapping of AF. ${ }^{3}$ The method is combined with spatiotemporal filtering that allows to correct for highly-sensitive detection of action potential upstrokes. The performance of the algorithm is demonstrated on optical mapping data from perfused explanted hearts of goats with a history of pacing-induced AF. ${ }^{44}$

Many studies that applied optical mapping to investigate the mechanisms underlying AF perpetuation have focused on reconstruction of activation patterns ${ }^{87,94,95}$ or on analysis of the arrhythmia in frequency ${ }^{94,96}$ or phase domain..$^{87,97,98}$ Although one of the main advantages of optical mapping is its ability to assess repolarization times, only few studies applied this technique to assess atrial repolarization, focusing mainly on spatial dispersion of APD. ${ }^{99-101}$ In chapter $\mathbf{6}$ we characterize the relationship between activation and repolarization patterns during atrial pacing and $\mathrm{AF}$ on a beat-to beat basis. To do so, we further developed the algorithm for the detection of atrial activations described in chapter 5 and applied it on optical mapping data from perfused goat hearts with a history of pacing-induced AF. 


\section{REFERENCES}

1. Bjorck S, Palaszewski B, Friberg L, Bergfeldt L. Atrial fibrillation, stroke risk, and warfarin therapy revisited: a population-based study. Stroke 2013;44:3103-3108.

2. Lloyd-Jones DM, Wang TJ, Leip EP, Larson MG, Levy D, Vasan RS, et al. Lifetime risk for development of atrial fibrillation: The Framingham Heart Study. Circulation 2004;110:1042-1046.

3. Heeringa J, van der Kuip DAM, Hofman A, Kors JA, van Herpen G, Stricker BHC, et al. Prevalence, incidence and lifetime risk of atrial fibrillation: the Rotterdam study. Eur Heart J 2006;27:949-953.

4. Krijthe BP, Kunst A, Benjamin EJ, Lip GY, Franco OH, Hofman A, et al. Projections on the number of individuals with atrial fibrillation in the European Union, from 2000 to 2060. Eur Heart J 2013;34:2746-2751.

5. Zoni-Berisso M, Lercari F, Carazza T, Domenicucci S. Epidemiology of atrial fibrillation: European perspective. Clin Epidemiol 2014;6:213-220.

6. von Eisenhart Rothe A, Hutt F, Baumert J, Breithardt G, Goette A, Kirchhof P, et al. Depressed mood amplifies heart-related symptoms in persistent and paroxysmal atrial fibrillation patients: a longitudinal analysis-data from the German Competence Network on Atrial Fibrillation. Europace 2015;17:1354-1362.

7. Knecht S, Oelschläger C, Duning T, Lohmann H, Albers J, Stehling C, et al. Atrial fibrillation in stroke-free patients is associated with memory-impairment and hippocampal atrophy. Eur Heart J 2008;29:2125-2132.

8. Thrall G, Lane D, Carroll D, Lip GY. Quality of life in patients with atrial fibrillation: a systematic review. Am J Med 2006;119:448.e1-e19.

9. Steinberg BA, Kim S, Fonarow GC, Thomas L, Ansell J, Kowey PR, et al. Drivers of hospitalization for patients with atrial fibrillation: Results from the Outcomes Registry for Better Informed Treatment of Atrial Fibrillation (ORBIT-AF). Am Heart J 2014;167:735-742.

10. Kirchhof P, Schmalowsky J, Pittrow D, Rosin L, Kirch W, Wegscheider K, et al. Management of patients with atrial fibrillation by primary-care physicians in Germany: 1-year results of the ATRIUM Registry. Clin Cardiol 2014;37:277-284.

11. Hindricks G, Potpara T, Dagres N, Arbelo E, Bax JJ, Blomström-Lundqvist C, et al. 2020 ESC Guidelines for the diagnosis and management of atrial fibrillation developed in collaboration with the European Association of Cardio-Thoracic Surgery (EACTS). Eur Heart J 2020;42:373-498.

12. Stewart S, Hart CL, Hole DJ, McMurray J. A population-based study of the long-term risks associated with atrial fibrillation: 20-year follow-up of the Renfrew/Paisley Study. Am J Med 2002;113:359-364.

13. Wolf PA, Abbott RD, Kannel WB. Atrial Fibrillation as an Independent Risk Factor for Stroke: The Framingham Study. Stroke 1991;22:983-988.

14. Benjamin EJ, Wolf PA, d'Agostino RB, Silbershatz H, Kannel WB, Levy D. Impact of Atrial Fibrillation on the Risk of Death: the Framingham Heart Study. Circulation 1998;98:946-952.

15. Andersson T, Magnuson A, Bryngelsson I, Frøbert O, Henriksson KM, Edvardsson N, et al. Allcause mortality in 272186 patients hospitalized with incident atrial fibrillation 1995-2008: a Swedish nationwide long-term case-control study. Eur Heart J 2013;34:1061-1067. 
16. Israel CW, Grönefeld G, Ehrlich JR, Li YG, Hohnloser SH. Long-term risk of recurrent atrial fibrillation as documented by an implantable monitoring device: implications for optimal patient care. J Am Coll Cardiol 2004;43:47-52.

17. Kopecky SL, Gersh BJ, McGoon MD, Whisnant JP, Holmes DR, Jr., Ilstrup DM, et al. The natural history of lone atrial fibrillation. A population-based study over three decades. N EnglJ Med 1987;317:669-674.

18. Schotten U, Verheule S, Kirchhof P, Goette A. Pathophysiological mechanisms of atrial fibrillation: a translational appraisal. Physiol Rev 2011;91:265-325.

19. Kerr CR, Humphries KH, Talajic M, Klein GJ, Connolly SJ, Green M, et al. Progression to chronic atrial fibrillation after the initial diagnosis of paroxysmal atrial fibrillation: results from the Canadian Registry of Atrial Fibrillation. Am Heart J 2005;149:489-496.

20. Wijffels MCEF, Kirchhof CJHJ, Dorland R, Allessie MA. Atrial Fibrillation Begets Atrial Fibrillation: A Study in Awake Chronically Instrumented Goats. Circulation 1995;92:1954-1968.

21. Attuel P, Childers R, Cauchemez B, Poveda J, Mugica J, Coumel P. Failure in the rate adaptation of the atrial refractory period: its relationship to vulnerability. Int J Cardiol 1982;2:179-197.

22. Franz MR, Karasik PL, Li C, Moubarak J, Chavez M. Electrical remodeling of the human atrium: similar effects in patients with chronic atrial fibrillation and atrial flutter. J Am Coll Cardiol 1997;30:1785-1792.

23. Allessie M, Ausma J, Schotten U. Electrical, contractile and structural remodeling during atrial fibrillation. Cardiovasc Res 2002;54:230-246.

24. Bosch RF, Zeng X, Grammer JB, Popovic K, Mewis C, Kühlkamp V. Ionic mechanisms of electrical remodeling in human atrial fibrillation. Cardiovasc Res 1999;44:121-131.

25. Van Wagoner DR, Pond AL, McCarthy PM, Trimmer JS, Nerbonne JM. Outward $\mathrm{K}^{+}$current densities and Kv1.5 expression are reduced in chronic human atrial fibrillation. Circ Res 1997;80:772-781.

26. Dobrev D, Friedrich A, Voigt N, Jost N, Wettwer E, Christ T, et al. The G protein-gated potassium current $I_{K, A C h}$ is constitutively active in patients with chronic atrial fibrillation. Circulation 2005;112:3697-3706.

27. Schotten U, Duytschaever M, Ausma J, Eijsbouts S, Neuberger HR, Allessie M. Electrical and contractile remodeling during the first days of atrial fibrillation go hand in hand. Circulation 2003;107:1433-1439.

28. Yu WC, Lee SH, Tai CT, Tsai CF, Hsieh MH, Chen CC, et al. Reversal of atrial electrical remodeling following cardioversion of long-standing atrial fibrillation in man. Cardiovasc Res 1999;42:470476.

29. Ausma J, Litjens N, Lenders MH, Duimel H, Mast F, Wouters L, et al. Time course of atrial fibrillation-induced cellular structural remodeling in atria of the goat. $J$ Mol Cell Cardiol 2001;33:2083-2094.

30. Ausma J, van der Velden HM, Lenders MH, van Ankeren EP, Jongsma HJ, Ramaekers FC, et al. Reverse structural and gap-junctional remodeling after prolonged atrial fibrillation in the goat. Circulation 2003;107:2051-2058. 
31. Nattel S. Defining "culprit mechanisms" in arrhythmogenic cardiac remodeling. Circ Res 2004;94:1403-1405.

32. Verheule S, Sato T, Everett T, Engle SK, Otten D, Rubart-von der Lohe M, et al. Increased vulnerability to atrial fibrillation in transgenic mice with selective atrial fibrosis caused by overexpression of TGF-beta1. Circ Res 2004;94:1458-1465.

33. Shi Y, Ducharme A, Li D, Gaspo R, Nattel S, Tardif JC. Remodeling of atrial dimensions and emptying function in canine models of atrial fibrillation. Cardiovasc Res 2001;52:217-25.

34. Ausma J, Wijffels MCEF, Thoné F, Wouters L, Allessie M, Borgers M. Structural changes of atrial myocardium due to sustained atrial fibrillation in the goat. Circulation 1997;96:3157-3163.

35. Xintarakou A, Tzeis S, Psarras S, Asvestas D, Vardas P. Atrial fibrosis as a dominant factor for the development of atrial fibrillation: facts and gaps. Europace 2020;22:342-351.

36. Saito T, Tamura K, Uchida D, Saito T, Togashi M, Nitta T, et al. Histopathological features of the resected left atrial appendage as predictors of recurrence after surgery for atrial fibrillation in valvular heart disease. Circ J 2007;71:70-78.

37. Boldt A, Wetzel U, Lauschke J, Weigl J, Gummert J, Hindricks G, et al. Fibrosis in left atrial tissue of patients with atrial fibrillation with and without underlying mitral valve disease. Heart 2004;90:400-405.

38. Goette A, Juenemann G, Peters B, Klein HU, Roessner A, Huth C, et al. Determinants and consequences of atrial fibrosis in patients undergoing open heart surgery. Cardiovasc Res 2002;54:390-396.

39. Allessie MA, de Groot NM, Houben RP, Schotten U, Boersma E, Smeets JL, et al. Electropathological substrate of long-standing persistent atrial fibrillation in patients with structural heart disease: longitudinal dissociation. Circ Arrhythm Electrophysiol 2010;3:606-615.

40. Spach MS, Heidlage JF, Dolber PC, Barr RC. Electrophysiological effects of remodeling cardiac gap junctions and cell size: experimental and model studies of normal cardiac growth. Circ Res 2000;86:302-311.

41. Spach MS, Boineau JP. Microfibrosis produces electrical load variations due to loss of side-toside cell connections: a major mechanism of structural heart disease arrhythmias. Pacing Clin Electrophysiol 1997;20:397-413.

42. Spach MS, Josephson ME. Initiating reentry: the role of nonuniform anisotropy in small circuits. J Cardiovasc Electrophysiol 1994;5:182-209.

43. Verheule S, Tuyls E, van Hunnik A, Kuiper M, Schotten U, Allessie M. Fibrillatory conduction in the atrial free walls of goats in persistent and permanent atrial fibrillation. Circ Arrhythm Electrophysiol 2010;3:590-9.

44. Verheule S, Tuyls E, Gharaviri A, Hulsmans S, van Hunnik A, Kuiper M, et al. Loss of continuity in the thin epicardial layer because of endomysial fibrosis increases the complexity of atrial fibrillatory conduction. Circ Arrhythm Electrophysiol 2013;6:202-211.

45. Wyse DG, Waldo AL, DiMarco JP, Domanski MJ, Rosenberg Y, Schron EB, et al. A comparison of rate control and rhythm control in patients with atrial fibrillation. N Engl J Med. 2002;347:18251833. 
46. Calvo N, Bisbal F, Guiu E, Ramos P, Nadal M, Tolosana JM, et al. Impact of atrial fibrillationinduced tachycardiomyopathy in patients undergoing pulmonary vein isolation. Int $J$ Cardiol. 2013;168:4093-4097.

47. Haïssaguerre M, Jaïs P, Shah DC, Takahashi A, Hocini M, Quiniou G, et al. Spontaneous initiation of atrial fibrillation by ectopic beats originating in the pulmonary veins. $N$ Engl J Med 1998;339:659-666.

48. Frick M, Frykman V, Jensen-Urstad M, Ostergren J, Rosenqvist M. Factors predicting success rate and recurrence of atrial fibrillation after first electrical cardioversion in patients with persistent atrial fibrillation. Clin Cardiol 2001;24:238-244.

49. Chinitz JS, Halperin JL, Reddy VY, Fuster V. Rate or rhythm control for atrial fibrillation: update and controversies. Am J Med 2012;125:1049-1056.

50. Echt DS, Liebson PR, Mitchell LB, Peters RW, Obias-Manno D, Barker AH, et al. Mortality and morbidity in patients receiving encainide, flecainide, or placebo. The Cardiac Arrhythmia Suppression Trial. N Engl J Med. 1991;324:781-788.

51. Waldo AL, Camm AJ, deRuyter H, Friedman PL, MacNeil DJ, Pauls JF, et al. Effect of d-sotalol on mortality in patients with left ventricular dysfunction after recent and remote myocardial infarction. The SWORD Investigators. Survival With Oral d-Sotalol. Lancet 1996;348:7-12.

52. Nattel S, Guasch E, Savelieva I, Cosio FG, Valverde I, Halperin JL, et al. Early management of atrial fibrillation to prevent cardiovascular complications. Eur Heart J. 2014;35:1448-1456.

53. Kirchhof P, Camm AJ, Goette A, Brandes A, Eckardt L, Elvan A, et al. Early Rhythm-Control Therapy in Patients with Atrial Fibrillation. N Engl J Med 2020;383:1305-1316.

54. Milnes JT, Madge DJ, Ford JW. New pharmacological approaches to atrial fibrillation. Drug Discov Today 2012;17:654-659.

55. Koumi S, Wasserstrom JA. Acetylcholine-sensitive muscarinic $\mathrm{K}^{+}$channels in mammalian ventricular myocytes. Am J Physiol 1994;266:H1812-H1821.

56. Noma A, Trautwein W. Relaxation of the ACh-Induced Potassium Current in the Rabbit Sinoatrial Node Cell. Pflügers Arch 1978;377:193-200.

57. Sakmann B, Noma A, Trautwein W. Acetylcholine activation of single muscarinic $\mathrm{K}^{+}$channels in isolated pacemaker cells of the mammalian heart. Nature 1983;303:250-253.

58. Walfridsson H, Anderson KP, Anfinsen OG, Berggren A, Frison L, Jensen S, et al. Is the acetylcholine-regulated inwardly rectifying potassium current a viable antiarrhythmic target? Translational discrepancies of AZD2927 and A7071 in dogs and humans. Europace. 2015;17:473482 .

59. Podd SJ, Freemantle N, Furniss SS, Sulke N. First clinical trial on specific $I_{\text {KACh }}$ blocker shows no reduction in atrial fibrillation burden in patients with paroxysmal atrial fibrillation: pacemaker assessment of BMS 914392 in patients with paroxysmal atrial fibrillation. Europace 2016;18:340346.

60. Fenner MF, Carstensen H, Nissen SD, Hesselkilde EZ, Lunddahl C, Jensen MA, et al. Effect of selective $\mathrm{I}_{K, A c h}$ inhibition by XAF-1407 in an equine model of tachypacing-induced persistent atrial fibrillation. Br J Pharmacol 2020;177:3778-3794. 
61. Ehrlich JR, Biliczki P, Hohnloser SH, Nattel S. Atrial-selective approaches for the treatment of atrial fibrillation. J Am Coll Cardiol 2008;51:787-792.

62. Skibsbye L, Poulet C, Diness JG, Bentzen BH, Yuan L, Kappert U, et al. Small-conductance calcium-activated potassium (SK) channels contribute to action potential repolarization in human atria. Cardiovasc Res 2014;103:156-167.

63. Skibsbye L, Bengaard AK, Uldum-Nielsen AM, Boddum K, Christ T, Jespersen T. Inhibition of small conductance calcium-activated potassium (SK) channels prevents arrhythmias in rat atria during ß-adrenergic and muscarinic receptor activation. Front Physiol 2018;9:510.

64. Qi X, Diness JG, Brundel BJJ, Zhou X, Naud P, Wu C, et al. Role of small-conductance calciumactivated potassium channels in atrial electrophysiology and fibrillation in the dog. Circulation 2014;129:430-440.

65. Skibsbye L, Diness JG, Sorensen U, Hansen RS, Grunnet M. The Duration of Pacing-Induced Atrial Fibrillation Is Reduced in Vivo by Inhibition of Small Conductance $\mathrm{Ca}^{2+}$-activated $\mathrm{K}^{+}$ Channels. J Cardiovasc Pharmacol 2011;57:672-681.

66. Simó-Vicens R, Kirchhof JE, Dolce B, Abilgaard L, Speerschneider T, Sorensen U, et al. A new negative allosteric modulator AP14145, for the study of small conductance calcium-activated potassium $\left(\mathrm{K}_{\mathrm{Ca}}\right.$ 2) channels. Br J Pharmacol 2017;174:4396-4408.

67. Diness JG, Skibsbye L, Simó-Vicens R, Santos JL, Lundegaard P, Citerni C, et al. Termination of vernakalant-resistant atrial fibrillation by inhibition of small-conductance $\mathrm{Ca}^{2+}$-activated $\mathrm{K}^{+}$ channels in pigs. Circ Arrhythm Electrophysiol 2017;10:e005125.

68. Haissaguerre M, Hocini M, Denis A, Shah AJ, Komatsu Y, Yamashita S, et al. Driver domains in persistent atrial fibrillation. Circulation 2014;130:530-538.

69. Narayan SM, Krummen DE, Shivkumar K, Clopton P, Rappel WJ, Miller JM. Treatment of atrial fibrillation by the ablation of localized sources: CONFIRM (Conventional Ablation for Atrial Fibrillation With or Without Focal Impulse and Rotor Modulation) trial. J Am Coll Cardiol. 2012;60:628-636.

70. Eckstein J, Maesen B, Linz D, Zeemering S, van Hunnik A, Verheule S, et al. Time course and mechanisms of endo-epicardial electrical dissociation during atrial fibrillation in the goat. Cardiovasc Res 2011;89:816-824.

71. de Groot NM, Houben RP, Smeets JL, Boersma E, Schotten U, Schalij MJ, et al. Electropathological substrate of longstanding persistent atrial fibrillation in patients with structural heart disease: epicardial breakthrough. Circulation 2010;122:1674-1682.

72. de Brito Santos PE, Duytschaever M, Allessie MA. Low-frequency oscillations of atrial fibrillation cycle length in goats: characterization and potentiation by class III antiarrhytmic almokalant. J Electrocardiol 2008;41:711-723.

73. Wang J, Bourne GW, Wang Z, Villemaire C, Talajic M, Nattel S. Comparative mechanisms of antiarrhythmic drug action in experimental atrial fibrillation. Importance of use-dependent effects on refractoriness. Circulation 1993;88:1030-1044.

74. Shan Z, van der Voort PH, Blaauw Y, Duytschaever M, Allessie MA. Fractionation of electrograms and linking of activation during pharmacologic cardioversion of persistent atrial fibrillation in the goat. J Cardiovasc Electrophysiol. 2004;15:572-580. 
75. van Hunnik A, Lau DH, Zeemering S, Kuiper M, Verheule S, Schotten U. Antiarrhythmic effect of vernakalant in electrically remodeled goat atria is caused by slowing of conduction and prolongation of postrepolarization refractoriness. Heart Rhythm 2016;13:964-972.

76. van Hunnik A, Nasrallah H, Lau DH, Kuiper M, Verheule S, Schotten U. Vernakalant does not alter early repolarization or contractility in normal and electrically remodelled atria. Europace 2018;20:140-148.

77. Tsuji Y, Dobrev D. Safety and efficacy of vernakalant for acute cardioversion of atrial fibrillation: an update. Vasc Health Risk Manag 2013;9:165-175.

78. Ji Y, Varkevisser R, Opacic D, Bossu A, Kuiper M, Beekman JDM, et al. The inward rectifier current inhibitor PA-6 terminates atrial fibrillation and does not cause ventricular arrhythmias in goat and dog models. Br J Pharmacol 2017; 174:2576-2590.

79. Ihara Z, van Oosterom A, Hoekema R. Atrial repolarization as observable during PQ interval. J Electrocardiol 2006;39:290-297.

80. Tang WH, Ho WH, Chen YJ. Retrieving hidden atrial repolarization waves from standard surface ECGs. Biomed Eng Online 2018;17:146.

81. Verrier RL, Fuller H, Justo F, Nearing BD, Rajamani S, Belardinelli L. Unmasking atrial repolarization to assess alternans, spatiotemporal heterogeneity, and susceptibility to atrial fibrillation. Heart Rhythm 2016;13:953-961.

82. Narayan SM, Zaman JA, Baykaner T, Franz MR. Atrial fibrillation: Can electrograms be interpreted without repolarization information? Heart Rhythm 2016;13:962-963.

83. Berenfeld O, Efimov IR. Optical Mapping. Card Electrophysiol Clin 2019;11:495-510.

84. Cohen LB, Keynes RD, Hille B. Light scattering and birefringence changes during nerve activity. Nature 1968;218:438-441.

85. Salzberg BM, Davila HV, Cohen LB. Optical recordings of impulses in individual neurons of an invertebrate central nervous system. Nature 1973;246:508-509.

86. Salama G, Morad M. Merocyanine 540 as an optical probe of transmembrane electrical activity in the heart. Science 1976;191:485-487.

87. Laughner JI, Ng FS, Sulkin MS, Arthur RM, Efimov IR. Processing and analysis of cardiac optical mapping data obtained with potentiometric dyes. Am J Physiol Heart Circ Physiol 2012;303:H753765.

88. Herron TJ, Lee P, Jalife J. Optical imaging of voltage and calcium in cardiac cells \& tissues. Circ Res 2012;110:609-623.

89. Mandapati R, Skanes A, Chen J, Berenfeld O, Jalife J. Stable Microreentrant Sourcees as a Mechanism of Atrial Fibrillation in the Isolated Sheep Heart. Circulation 2000;101:194-199.

90. Hansen BJ, Zhao J, Csepe TA, Moore BT, Li N, Jayne LA, et al. Atrial fibrillation driven by microanatomic intramural re-entry revealed by simultaneous sub-epicardial and sub-endocardial optical mapping in explanted human hearts. Eur Heart J 2015;36:2390-2401.

91. O'Shea C, Holmes AP, Yu TY, Winter J, Wells SP, Correia J, et al. ElectroMap: High-throughput open-source software for analysis and mapping of cardiac electrophysiology. Sci Rep 2019;9:1389.

92. Cathey B, Obaid S, Zolotarev AM, Pryamonosov RA, Syunyaev RA, George SA, et al. OpenSource Multiparametric Optocardiography. Sci Rep. 2019;9:721. 
93. Zeemering S, Maesen B, Nijs J, Lau DH, Granier M, Verheule S, et al. Automated quantification of atrial fibrillation complexity by probabilistic electrogram analysis and fibrillation wave reconstruction. Conf Proc IEEE Eng Med Biol Soc 2012;2012:6357-6360.

94. Skanes AC, Mandapati R, Berenfeld O, Davidenko JM, Jalife J. Spatiotemporal periodicity during atrial fibrillation in the isolated sheep heart. Circulation 1998;98:1236-1248.

95. Berenfeld O, Zaitsev AV, Mironov SF, Pertsov AM, Jalife J. Frequency-dependent breakdown of wave propagation into fibrillatory conduction across the pectinate muscle network in the isolated sheep right atrium. Circ Res 2002;90:1173-1180.

96. Zaitsev AV, Berenfeld O, Mironov SF, Jalife J, Pertsov AM. Distribution of excitation frequencies on the epicardial and endocardial surfaces of fibrillating ventricular wall of the sheep heart. Circ Res 2000;86:408-417.

97. Chen J, Mandapati R, Berenfeld O, Skanes AC, Gray RA, Jalife J. Dynamics of wavelets and their role in atrial fibrillation in the isolated sheep heart. Cardiovasc Res 2000;48:220-232.

98. Filgueiras-Rama D, Price NF, Martins RP, Yamazaki M, Avula UM, Kaur K, et al. Long-term frequency gradients during persistent atrial fibrillation in sheep are associated with stable sources in the left atrium. Circ Arrhythm Electrophysiol 2012;5:1160-1167.

99. Nygren A, Lomax AE, Giles WR. Heterogeneity of action potential durations in isolated mouse left and right atria recorded using voltage-sensitive dye mapping. Am J Physiol Heart Circ Physiol 2004;287:H2634-H2643.

100. Verheule S, Wilson E, Banthia S, Everett TH, Shanbhag S, Sih HJ, et al. Direction-dependent conduction abnormalities in a canine model of atrial fibrillation due to chronic atrial dilatation. Am J Physiol Heart Circ Physiol 2004;287:H634-H644.

101. Li N, Csepe TA, Hansen BJ, Sul LV, Kalyanasundaram A, Zakharkin S, et al. Adenosine-induced atrial fibrillation: localized reentrant drivers in lateral right atria due to heterogeneous expression of adenosine A1 receptors and GIRK4 subunits in the human heart. Circulation 2016;134:486-498. 


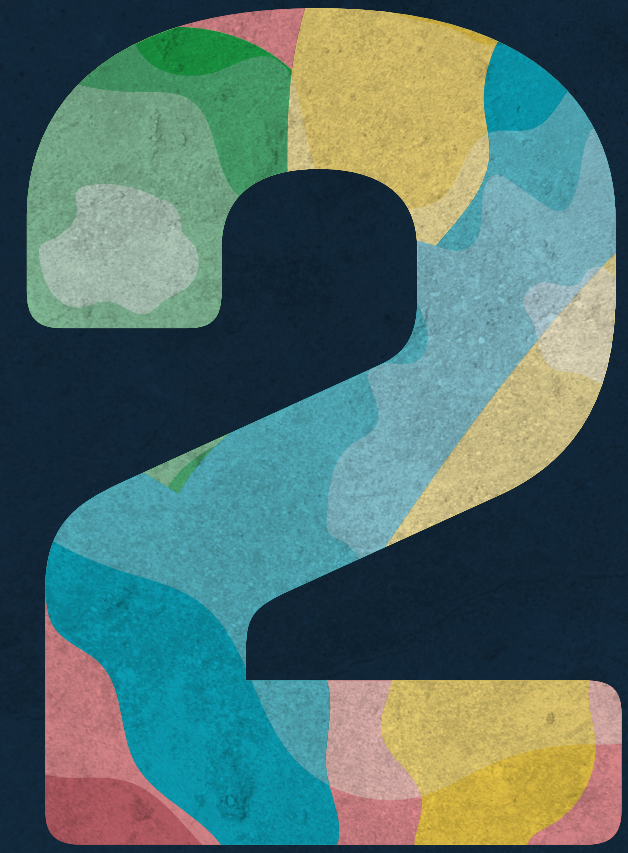




\title{
The acetylcholine-activated potassium current inhibitor XAF-1407 terminates persistent atrial fibrillation in goats
}

\author{
Vladimír Sobota, Giulia Gatta, Arne van Hunnik, Iris van Tuijn, Marion Kuiper, \\ James Milnes, Thomas Jespersen, Ulrich Schotten and Sander Verheule
}

Frontiers in Pharmacology 11:608410 


\section{ABSTRACT}

Aims: The acetylcholine-activated inward rectifier potassium current $\left(\mathrm{I}_{\mathrm{KACh}}\right)$ has been proposed as an atrial-selective target for the treatment of atrial fibrillation (AF). Using a novel selective $\mathrm{I}_{\mathrm{KACh}}$ inhibitor XAF-1407, the study investigates the effect of $\mathrm{I}_{\mathrm{KACh}}$ inhibition in goats with pacing-induced, short-term AF.

Methods: Ten goats $(57 \pm 5 \mathrm{~kg})$ were instrumented with pericardial electrodes. Electrophysiological parameters were assessed at baseline and during intravenous infusion of XAF-1407 $(0.3,3.0 \mathrm{mg} / \mathrm{kg})$ in conscious animals before and after 2 days of electrically induced AF. Following a further 2 weeks of sustained AF, cardioversion was attempted with either XAF-1407 (0.3 followed by $3 \mathrm{mg} / \mathrm{kg}$ ) or with vernakalant ( 3.7 followed by $4.5 \mathrm{mg} / \mathrm{kg}$ ), an antiarrhythmic drug that inhibits the fast sodium current and several potassium currents. During a final open chest experiment, 249 unipolar electrograms were recorded on each atrium to construct activation patterns and AF cardioversion was attempted with XAF-1407.

Results: XAF-1407 prolonged atrial effective refractory period by $36 \mathrm{~ms}$ (45\%) and $71 \mathrm{~ms}$ (87\%) $(0.3$ and $3.0 \mathrm{mg} / \mathrm{kg}$, respectively; pacing cycle length $400 \mathrm{~ms}, 2$ days of AF-induced remodeling) and showed higher cardioversion efficacy than vernakalant (8/9 vs. 5/9). XAF1407 caused a minor decrease in the number of waves per AF cycle in the last seconds prior to cardioversion. Administration of XAF-1407 was associated with a modest increase in QTc $(<10 \%)$. No ventricular proarrhythmic events were observed.

Conclusion: XAF-1407 showed an antiarrhythmic effect in a goat model of AF. The study indicates that $\mathrm{I}_{\mathrm{KACh}}$ represents an interesting therapeutic target for treatment of AF. To assess the efficacy of XAF-1407 in later time points of AF-induced remodeling, follow-up studies with longer period of AF maintenance would be necessary. 


\section{INTRODUCTION}

Atrial fibrillation (AF) is the most common clinically diagnosed cardiac arrhythmia, affecting approximately $3 \%$ of the population aged 20 years and older. ${ }^{1}$ It is associated with increased risk for stroke and death. ${ }^{1}$ AF causes both electrical and structural remodeling of the atria. ${ }^{2}$ The rapid process of electrical remodeling (hours to days) entails shortening of the atrial effective refractory period (aERP), whereas the much slower process of structural remodeling (weeks to years) refers to alterations in atrial tissue structure. Both processes contribute to the gradual progression of $\mathrm{AF}$, to increasing $\mathrm{AF}$ episode duration and to decreasing the efficacy of antiarrhythmic drugs. Furthermore, antiarrhythmic drug treatment is hampered by significant adverse effects, including ventricular proarrhythmia. Thus, there is an unmet need for development of new antiarrhythmic therapies that are atrial-specific, with fewer side effects.

The acetylcholine-activated potassium current $\left(\mathrm{I}_{\mathrm{KACh}}\right)$ is an inward rectifier that contributes to stabilization of the resting membrane potential and phase 3 repolarization. ${ }^{3} \mathrm{I}_{\mathrm{KACh}}$ is predominantly present in atria, ${ }^{4}$ sinoatrial node ${ }^{5}$ and atrioventricular (AV) node. ${ }^{6}$ Constitutively active $\mathrm{I}_{\mathrm{KACh}}$, i.e., $\mathrm{I}_{\mathrm{KACh}}$ that is active in the absence of acetylcholine, has been reported in patients with chronic $A F$, suggesting an increased contribution to the total inward rectifier $\mathrm{K}^{+}$current in atria with AF-induced remodeling. ${ }^{7}$ Selective $\mathrm{I}_{\mathrm{KACh}}$ inhibition by tertiapin reversed the shortening of action potential duration after AF-induced remodeling in patients with chronic AF, ${ }^{7}$ making $\mathrm{I}_{\mathrm{KACh}}$ an interesting atrial-selective target for treatment of AF.

Recently, a new antiarrhythmic compound XAF-1407 (3-methyl-1-[5-phenyl-4-[4-(2pyrrolidin-1-ylethoxymethyl)-1-piperidyl] thieno[2,3-d]pyrimidin-6-yl]azetidin-3-ol) was developed by Xention (Xention Ltd., United Kingdom), showing high selectivity for $\mathrm{I}_{\mathrm{KACh}}{ }^{8}$ Compared to other major ion channels in the heart, the selectivity of XAF-1407 to $\mathrm{I}_{\mathrm{KACh}}$ channels was shown to be at least 1000 fold higher. ${ }^{8}$ In this study we aimed to investigate the cardioversion efficacy of XAF-1407 and its effects on atrial refractoriness and other electrophysiological parameters. We have used a goat model of pacing-induced AF that recapitulates the $\mathrm{AF}$-induced atrial remodeling also observed in patients. ${ }^{?}$ 


\section{MATERIALS AND METHODS}

\section{Animal model}

The research protocol was approved by the local ethical board for animal experimentation and is in compliance with the European directive $2010 / 63 / \mathrm{EU}$ on the protection of animals used for scientific purposes.

Ten female Dutch milk goats from a local breeder with body weights of $57 \pm 5$ (range 47-64) kg and age 24 3 (range 20-27) months were used in the study. The animals were housed individually in conventional cages of approximately $8 \mathrm{~m}^{2}$ with straw bedding and constant temperature and humidity. The animals were kept at a 12-hour light/dark cycle with a free access to food (hay and pellets) and water. Animal welfare was checked daily and body weight measured once a week. The goat model of pacing-induced AF has been chosen because it is well characterized and has proven to provide valuable insights into the mechanisms underlying AF., ${ }^{910}$ It has been previously used to study the antiarrhythmic effect of drugs for treatment of AF. ${ }^{10-13}$ Female goats have been used in order to allow comparability with previous studies that were solely based on female goats. ${ }^{10-15}$ Other reasons for the use of female goats was their tranquil nature and a very limited availability of male goats.

A left-sided thoracotomy was performed under general anesthesia that was initiated with sodium thiopental $(20 \mathrm{mg} / \mathrm{kg})$ and maintained with isoflurane $(0.5 \%)$, sufentanyl $(6 \mu \mathrm{g} / \mathrm{kg} / \mathrm{h} \mathrm{IV})$ and propofol $(5-10 \mu \mathrm{g} / \mathrm{kg} / \mathrm{h} \mathrm{IV})$. A custom-build patch of electrodes was sutured epicardially under the left atrial (LA) appendage through a small incision in pericardium. A plaque of sensing electrodes was implanted pericardially above the LA, together with the electrodes of the implanted stimulator (Medtronic Itrel ${ }^{\ominus}$, Medtronic, Minneapolis, Minnesota, USA). Furthermore, a pair of sensing electrodes was sutured on the pericardium above both the left and the right ventricular free wall. Three silver discs $(2 \mathrm{~cm}$ diameter) were implanted subcutaneously and were used as reference electrodes. The wires of all custom-build electrodes were incorporated in a single cable, tunneled subcutaneously to the neck and exteriorized. The animals were treated post-operatively with antibiotics and received analgesia with buprenofine (5-10 $\mu \mathrm{g} / \mathrm{kg}$ IM twice a day, first day after the surgery) and carprofen $(2-4 \mathrm{mg} / \mathrm{kg}$ SC, first three days after surgery). A recovery period of 2-3 weeks was allowed before the first experiment.

\section{Effects of XAF-1407 on cardiac electrophysiology in conscious animals}

The effect of XAF-1407 was tested during pacing protocols in five conscious animals in control conditions (normal atria) and after 2 days of AF (electrically remodeled atria). The drug was administered intravenously, using sodium acetate buffer $(0.1 \mathrm{~mol} / \mathrm{l})$ as a vehicle. Two doses of XAF-1407 were investigated: 0.3 and $3.0 \mathrm{mg} / \mathrm{kg}$, infused as a bolus over 20 minutes (15 and 
$135 \mu \mathrm{g} / \mathrm{kg} / \mathrm{min}$ for the doses of 0.3 and $3.0 \mathrm{mg} / \mathrm{kg}$, respectively). To maintain stable plasma level throughout the study protocol, a one-hour maintenance infusion $(5.9$ and $59 \mu \mathrm{g} / \mathrm{kg} /$ min for the doses of 0.3 and $3.0 \mathrm{mg} / \mathrm{kg}$, respectively) was started after the bolus. Multiple plasma samples were taken during the drug administration, allowing assessment of XAF1407 concentration in free plasma by liquid chromatography-mass spectrometry. The dosing regimen was designed to reach stable plasma levels of XAF-1407 during electrophysiological measurements, as shown in Figure 1. The dose of $0.3 \mathrm{mg} / \mathrm{kg}$ corresponds to the lower range of maximum effectivity while the dose of $3.0 \mathrm{mg} / \mathrm{kg}$ represents a safe and effective dose of XAF1407 in the horse. ${ }^{8}$

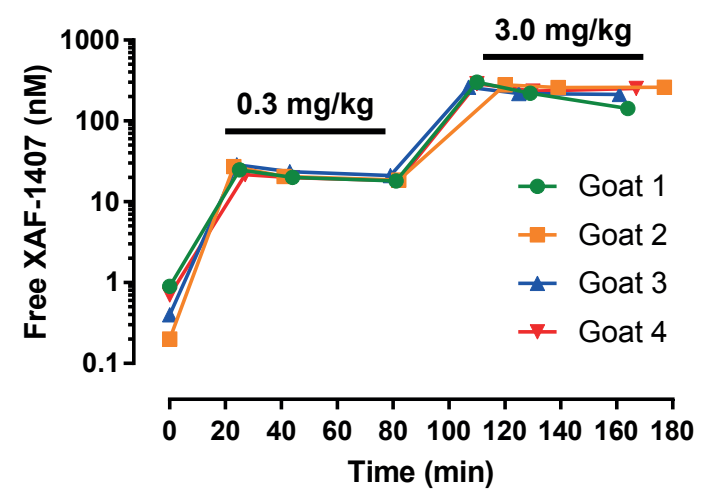

Figure 1. Free plasma concentration of XAF-1407 during the experiments in conscious goats. The data from four animals show that the dosing regimen reached stable plasma concentration of XAF-1407 in both applied doses. Time 0 corresponds with the beginning of the intravenous infusion.

Basic electrophysiological parameters were determined in normal atria and after 2 days of AF (electrically remodeled atria). AF was induced and maintained by the implanted stimulator, applying $50 \mathrm{~Hz}$ burst stimulation every other second. The aERP was determined using an S1S2 protocol. The Wenckebach cycle length (the cycle length at which a $2^{\text {nd }}$ degree AV block occurs) was determined using an S1-S1 protocol, applying cycle lengths from 500 to $200 \mathrm{~ms}$ with decremental steps of $50 \mathrm{~ms}$. Both atrial and ventricular unipolar electrograms were recorded during the measurements together with a pseudo-ECG obtained from the implanted electrodes. A period of at least 7 days followed the measurements in electrically remodeled atria to allow complete reverse remodeling of the atria. To investigate the effect of vagal activity on the $\mathrm{I}_{\mathrm{KACh}}$ inhibition by XAF-1407, atropine $(0.04 \mathrm{mg} / \mathrm{kg})$ was administered intravenously in two animals with non-remodeled atria, followed by a dose of $3.0 \mathrm{mg} / \mathrm{kg}$ of XAF-1407. 


\section{Cardioversion attempts in conscious animals}

A period of at least 7 days followed the measurements in electrically remodeled atria to allow complete reverse remodeling of the atria. ${ }^{16}$ Subsequently, AF was induced again and maintained for 3 weeks. Cardioversion attempts with XAF-1407 (dose of $0.3 \mathrm{mg} / \mathrm{kg}$ followed by $3.0 \mathrm{mg} / \mathrm{kg}$ ) were performed after 2 weeks of AF maintenance. For comparison, we used vernakalant, an antiarrhythmic drug for treatment of AF that we have recently tested in the goat model of pacing-induced AF. ${ }^{10,11}$ Vernakalant inhibits fast sodium current and several potassium currents, including the transient outward current $\left(\mathrm{I}_{\text {to }}\right)$ and has been recently approved for European market. ${ }^{17,18}$ The dosing regimen of vernakalant (dose of $3.7 \mathrm{mg} / \mathrm{kg}$ followed by $4.5 \mathrm{mg} / \mathrm{kg}$ ) was designed to reach plasma concentrations of $3 \mathrm{mg} / \mathrm{ml}$ (the dose of $3.7 \mathrm{mg} / \mathrm{kg}$ ) and $5 \mathrm{mg} / \mathrm{ml}$ (the dose of $4.5 \mathrm{mg} / \mathrm{kg}$ ), which are the plasma levels that are known to be effective in patients. ${ }^{10}$ Each dose was administered using a loading dose infused over 20 minutes, followed by a maintenance dose for another 20 minutes. After drug administration, all animals were monitored during a wash-out period of 30 minutes. The order of the cardioversion attempts was randomized, yielding the same average AF duration for each drug. One animal did not develop persistent $\mathrm{AF}$ and was excluded from the analysis of cardioversion efficacy.

\section{Open chest experiment}

A final open chest experiment was performed 23-24 days after the AF initiation. Anesthesia was induced with sodium thiopental $(20 \mathrm{mg} / \mathrm{kg})$ and maintained with sufentanyl $(6 \mu \mathrm{g} / \mathrm{kg} / \mathrm{h}$ IV), propofol (5-10 mg/kg/h IV) and rocuronium $(0.3 \mathrm{mg} / \mathrm{kg} / \mathrm{h} \mathrm{IV})$. The heart was exposed through a left-sided thoracotomy. Mapping electrode arrays (249 electrodes, interelectrode distance $2.4 \mathrm{~mm}$, array diameter $4 \mathrm{~cm}$ ) were placed on both the LA and the right atrial (RA) free wall to allow recording of unipolar atrial electrograms (sampling rate $1 \mathrm{kHz}, 16$-bit resolution, filter bandwidth 0.1-400 Hz). Standard limb leads were used to record the surface ECG. After instrumentation, a stabilization period of at least 30 minutes was allowed before the start of the measurements. Left ventricular pressures were measured using a pressure-volume catheter (Sentron Europe BV, Roden, The Netherlands). Two ten-pole catheters, positioned in the coronary sinus and along the right atrial free wall were connected to an external defibrillator (Physio-Control Lifepak 9B, Medtronic, Minneapolis, Minnesota, USA) to allow electrical cardioversion of AF.

Before the drug infusion, the heart was endocardially cardioverted by a DC shock $(\leq 20 \mathrm{~J})$ to assess aERP ( $\mathrm{S} 1-\mathrm{S} 2$ protocol; pacing output $4 \mathrm{x}$ threshold current) and conduction velocity (S1-S1 protocol; 500-200 ms, pacing output 2x threshold current). AF was reinduced by burst pacing and XAF-1407 was administered, applying an identical dosing regimen as the one that was used during electrophysiological measurements in conscious animals. Unipolar 
electrograms were recorded during the drug administration to allow later assessment of changes in AF properties. Conduction velocity (CV) and aERP were reassessed for each dose of XAF1407. Once the experiment was completed, the animal was euthanized by rapid excision of the heart.

\section{Data and Statistical Analysis}

The atrial fibrillation cycle length (AFCL) was calculated using a median window of 60 seconds. Pseudo-ECG signals were analyzed manually using the IDEEQ software (Instrument Development Engineering and Evaluation, Maastricht University, Maastricht, The Netherlands). Corrected QT intervals (QTc) were calculated by using a goat-specific version of Bazzet's formula, ${ }^{19} \mathrm{QTc}=\mathrm{QT} /(\mathrm{RR})^{0.6}$.

Custom-made software in the MATLAB programming environment (The MathWorks, Inc., Natick, Massachusetts, USA) was used for analysis of unipolar atrial electrograms. Local atrial activations were determined using a probabilistic annotation algorithm. ${ }^{20}$ The following measures of AF substrate complexity were calculated: median AF cycle length, maximum wave size, number of waves per AF cycle, number of breakthrough waves per AF cycle, fractionation index and maximum dissociation. ${ }^{20}$ For both atria, the AF properties were assessed at baseline (intervals of 60 seconds), prior to cardioversion (intervals of 1.5 to 2 seconds) and in a preceding interval of 10 seconds. Atrial wavelength was calculated as a product of aERP and CV. ${ }^{21}$

Data were statistically analyzed using IBM SPSS Statistics for Macintosh, version 25 (IBM Corp., Armonk, New York, USA) and GraphPad Prism version 7.03 (GraphPad Software, La Jolla, California, USA). A log-rank test was used to compare the cardioversion efficacy of XAF-1407 with vernakalant and the change in Wenckebach cycle length. A Friedman's test was used to compare changes in the left-ventricular hemodynamic parameters and in ECG parameters. AFCL prolongation was compared using Mann-Whitney test. A linear mixedeffects model was used for the comparison of drug-induced changes in conduction velocity. Two-way repeated measures ANOVA was used for the comparison of the changes in aERP and wavelength. The data are presented as mean \pm standard deviation unless stated otherwise. The percentual changes in ECG parameters are stated as median and interquartile range. A p-value $<0.05$ was considered as statistically significant. 
A

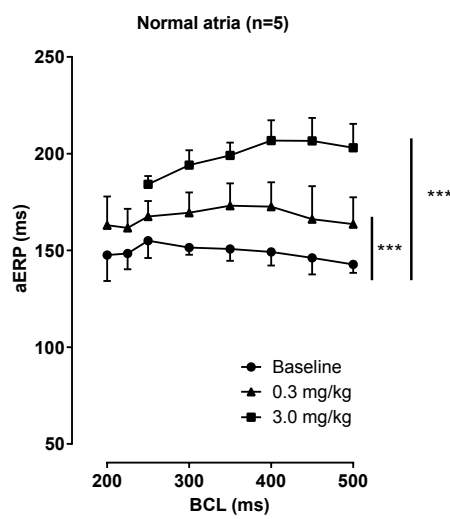

B

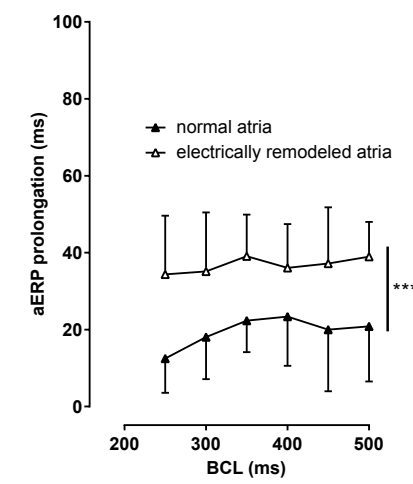

C

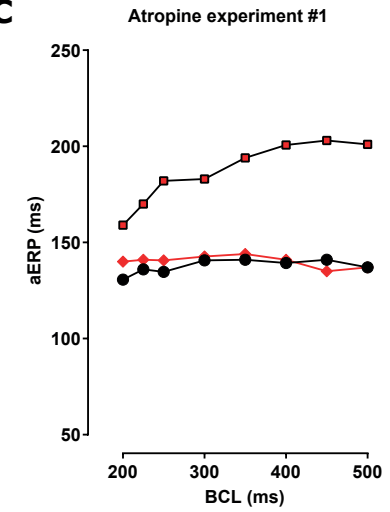

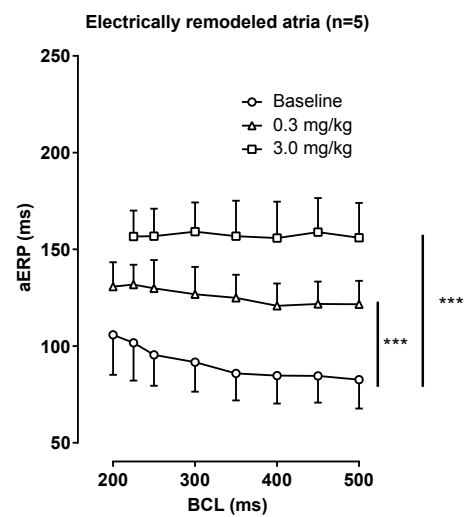
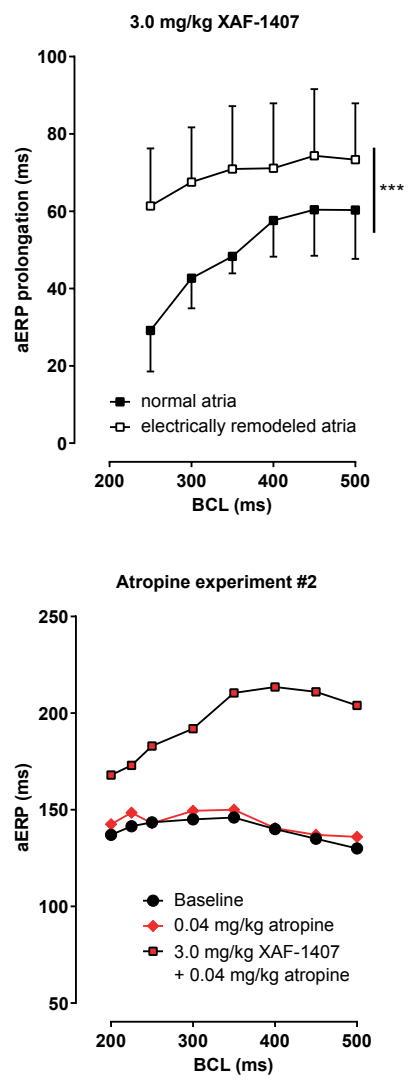

Figure 2. The effect of XAF-1407 on atrial refractoriness. (A) Prolongation of aERP at all burst cycle lengths (BCL) was observed in normal $(\mathrm{n}=5)$ and electrically remodeled atria $(\mathrm{n}=5)$ after the administration of 0.3 and $3.0 \mathrm{mg} / \mathrm{kg}$ of XAF-1407. (B) Compared to normal atria, the effect of XAF-1407 was larger after electrical remodeling for both applied doses of the drug. (C) Additional experiments with atropine showed that vagal inhibition does not alter the effect of XAF-1407 on atrial refractoriness. Mixed model, ${ }^{*} \mathrm{p}<0.05$. 


\section{RESULTS}

A dose-dependent prolongation of aERP in LA was observed after the administration of XAF1407 in normal atria as well as in electrically remodeled atria at all pacing rates (Figure 2A). The aERP prolongation caused by XAF-1407 was generally smaller at shorter cycle lengths (reverse rate dependence). The effect of XAF-1407 was larger in electrically remodeled atria than in normal atria (Figure $2 \mathrm{~B}$ ). To investigate how $\mathrm{I}_{\mathrm{KACh}}$ inhibition is affected by vagal tone, we performed aERP measurements after vagal inhibition by atropine in two normal goats without electrical remodeling. The measurements showed that atropine does not alter the efficacy of XAF-1407 to prolong aERP, as shown in Figure 2C.

A prolongation of RR interval was observed after the administration of XAF-1407 (Figure 3A). In the animals without electrical remodeling, a prolongation of RR interval by $25 \%$ (18-30\%) was observed after the administration of $0.3 \mathrm{mg} / \mathrm{kg}$ of XAF-1407 (Figure 3A). Contrary to that, the effect of the same dose after 2 days of pacing-induced AF was only limited, with a prolongation of $4 \%$ (3-11\%). The dose of $3.0 \mathrm{mg} / \mathrm{kg}$ of XAF-1407 resulted in a prolongation of RR interval by $12 \%(2-13 \%)$ and $14 \%(3-18 \%)$ in the hearts with and without pacinginduced AF, respectively. Similarly, a small prolongation of PQ by $10 \%$ (7-11\%) in normal atria and by $4 \%(3-18 \%)$ in electrically remodeled atria was observed after the administration of $3.0 \mathrm{mg} / \mathrm{kg}$ of XAF-1407 (Figure 3C). The effect of XAF-1407 on ventricular electrophysiology was limited, with the QRS prolongation in normal atria by 7\% (6-11\%) and in electrically remodeled atria by $8 \%(7-11 \%)$ after $3.0 \mathrm{mg} / \mathrm{kg}$ of XAF-1407 (Figure 3D). The same dose of XAF-1407 caused the prolongation of QTc by $8 \%(5-13 \%)$ and $9 \%(6-11 \%)$ in normal and electrically remodeled atria, respectively. (Figure 3B). No ventricular proarrhythmic events (extrasystoles, non-sustained ventricular tachycardia) were observed during administration of either vernakalant or XAF-1407. To further explore the effect of $I_{\text {KACh }}$ inhibition on the AV node, the Wenckebach cycle length was investigated in awake animals during atrial pacing. The median Wenckebach cycle length was $200 \mathrm{~ms}$ in the animals without any history of AF and $250 \mathrm{~ms}$ after atrial electrical remodeling. At $3.0 \mathrm{mg}$ of XAF-1407 the Wenckebach cycle length prolonged to 300 and $350 \mathrm{~ms}$, respectively (Figure 3E-G).

Pharmacological cardioversion of AF with either XAF-1407 or vernakalant was attempted in awake animals after $16 \pm 2$ days of AF maintenance. One animal did not develop stable AF and was excluded from the analysis of cardioversion efficacy. Infusion of XAF-1407 resulted in cardioversion in 8 out of 9 goats ( 5 cardioversions with the dose $0.3 \mathrm{mg} / \mathrm{kg}, 2$ with the dose of $3.0 \mathrm{mg} / \mathrm{kg}, 1$ during washout) with an average time to cardioversion of $40 \pm 26 \mathrm{~min}$ (Figure 4F). Compared to that, 5 out of 9 goats cardioverted during the infusion of vernakalant (0 with the dose of $3.7 \mathrm{mg} / \mathrm{kg}, 4$ cardioversions with the dose of $4.5 \mathrm{mg} / \mathrm{kg}, 1$ during washout) with an average time to cardioversion of $67 \pm 14 \mathrm{~min}$. Administration of both XAF-1407 
A

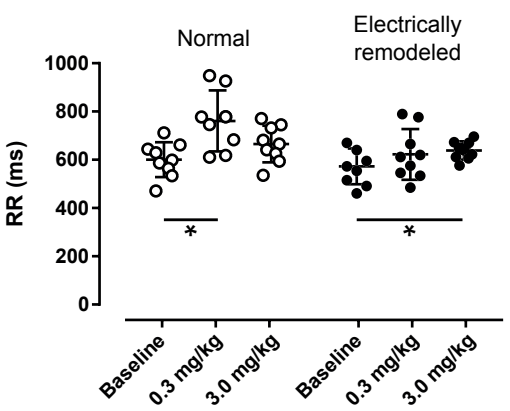

C

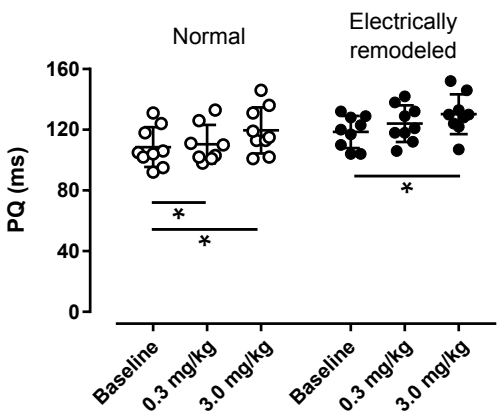

E

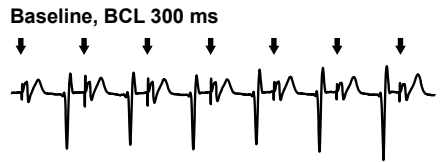

$\mathbf{F}$

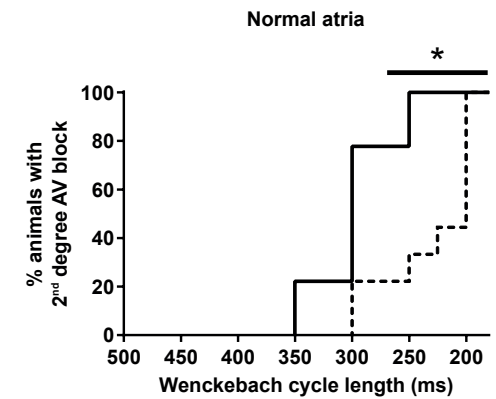

B

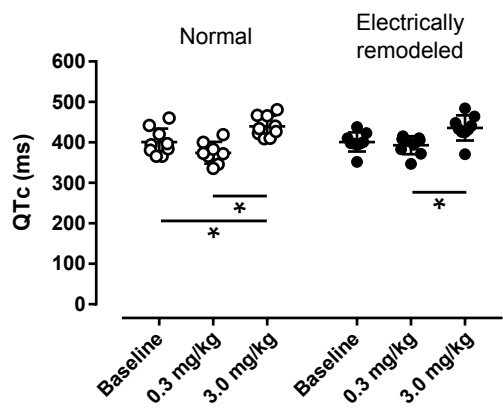

D

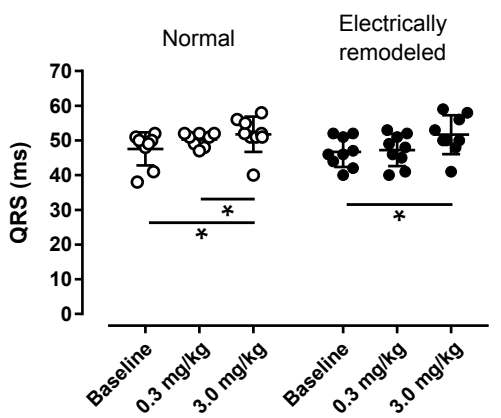

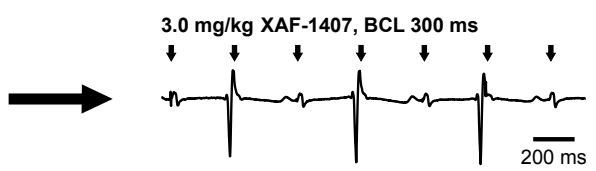

G

Electrically remodeled atria

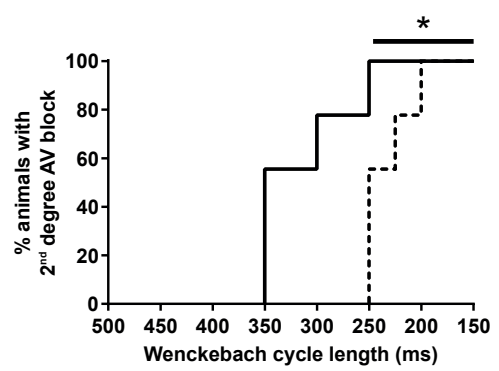

-.. Baseline $\quad-3.0 \mathrm{mg} / \mathrm{kg}$

Figure 3. Changes in electrophysiological parameters during administration of XAF-1407 in conscious animals. Prolongation of (A) RR, (B) QTc, (C) PQ and (D) QRS intervals was observed in goats with normal atria and electrically remodeled atria $(n=9)$. Statistical comparisons were made between baseline and the doses of 0.3 and $3.0 \mathrm{mg} / \mathrm{kg}$ of XAF-1407. Friedman's test, ${ }^{*} \mathrm{p}<0.05$. (E) Examples of electrocardiograms demonstrating LA pacing (BCL $300 \mathrm{~ms}$ ) during baseline and after the administration of $3.0 \mathrm{mg} / \mathrm{kg}$ of XAF-1407 illustrate the presence of $2^{\text {nd }}$ degree AV block after the drug administration. (F) Prolongation of Wenckebach cycle length was observed after the administration of $3.0 \mathrm{mg} / \mathrm{kg}$ of XAF-1407 in normal atria as well as in electrically remodeled atria (G). Log-rank test, ${ }^{*} \mathrm{p}<0.05, \mathrm{n}=9$. 
A

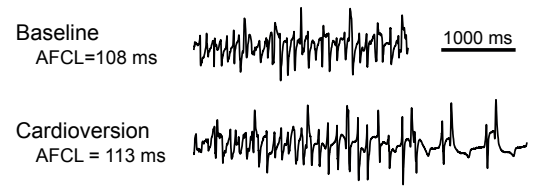

C Start of the drug

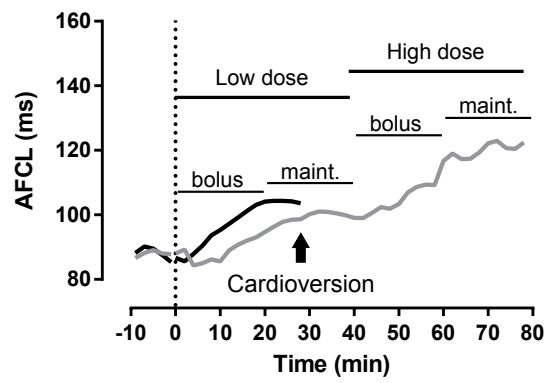

$\mathbf{E}$

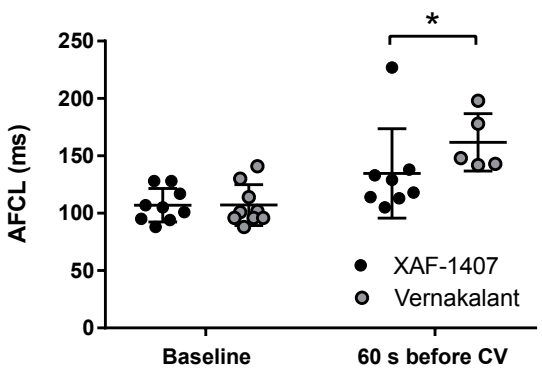

B

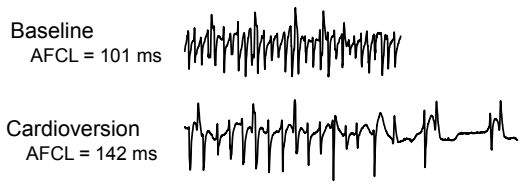

D Start of the drug

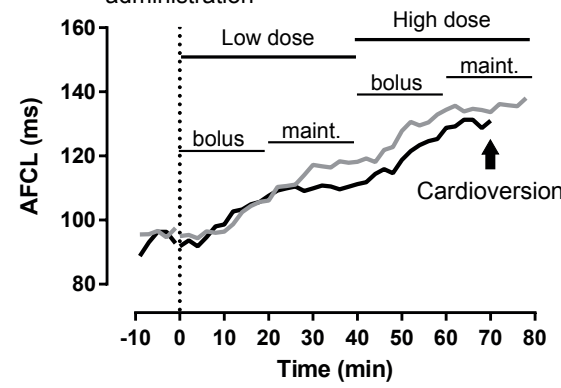

F Vernakalant $\quad 3.7 \mathrm{mg} / \mathrm{kg} \quad 4.5 \mathrm{mg} / \mathrm{kg}$ XAF-1407 $\quad 0.3 \mathrm{mg} / \mathrm{kg} \quad 3.0 \mathrm{mg} / \mathrm{kg}$

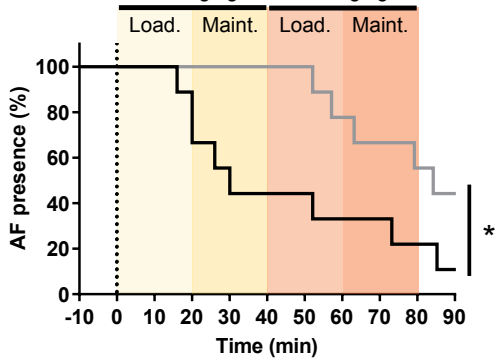

Figure 4. Atrial fibrillation cycle length (AFCL) prolongation and cardioversion during administration of XAF1407 and vernakalant. (A) Left atrial electrograms illustrating the fast and irregular rhythm during AF at baseline and 60 seconds before cardioversion with XAF-1407 and (B) vernakalant. (C) Examples of gradual prolongation of AFCL during the administration of XAF-1407 with cardioversion occurring during the maintenance phase after the dose of $0.3 \mathrm{mg} / \mathrm{kg}$ and (D) $3.0 \mathrm{mg} / \mathrm{kg}$ of XAF-1407. Prolongation of AFCL during the cardioversion attempt with vernakalant in the same animal is depicted for comparison. (E) Comparison of AFCL at baseline ( $\mathrm{n}=9)$ and 60 seconds before cardioversion ( $\mathrm{n}=8$ and $\mathrm{n}=5$ for XAF-1407 and vernakalant, respectively). Mann Whitney test, ${ }^{*} \mathrm{p}<0.05$. (F) XAF-1407 showed higher cardioversion efficacy than vernakalant $\left({ }^{*} \mathrm{p}<0.05\right.$, Log-rank test, $\left.\mathrm{n}=9\right)$. The loading and maintenance phases of the applied dosing regimens are highlighted. 
and vernakalant resulted in gradual prolongation of AFCL (Figure 4A-D). Compared to the administration of vernakalant, the AFCL just prior to cardioversion was shorter for XAF-1407 than for vernakalant (Figure 4E).

A

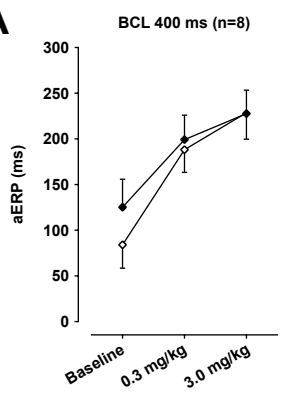

C

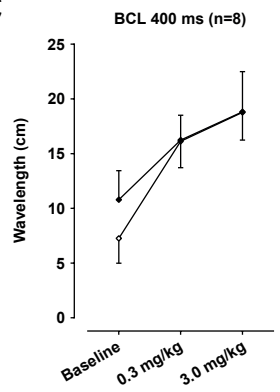

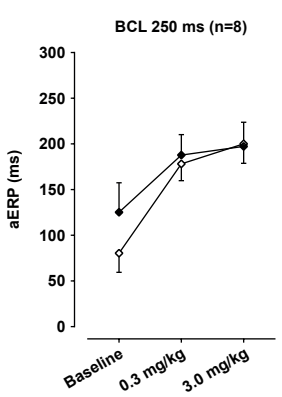

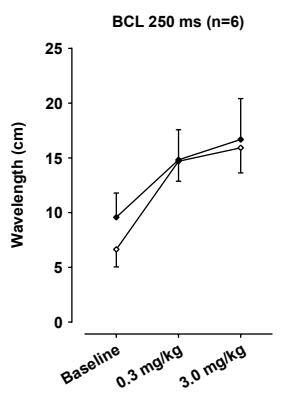

B

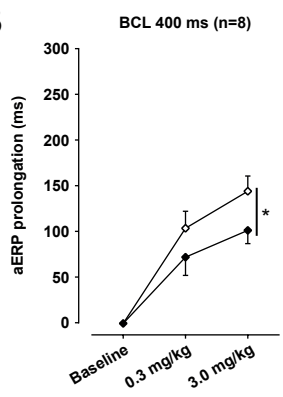

D

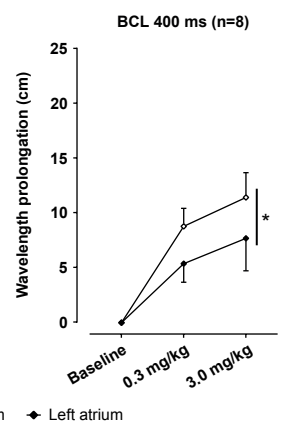

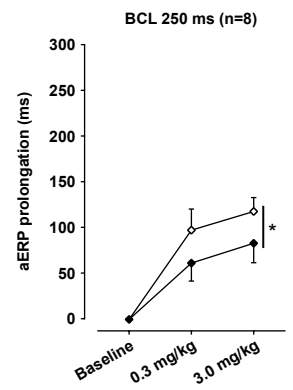

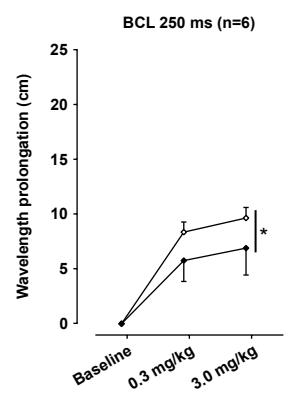

E
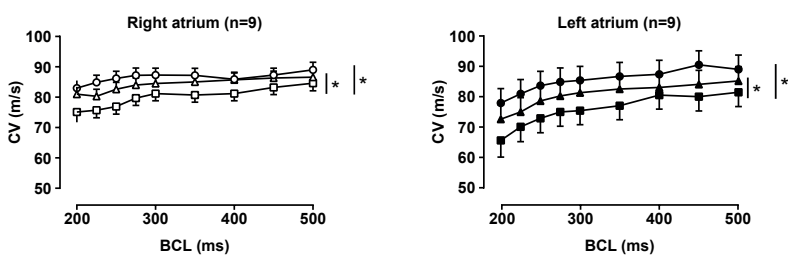

-o- Baseline

$\checkmark-0.3 \mathrm{mg} / \mathrm{kg}$ XAF- 1407 ㅁ. $3.0 \mathrm{mg} / \mathrm{kg}$ XAF-1407

Figure 5. The effect of XAF-1407 on atrial refractoriness, wavelength and conduction velocity during pacing. (A) XAF-1407 induced a dose-dependent prolongation of atrial effective refractory period (aERP). (B) The degree of aERP prolongation was larger in the right atrium. (C) A strong, dose-dependent prolongation of wavelength was observed in both atria during pacing. (D) Similarly, the prolongation of wavelength was more pronounced in right atrium. For aERP and wavelength, the sample sizes are stated in the brackets next to the burst cycle length (BCL). (E) Dose-dependent decrease of conduction velocity was observed in both atria. Presented as estimated mean \pm standard error of the mean. ${ }^{*} \mathrm{p}<0.05$. 
The open chest experiment was performed in nine animals. One animal developed sterile pericarditis and was excluded from the experiment. The measurements of aERPs revealed that the effect of XAF-1407 on atrial refractoriness was generally larger in the RA compared to the LA. The aERPs at baseline were typically shorter in the RA when compared to the LA (Figure $5 \mathrm{~A}$ ). The dose of $0.3 \mathrm{mg} / \mathrm{kg}$ of XAF-1407 caused a strong prolongation in aERP in both RA and LA (Figure 5B). The dose of $3.0 \mathrm{mg} / \mathrm{kg}$ resulted in further prolongation of aERP in both atria. Similarly, the administration of XAF-1407 led to a strong, dose-dependent prolongation of wavelength in both atria (Figure 5C) with a more pronounced effect in the RA (Figure 5D). The administration of XAF-1407 led to a dose-dependent decrease of CV at all cycle lengths in LA and RA with no apparent rate-dependent differences (Figure 5E).

Administration of XAF-1407 had only a modest effect on ventricular hemodynamics (Table 1). The left ventricular (LV) end-diastolic and systolic pressure showed a minor increase. Similarly, there was a small increase in the maximum rate of $L V$ pressure change $\left(\mathrm{dP}_{\mathrm{dt}} \mathrm{t}_{\max }\right)$ after the administration of XAF-1407.

Table 1. Hemodynamic parameters in left ventricle.

\begin{tabular}{llll}
\hline & \multicolumn{1}{c}{ Baseline } & \multicolumn{2}{c}{ XAF-1407 } \\
\cline { 3 - 4 } & \multicolumn{1}{c}{$\mathbf{0 . 3 ~ \mathbf { ~ m g } / \mathbf { k g }}$} & \multicolumn{1}{c}{$\mathbf{3 . 0 ~} \mathbf{~ m g} / \mathbf{k g}$} \\
\hline $\mathrm{P}_{\text {enddia }}, \mathrm{mmHg}$ & $5.19(3.85-10.65)$ & $6.69(5.71-10.27)^{*}$ & $7.84(5.33-10.66)^{*}$ \\
$\mathrm{P}_{\text {sys }}, \mathrm{mmHg}$ & $116(93-125)$ & $120(109-130)$ & $123(114-126)$ \\
$\mathrm{dP} / \mathrm{dt}_{\max }, \mathrm{mmHg} / \mathrm{s}$ & $3672(3105-4334)$ & $4183(3689-4426)$ & $4286(3599-5118)$ \\
\hline
\end{tabular}

Data are presented as median and interquartile range

$\mathrm{P}_{\text {enddia }}$ end-diastolic pressure, $\mathrm{P}_{\text {sys }}$ systolic pressure, $\mathrm{dP} / \mathrm{dt}_{\max }$ peak rate of pressure

${ }^{*} \mathrm{p}<0.05$, compared to baseline, $\mathrm{n}=8$.

During the 1.5 to 2 seconds before pharmacological cardioversion with XAF-1407, AF complexity decreased in both atria (Figures 6 and 7). This period of organization was associated with decreased activation frequency, less electrical dyssynchrony and fewer breakthrough waves. Yet, there was only a minor reduction of AF complexity in the last 10 seconds preceding this rapid organization period, indicating that the decline in AF complexity only occurred just before cardioversion. 
A

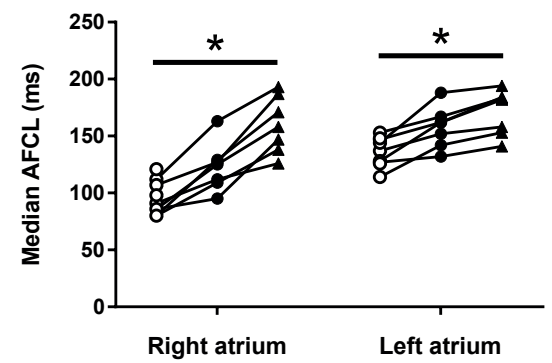

C

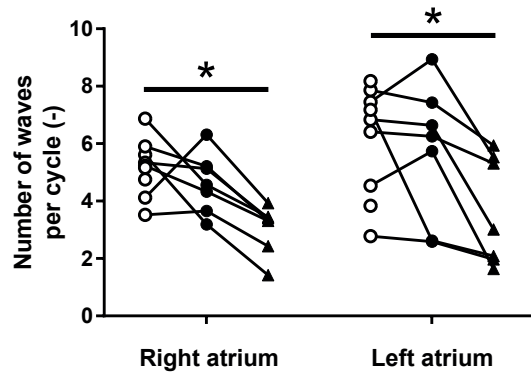

E

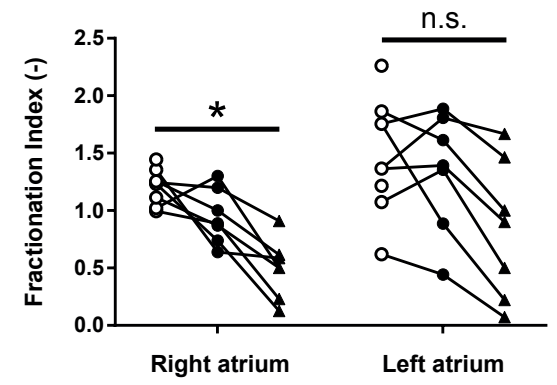

B

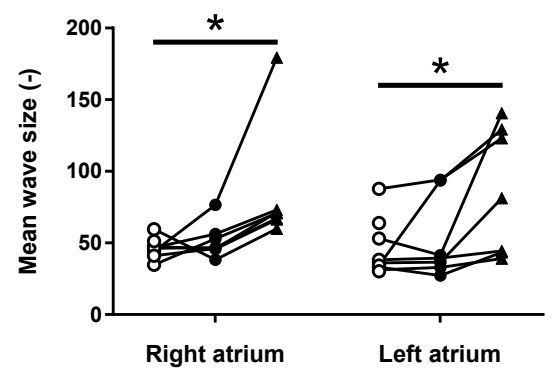

D

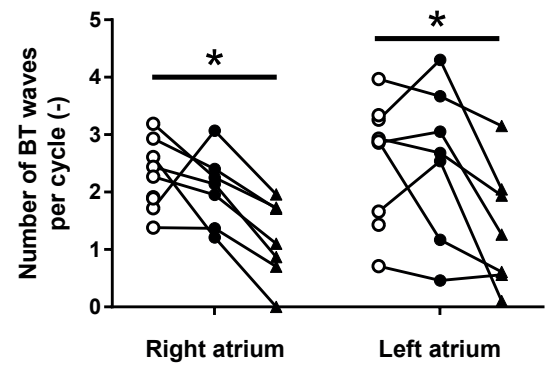

$\mathbf{F}$

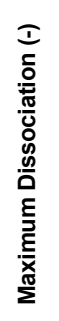

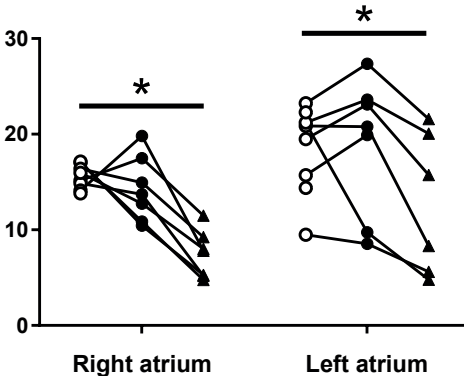

$$
\begin{aligned}
& \text { - Baseline } \\
& \rightarrow 10 \mathrm{~s} \text { before CV } \\
& -2 \mathrm{~s} \text { before CV }
\end{aligned}
$$

Figure 6. XAF-1407 reduces the complexity of atrial fibrillation in the left and right atrium. (A) Median atrial fibrillation cycle length (AFCL), (B) mean wave size, expressed as a number of electrodes assigned to one fibrillatory wave, (C) number of waves per AF cycle, (D) number of breakthrough (BT) waves per AF cycle, (E) fractionation index and (F) maximum wave dissociation. Friedman's test, ${ }^{*} \mathrm{p}<0.05, \mathrm{n}=9$. 
Baseline

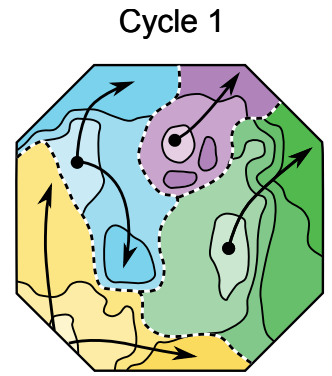

Cycle 2
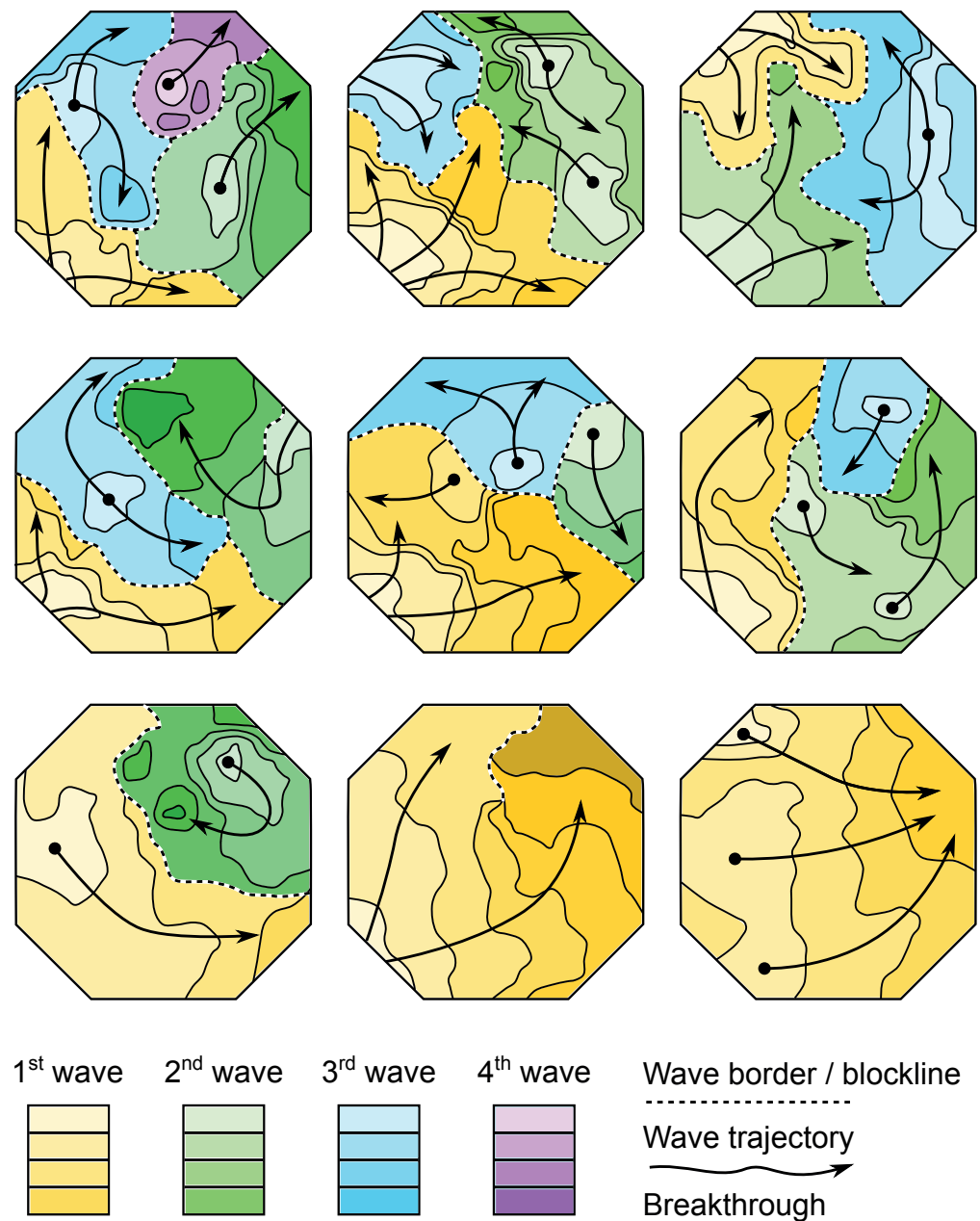
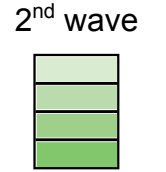

$3^{\text {rd }}$ wave $4^{\text {th }}$ wave

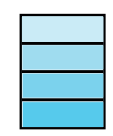

Cycle 3

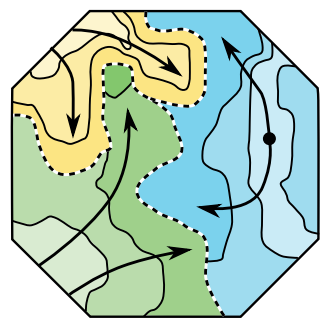

Figure 7. The effect of XAF-1407 on fibrillation patterns. Wave maps of three consecutive beats at baseline, 10 seconds before cardioversion and 2 seconds before cardioversion. Each map displays the territory of individual fibrillation waves within the mapped area of LA free wall during a single AF cycle. The color-coding depicts the sequence of wave appearance. The isochronal lines within waves are separated by $10 \mathrm{~ms}$. AF complexity decreased 1.5 to 2 seconds before pharmacological cardioversion with XAF-1407. However, there was only a moderate reduction in AF complexity in the last 10 seconds preceding this rapid organization period. 


\section{DISCUSSION}

This study demonstrates that selective $\mathrm{I}_{\mathrm{KACh}}$ inhibition by XAF-1407 represents a safe therapeutic strategy for treatment of AF. We showed that selective inhibition of $\mathrm{I}_{\mathrm{KACh}}$ by XAF1407 results in prolongation of aERP and AFCL, leading to AF termination. XAF-1407 caused a modest prolongation of QRS and QTc in the absence of any observed ventricular proarrhythmic effects.

\section{Effect of XAF-1407 on atrial refractoriness}

The aERP prolongation after the administration of XAF-1407 was substantially larger in electrically remodeled atria. This finding supports the hypothesis that constitutively active $\mathrm{I}_{\mathrm{KACh}}$ increases during AF. ${ }^{7}$ Reverse rate dependency in the effect of XAF-1407 was observed in normal atria as well as in electrically remodeled atria. Similar findings have been reported in the goat model of AF for AVE0118, a class III antiarrhythmic drug inhibiting the ultra-rapid delayed rectifier $\left(\mathrm{I}_{\text {Kur }}\right)$ and transient outward $\left(\mathrm{I}_{\mathrm{to}}\right)$ currents. ${ }^{13}$ Prolongation of aERP has also been reported for XAF-1407 in an equine model of AF. ${ }^{8}$

The effect of XAF-1407 on atrial refractoriness in normal atria was relatively large and unexpected. To investigate the possibility that a high vagal tone in normal goats augments the aERP prolongation after $\mathrm{I}_{\mathrm{KACh}}$ inhibition, we performed an additional experiment. In two goats without AF-induced electrical remodeling we measured aERPs after vagal inhibition by atropine, followed by $\mathrm{I}_{\mathrm{KACh}}$ inhibition by XAF-1407. Although the number of animals in this experimental group was small, the absence of any aERP shortening after the infusion of atropine strongly suggests that the observed effect of XAF-1407 on refractoriness in normal atria was not caused by high vagal tone. We therefore propose that the effect of XAF-1407 on atrial refractoriness in normal goat atria can be explained by constitutively active $\mathrm{I}_{\mathrm{KACh}}$ even without AF-induced electrical remodeling. Such finding of constitutively active $\mathrm{I}_{\mathrm{KACh}}$ has been reported for normal canine atria ${ }^{22}$ but is clearly not the case in human atria in the absence of AF-induced remodeling. ${ }^{7}$

The effect of $\mathrm{I}_{\text {KACh }}$ inhibition on atrial refractoriness was larger in the RA than in the LA, with baseline aERPs in the RA being generally shorter after 4 weeks of pacing-induced AF. The administration of $0.3 \mathrm{mg} / \mathrm{kg}$ of XAF-1407 decreased the right-to-left aERP gradient and the gradient entirely disappeared after the dose of $3.0 \mathrm{mg} / \mathrm{kg}$. It has been shown that the gradient in $\mathrm{I}_{\mathrm{KACh}}$ density between the atria can differ among species. ${ }^{23}$ Therefore, we suggest that the larger effect of XAF-1407 in the RA might be caused by a stronger contribution of $\mathrm{I}_{\mathrm{KACh}}$ to repolarization in goat RA. Compared to XAF-1407, the effect of vernakalant on 
atrial refractoriness was less pronounced, as described by previous studies in the goat model of pacing-induced AF. ${ }^{10,11}$ Selective $\mathrm{I}_{\mathrm{KACh}}$ inhibition therefore appears to have a strong effect on atrial refractoriness in goats.

\section{Effect of XAF-1407 on atrial conduction and wavelength}

We observed a moderate dose-dependent reduction of conduction velocity during the infusion of XAF-1407, indicating a class I effect of the drug. This effect was possibly mediated through a depolarization of the resting membrane potential, resulting in the decreased number of available sodium channels. The effect was comparable between RA and LA. In comparison, even more pronounced, dose-dependent decrease in atrial conduction velocity was observed for vernakalant, which is known to exhibit a class I effect due to the inhibition of the fast sodium current. ${ }^{11,17}$

The observed strong effect of XAF-1407 on atrial refractoriness and its moderate effect on atrial conduction velocity resulted in substantial prolongation of atrial wavelength in both atria which is a finding that confirms the proposed class III action of the compound. In contrast, in a previous study we observed that vernakalant does not lead to a prolongation of atrial wavelength in electrically remodeled atria. ${ }^{11}$ Although the direct comparison of the degree of wavelength prolongation is not possible because the measurements with XAF-1407 were performed under general anesthesia while the measurements with vernakalant were performed in awake animals, our findings indicate strong effect of XAF-1407 on prolongation of atrial wavelength, suggesting strong efficacy of the drug to terminate AF.

\section{Cardioversion efficacy of XAF-1407}

The efficacy of XAF-1407 to cardiovert AF (8 out of 9, 89\%) was comparable to the efficacy reported in an equine model of persistent $\mathrm{AF}$ ( $80 \%$ and $70 \%$ after 11 and 17 days of persistent AF, respectively). ${ }^{8}$ It was also similar to the efficacy of previously tested class III antiarrhythmic drugs in the same goat model of pacing-induced AF. The administration of $\mathrm{I}_{\text {Kur }}$ and $\mathrm{I}_{\text {to }}$ inhibitor AVE0 118 resulted in cardioversion rate of $63 \%$ when AF was induced for $53 \pm 19$ days ${ }^{13}$ and $100 \%$ when AVE0118 was administered in combination with a rapid delayed rectifier current $\left(\mathrm{I}_{\mathrm{Kr}}\right)$ inhibitors dofetilide or ibutilide in goats with AF induced for $57 \pm 7$ days. ${ }^{14}$ For the inward rectifier potassium current $\left(\mathrm{I}_{\mathrm{K} 1}\right)$ inhibitor PA- 6 the cardioversion rate after 3 weeks of AF was $83 \% .{ }^{12}$ Although direct comparison of cardioversion efficacies within the same goat model is not straightforward due to the different time points investigated and the dosing regimens used, our results indicate that $\mathrm{I}_{\mathrm{KACh}}$ inhibition is a promising therapeutic strategy for treatment of $\mathrm{AF}$.

The effect of the vehicle $(0.1 \mathrm{~mol} / \mathrm{l}$ sodium acetate buffer $)$ on cardioversion efficacy has not been investigated in this study. However, previous experimental study with XAF-1407 reported a $0 \%$ cardioversion rate after intravenous administration of the vehicle only. ${ }^{8}$ 
Considering the similar time point of AF development, we argue that the efficacy of XAF-1407 to cardiovert AF is superior to vernakalant. The efficacy of vernakalant to cardiovert AF (5 out of $9,56 \%$ ) was identical to the previous study in goats with 11 days of AF in which the same cardioversion rate $(56 \%)$ was achieved. ${ }^{11}$

\section{XAF-1407 slows down the conduction of sinoatrial and atrioventricular node}

It has been reported that $\mathrm{I}_{\mathrm{KACh}}$ channels are expressed in sinoatrial $(\mathrm{SA})^{5}$ and atrioventricular (AV) node. ${ }^{6}$ In the present study, we show that $\mathrm{I}_{\mathrm{KACh}}$ inhibition by XAF-1407 had mild bradycardic effect during sinus rhythm in normal atria at a dose of $0.3 \mathrm{mg} / \mathrm{kg}$. A stronger deceleration of sinus rhythm in electrically remodeled atria was observed after the dose of $3.0 \mathrm{mg} / \mathrm{kg}$. To study the effect of $\mathrm{I}_{\mathrm{KACh}}$ inhibition on the AV node, we investigated the shift in Wenckebach cycle length, showing that infusion of XAF-1407 markedly increased the median Wenckebach cycle length. This observation is also in agreement with the observed prolongation of PQ interval after the infusion of $3.0 \mathrm{mg} / \mathrm{kg}$ of XAF-1407. Contrary to our findings, enhanced AV node conduction and small increase in heart rate were observed when XAF-1407 was administered in horses. ${ }^{8}$ The different effect between goats and horses might be possibly explained by a species-specific expression of ion channels in the SA and AV node. The design of our study did not allow us to investigate the mechanisms underlying the changes in the nodal function due to the administration of XAF-1407 in goats. The observed effect on SA and AV node does not have to be caused by the direct effect of the compound on the nodal cells, but it might be also the result of the coupling of nodal cells with atrial cardiomyocytes or due to changes in electrotonic interactions. ${ }^{24}$

Our data show that $\mathrm{I}_{\mathrm{KACh}}$ inhibition by XAF-1407 affects the function of the SA and AV nodes. In the context of AF, deceleration of sinus rhythm can potentially be seen as an adverse effect. Slowing down of sinus rhythm increases the probability of dispersion of atrial refractoriness and occurrence of atrial ectopy, both of which potentially facilitate reinitiation of AF. By contrast, decreasing of AV nodal conduction during AF may potentially be beneficial because it contributes to the reduction of ventricular rate during AF, thereby possibly preventing tachycardia-induced cardiomyopathy.

\section{XAF-1407 decreases AF complexity}

The progressive character of $\mathrm{AF}$-induced remodeling makes the fibrillation pattern more complex and the arrhythmia more persistent. ${ }^{15}$ The current study showed a decrease of AF complexity 1.5 to 2 seconds prior to cardioversion during XAF-1407 administration. This time period was associated with a decrease in the total number of waves and breakthrough waves per AF cycle, as well as with an increase of wave size. A similar decrease of AF complexity has 
been described in the same animal model for the $\mathrm{I}_{\mathrm{K} 1}$ inhibitor PA- $6 .{ }^{12}$ Only limited changes in AF pattern- and complexity-related parameters were observed in the period of 2 to 12 seconds prior to cardioversion. This finding indicates that the decrease of AF complexity 1.5 to 2 seconds prior to cardioversion is associated with the cardioversion process itself rather than caused by the $\mathrm{I}_{\mathrm{KACh}}$ inhibition by XAF-1407. Similar finding has been reported for a class I antiarrhythmic drug cibenzoline in which an abrupt increase of AFCL and a reduced occurrence of fractionated electrograms in the last beats preceding cardioversion has been observed..$^{25}$

\section{Ventricular safety of XAF-1407}

A modest effect of XAF-1407 on LV pressure was observed in anesthetized animals. The administration of XAF-1407 resulted in a small increase in end-diastolic pressure and a small increase of LV systolic pressure and its maximum derivative $\left(\mathrm{dP} / \mathrm{dt}_{\max }\right)$. Due to the fact that the hemodynamic measurements were performed during an open-chest procedure, their interpretation should be performed with caution.

Administration of $0.3 \mathrm{mg} / \mathrm{kg}$ of XAF-1407 was not associated with prolongation of QTc and QRS and the prolongation of both QTc and QRS after the infusion of $3.0 \mathrm{mg} / \mathrm{kg}$ of XAF-1407 was only limited. The small effect of XAF-1407 on QTc prolongation together with an absence of ventricular proarrhythmic events (extrasystoles, non-sustained ventricular tachycardia) during its administration indicate ventricular safety of the drug. Considering the high selectivity of XAF-1407 for $\mathrm{I}_{\mathrm{KACh}}$ channels $^{8}$ and the fact that the median prolongation of QTC and QRS did not exceed 10\%, the results suggest that XAF-1407 is a selective inhibitor of $\mathrm{I}_{\mathrm{KACh}}$ in the goat model of AF.

\section{$I_{\text {KACh }}$ as a target for AF treatment in humans}

There are several studies that investigated $\mathrm{I}_{\mathrm{KACh}}$ inhibition as a target for treatment of $\mathrm{AF}$ in humans. ${ }^{26,27}$ Modestly selective $\mathrm{I}_{\mathrm{KACh}}$ inhibitors AZD2927 and A7071 increased atrial refractoriness and were associated with $\mathrm{AF}$ termination in dogs. ${ }^{27}$ However, these positive results could not been replicated in atrial flutter patients. ${ }^{27}$ Similarly, the highly-selective $\mathrm{I}_{\mathrm{KACh}}$ blocker BMS914392 did not show any reduction in AF burden in patients with paroxysmal $\mathrm{AF}$, although it could terminate $\mathrm{AF}$ in a dog model. ${ }^{26}$

In contrast to BMS914392, AZD2927 and A7071, XAF-1407 has been shown to inhibit both $\mathrm{K}_{\mathrm{ir}} 3.1 / \mathrm{K}_{\mathrm{ir}} 3.4$ heterotetramers, and $\mathrm{K}_{\mathrm{ir}} 3.4 / \mathrm{K}_{\mathrm{ir}} 3.4$ homotetramers with similar potency which has been shown to increase the antiarrhythmic efficacy of the compound. ${ }^{8}$ Nevertheless, there are no data available yet on XAF-1407 in humans to verify the antiarrhythmic efficacy of XAF1407 in patients with AF. 


\section{LIMITATIONS}

The current study represents an early time point in the development of AF-induced remodeling which is known to gradually increase AF stability over a time course of months. ${ }^{15} \mathrm{~A}$ loss of efficacy during AF progression has been observed for several antiarrhythmic drugs in the goat model of AF. ${ }^{15}$ To assess the efficacy of XAF-1407 in later time points of AF-induced remodeling, follow-up studies with longer period of AF maintenance will be necessary.

It has been shown that $\mathrm{I}_{\mathrm{KACh}}$ contributes to the increased inward-rectifying potassium current in patients with chronic AF. Although our results indicate that $I_{\mathrm{KACh}}$ is also constitutively active in normal goat atria, our data does not preclude the effectiveness of $\mathrm{I}_{\mathrm{KACh}}$ blockade as an antiarrhythmic treatment. Indeed, if $\mathrm{I}_{\mathrm{KACh}}$ inhibition selectively prolongs atrial action potential duration in the absence of AF-induced electrical remodeling, its antiarrhythmic properties could be effective in a wider range of clinical settings and patient populations.

The work by Fenner et al. demonstrated that XAF-1407 is a highly selective inhibitor of $\mathrm{I}_{\mathrm{KACh}}$ channels. ${ }^{8}$ The goat model of pacing-induced AF that was used in this study did not allow us to investigate selectivity of the drug to different ionic currents. Therefore, it was not possible for us to describe the mechanisms underlying some of the observed effects of XAF-1407 on cardiac electrophysiology. The large effect of XAF-1407 on aERP in non-remodeled atria, its class I effect indicated by the decreased atrial conduction as well as the changes in the nodal function and the effect on ventricular electrophysiology described by the prolongation of QRS and QTc can be possibly explained by a non-specific effect of XAF-1407. Additional patch clamp experiments would be necessary to explain these effects of XAF-1407 on cardiac electrophysiology.

\section{CONCLUSION}

Our study demonstrates that $\mathrm{I}_{\mathrm{KACh}}$ inhibitor XAF-1407 has high efficacy to terminate AF, with pronounced atrial selectivity. Our study in the goat model of AF thus indicates that $\mathrm{I}_{\mathrm{KACh}}$ represents a promising therapeutic target for treatment of AF. 


\section{REFERENCES}

1. Kirchhof P, Benussi S, Kotecha D, Ahlsson A, Atar D, Casadei B, et al. 2016 ESC Guidelines for the management of atrial fibrillation developed in collaboration with EACTS. Europace 2016;18:16091678.

2. Schotten U, Verheule S, Kirchhof P, Goette A. Pathophysiological mechanisms of atrial fibrillation: a translational appraisal. Physiol Rev 2011;91:265-325.

3. Milnes JT, Madge DJ, Ford JW. New pharmacological approaches to atrial fibrillation. Drug Discov Today 2012;17:654-659.

4. Koumi S, Wasserstrom JA. Acetylcholine-sensitive muscarinic $\mathrm{K}^{+}$channels in mammalian ventricular myocytes. Am J Physiol 1994;266:H1812-H1821.

5. Noma A, Trautwein W. Relaxation of the ACh-Induced Potassium Current in the Rabbit Sinoatrial Node Cell. Pflügers Arch 1978;377:193-200.

6. Sakmann B, Noma A, Trautwein W. Acetylcholine activation of single muscarinic $\mathrm{K}^{+}$channels in isolated pacemaker cells of the mammalian heart. Nature 1983;303:250-253.

7. Dobrev D, Friedrich A, Voigt N, Jost N, Wettwer E, Christ T, et al. The G protein-gated potassium current $\mathrm{I}(\mathrm{K}, \mathrm{ACh})$ is constitutively active in patients with chronic atrial fibrillation. Circulation 2005;112:3697-3706.

8. Fenner MF, Carstensen H, Nissen SD, Hesselkilde EZ, Lunddahl C, Jensen MA, et al. Effect of selective $\mathrm{I}_{\mathrm{K}, \mathrm{Ach}}$ inhibition by XAF-1407 in an equine model of tachypacing-induced persistent atrial fibrillation. BrJ Pharmacol 2020;177:3778-3794.

9. Wijffels MCEF, Kirchhof CJHJ, Dorland R, Allessie MA. Atrial Fibrillation Begets Atrial Fibrillation: A Study in Awake Chronically Instrumented Goats. Circulation 1995;92:1954-1968.

10. van Hunnik A, Nasrallah H, Lau DH, Kuiper M, Verheule S, Schotten U. Vernakalant does not alter early repolarization or contractility in normal and electrically remodelled atria. Europace 2018;20:140-148.

11. van Hunnik A, Lau DH, Zeemering S, Kuiper M, Verheule S, Schotten U. Antiarrhythmic effect of vernakalant in electrically remodeled goat atria is caused by slowing of conduction and prolongation of postrepolarization refractoriness. Heart Rhythm 2016;13:964-972.

12. Ji Y, Varkevisser R, Opacic D, Bossu A, Kuiper M, Beekman JDM, et al. The inward rectifier current inhibitor PA-6 terminates atrial fibrillation and does not cause ventricular arrhythmias in goat and dog models. Br J Pharmacol 2017; 174:2576-2590.

13. Blaauw Y, Gogelein H, Tieleman RG, van Hunnik A, Schotten U, Allessie MA. "Early" class III drugs for the treatment of atrial fibrillation: efficacy and atrial selectivity of AVE0118 in remodeled atria of the goat. Circulation 2004;110:1717-1724.

14. Blaauw Y, Schotten U, van Hunnik A, Neuberger HR, Allessie MA. Cardioversion of persistent atrial fibrillation by a combination of atrial specific and non-specific class III drugs in the goat. Cardiovasc Res 2007;75:89-98.

15. Verheule S, Tuyls E, van Hunnik A, Kuiper M, Schotten U, Allessie M. Fibrillatory conduction in the atrial free walls of goats in persistent and permanent atrial fibrillation. Circ Arrhythm Electrophysiol 2010;3:590-599. 
16. Schotten U, Duytschaever M, Ausma J, Eijsbouts S, Neuberger HR, Allessie M. Electrical and contractile remodeling during the first days of atrial fibrillation go hand in hand. Circulation. 2003;107:1433-1439.

17. Fedida D, Orth PM, Chen JY, Lin S, Plouvier B, Jung G, et al. The mechanism of atrial antiarrhythmic action of RSD1235. J Cardiovasc Electrophysiol 2005;16:1227-1238.

18. Camm AJ. The vernakalant story: how did it come to approval in Europe and what is the delay in the U.S.A? Curr Cardiol Rev 2014;10:309-314.

19. Mohan NK, Niyogi D, Singh HN. Analysis of relationship between Q-T and R-R interval in the electrocardiogram of goats. Indian J Anim Sci 2009;79:362-365.

20. Zeemering S, Maesen B, Nijs J, Lau DH, Granier M, Verheule S, et al. Automated quantification of atrial fibrillation complexity by probabilistic electrogram analysis and fibrillation wave reconstruction. Conf Proc IEEE Eng Med Biol Soc 2012;2012:6357-6360.

21. Wiener N, Rosenblueth A. The mathematical formulation of the problem of conduction of impulses in a network of connected excitable elements, specifically in cardiac muscle. Arch Inst Cardiol Mex. 1946;16:205-265.

22. Voigt N, Maguy A, Yeh YH, Qi X, Ravens U, Dobrev D, et al. Changes in $I_{K, A C h}$ single channel activity with atrial tachycardia remodelling in canine atrial cardiomyocytes. Cardiovasc Res 2008;77:35-43.

23. Anumonwo JM, Lopatin AN. Cardiac strong inward rectifier potassium channels. J Mol Cell Cardiol 2010;48:45-54.

24. Jalife J. Mutual entrainment and electrical coupling as mechanisms for synchronous firing of rabbit sino-atrial pace-maker cells. J Physiol 1984;356:221-243.

25. Shan Z, van der Voort PH, Blaauw Y, Duytschaever M, Allessie MA. Fractionation of electrograms and linking of activation during pharmacologic cardioversion of persistent atrial fibrillation in the goat. J Cardiovasc Electrophysiol 2004;15:572-580.

26. Podd SJ, Freemantle N, Furniss SS, Sulke N. First clinical trial on specific $I_{\text {KACh }}$ blocker shows no reduction in atrial fibrillation burden in patients with paroxysmal atrial fibrillation: pacemaker assessment of BMS 914392 in patients with paroxysmal atrial fibrillation. Europace 2016;18:340346.

27. Walfridsson H, Anderson KP, Anfinsen OG, Berggren A, Frison L, Jensen S, et al. Is the acetylcholine-regulated inwardly rectifying potassium current a viable antiarrhythmic target? Translational discrepancies of AZD2927 and A7071 in dogs and humans. Europace 2015;17:473482. 



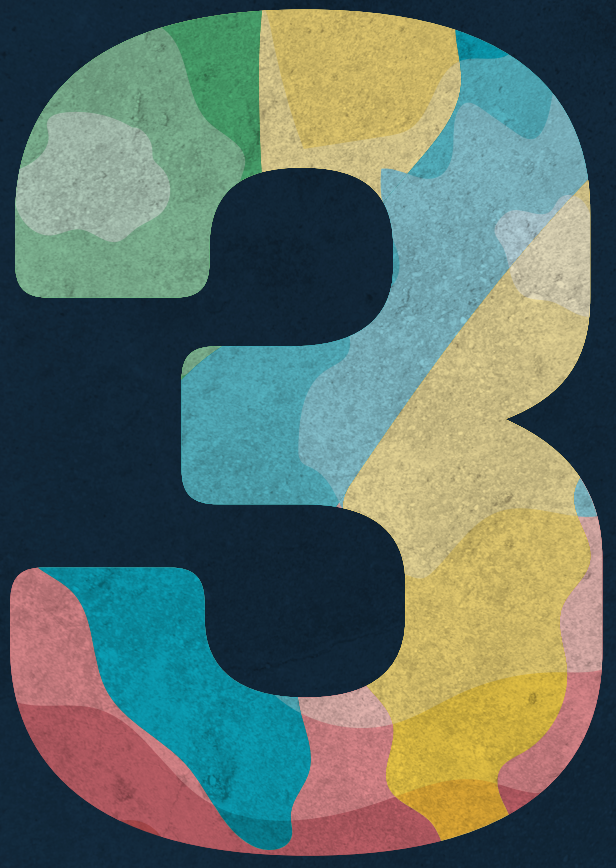




\section{Effective termination of atrial fibrillation by SK channel inhibition is associated with a gradual cycle length increase in contrast to a sudden organization of fibrillatory conduction}

Giulia Gatta, Vladimír Sobota, Carlotta Citerni, Jonas Goldin Diness, Ulrik S. Sørensen, Thomas Jespersen, Bo Hjorth Bentzen, Stef Zeemering, Marion Kuiper, Sander Verheule, Ulrich Schotten and Arne van Hunnik

Accepted for publication in Europace 


\section{ABSTRACT}

Background: Pharmacological termination of atrial fibrillation (AF) remains a challenge due to limited efficacy and potential ventricular proarrhythmic effects of antiarrhythmic drugs. Small conductance calcium-activated potassium (SK) channels are proposed as atrial-specific targets in the treatment of AF. Here, we investigated the effects of the new SK channel inhibitor AP14145.

Methods: Eight goats were implanted with pericardial electrodes for induction of AF (30 days). In an open chest study, the atrial conduction velocity (CV) and effective refractory period (ERP) were measured during pacing. High-density mapping of both atrial free walls was performed during AF and conduction properties were assessed. All measurements were performed at baseline and during AP14145 infusion $(10 \mathrm{mg} / \mathrm{kg} / \mathrm{h}, \mathrm{n}=1$ or $20 \mathrm{mg} / \mathrm{kg} / \mathrm{h}, \mathrm{n}=6)$.

Results: At an infusion rate of $20 \mathrm{mg} / \mathrm{kg} / \mathrm{h}$, AF terminated in $5 / 6$ goats. AP14145 profoundly increased ERP and reduced CV during pacing. AP14145 increased spatiotemporal instability of conduction at short pacing cycle lengths. AF cycle length and path length (AF cycle length $x \mathrm{CV}$ ) underwent a strong dose-dependent prolongation. CV during AF remained unchanged and conduction patterns remained complex until the last seconds before AF termination, during which a sudden and profound organization of fibrillatory conduction occurred.

Conclusions: AP14145 provided a successful therapy for termination of persistent AF in goats. During AF, AP14145 caused an ERP and AF cycle length prolongation. AP14145 slowed CV during fast pacing but did not lead to a further decrease during AF. Termination of AF was preceded by an abrupt organization of AF with a decline in the number of fibrillation waves. 


\section{INTRODUCTION}

Currently available antiarrhythmic drugs (AADs) for the treatment of atrial fibrillation (AF) lose their efficacy with AF progression and mainly convert recent-onset AF that lasts less than 48 hours. ${ }^{1}$ The lack of atrial selectivity limits the use of more aggressive antiarrhythmic therapy, as many $\mathrm{AADs}$ lead to ventricular proarrhythmia, particularly in patients with structural heart disease. A more effective and safe AF treatment might be achieved by targeting ion channels that are predominantly expressed in the atrium. The small conductance calcium-activated potassium ( $\mathrm{SK}$ or $\mathrm{K}_{\mathrm{Ca}} 2$ ) channel has been proposed as such an atrial-selective target. Three subtypes of this ion channel have been identified, of which SK2 and SK3 are abundant in the human atria. SK channel opening is triggered by an increase in the intracellular $\mathrm{Ca}^{2+}$ concentration and therefore affects the action potential plateau and terminal repolarization in a $\mathrm{Ca}^{2+}$-dependent manner. ${ }^{2}$ Inhibition of SK channels leads to prolongation of the action potential (AP) and atrial effective refractory period (ERP) in human muscle bundles ${ }^{2}$ and shortens AF paroxysms. ${ }^{3,4}$ In the canine left atrium, SK channel expression levels and SK current densities were enhanced after 7 days of AF-induced electrical remodeling. ${ }^{3}$ Indeed, it has been shown that selective SK channel inhibition could prolong AP duration and ERP in human atrial muscle bundles from chronic AF patients. ${ }^{2}$

In addition, it has been reported that SK channel inhibition may lead to a small depolarization of the resting membrane potential which was associated with a reduced AP upstroke velocity $(\mathrm{dV} / \mathrm{dt})$ and amplitude in human cardiomyocytes. ${ }^{2,5}$ Moreover, the combination of a selective SK channel inhibitor (ICA) and sodium channel blockers synergistically prolonged ERP. ${ }^{6}$ This reduction of excitability after SK channel inhibition, functionally a class I effect, could potentially act as an additional antiarrhythmic mechanism.

Recently, a new compound, AP14145, was developed that showed selective SK channel inhibiting properties in HEK293 cell lines. ${ }^{4,7}$ AP14145 also exhibited potential for AF treatment, as it was able to terminate sustained $\mathrm{AF}$ and protect against its reinduction in a model where the clinically available drug vernakalant failed to terminate $\mathrm{AF}^{4}$ Here, we have studied the antiarrhythmic mechanisms of SK channel inhibition by AP14145 during AF and regular rhythms in a goat model of persistent AF. We performed bi-atrial high-density mapping to obtain insight into the effects on atrial conduction and termination of AF, including the potential class I effect of AP14145 during AF. 


\section{MATERIALS AND METHODS}

\section{Animal model and surgical procedures}

All animal experiments were conducted according to both the Dutch (license number AVD107002016782) and the European regulations on animal experimentation. Eight goats were anesthetized (induction: sodium thiopental, $10 \mathrm{mg} / \mathrm{kg}$; maintenance: sufentanyl, $6 \mu \mathrm{g} / \mathrm{kg} / \mathrm{h}$ and propofol, $10 \mathrm{mg} / \mathrm{kg} / \mathrm{h}$ ) and implanted with pericardial electrodes above the left atrium (LA) and with a stimulator for AF induction (Medtronic Itrel ${ }^{\circledR}$, Medtronic, Minneapolis, Minnesota, USA). After recovery from surgery, AF was maintained by $50 \mathrm{~Hz}$ pacing at $10 \mathrm{~V}, 1 \mathrm{~s}$ on, $4 \mathrm{~s}$ off.

A terminal experiment was performed after 30 days of AF maintenance under general anesthesia (induction: sodium thiopental $10 \mathrm{mg} / \mathrm{kg}$; maintenance: sufentanyl $6 \mu \mathrm{g} / \mathrm{kg} / \mathrm{h}$, propofol $10 \mathrm{mg} / \mathrm{kg} / \mathrm{h}$ and rocuronium $0.3 \mathrm{mg} / \mathrm{kg} / \mathrm{h}$ ). Standard Einthoven ECG leads were recorded. Hemodynamic parameters were measured by a pressure-tip catheter (Sentron Europe BV, Roden, The Netherlands) in the left ventricle. A left-sided thoracotomy exposed the heart for electrophysiological measurements. Two mapping electrode arrays, each consisting of 249 unipolar electrodes (2.4 mm electrode distance), were placed on right atrial (RA) and LA free wall. Mapping electrodes were kept in a fixed position during the whole experiment. Signals were recorded at a sampling frequency of $1039 \mathrm{~Hz}$. Two defibrillation catheters were placed, one in the RA cavity and one in the coronary sinus, to allow internal direct current (DC) defibrillation. Goats were anticoagulated with $2500 \mathrm{IU} / \mathrm{h}$ of heparin. One hour of stabilization was allowed before the start of the experimental protocol.

\section{Experimental protocol}

Stage 1, AF stability was assessed over a 30-minute period. Unipolar atrial electrograms were recorded continuously in $60 \mathrm{~s}$ periods during the whole period. Next, AF was terminated by a DC shock ( $\leq 20$ J, Physio-Control Lifepak 9B, Medtronic, Minneapolis, Minnesota, USA) and ERP and conduction velocities $(\mathrm{CV})$ were assessed during pacing. The $\mathrm{CV}$ was measured by an S1-S1 protocol, applying $2 x$ threshold, 40 cycles at a range of cycle lengths $(450,400$, 350, 300, 275, 250, $225 \mathrm{~ms}$ ). ERP was measured using an S1-S2 protocol (8:1 ratio, 4x threshold) at 250 and $400 \mathrm{~ms}$ cycle length. The S2 was incremented by 2 ms until capture was observed. The last uncaptured beat was considered as the ERP. AF was electrically terminated if $\mathrm{AF}$ was induced at one of the intermediate measurements to allow full accomplishment of the experimental protocol. After completion of the S1-S1 and S1-S2 measurements, AF was reinduced and sustained for 30 minutes. 
Stage 2 (Figure 1C), the stability of AF was challenged by the SK channel blocker AP14145 (Acesion Pharma, Copenhagen, Denmark). One goat was excluded from AP14145 infusion due to lack of sustained AF, and in one goat AP14145 was infused at a rate of $10 \mathrm{mg} / \mathrm{kg} / \mathrm{h}$ for 45 minutes, reaching a cumulative dose of $7.5 \mathrm{mg} / \mathrm{kg}$. In the remaining 6 goats with sustained AF, AP14145 was infused at a rate of $20 \mathrm{mg} / \mathrm{kg} / \mathrm{h}$ for 45 minutes. AF stability was monitored until a cumulative dose of $15 \mathrm{mg} / \mathrm{kg}$ was reached. Unipolar atrial electrograms were recorded during the whole period of drug infusion. AF was reinduced by automated burst pacing $(50 \mathrm{~Hz}, 1 \mathrm{~s}, 10 \mathrm{~mA})$ whenever sinus rhythm occurred during the 45-minute period of drug infusion.

Stage 3, at 45 minutes, the AF reinduction protocol was switched off. AP14145 infusion was continued at a rate of $20 \mathrm{mg} / \mathrm{kg} / \mathrm{h}$ for the next 15 minutes. If AF did not spontaneously terminate at this point, it was electrically terminated by a DC shock. The S1-S1 and S1S2 measurements of CV and ERP were performed as described above within the 15-minute period. A graphical representation of the outline of the experimental design and measurements is depicted in Figure 1B-D.

\section{Data analysis}

The limb leads and left ventricular pressure were stored and analyzed in IDEEQ software (Instrument Development Engineering and Evaluation, Maastricht University, Maastricht, The Netherlands). Recordings of unipolar atrial electrograms of 60 seconds acquired at the time points of $0,5,10,15,20,25$ and 30 minutes after the start of the drug infusion were used for analysis. Atrial electrograms were analyzed offline using a custom-made algorithm (MATLAB, The MathWorks, Inc., Natick, Massachusetts, USA) based on a probabilistic approach. ${ }^{8}$ This algorithm identifies local deflections/activation times in the unipolar electrograms. AF cycle length, CV during AF and fibrillation waves were computed based on these activation times. ${ }^{8}$ Waves were defined by clusters of activation times that were connected in space and time by an apparent $\mathrm{CV}$ of $\geq 20 \mathrm{~cm} / \mathrm{s}$. A fibrillation wave was classified as breakthrough if the starting point occurred within the mapping array. In certain instances, multiple starting points are located in close proximity, potentially originating from a rugged wavefront or transmural conduction. To avoid overestimation of the number of wavefronts, territories of starting points with $>90 \%$ overlap were merged to a single wave and wave type was assigned to the earliest activation. Within waves, the $\mathrm{CV}$ was calculated for each activation by fitting a plane through the spatially neighboring activations. Waves of $<3$ electrodes were excluded from the analysis. The $5^{\text {th }}$ percentile of the AF cycle length distribution was used as a surrogate measure for ERP during AF. The wavelength during pacing was defined as the product of CV and ERP. The path length during $\mathrm{AF}$ was defined as the product of $\mathrm{CV}$ and $\mathrm{AF}$ cycle length. 


\section{Statistics}

Statistical analysis was performed using IBM SPSS Statistics for Macintosh, version 25 (IBM Corp., Armonk, New York, USA). Single repeated parameters were tested with a paired sample t-test. The dose- or time-dependent effects on AF properties and pacing cycle length dependent effects on $\mathrm{CV}$ were tested with a linear mixed-effects model using a diagonal covariance structure with atrium, cumulative dose and cycle length considered as fixed variables and animal taken as a random variable. Results are reported as mean \pm standard deviation (SD). A p-value $<0.05$ was considered statistically significant.

\section{RESULTS}

\section{The effects of AP14145 on AF stability and global ECG parameters}

One goat did not develop stable AF after 30 days of burst pacing and was excluded for the rest of the study. All sustained AF goats remained in stable AF in the 30-minute window after all surgical manipulations and prior to drug infusion. AF terminated in 5 out of 7 goats at a range of $3.7-9.7 \mathrm{mg} / \mathrm{kg}$ of AP14145, as shown in Figure $2 \mathrm{~A}$. Of the two goats that did not terminate AF, one had an infusion rate of $10 \mathrm{mg} / \mathrm{kg} / \mathrm{h}$ and the other a rate of $20 \mathrm{mg} / \mathrm{kg} / \mathrm{h}$. In 4 out of 5 goats that cardioverted, AF became very unstable as demonstrated by the multiple $\mathrm{AF}$ inductions that were required to maintain $\mathrm{AF}$ (Figure 2A). RR intervals did not change during sinus rhythm (Figure 2A) nor during AF (data not shown). ECG parameters were assessed during regular pacing to avoid necessity of corrections for rate. No changes in the QRS duration or in the QT time were observed in the applied range of pacing cycle lengths (Figure 2B). Small trends towards increased left ventricular systolic pressure or in $\mathrm{dP} / \mathrm{dt}_{\text {max }}$ were observed after the administration of AP14145. No drug-induced ventricular arrhythmias were observed up to the maximal dose of $20 \mathrm{mg} / \mathrm{kg}$ of AP14145.

\section{ERP and CV during regular pacing}

Atrial ERP was measured at a cycle length close to the fibrillation cycle length $(250 \mathrm{~ms})$ and at a cycle length closer to physiological sinus cycle length (400 ms). Figure 3 illustrates a profound ERP increase at both cycle lengths, $72 \pm 27 \mathrm{~ms}$ at $250 \mathrm{~ms}, \mathrm{p}=0.02$ and $83 \pm 15 \mathrm{~ms}$ at $400 \mathrm{~ms}$, $\mathrm{p}<0.001$. No rate-dependent effects on ERP were observed. At baseline, the CV varied from 77 to $73 \mathrm{~cm} / \mathrm{s}$ at a cycle length range from $225 \mathrm{~ms}$ to $450 \mathrm{~ms}$. AP14145 significantly reduced CV at most investigated cycle lengths $(\mathrm{p}<0.001)$. Moreover, a significant positive rate-dependent effect of AP14145 was present ( $<<0.001$ ), leading to about $20 \%$ slowing of the CV at the shortest cycle lengths. The wavelength at the cycle length of $400 \mathrm{~ms}$ increased by $5.7 \pm 1 \mathrm{~cm}$, $(\mathrm{p}<0.001)$. However, at a cycle length of $250 \mathrm{~ms}$ no change in wavelength was observed. 


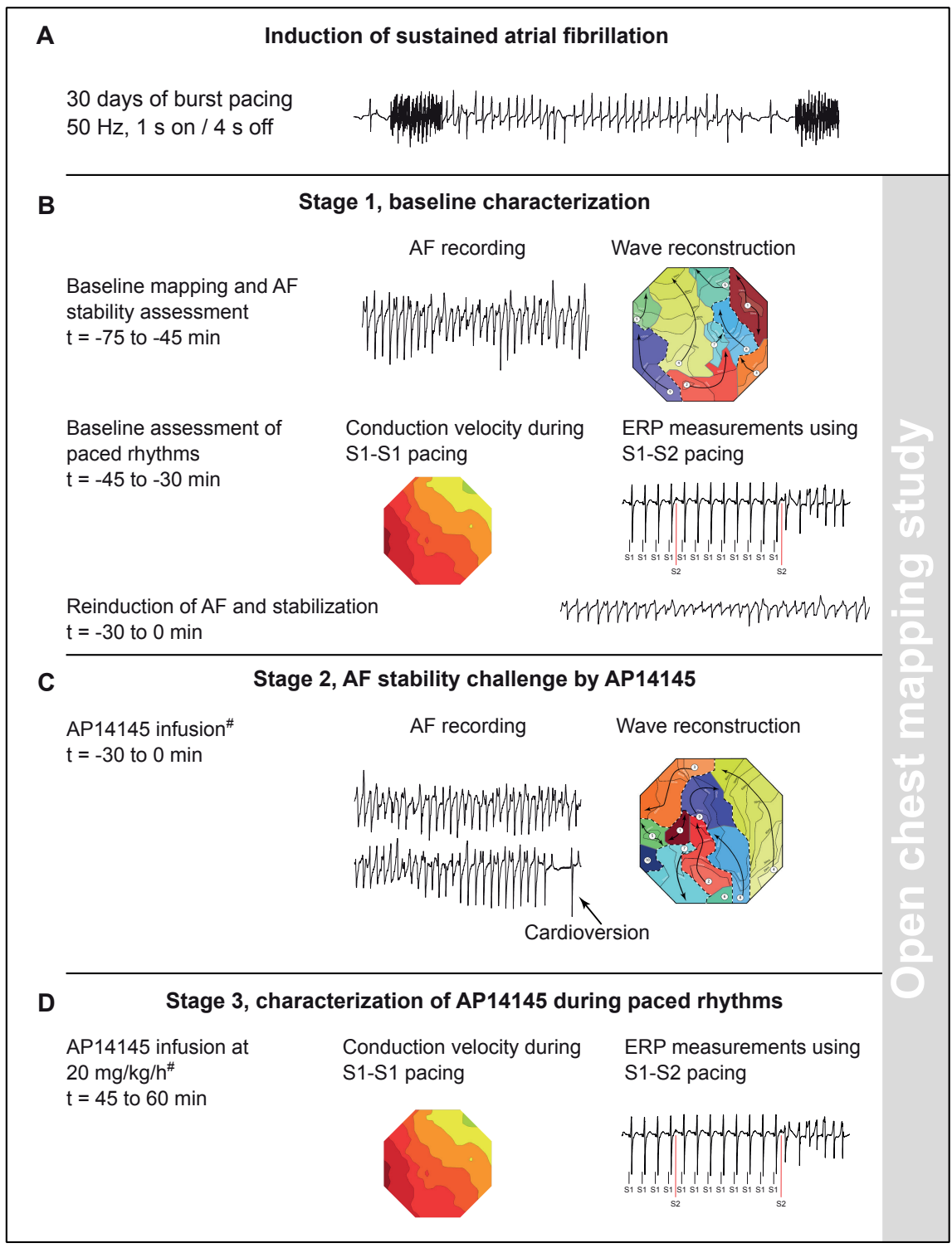

Figure 1. Graphical outline of the experimental design. (A) AF was induced and maintained by burst pacing for 30 days. At this point, 7 out of 8 goats were in sustained AF. The goat with unstable AF was excluded from the rest of the study. (B) In an open chest study, a baseline electrophysiological characterization was performed. Unipolar atrial electrograms were recorded during atrial pacing to assess ERP, conduction velocity (CV) and AF. (C) AF stability was challenged by the SK channel blocker AP14145. Unipolar atrial electrograms were recorded continuously during the intravenous administration of the drug. AF terminations were documented and if necessary, AF was reinduced. (D) ERP and CV were measured after 45 minutes of AP14145 administration. All ERP and CV measurements were performed during $20 \mathrm{mg} / \mathrm{kg} / \mathrm{h}$ AP14145 infusion.

" one goat was infused at a rate of $10 \mathrm{mg} / \mathrm{kg} / \mathrm{h}$ and 6 goats at a rate of $20 \mathrm{mg} / \mathrm{kg} / \mathrm{h}$. 
A

Goat 1

Goat 2

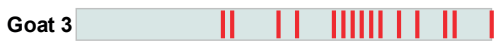

Goat $4 \quad||||||||||||||||||||||||||$

Goat 5

| ||||| | |||||

Goat $6 \quad$ || | |||||||||||| |||||| |||||||||||

Goat 7

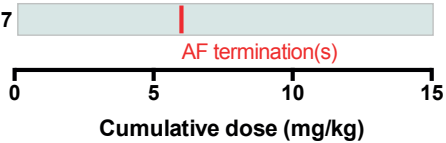

B

QRS duration
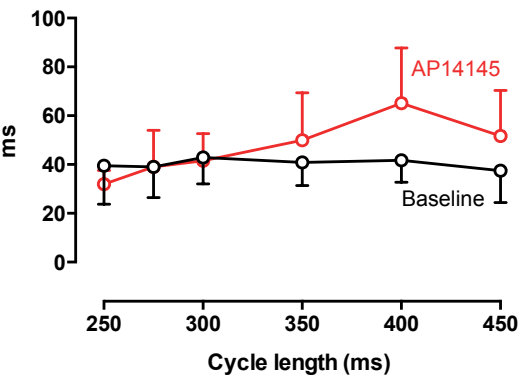

C LV systolic pressure

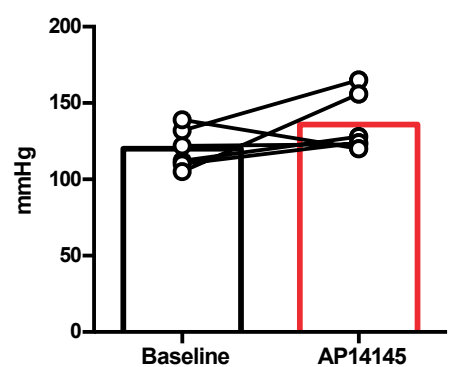

RR interval, sinus rhythm

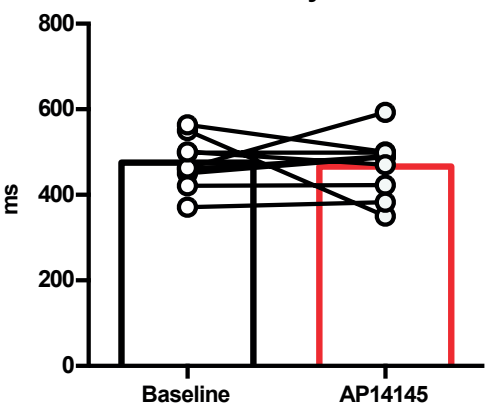

QT time

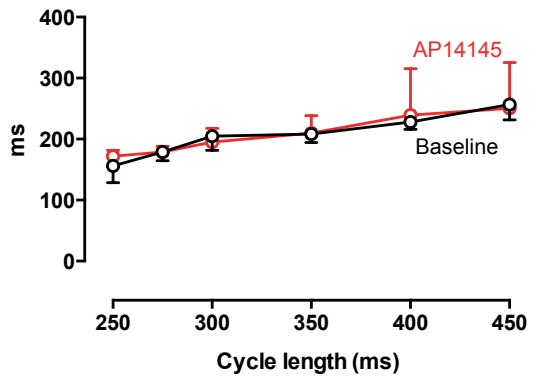

$\mathrm{LV} \mathrm{dP/dT} T_{\max }$

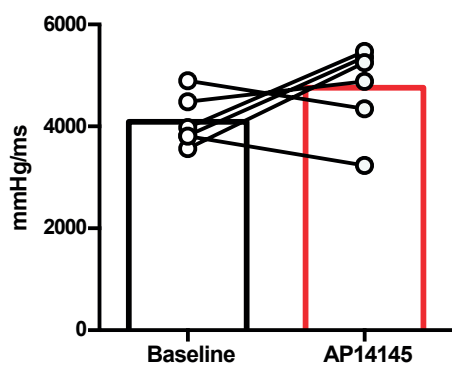

Figure 2. The effects of AP14145 infusion on AF stability and global ECG parameters. (A) The left panel depicts, per goat, the number of AF inductions (red lines) that were required to maintain AF during continuous AP14145 infusion. AF became highly unstable in 4 out of 5 the goats that cardioverted. The right panel illustrates the lack of effect of AP14145 (red bar) on the sinus cycle length. (B) The QRS durations and QT times were determined during pacing. AP14145 (red lines) did not significantly affect the QRS duration and QT time. Mean $\pm S D, n=6$. (C) At a cycle length of $400 \mathrm{~ms}, \mathrm{AP} 14145$ led to a trend towards higher systolic pressure and rate of pressure rise. Mean \pm SD, $\mathrm{n}=6$. 
A
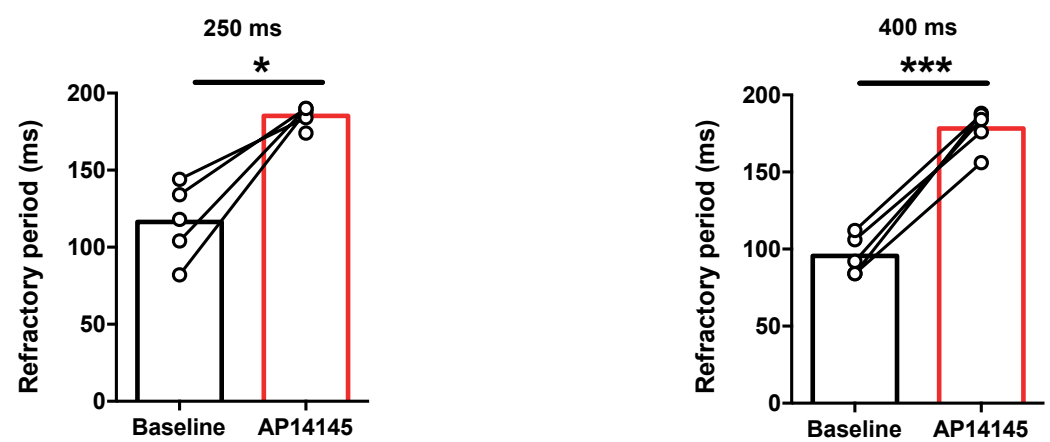

B
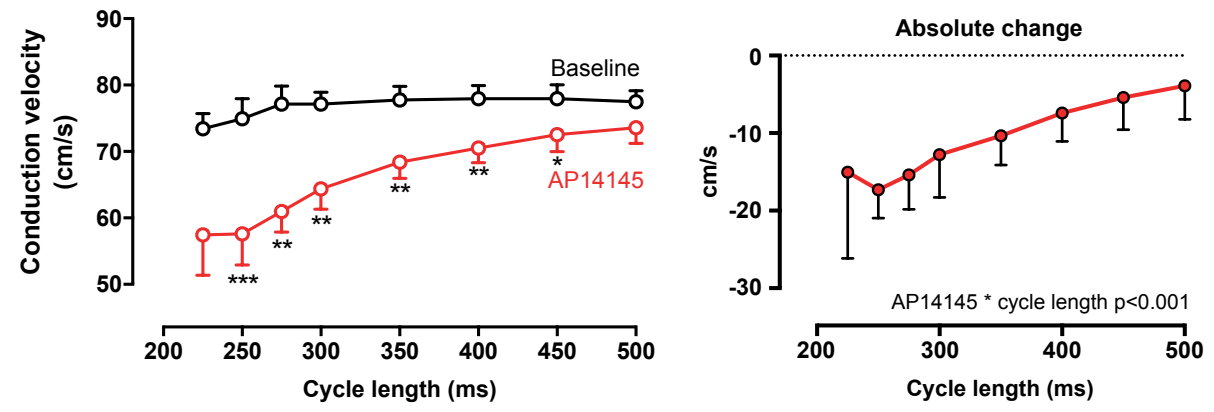

C
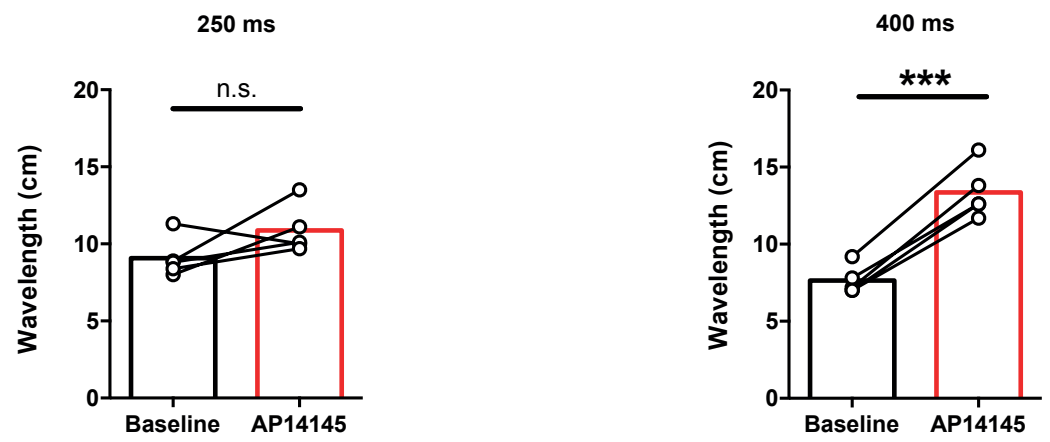

Figure 3. Electrophysiological effects of AP14145 during pacing. (A) Refractory period at a cycle length close to the AFCL (250 ms) and at a cycle length close to the sinus cycle length (400 ms). AP14145 significantly increased effective refractory periods at both cycle lengths. (B) Left atrial conduction velocity (CV) at a range of cycle lengths. AP14145 significantly reduced $\mathrm{CV}$ at most cycle lengths. From the absolute individual $\mathrm{CV}$ differences, it can be appreciated that AP14145 had a pronounced rate-dependent effect on CV. (C) The wavelength, calculated as a product of ERP and $\mathrm{CV}$. At a cycle length of $400 \mathrm{~ms}$, a significant increase was observed. However, no significant changes were observed at 250 ms. AP14145 vs. baseline ${ }^{*} \mathrm{p}<0.05,{ }^{* * *} \mathrm{p}<0.001, \mathrm{n}=6$. 


\section{The effect of AP14145 on AF properties}

Figure 4 presents the dose-dependent changes by AP14145 on AF. AF cycle length and the $5^{\text {th }}$ percentile of the AF cycle length presented a robust dose-dependent increase which persisted throughout all cumulative concentrations. At baseline (0 mg of AP14145), a right-to-left gradient of AF cycle length was present $(n=6, p<0.001)$. The gradient decreased somewhat at higher dosages of the drug but remained significantly different. Goats started to cardiovert (grey area in the figure) at moderate increases of AF cycle length. The goat that failed to cardiovert to sinus rhythm by AP14145 had an equivalent magnitude and steepness of AF cycle length increase in the LA compared to the goats that cardioverted but the steepness of AF cycle length increase in RA leveled off at a cumulative dose of $5 \mathrm{mg} / \mathrm{kg}$. The left atrial CV during baseline AF was lower compared to the CV measured during fast pacing, $58 \pm 4.6$ vs. $73 \pm 5.6 \mathrm{~cm} / \mathrm{s}(\mathrm{p}<0.01)$, respectively. CV did not change despite the robust increase in AF cycle length and cumulative dose of AP14145. In line with the stable CV and increasing AF cycle length, a consistent and significant increase in path length occurred in both atria with a trend towards stronger increases in the RA.

Next, we evaluated the type of conduction patterns (peripheral vs. breakthrough) and complexity of conduction in both atria (measured as the total number of waves). Surprisingly, the level of complexity showed only a trend towards fewer waves in the LA and no change was present in the RA. Increased number of breakthrough (BT) events or focal activity may be a source for the persistent high level of complexity. BT waves were therefore investigated for incidence and spatial distribution. Similar to the total number of waves, only a trend towards fewer BTs was found in the LA while no change was observed in the RA. BT waves occurred at all electrodes within the mapping area with certain sites of a higher BT activity. During AP14145 infusion, no sites of increased BT incidence were observed and the general spatial distribution of BTs remained stable. In the supplemental materials a more detailed report on the distribution of BTs is presented.

\section{A sudden transition in fibrillation pattern just prior to cardioversion}

Except for an AF cycle length and path length increase, no considerable changes in conduction properties were observed during the infusion of AP14145. We hypothesized that shortly before cardioversion, marked changes in fibrillation pattern occurred. We therefore analyzed the final 10 seconds prior to the (first) cardioversion. Figure 5 depicts four snapshots of conduction patterns during $\mathrm{AF}$ in a goat that terminated at a cumulative dose of $-5.4 \mathrm{mg}$. The complexity of conduction remained high (with 9-10 waves/cycle) till 10 seconds before AF termination. However, during the final cycles of AF the patterns rapidly reorganized, as illustrated by the two almost synchronized colliding waves in the lower right corner. 


\section{Left atrium}
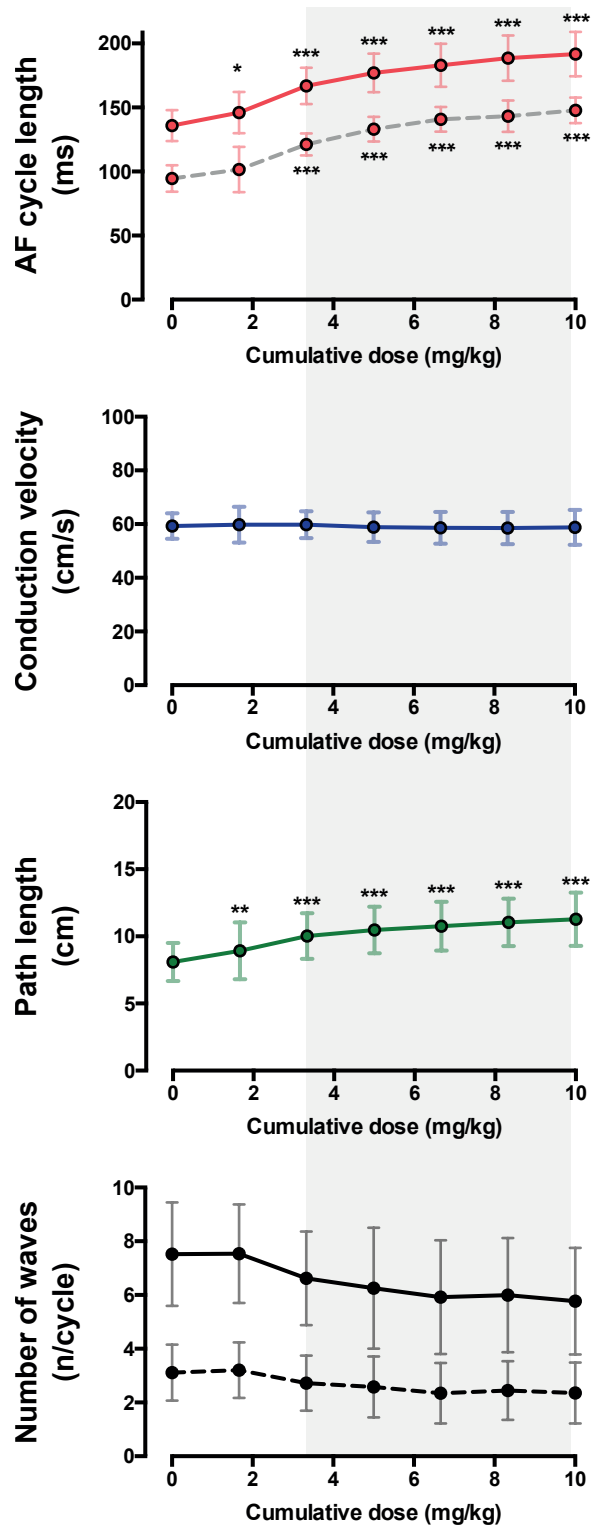

Right atrium
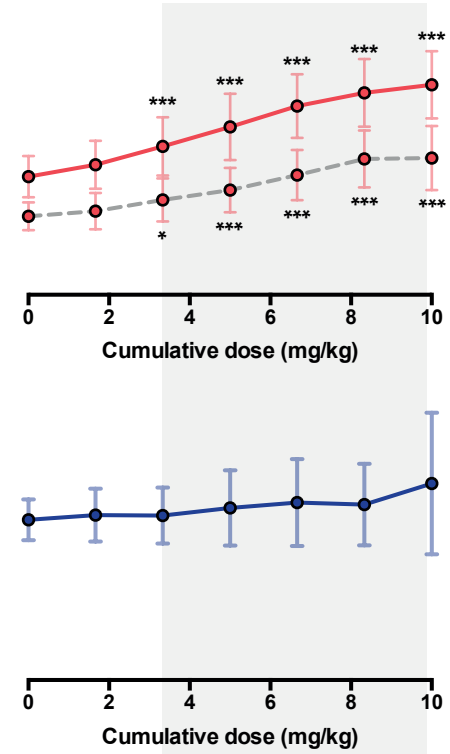

Figure 4. Electrophysiological parameters during AF at different cumulative doses of AP14145. AF cycle length (solid line) and the $5^{\text {th }}$ percentile (dashed line) of the AF cycle length increased in both atria in a dose-dependent manner. In contrast to the data during pacing, no changes in $\mathrm{CV}$ were observed in either atrium. A dose-dependent increase in path length (CV x AF cycle length) was observed in both atria. The total number of waves (solid line) and breakthroughs (dashed lines) exhibited a trend towards a reduction in the left atrium but not in the right atrium. AP14145 vs. baseline ${ }^{*} \mathrm{p}<0.05,{ }^{* *} \mathrm{p}<0.01,{ }^{* * *} \mathrm{p}<0.001, \mathrm{n}=7$. 


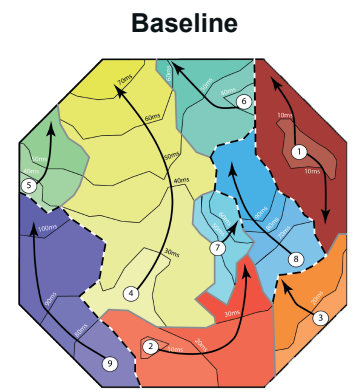

$5.4 \mathrm{mg}$ AP14145

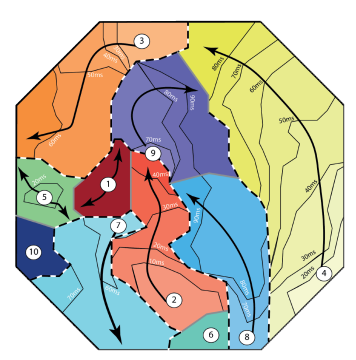

$5 \mathrm{mg}$ AP14145

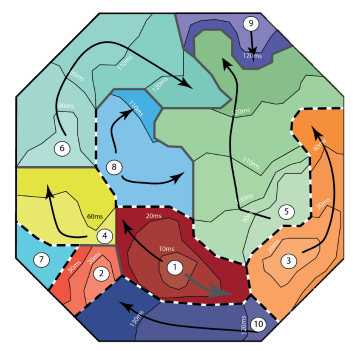

$5.4 \mathrm{mg} \mathrm{AP} 14145$ +9.5 seconds

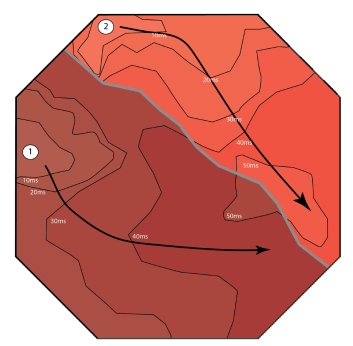

Order of wave appearences

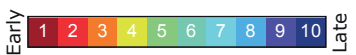

Block line

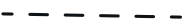

Collision/fusion line

Wave trajectory

Figure 5. Snapshots of conduction patterns of fibrillation waves in the left atrium in a time frame of 1 AF cycle. Upper left map, baseline. During baseline recording, there were on average 9.4 waves per AF cycle present in this goat. Wavefronts originating at the edge and breakthrough waves had a ratio of 1:1. Wavefronts often fused while at other sites lines of conduction block appeared, for example waves 1 to 4 . Upper right map, $5 \mathbf{~ m g} / \mathbf{k g}$. After 15 minutes of infusion, i.e., $5 \mathrm{mg} / \mathrm{kg}$, no significant changes in pattern complexity ( 8.4 waves/cycle) were observed. However, at this moment, the AF cycle length had increased from $139 \mathrm{~ms}$ to $183 \mathrm{~ms}$. Lower left map, $5.4 \mathbf{~ m g} / \mathbf{k g}$. One minute later, during the final $10 \mathrm{~s}$ before AF termination, 9.4 waves/cycle were present and the AF cycle length was around $180 \mathrm{~ms}$. Lower right map, the final fibrillation cycle. During the final cycle before termination, a rapid decline in number of waves occurred and the AF cycle length increased further to $198 \mathrm{~ms}$.

Figure 6 depicts the temporal changes in AF parameters (AF cycle length, CV, path length and number of waves) prior to cardioversion. To capture the dynamic behavior, we analyzed these parameters in windows of $500 \mathrm{~ms}$. Note that the cumulative dose differs between animals, as AF terminations occurred at different time points. At 10 seconds before AF termination, only the AF cycle length and path length were significantly increased while $\mathrm{CV}$ and waves were not significantly changed (Supplemental Table 1). Few changes were observed up to 2 seconds before $\mathrm{AF}$ termination but then rapid changes occurred, exhibited by a small increase in $\mathrm{CV}$ and a continuous further increase in AF cycle length and path length. The AF cycle length in the RA, which was initially shorter, underwent the steepest prolongation, exceeding the AFCL observed simultaneously in the LA. This coincided with a reduction in the number of waves per cycle and AF termination. The mechanistic interpretation of these changes is dependent on the existence of such events during stable (baseline) AF. Baseline AF was analyzed to identify 
large and abrupt fluctuations as seen just before AF termination. However, fluctuations of AF parameters to the level of AF termination were not observed at baseline, see the supplemental materials and Supplemental Figure 2 for more details.

\section{Wavefront stability during fast pacing}

As described above, complex fibrillation patterns persisted until just prior to AF termination. To explore wavefront stability as a source of complexity, we investigated wavefront propagation at short pacing cycle lengths $(225,250,275 \mathrm{~ms})$. Wave patterns were visually inspected and classified as uniformly propagating wavefronts, wavefronts with conduction block or failure to maintain 1:1 capture. A uniformly propagating wavefront was observed at all cycle lengths at baseline (Supplemental Table 2). In the presence of AP14145, the propagating wavefront at a pacing cycle length of $275 \mathrm{~ms}$ remained uniform (Figure 7A). However, a further reduction of the pacing cycle length led to more frequent conduction disorders and pacing failure. Figure $7 \mathrm{~B}$ illustrates a case of conduction block at a pacing cycle length of $225 \mathrm{~ms}$. Initially, only one entry point was present but the mapping area became activated from three sides with multiple block lines when AP14145 was present. To quantify more subtle changes in conduction, we investigated the spatial heterogeneity of conduction vectors by computing the circular variance of all conduction vectors at pacing cycle lengths of 225, 250 and 275 ms. Pacing at shorter cycle lengths led to more disperse conduction vectors (i.e., larger variance), both at baseline and in the presence of AP14145. Mixed model testing indicated an overall higher circular variance for AP14145 ( $\mathrm{p}=0.011$ ) but no individual cycle length showed significant difference. Similarly, the temporal variability of conduction direction (Figure 7C), measured as beat-tobeat angle differences, increased with shorter cycle lengths and was significantly increased by $\operatorname{AP} 14145(\mathrm{p}=0.026)$.

\section{DISCUSSION}

In this study we investigated electrophysiological effects of the novel SK channel inhibitor AP14145. When AP14145 was infused at a rate of $20 \mathrm{mg} / \mathrm{kg} / \mathrm{h}$, a high success rate $(5 / 6)$ of AF termination was achieved. This infusion regimen did not lead to changes in ventricular electrophysiology or left ventricular pump function. During pacing, a considerable slowing of atrial conduction was induced by AP14145, coinciding with a spatiotemporal instability of wavefront propagation. During AF, AP14145 induced a profound AF cycle length increase while conduction velocity was not affected. Surprisingly, no gradual reduction of AF complexity towards AF termination was observed. Instead, we report a sudden transition towards low complexity in the beats just prior to AF termination. From this study it remains unclear what triggers the sudden change in complexity, and further research is necessary to unravel this mechanism. Our data suggest that ERP and subsequent AF cycle length prolongation are the main antiarrhythmic mechanisms of AP14145. 
Left atrium

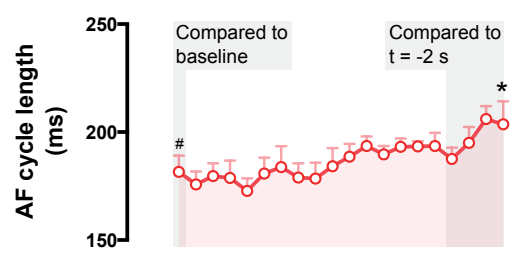

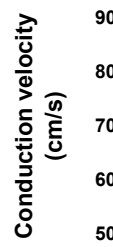
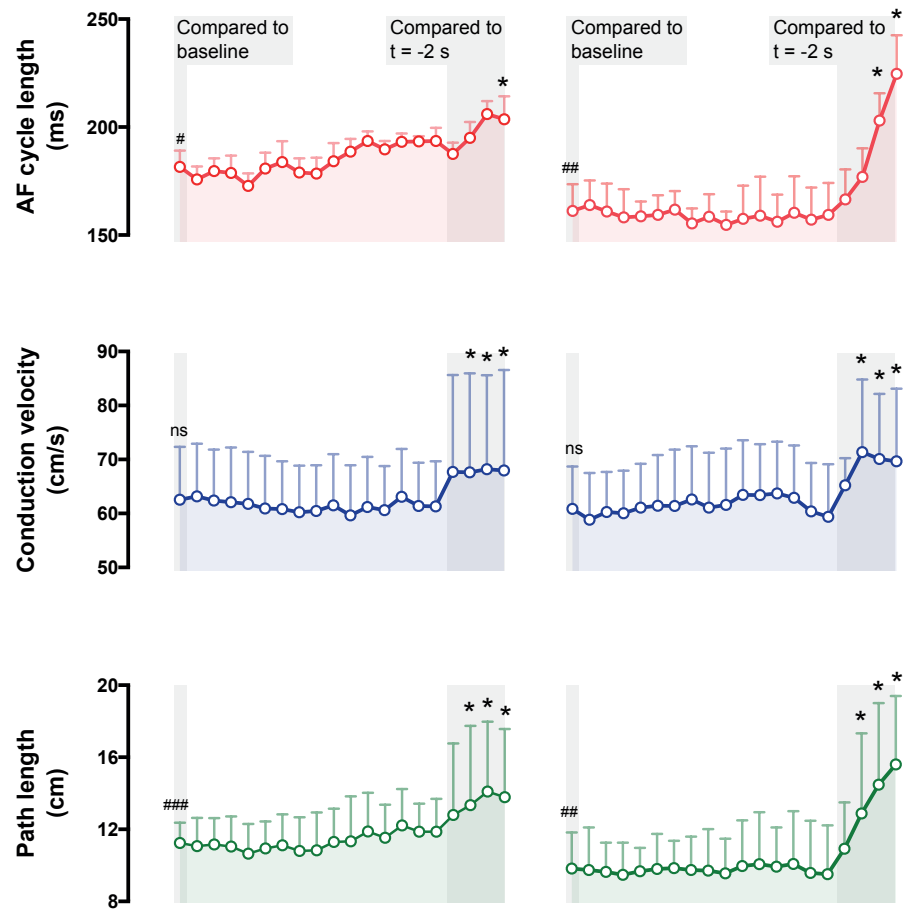

Right atrium
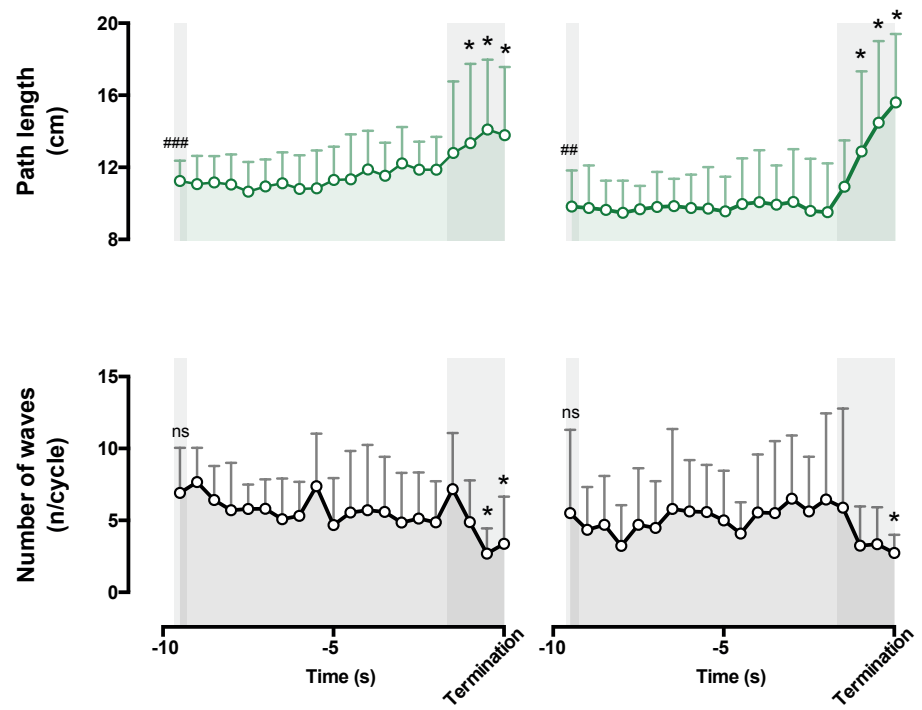

Figure 6. Temporal changes of AF properties within the last 10 seconds before AF termination. The data depict the first AF termination induced by AP14145 infusion. The right and left atrial data were recorded simultaneously. From top to bottom AF cycle length, conduction velocity, path length and the number of waves per AF cycle. The data were analyzed in non-overlapping windows of $500 \mathrm{~ms}$, which generally encompassed 2-3 AF cycles. In the first 8 seconds a slight trend towards a longer AF cycle and path length could be observed in the left atrium but not in the right atrium. In the final 2 seconds before termination, a small increase in conduction velocity in both atria was observed which was followed by a rapid increase of AF cycle length and path length. Somewhat later a decrease in the number of waves occurred. No significant differences between $-10 s$ to $-2 s$ were present but the differences appeared after the time point of -2 seconds. Data are presented as mean \pm SD. Versus baseline recording; ${ }^{\#} \mathrm{p}<0.05,{ }^{\# \#} \mathrm{p}<0.01$, $\# \# \mathrm{p}<0.001$. Versus time point $-2 \mathrm{~s} ;{ }^{*} \mathrm{p}<0.05, \mathrm{n}=5$. 


\section{A. Stability of wavefront propagation during fast pacing}
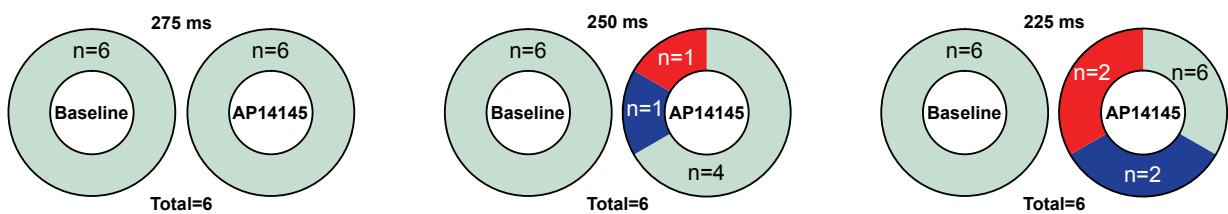

$\square$ Uniform wavefront

Propagation failure

No capture

\section{B. Changes of propagation direction during fast pacing}

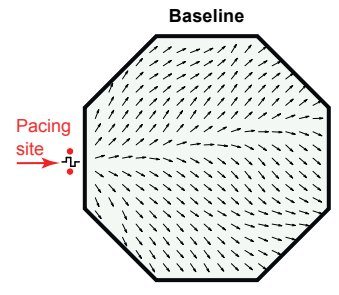

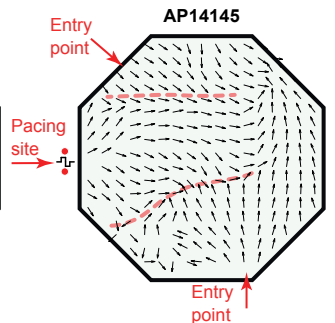

C. Beat to beat changes in conduction direction

Figure 7. (A) Donut charts illustrate the distribution of the type of wavefronts in response to fast S1-S1 pacing. Green slices represent homogenously and uniform conducting wavefronts, blue presents concave wavefronts or wavefronts with conduction block and red presents 1:1 conduction failure. (B) Two vector fields in the same goat at a pacing cycle length of $225 \mathrm{~ms}$. Bipolar pacing was performed on the left side of the array, electrodes are indicated by two red circles. At baseline, a homogenous wavefront can be appreciated. AP14145 led, in this case, to conduction block (red dashed line) at different sites and wavefront entry at different sites (red arrows), suggesting that the wavefront conducted along a tortuous path to enter the mapping array, despite the close proximity to the pacing site. (C) Temporal instability of conduction direction at three fast pacing rates. Variability of conduction direction increased when pacing cycle length was reduced and in the presence of AP14145. *vs. baseline, $\mathrm{p}<0.05, \mathrm{n}=6$.

\section{Safety and selectivity of the SK channel inhibitor AP14145}

Expression of SK channels has been reported for a variety of organs, including the heart and central nervous system (CNS). ${ }^{7}$ An SK channel inhibitor that does not pass the blood-brain barrier could potentially avoid CNS-related side effects. In the present work we studied the newly developed SK channel inhibitor AP14145, which has been presented as a possible atrialselective potassium channel inhibitor that exhibits an equipotent profile on different subtypes of SK channels in HEK cells. ${ }^{7}$ The administration of AP14145 did not lead to acute CNS effects in mice, ${ }^{7}$ but moderate tremors were observed in anesthetized pigs. ${ }^{9}$ In the current study we could not observe tremors as muscle relaxants were an integral component of the anesthesia. However, pilot pharmacokinetic studies in the awake goats revealed mild tremors after an intravenous bolus of $5 \mathrm{mg} / \mathrm{kg}$ of AP14145. 
SK channel selectivity of AP14145 has been evidenced by a minor inhibitory effect on hERG $\left(\mathrm{K}_{\mathrm{v}} 11.1\right)$ and lack of effect on $\mathrm{Na}_{\mathrm{v}} 1.5$ measured at a frequency of $1 \mathrm{~Hz} .{ }^{4}$ Atrial ERP prolongation without a prolongation of the ventricular ERP or QT interval has indeed been observed in different species with this small molecule compound. ${ }^{4,9,10}$ Safety profiling revealed no ventricular proarrhythmic effects. ${ }^{9}$ In line with these observations we did not observe QRS or QT prolongation nor ventricular arrhythmias, suggesting a high degree of atrial selectivity. Also, no hemodynamic instability was observed.

\section{SK channels, ERP and conduction velocity}

Early studies indicated that SK channels play a role in the late phase 3 repolarization of the cardiomyocyte. In a variety of species, and in this study, were demonstrated an action potential and ERP prolonging effects of SK channel inhibition. ${ }^{2-4}$ Treatment with SK channel inhibition results in shorter AF paroxysms and termination of sustained $\mathrm{AF}^{3,4} \mathrm{SK}$ channel inhibition was reported by Skibsbye et al. to also decrease $\mathrm{dV} / \mathrm{dt}_{\max }$ and action potential amplitude which was suggested to be caused by the observed minor depolarization of the resting membrane potential. $^{2}$ These observations were reported for two mechanistically different SK channel inhibitors, the negative allosteric modulator NS8593 and the direct pore inhibitor ICAGEN. Both drugs have a relatively high selectivity for SK2 and SK3, suggesting that SK inhibition generally leads to an indirect reduction of excitability which may be interpreted as a functional class I effect. ${ }^{2}$ However, Burashnikov et al. recently demonstrated that NS8593 also contains a direct and atrial-specific sodium current inhibiting property. ${ }^{11}$

In our study SK inhibition by AP14145 led to profound slowing of atrial CV and this was not coincided with QRS prolongation. AP14145 was reported not to affect $\mathrm{I}_{\mathrm{Na}}$ at $1 \mathrm{~Hz}^{4}$ but a direct $\mathrm{I}_{\mathrm{Na}}$ inhibition could have occurred at higher frequencies or at more depolarized holding potentials. From these experiments we cannot unravel the underlying mechanism for the functional class I effect we observed. Interestingly, CV during AF remained unaffected although AF cycle length prolonged profoundly. Drug-induced AF cycle length prolongation is associated with a widening of the temporal excitable gap. ${ }^{12}$ Therefore, it is likely that class III $\mathrm{AAD}$ treatment leads to longer sodium channel recovery times, resulting in an increase of $\mathrm{CV}$. Indeed, treatment of AF by the class III drug d-Sotalol increased both cycle length and CV during AF in the goat. ${ }^{12} \mathrm{CV}$ in the presence of AP14145 was slower than to be expected based on the AFCL prolongation, assuming that the widening of the temporal excitable gap applies to AP14145 too.

We argue that functionally a small class I effect was present during AF which may have contributed to the cardioversion potential of AP14145. The potential for a class I effect of SK inhibition is also further underscored by a series of experiments in Langendorff-perfused 
guinea pig hearts where it was shown that sub-efficacious doses of sodium channel blockers (flecainide and ranolazine) potentiate the antiarrhythmic effects of SK channel inhibition. ${ }^{6}$ Possibly, such synergistic potential may apply to AP14145 as well.

\section{AF cycle length prolongation is the first step to AP14145-induced AF termination}

In a tachypaced pig model of AF, Diness et al. demonstrated a high potency of AP14145 to treat $\mathrm{AF}^{4}$ This was demonstrated by the ability of AP14145 to terminate AF at a stage of remodeling where clinical dosages of vernakalant could no longer terminate $A F$. In the present study, AP14145 terminated persistent $\mathrm{AF}$ in $83 \%$ of the goats within $10-30$ minutes at an infusion rate of $20 \mathrm{mg} / \mathrm{kg} / \mathrm{h}$.

Short AF cycle lengths and atrial fibrosis are thought to be one of the main contributors to conduction block, complex fibrillation patterns and AF stability. ${ }^{13}$ Rotor dynamics and arrhythmia stability are also modulated by the cycle length. ${ }^{14}$ This was also reported by Schuessler et al., who demonstrated in canine atria that the shortening of the AF cycle length alone is sufficient to increase AF stability, wave-break and fibrillation complexity. ${ }^{15}$ The most consistent effect of antiarrhythmic drugs, despite their wide variety of targets, is the dosedependent increase of the AF cycle length. ${ }^{16}$ In this study AP14145 also strongly increased ERP that was translated to a substantial increase of AF cycle length and path length. We consider these effects as the main mechanism for antiarrhythmic effect of AP14145.

Using contact mapping, Wang and Nattel showed that a drug-induced prolongation of AF cycle length was indeed associated with fewer propagating wavefronts. ${ }^{17}$ Remarkably, in the current study complex conduction patterns persisted until the final seconds before AF termination. Our findings may suggest that AP14145 affects fibrillatory conduction in such a way that it becomes more prone to termination without decreasing complexity. The process of termination itself is characterized by the observed rapid change in the whole electrophysiological profile (i.e., cycle length, $\mathrm{CV}$, path length and ultimately complexity of propagation patterns). From this study it remains unclear what triggers the sudden change in behavior, and further research is necessary to unravel this mechanism.

\section{No organization of conduction patterns occurred despite a significant prolongation in AF cycle length}

The combination of AF cycle length prolongation and persistence of complex patterns is an intriguing observation. It is conceivable that SK channel inhibition by AP14145 in our study produced both antiarrhythmic effects (APD prolongation and reduced excitability) and some proarrhythmic effects. Although this and other studies found inhibition of SK channels to 
be antiarrhythmic, ${ }^{3,4}$ an important biological role for SK channels has been proposed as it contributes to the repolarization reserve ${ }^{18}$ and inhibition may therefore have proarrhythmic effects.

One proarrhythmic mechanism of SK channel block is thought to be based on the tight link between free cytosolic $\mathrm{Ca}^{2+}$ concentration and the SK channel conductance. SK channels would thereby counteract spontaneous $\mathrm{Ca}^{2+}$ release events from the sarcoplasmic reticulum and suppress focal activity. ${ }^{19}$ Yet, investigations of SK channel inhibition in models with ventricular substrates for arrhythmias, ischemia and pacing-induced heart failure demonstrated fewer premature ventricular contractions and spontaneous episodes of ventricular tachycardia or fibrillation. ${ }^{20}$ In the context of AF, more frequent focal activity, observed as breakthrough patterns, would obviously contribute to a higher complexity. However, our data did not give an indication of an increased incidence of breakthrough patterns nor an altered distribution of breakthrough sites.

A proarrhythmic mechanism at tissue level was observed for SK channel inhibition in an optical mapping study in canine left atria. The authors showed higher degrees of action potential dispersion, alternans and the propensity towards wave-break during S1-S1 pacing when SK current was inhibited by apamin. ${ }^{21}$ Similar effects were also observed in the rabbit ventricle under hypokalemic conditions, leading to more wavefronts (phase singularities) during ventricular fibrillation. ${ }^{18}$ In our study, a higher spatiotemporal instability and propensity towards wave-break and propagation failure during fast pacing rates were observed in the presence of AP14145. The direct mechanism for this instability of wavefront propagation could not be investigated in this study. It is conceivable that the mechanism leading towards wavefront instability contributed to the maintained AF complexity and may play a critical role in the sudden transition towards reorganization and $\mathrm{AF}$ termination.

\section{LIMITATIONS}

This study is limited by a number of aspects. First, SK channel expression levels in the goat are unknown. However, SK channel expression is reported for a wide variety of species, e.g., rat, ${ }^{20}$ dogs, ${ }^{3}$ pigs,${ }^{4}$ and humans. ${ }^{2}$ Considering this and the atrial-specific electrophysiological effects, we believe it is highly likely that our observations are caused by atrial SK channel inhibition. Second, the in vivo approach of high-density mapping did not allow for action potential recording. This limits our understanding on the contribution of the class I effect on the ERP prolongation and during AF itself. Third, the sample size was relatively small. This may have affected the statistical power to detect differences for some parameters. Fourth, mapping the atria with the two mapping arrays provides coverage of approximately $50 \%$ of the atrial surface. 
The data are discussed on the assumption that mapping of the free walls is representative for AF behavior over the entire atria. However, if AF mechanisms that could drive AF and maintain complexity occurred outside of the field of view, they could have remained undetected. Finally, no pharmacokinetic data were included in this study. The results can therefore not be directly correlated to plasma levels.

\section{CONCLUSION}

AP14145 provided a successful therapy for the termination of persistent AF in the goat. Its antiarrhythmic mechanism during $\mathrm{AF}$ is mainly based on ERP and AF cycle length prolongation. A class I effect was present in the atria, but not in the ventricles, during pacing and to a smaller extent also during AF. These electrophysiological effects did not lead to a gradual but a sudden organization of conduction. The mechanisms of these sudden changes need to be elucidated in further studies. 


\section{REFERENCES}

1. Brandes A, Crijns HJGM, Rienstra M, Kirchhof P, Grove EL, Pedersen KB, et al. Cardioversion of atrial fibrillation and atrial flutter revisited: current evidence and practical guidance for a common procedure. Europace 2020;22:1149-1161.

2. Skibsbye L, Poulet C, Diness JG, Bentzen BH, Yuan L, Kappert U, et al. Small-conductance calcium-activated potassium (SK) channels contribute to action potential repolarization in human atria. Cardiovasc Res 2014;103:156-167.

3. Qi X, Diness JG, Brundel BJJ, Zhou X, Naud P, Wu C, et al. Role of small-conductance calciumactivated potassium channels in atrial electrophysiology and fibrillation in the dog. Circulation 2014;129:430-440.

4. Diness JG, Skibsbye L, Simó-Vicens R, Santos JL, Lundegaard P, Citerni C, et al. Termination of Vernakalant-Resistant Atrial Fibrillation by Inhibition of Small-Conductance $\mathrm{Ca}^{2+}$-Activated $\mathrm{K}^{+}$ Channels in Pigs. Circ Arrhythm Electrophysiol 2017;10:e005125.

5. Skibsbye L, Bengaard AK, Uldum-Nielsen AM, Boddum K, Christ T, Jespersen T. Inhibition of Small Conductance Calcium-Activated Potassium (SK) Channels Prevents Arrhythmias in Rat Atria During ß-Adrenergic and Muscarinic Receptor Activation. Front Physiol 2018;9:1453.

6. Kirchhoff JE, Diness JG, Sheykhzade M, Grunnet M, Jespersen T. Synergistic antiarrhythmic effect of combining inhibition of $\mathrm{Ca}^{2+}$-activated $\mathrm{K}^{+}(\mathrm{SK})$ channels and voltage-gated $\mathrm{Na}^{+}$channels in an isolated heart model of atrial fibrillation. Heart Rhythm 2015;12:409-418.

7. Simó-Vicens R, Kirchhoff JE, Dolce B, Abildgaard L, Speerschneider T, Sørensen US, et al. A new negative allosteric modulator, AP14145, for the study of small conductance calcium-activated potassium $\left(\mathrm{K}_{\mathrm{Ca}} 2\right)$ channels. Br J Pharmacol 2017;174:4396-4408.

8. Zeemering S, Maesen B, Nijs J, Lau DH, Granier M, Verheule S, et al. Automated quantification of atrial fibrillation complexity by probabilistic electrogram analysis and fibrillation wave reconstruction. Conf Proc IEEE Eng Med Biol Soc 2012;2012:6357-6360.

9. Lubberding AF, Sattler SM, Grunnet M, Sørensen US, Tfelt-Hansen J, Jespersen T. Arrhythmia development during inhibition of small-conductance calcium-activated potassium channels in acute myocardial infarction in a porcine model. Europace 2019;21:1584-1593.

10. Kirchhoff JE, Skarsfeldt MA, Muthukumarasamy KM, Simó-Vicens R, Bomholtz SH, Abildgaard L, et al. The $\mathrm{K}_{\mathrm{Ca}} 2$ Channel Inhibitor AP14145, But Not Dofetilide or Ondansetron, Provides Functional Atrial Selectivity in Guinea Pig Hearts. Front Pharmacol 2019;10:668.

11. Burashnikov A, Barajas-Martinez H, Hu D, Robinson VM, Grunnet M, Antzelevitch C. The Small Conductance Calcium-Activated Potassium Channel Inhibitors NS8593 and UCL1684 Prevent the Development of Atrial Fibrillation Through Atrial-Selective Inhibition of Sodium Channel Activity. J Cardiovasc Pharmacol 2020;76:164-172.

12. Wijffels MC, Dorland R, Mast F, Allessie MA. Widening of the excitable gap during pharmacological cardioversion of atrial fibrillation in the goat: effects of cibenzoline, hydroquinidine, flecainide, and d-sotalol. Circulation 2000;102:260-267.

13. Haïssaguerre M, Lim K-T, Jacquemet V, Rotter M, Dang L, Hocini M, et al. Atrial fibrillatory cycle length: computer simulation and potential clinical importance. Europace 2007;9 Suppl 6:vi64-70. 
14. Laksman Z, Wauchop M, Lin E, Protze S, Lee J, Yang W, et al. Modeling Atrial Fibrillation using Human Embryonic Stem Cell-Derived Atrial Tissue. Sci Rep 2017;7:1042.

15. Schuessler RB, Grayson TM, Bromberg BI, Cox JL, Boineau JP. Cholinergically mediated tachyarrhythmias induced by a single extrastimulus in the isolated canine right atrium. Circ Res 1992;71:1254-1267.

16. Workman AJ, Smith GL, Rankin AC. Mechanisms of termination and prevention of atrial fibrillation by drug therapy. Pharmacol Ther 2011;131:221-241.

17. Wang Z, Fermini B, Nattel S. Mechanism of flecainide's rate-dependent actions on action potential duration in canine atrial tissue. J Pharmacol Exp Ther 1993;267:575-581.

18. Chan YH, Tsai WC, Ko JS, Yin D, Chang PC, Rubart M, et al. Small-Conductance CalciumActivated Potassium Current Is Activated During Hypokalemia and Masks Short-Term Cardiac Memory Induced by Ventricular Pacing. Circulation 2015;132:1377-1386.

19. Terentyev D, Rochira JA, Terentyeva R, Roder K, Koren G, Li W. Sarcoplasmic reticulum $\mathrm{Ca}^{2+}$ release is both necessary and sufficient for SK channel activation in ventricular myocytes. AJP: Heart and Circulatory Physiology 2014;306:H738-H746.

20. Gui L, Bao Z, Jia Y, Qin X, Cheng ZJ, Zhu J, et al. Ventricular tachyarrhythmias in rats with acute myocardial infarction involves activation of small-conductance $\mathrm{Ca}^{2+}$-activated $\mathrm{K}^{+}$channels. AJP: Heart and Circulatory Physiology 2013;304:H118-H1130.

21. Hsueh CH, Chang PC, Hsieh YC, Reher T, Chen PS, Lin SF. Proarrhythmic effect of blocking the small conductance calcium activated potassium channel in isolated canine left atrium. Heart Rhythm 2013;10:891-898. 


\section{SUPPLEMENTAL MATERIALS}

\section{Breakthrough wave analysis}

The incidence and distribution of breakthrough patterns as a cause of the maintained complex patterns were analyzed to investigate the role of breakthroughs on maintained AF complexity after AP14145 infusion. For this purpose, we used AF recordings at baseline and at the cumulative doses of 5 and $10 \mathrm{mg} / \mathrm{kg}$. Supplemental Figure 1 depicts heatmaps of the relative number of breakthroughs at each electrode. The Pearson's correlation coefficient between baseline and drug conditions was quantified to illustrate the stability of the breakthrough distribution. The correlation coefficients between baseline and drug conditions indicated only small differences in breakthrough distributions. The ratio between breakthroughs and the total number of waves was quantified to identify a possible change in the number of waves with a focal origin as source for waves. Only in the left atrium at a dose of $10 \mathrm{mg} / \mathrm{kg}$ a slight reduction occurred compared to baseline $(0.53 \pm 0.04$ vs. $0.49 \pm 0.05, \mathrm{p}=0.004)$, suggesting a lower contribution of breakthroughs.

\section{Perturbations of AF parameters during baseline AF}

Sudden and significant changes in AF cycle length, conduction velocity, path length and the number of waves per cycle were observed just prior to AF termination. The mechanistic interpretation of these changes is dependent on the existence of such events during stable (baseline) AF. Considering the AF termination profiles, we regarded the AF cycle length prolongation to be the first and strongest effect. Baseline AF was analyzed to identify the time point with the longest $\mathrm{AF}$ cycle length in a 60 -second recording. For all five goats that cardioverted, an AF property time plot was created with 10 seconds before and after the occurrence of the longest cycle length. Despite the alignment to the maximal AF cycle length, no considerable changes in AF parameters were observed. 

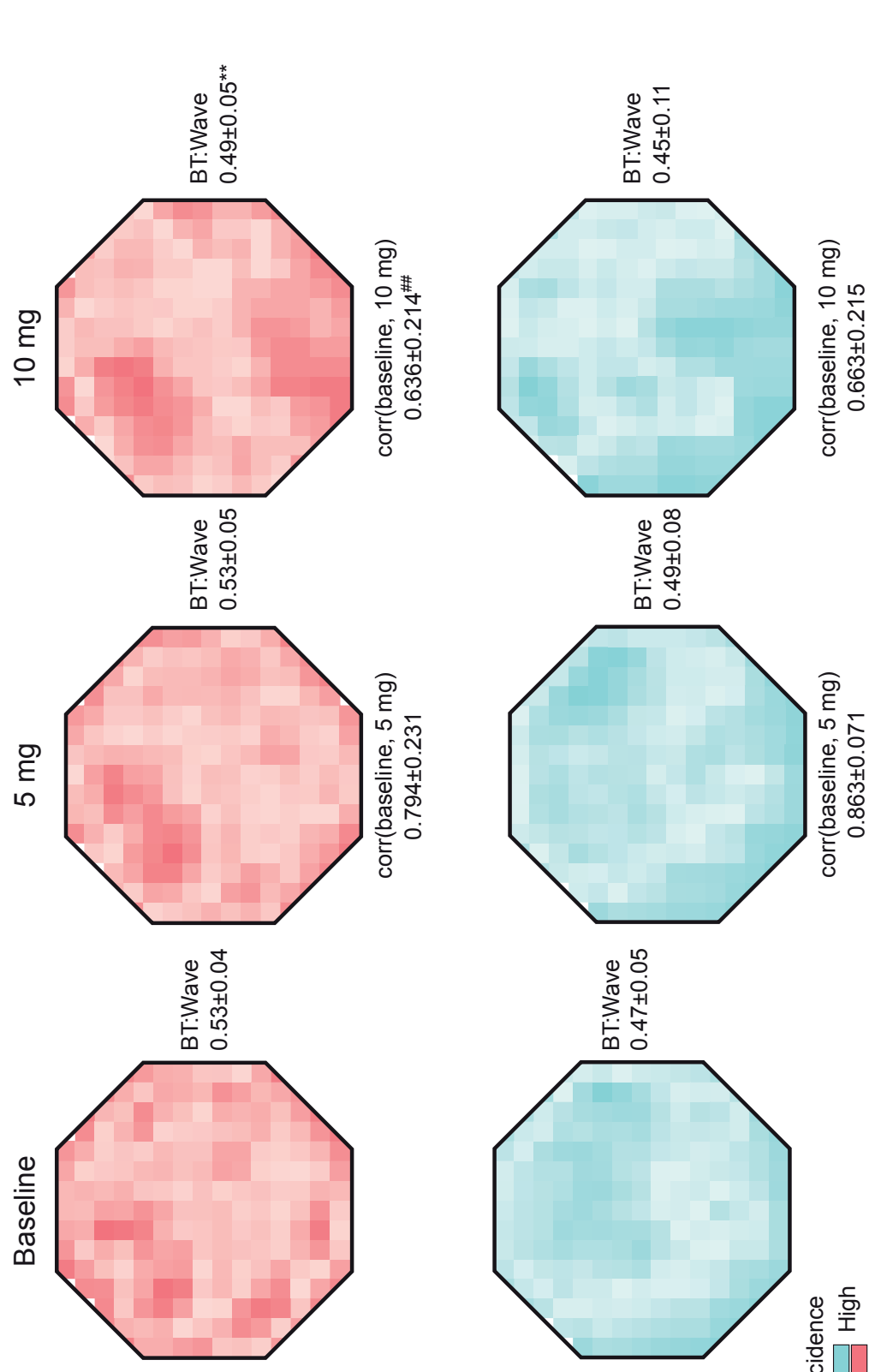

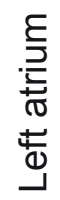
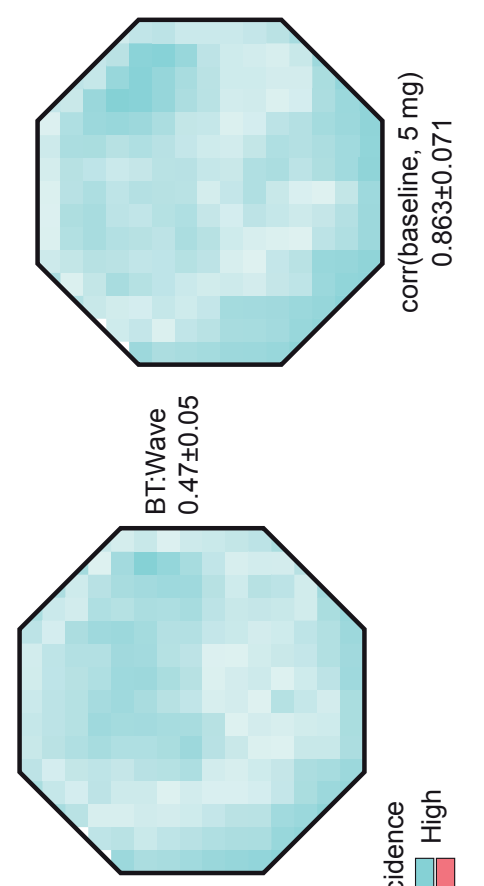

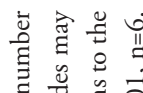

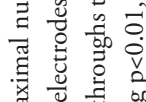

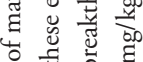

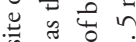

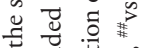

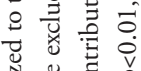

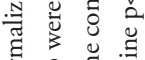

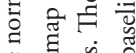

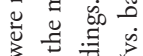

出 현

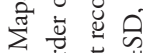

i่

$\exists$

$\sum_{0} \underbrace{0}_{0}$

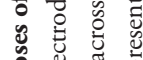

它造

节 芦芯

密

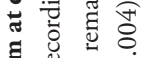

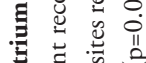

吾离

总㟽要

들 홍

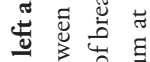

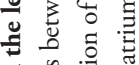

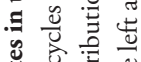

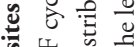

崖寻

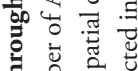

苛芯芯

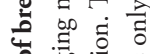

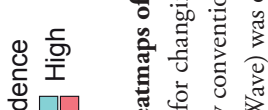

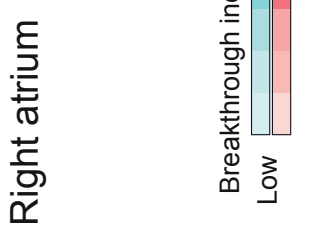

范客

-

웡

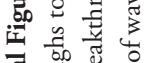

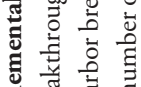

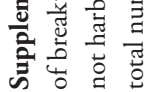


Left Atrium
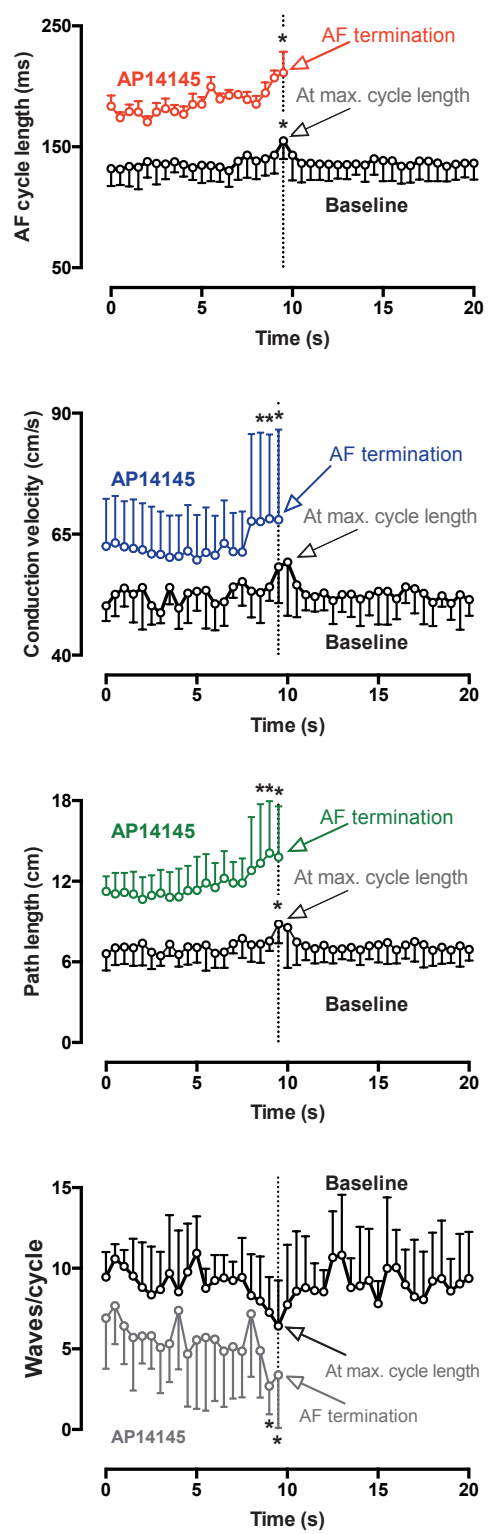

Right Atrium
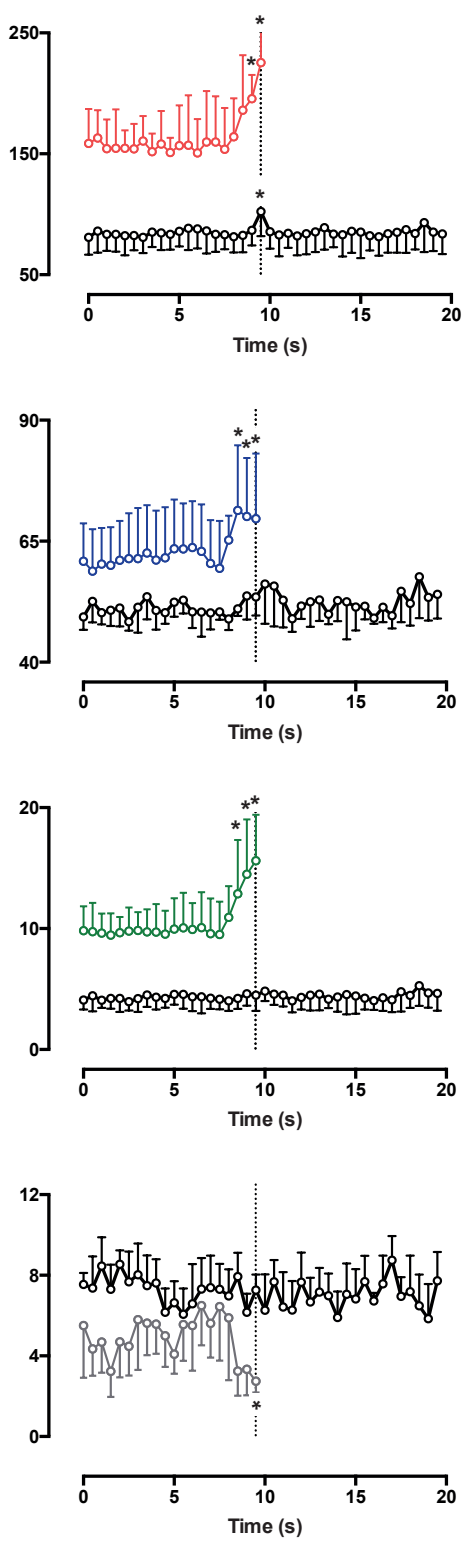

Supplemental Figure 2. Temporal behavior of AF parameters during baseline AF and AP14145-induced AF termination. Baseline data are presented as black circles (mean) and error bars (SD). For AP14145-induced AF termination the AF cycle length was plotted in red, conduction velocity in blue, path length in green and the number of waves per cycle in grey. As in Figure 6, we analyzed the mapping data in non-overlapping bins of $500 \mathrm{~ms}$. The baseline recording is presented as 10 seconds before and the 10 seconds after the point of highest AF cycle length. ${ }^{*} \mathrm{p}<0.05$. 
Supplemental Table 1. Changes in several electrophysiological properties from baseline to 10 seconds before AF termination. Parameters were determined over a $500 \mathrm{~ms}$ window. Mean \pm SD, $10 \mathrm{~s}$ before termination vs. baseline ${ }^{* *} \mathrm{p}<0.01,{ }^{* * *} \mathrm{p}<0.001, \mathrm{n}=5$.

\begin{tabular}{llcc}
\hline Parameter & Time point & Left atrium & Right atrium \\
\hline AF cycle length & Baseline & $134 \pm 11$ & $92 \pm 19$ \\
$(\mathrm{~ms})$ & $\mathrm{T}=-10 \mathrm{~s}$ & $183 \pm 19^{* *}$ & $158 \pm 28^{* *}$ \\
& Absolute difference & $49 \pm 25$ & $62.53 \pm 9.7$ \\
$\mathrm{CV}$ & Baseline & $59.22 \pm 7.5$ & $55.82 \pm 7.2$ \\
$(\mathrm{~cm} / \mathrm{s})$ & $\mathrm{T}=-10 \mathrm{~s}$ & $62.53 \pm 9.7$ & $60.85 \pm 7.8$ \\
& Absolute difference & $3.32 \pm 7.9$ & $5.03 \pm 9.5$ \\
Path length & Baseline & $8.04 \pm 1.5$ & $5.2 \pm 1.4$ \\
$(\mathrm{~cm})$ & $\mathrm{T}=-10 \mathrm{~s}$ & $11.25 \pm 1.1 * * *$ & $9.82 \pm 2^{* *}$ \\
& Absolute difference & $3.21 \pm 0.6$ & $4.62 \pm 2.1$ \\
Waves & Baseline & $8.45 \pm 4$ & $6.62 \pm 5$ \\
$(1 /$ cycle $)$ & $\mathrm{T}=-10 \mathrm{~s}$ & $6.91 \pm 3.1$ & $5.51 \pm 5.8$ \\
& Absolute difference & $-1.54 \pm 6.3$ & $-1.11 \pm 1.6$ \\
\hline
\end{tabular}

Supplemental Table 2. Summary of the visually scored wavefront patterns at 3 different short pacing cycle lengths for all goats. Wavefronts were classified into 3 types: uniform, block line and no or unstable capture. BT = breakthrough.

\begin{tabular}{|c|c|c|c|c|c|c|}
\hline \multirow[t]{2}{*}{ Goat ID } & \multicolumn{2}{|c|}{ BCL: $275 \mathrm{~ms}$} & \multicolumn{2}{|c|}{ BCL: $250 \mathrm{~ms}$} & \multicolumn{2}{|c|}{ BCL: $225 \mathrm{~ms}$} \\
\hline & Baseline & AP14145 & Baseline & AP14145 & Baseline & AP14145 \\
\hline 18589 & Uniform & Uniform & Uniform & Uniform & Uniform & Uniform \\
\hline 19226 & Uniform & Uniform & Uniform & Uniform & Uniform & $\begin{array}{c}\text { Concave } \\
\text { wavefront }\end{array}$ \\
\hline 20072 & Uniform & Uniform & Uniform & Uniform & Uniform & Unstable capture \\
\hline 20771 & Uniform & Uniform & Uniform & $\begin{array}{l}\text { One block line } \\
\text { followed by a BT }\end{array}$ & Uniform & $\begin{array}{l}\text { Conduction block } \\
\text { at multiple sites }\end{array}$ \\
\hline 20797 & Uniform & Uniform & Uniform & Uniform & Uniform & Uniform \\
\hline 21097 & Uniform & Uniform & Uniform & Unstable capture & Uniform & Unstable capture \\
\hline
\end{tabular}




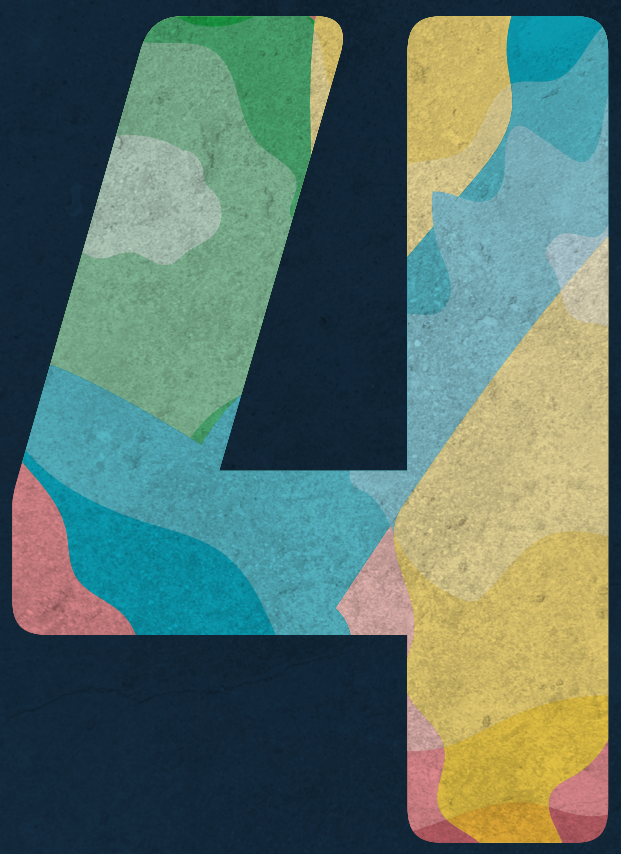




\section{Shared mechanisms of cardioversion of atrial fibrillation in the goat: acceleration of atrial conduction and inter-atrial synchronization}

Vladimír Sobota*, Arne van Hunnik*, Stef Zeemering, Giulia Gatta, Dragan Opacic, Billy Scaf, Elisa D’Alessandro, Karel Oyaert, Marion Kuiper, Rachelle Peeters, Jonas G. Dines, Ulrik S. Sørensen, James Milnes, Marcel A. G. van der Heyden, Ulrich Schotten and Sander Verheule

* the authors contributed equally

In preparation 


\section{ABSTRACT}

Background: The mechanisms leading to the conversion of atrial fibrillation (AF) to sinus rhythm (SR) are still poorly understood. This study investigates electrophysiological parameters and conduction patterns acquired during the last seconds before pharmacological and spontaneous termination of AF.

Methods: A retrospective evaluation of 6 independent studies in goats ( $n=33)$ was performed. AF was induced and maintained with an implanted pacemaker for 3-4 weeks in 29 animals, the remaining 4 animals were kept in SR. During an open chest experiment, unipolar electrograms were acquired with a 249-electrode array from each atrium. Pharmacological termination of AF was attempted with class III antiarrhythmic drugs (AADs) AP14145 (n=5), PA-6 (n=7), XAF-1407 ( $n=9)$ and class I AAD vernakalant $(n=8)$. In the SR animals AF was acutely induced. Baseline AF and up to 3 recordings of the last 10 seconds preceding AF termination were acquired in each animal. Intervals with continuous and periodic activity were determined in the recordings, representing phases of higher and lower AF complexity, respectively. AF cycle length (AFCL), conduction velocity and path length were determined for each interval.

Results: In total, 85 recordings of AF termination were obtained. Cardioversion of AF was preceded by sudden increase in AF organization, characterized by an interval of periodic activity. This interval was associated with increases in AFCL (LA: $163 \pm 37$ vs. $204 \pm 50 \mathrm{~ms}, \mathrm{p}<0.0001$; RA: $146 \pm 44$ vs. $207 \pm 49 \mathrm{~ms}, \mathrm{p}<0.0001$ ), and conduction velocity (LA: $77 \pm 15 \mathrm{vs} .96 \pm 25 \mathrm{~cm} / \mathrm{s}$, $\mathrm{p}<0.0001$; RA: $83 \pm 20$ vs. $103 \pm 24 \mathrm{~cm} / \mathrm{s}, \mathrm{p}<0.0001$ ), resulting in prolongation of path length (LA: $12.2 \pm 2.7$ vs. $19.3 \pm 6.3 \mathrm{~cm}, \mathrm{p}<0.0001$; RA: $12.0 \pm 4.1$ vs. $21.2 \pm 6.4 \mathrm{~cm}, \mathrm{p}<0.0001$ ). In $92 \%$ of cardioversions the conduction patterns suggested a pivotal role of Bachmann's bundle.

Conclusion: AF termination is preceded by an increased organization of fibrillatory conduction, associated with abrupt prolongation of AFCL and acceleration of conduction velocity, resulting in prolongation of path length. 


\section{INTRODUCTION}

Mechanisms underlying perpetuation of atrial fibrillation (AF) have been extensively investigated over the last decades. Several concepts have been proposed as important contributors, including anatomical and functional reentry, ${ }^{1-4}$ ectopic activity, ${ }^{5,6}$ multiple wavelets $^{7,8}$ and endo-epicardial dissociation. ${ }^{9-11}$ Yet, no consensus has been reached regarding the relative importance with which these mechanisms contribute to AF maintenance in humans and animal models, making the topic widely debated. ${ }^{12,13}$ Also, the mechanisms of conversion of AF to sinus rhythm (SR) are not well understood. During this transition, AFdriving mechanisms disappear or become insufficient to maintain the arrhythmia. Performing electrical mapping during the last moments preceding cardioversion of AF might thus identify critical events that determine AF termination.

Several studies have investigated the transition of persistent AF to SR under the influence of various antiarrhythmic drugs (AADs). ${ }^{14-21}$ Organization of fibrillation pattern and a reduction in the number of wavefronts has been shown to precede AF termination after administration of class III AADs XAF-1407 (chapter 2), AP14145 (chapter 3) and pentamidine-analogue 6 (PA-6). ${ }^{19}$ However, to our knowledge, there have been only few studies that mapped the actual conversion of AF to SR. Mapping studies suggest that both conduction block in the remaining reentrant circuit ${ }^{22-24}$ and disappearance of foci ${ }^{7}$ lead to AF termination.

Another aspect in our knowledge about AF cardioversion is the behavior of basic electrophysiological parameters, such as AF cycle length (AFCL) and conduction velocity. Prolongation of AFCL ${ }^{14-16,18-20,25-27}$ and a decrease in conduction velocity ${ }^{20,25}$ are known as common effects associated with class I and III AADs. On top of a drug effect, a sudden increase of AFCL was observed just before cardioversion of AF by cibenzoline. ${ }^{25}$ Comparable findings were reported in studies that investigated inhibition of the acetylcholine-activated potassium current $\left(\mathrm{I}_{\mathrm{KACh}}\right.$, chapter 2$)$ and the small conductance calcium-activated potassium current $\left(\mathrm{I}_{\mathrm{SK}}\right.$, chapter 3).

These consistent findings led us to hypothesize that there might be some shared mechanisms that are responsible for the organization of fibrillatory conduction and the changes in electrophysiological parameters occurring just before AF termination. To test this hypothesis, we performed an investigation of AF terminations under a variety of conditions; termination of acute AF, spontaneous termination of AF that has been maintained for 3-4 weeks and druginduced termination of persistent AF. Using a goat model of AF, we performed synchronous bi-atrial high-density electrical mapping to capture conduction patterns and changes in electrophysiological properties in the final stage before AF termination. 


\section{METHODS}

This study is a retrospective evaluation of 6 independent studies that were executed over a period of 5 years. All animal procedures were approved by the local ethical board for animal experimentation and were performed in compliance with Dutch and European directives on the use of animals for scientific purposes. Thirty-three Dutch milk goats were included in the study. General instrumentation, measurements and anesthesia were identical between the groups. An overview of the groups is summarized in Table 1. The animals from the Acute AF group ( $\mathrm{n}=4)$ were in sinus rhythm and AF was acutely induced during the experiment. In the remaining animals $(\mathrm{n}=29)$ AF was maintained by an implanted pacemaker. In these animals the experimental protocols were designed to record AF at baseline before the drug infusion and during the infusion until AF terminated. Further details regarding individual experimental protocols are provided in chapter 2 (XAF-1407), chapter 3 (AP14145) and in the studies on vernakalant ${ }^{20,21}$ and PA-6. ${ }^{19}$ Spontaneous cardioversions before the administration of antiarrhythmic compound were observed in one animal from the PA-6 study and in one animal from the XAF-1407 study. These cardioversions were included into a separate group called Spontaneous cardioversion.

Table 1. Overview of the investigated groups.

\begin{tabular}{|c|c|c|c|c|}
\hline Group / Drug & AAD class & Inhibited current & $\begin{array}{l}\text { Number } \\
\text { of goats }\end{array}$ & $\begin{array}{c}\text { Number of AF } \\
\text { terminations }\end{array}$ \\
\hline Acute AF & -- & -- & 4 & 8 \\
\hline Spontaneous cardioversion & -- & -- & 2 & 6 \\
\hline AP14145 & III & $\mathrm{I}_{\mathrm{SK}}$ & 5 & 13 \\
\hline PA-6 & III & $\mathrm{I}_{\mathrm{K} 1}$ & 7 & 17 \\
\hline Vernakalant & I & $\mathrm{I}_{\mathrm{Na}}, \mathrm{I}_{\mathrm{K}}$ & 8 & 16 \\
\hline XAF-1407 & III & $\mathrm{I}_{\mathrm{KACh}}$ & 9 & 25 \\
\hline
\end{tabular}

\section{Animal model}

A left-sided thoracotomy was performed under general anesthesia (sufentanyl $6 \mu \mathrm{g} / \mathrm{kg} / \mathrm{h}$ IV and propofol $5-10 \mathrm{mg} / \mathrm{kg} / \mathrm{h}$ IV) to implant a custom-build patch of electrodes on the pericardium overlying the left atrium (LA). One electrode pair was connected to a subcutaneous neurostimulator (Medtronic Itrel ${ }^{\oplus}$, Medtronic, Minneapolis, Minnesota, USA). The other wires were tunneled to the neck and exteriorized for monitoring of the atrial rhythm. The animals received post-operative treatment with antibiotics (ampicillin, $10 \mathrm{mg} / \mathrm{kg} \mathrm{IM}$, three times a day, first two days after the surgery) and analgesia with buprenofine (10 $\mu \mathrm{g} / \mathrm{kg}$ IM, 
twice a day, first day after the surgery) and carprofen $(2-4 \mathrm{mg} / \mathrm{kg} \mathrm{SC}$, first three days after surgery). After full recovery, AF was induced and maintained in all groups, except the Acute $A F$ group, for 3-4 weeks by burst pacing $(50 \mathrm{~Hz}, 10 \mathrm{~V}, 1 \mathrm{~s}$ on, $1 \mathrm{~s}$ off). After this period, an open chest study under general anesthesia (sufentanyl $6 \mu \mathrm{g} / \mathrm{kg} / \mathrm{h}$, propofol $10 \mathrm{mg} / \mathrm{kg} / \mathrm{h}$ and rocuronium $0.3 \mathrm{mg} / \mathrm{kg} / \mathrm{h}$ ) was performed. A left-sided thoracotomy allowed the placement of two mapping arrays (249 electrodes each, interelectrode distance $2.4 \mathrm{~mm}$ ), one on the right atrial (RA) and one on the LA free wall. Mapping arrays were kept in fixed position during the whole experiment. Unipolar electrograms were recorded at a sampling rate of $1 \mathrm{kHz}, 16$-bit resolution, filter bandwidth $0.56-408 \mathrm{~Hz}$.

\section{Sacrifice protocol}

After surgical instrumentation, a stabilization period of $\geq 30$ minutes was allowed in all animals. The animals from the Acute AF group were in SR at this point. In these animals AF was induced by a one-second burst of $50 \mathrm{~Hz}$ and automatically reinduced after the detection of SR that lasted longer than 1 second. An AF paroxysm was recorded if it lasted longer than 10 seconds. The animals in the other groups were in AF at this point of the experiment and AF stability was monitored continuously. A baseline recording of unipolar atrial electrograms during AF was acquired for 60 seconds at the end of the stabilization phase. Next, AF was terminated by internal defibrillation (Physio-Control Lifepak 9B, Medtronic, Minneapolis, Minnesota, USA) in order to perform S1-S1 and S1-S2 pacing protocols (except of the PA-6 group). These data were used for other investigations but are not analyzed further in this study. The drug administration protocol was initiated after AF reinduction and AF stabilization for at least 20 minutes. Unipolar atrial electrograms were acquired continuously during baseline and drug administration. In case AF terminated either spontaneously at baseline or during drug infusion, AF was reinitiated by the same computer-controlled algorithm as described above. This approach prevented reverse remodeling and allowed the recording of multiple AF terminations within one animal.

\section{Data analysis}

For baseline AF analysis, six consecutive 10-second periods were selected. The terminations of the first $3 \mathrm{AF}$ episodes that lasted longer than 10 seconds were considered for analysis, if available. From these terminations a time interval of 10 seconds before the last AF activation was identified and used for analysis. Custom-made software written in the MATLAB programming environment (The MathWorks, Inc., Natick, Massachusetts, USA) was used for analysis of local activation times in the unipolar atrial electrograms, applying a probabilistic annotation algorithm. ${ }^{28}$ When required, high-frequency noise and ventricular far-field effects were removed before the activation time assignment. To avoid bias for the applied drug or the order of cardioversions, the recordings were blinded to the operator. The same operator 
classified the final conduction pattern before termination. These patterns were scored for: (1) eight propagation directions (north, northeast, etc.), (2) number of wavefronts and (3) type of wave (peripheral wave, radial spread of activation or reentry).

\section{Activation time histograms}

Sequences of local activation times per electrogram were summed up for all channels per electrode array and plotted in an activation time histogram, as illustrated in Figure 1. To reduce noise, the activation histograms were binned by two samples (i.e., $2 \mathrm{~ms}$ ). The parts of the activation histograms exhibiting beat-like activity, showing clear peaks surrounded by periods without electrical activations, were considered as periodic activity. Periodic activity was formalized as an activation of the entire mapping area in one sequence of activations lasting shorter than $120 \mathrm{~ms}$ (equivalent to conduction velocity faster than $32 \mathrm{~cm} / \mathrm{s}$ for the entire map). In the activation histogram, a period of activations lasting over $120 \mathrm{~ms}$ was considered to be continuous activity. Identification of continuous and periodic activity allowed us to (1) characterize the dynamic changes in temporal organization of AF and (2) to study the beatto-beat electrophysiological properties during periodic activity, including AFCL, conduction velocity, and path length (defined as AFCL $\times$ conduction velocity).

\section{Statistics}

Kruskal-Wallis test with Dunn's multiple comparison test was used to assess the differences in electrophysiological parameters between the groups (Figure 2). The changes in AFCL, conduction velocity and path length before AF termination (Figure 6C) were evaluated by Friedman test with Dunn's multiple comparisons test. A linear mixed-effects model was used for the comparison of changes in AFCL during the last 10 seconds before AF termination (Figure 8B). Mann-Whitney test was used to compare the occurrence of continuous activity between baseline $\mathrm{AF}$ and the last seconds before $\mathrm{AF}$ termination, to compare the number of periodic AF cycles preceding AF termination between the LA and the RA (Figure 6B) and to evaluate differences in the times when the last transition from continuous to periodic activity occurred (Figure 4C). Wilcoxon test was used to compare the occurrence of continuous activity between the atria. The changes in AFCL, conduction velocity and path length in the last three periodic activities directly preceding AF termination (Figure 7B) were assessed with a chi-squared test. The value of $\mathrm{p}<0.05$ was considered to be statistically significant. All values are presented as median and interquartile range (IQR), unless stated otherwise. 


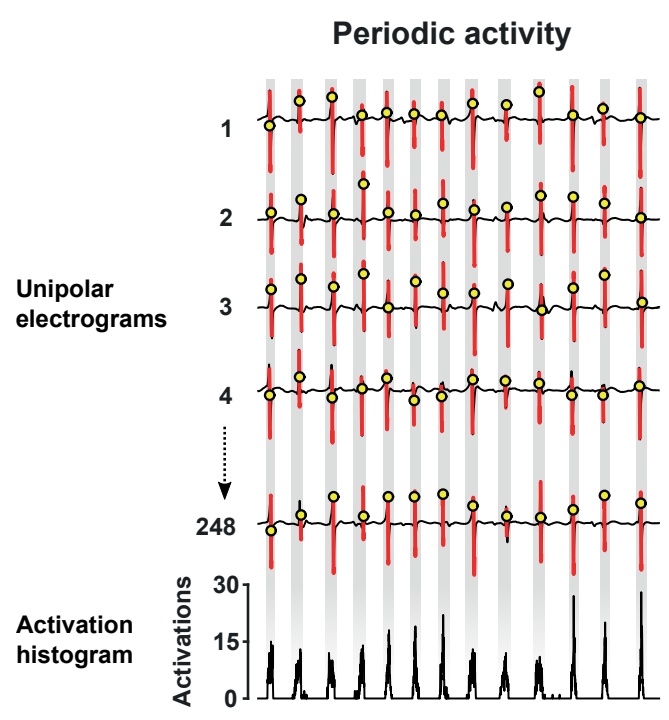

\section{Continuous activity}
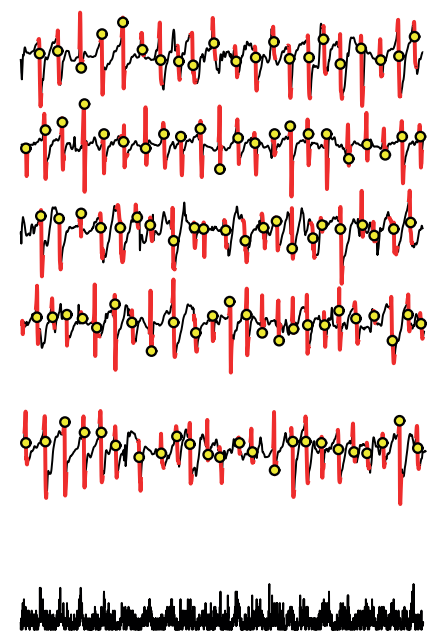

$\overline{500 \mathrm{~ms}}$

Figure 1. Construction of activation histograms. Local atrial activations were determined from unipolar electrograms. The deflections in the electrogram that corresponded with local atrial activations are depicted in red. The time points of atrial activation are indicated by yellow circles. Activation histogram represents the summation of these time points for all channels from the electrode array. Each part of the histogram with nonzero values corresponds to one or more atrial excitation waves.

\section{RESULTS}

In total, $85 \mathrm{AF}$ terminations were included in this study, as summarized in Table 1. Eight terminations were from the Acute $A F$ group and 6 terminations from the Spontaneous cardioversion group. The remaining 71 terminations were induced by AADs (13 in the group of AP14145, 17 in the group of PA-6, 16 in the group of vernakalant and 25 in the group of XAF-1407).

\section{General characterization of drug effects on AF properties}

To describe the specific drug-related effects preceding the cardioversion of AF to SR, we studied the changes in AFCL, conduction velocity and path length in both atria. We investigated the interval of 8-10 seconds before the arrhythmia termination and the drugfree baseline recording. These changes in electrophysiological parameters were considered to mainly represent the effects caused by the presence of the AAD. In addition, we studied the electrophysiological parameters from the last activation cycle before AF termination. 
Figure 2 summarizes the changes in AFCL, conduction velocity and path length for all studied groups except of the Acute AF group. Prolongation of AFCL and path length was observed in the interval of 8-10 seconds before AF termination in all groups (Figure $2 \mathrm{~A}$ and $2 \mathrm{C}$ ). Vernakalant caused more pronounced lengthening of AFCL than PA-6, as shown in Figure 2A. The last activation cycle before cardioversion exhibited even further prolongation of AFCL and path length. No significant differences in these parameters were observed between the groups for the last activation cycle before AF termination. In line with the previously reported slowing of conduction by AP14145 (chapter 3) and vernakalant, ${ }^{20}$ a reduced conduction velocity was induced by these drugs (Figure 2B). However, during the last activation cycle preceding AF cardioversion the conduction velocity increased in both atria in all groups, indicating that this sudden acceleration of atrial conduction was possibly associated with the process of arrhythmia termination.

Figure 2 demonstrates that the process of AF termination, represented by the values of the last activation cycle, was associated with pronounced changes in electrophysiological parameters. The prolongation of AFCL and path length as well as the acceleration of conduction velocity were observed consistently in all cardioversions in the different groups. This finding suggests that the final changes before AF termination were independent on the antiarrhythmic effect of the administered drug and reflect a shared process of cardioversion. Therefore, we grouped all the cardioversions for the further analyses together. Next, we characterized the final conduction pattern and the general properties of electrophysiological changes leading to AF termination.

\section{The conduction patterns preceding AF termination}

Conduction patterns immediately preceding AF termination were analyzed for the number of waves, the type of wave(s) and the number of repetitions. A non-repetitive final conduction pattern occurred in 44/85 (52\%) cases in the LA and 45/85 (53\%) cases in the RA. In the other cases the conduction patterns repeated from twice to many more $(>10)$ times. The conduction patterns generally showed 1 or 2 wavefronts prior to the termination of the arrhythmia. The conduction patterns were classified in 3 categories (Figure 3). (1) The propagation pattern with at least one wave originating from the Bachmann's bundle that activated the atrial free wall(s) (anterior to posterior conduction) was observed in 51 cases (68\%). (2) A pattern where one atrium exhibited posterior-anterior conduction and the wavefront in the contralateral atrium conducted in the opposite anterior-posterior direction was found in 17 cases (23\%). (3) The least common category (7 cases, $9 \%$ ) represents a combination of patterns, i.e., radial spread of activation in both atria with a source located in the septal area $(n=4)$, a reentrant circuit $(n=2)$, and multiple, almost synchronous focal sites $(n=1)$. In summary, 91\% (categories 1\&2) of termination patterns displayed propagation from Bachmann's bundle to the atrial free walls. 
Interestingly, 83/85 (98\%) of final conduction patterns entered and exited the field of view, suggesting that the source of final atrial activation was localized outside of the mapped region (e.g., septum, crista terminalis or Bachmann's bundle).

\section{Periodic and continuous activity during baseline AF and before AF termination}

To characterize the dynamics of temporal organization of AF, we constructed activation time histograms for baseline AF and for the last 10 seconds preceding AF termination. Figure 4A shows representative examples of activation time histograms with assigned intervals of periodic and continuous activity. During periodic activity the mapped area was generally activated by one or a few fibrillatory wavefronts at once (Figure 4B), followed by a pause when no activation was present. In contrast, multiple waves were always present in the mapped region during the intervals of continuous activity, reflecting unceasing propagation within the atrial free wall. In the activation time histograms, this behavior manifested as multiple peaks with low amplitude that followed each other.

The occurrence of continuous activity at baseline $\mathrm{AF}$ and during $\mathrm{AF}$ termination was determined. Figure $4 \mathrm{C}$ illustrates the percentage of recordings in which continuous activity was present at given time point. Continuous activity was more common at baseline AF than during the last 10 seconds before AF termination, both in the LA (28\% [IQR 12-46\%] vs. $5 \%$ [IQR 0-13\%], p<0.0001) and in the RA (65\% [IQR 42-80\%] vs. 14\% [IQR 3-26\%], $\mathrm{p}<0.0001)$. At baseline AF, continuous activity was also more prevalent in the RA than in the LA $(\mathrm{p}<0.0001)$. The overall occurrence of continuous activity remained stable during baseline AF but progressively decreased during the last 3-4 seconds before AF termination (Figure 4C). This change was also accompanied with a decrease of the interatrial gradient in the occurrence of continuous activity. 


\section{AF cycle length}

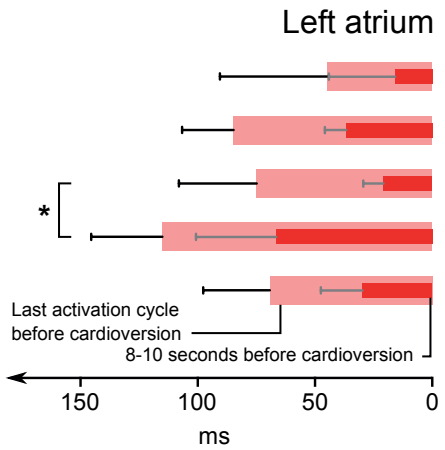

\section{Right atrium}

Spont. cardiov.

AP14145

PA-6

Vernakalant

XAF-1407
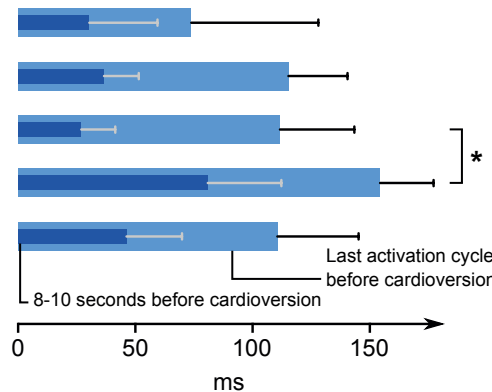

B

Conduction velocity
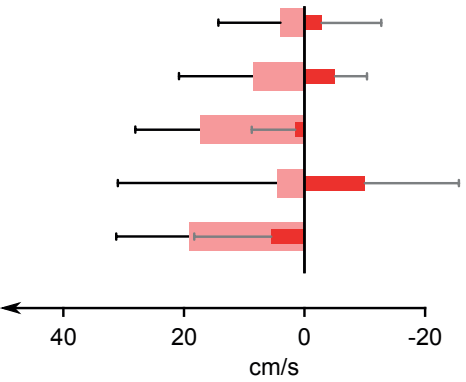

C

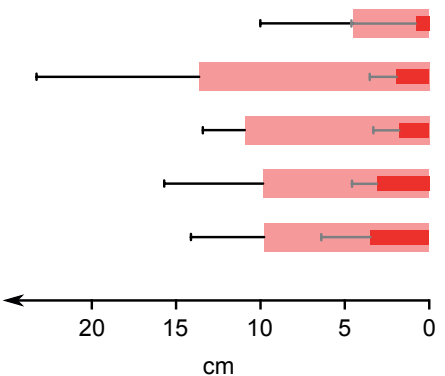

Spont. cardiov.

AP14145

PA-6

Vernakalant

XAF-1407
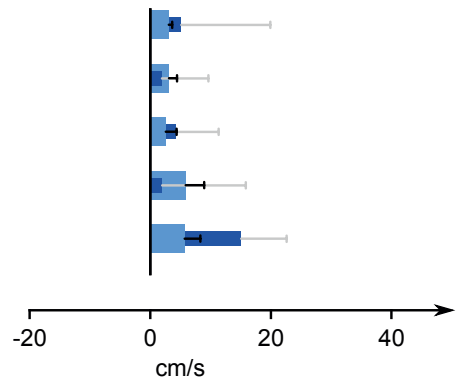

\section{Path length}

Spont. cardiov.

AP14145
PA-6

Vernakalant

XAF-1407
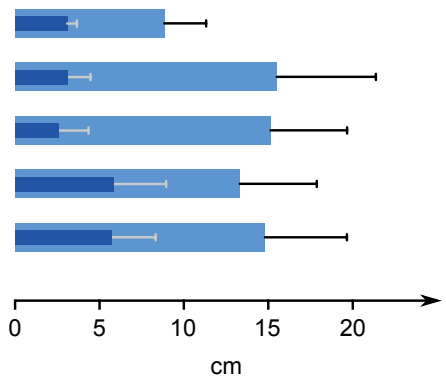

Figure 2. Comparison of electrophysiological properties between the studied groups. (A) Prolongation of AFCL was observed in all groups during the period of 8-10 seconds before AF termination when compared to baseline. Significantly longer AFCLs were found in the vernakalant group when compared to PA-6 in both atria. Within the last seconds before AF termination AFCL further prolonged, so the final AF cycle exhibited even longer AFCL. No significant differences between the groups were found for either atrium. (B) An increase in conduction velocity right before AF termination was observed in all groups. A tendency towards deceleration of conduction velocity in the period of 8-10 seconds before AF termination was observed in the group of Spontaneous cardioversions, AP14145 and vernakalant. (C) Prolongation of path length in the period of 8-10 seconds before AF termination was observed in all groups. The path length prolonged even further in the last AF cycle before AF cardioversion. 


\section{Bachmann's bundle-associated conduction \\ $(n=51,68 \%)$}

\section{Anterior-posterior conduction \\ $(n=17,23 \%)$}

\section{Unique mechanisms}

$(n=7,9 \%)$

Figure 3. Conduction patterns immediately preceding termination of atrial fibrillation (AF). Top: the most common conduction patterns were associated with a source coming from, or feeding Bachmann's bundle. Middle: the second most common pattern showed a posterior-anterior conduction in one atrium and anterior-posterior conduction in the contralateral atrium. Bottom: unique mechanisms (from left to right): radial spread of activation in both atria with a source oriented in the septal area, reentrant circuit and multiple, almost synchronous focal sites. 
A
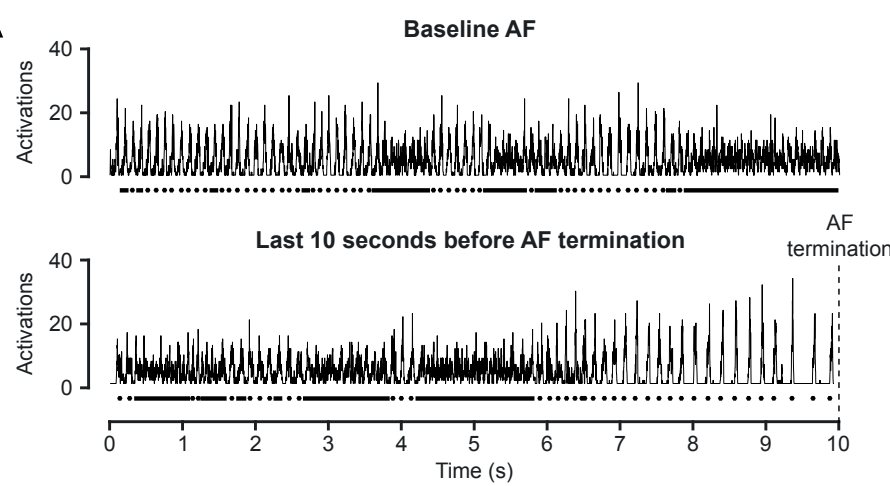

B

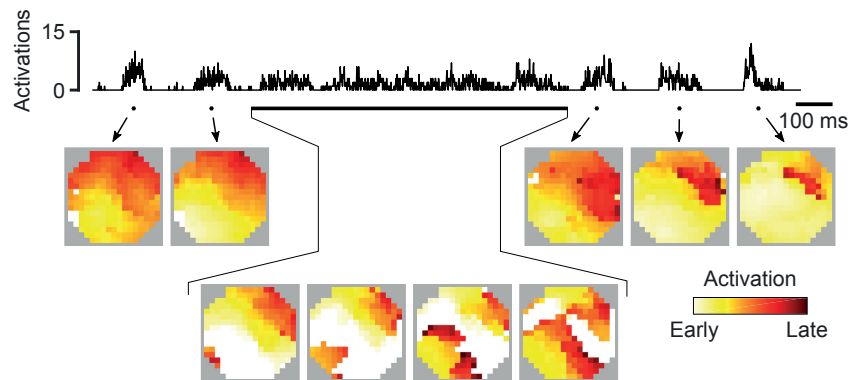

C
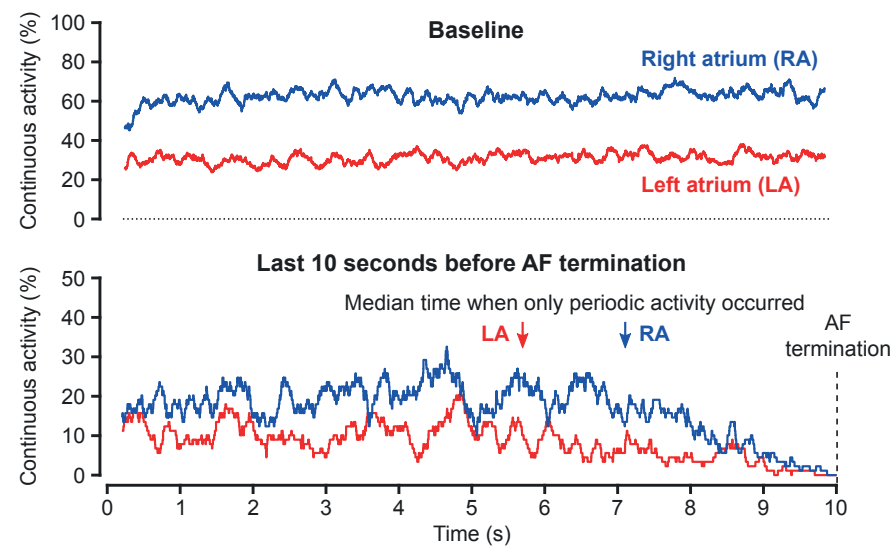

Figure 4. The occurrence of continuous and periodic activity at baseline AF and before AF termination. (A) Representative examples of activation histograms acquired at baseline AF (top) and during the last 10 seconds before AF termination (bottom). The lines under the activation histogram indicate intervals of continuous activity, the dots indicate periodic activity. (B) An example of activation histogram with assigned continuous (line) and periodic activity (dots). The activation maps illustrate underlying activation patterns. During periodic activity, the mapped region is activated by one or more excitation waves at once, followed by an interval of electrical silence. In contrast, during the episodes of continuous activity, there is always at least one excitation wave present in the mapped region, re-exciting the tissue in the mapped region continuously. (C) The occurrence of continuous activity at baseline AF and during the last 10 seconds before AF termination. The median times when continuous activity stopped and only periodic activity was present are indicated by arrows in the lower panel. 
Next, we addressed the stability of continuous and periodic activity during the last 10 seconds before AF termination (Figure 5A). Three distribution patterns were identified: (A) "high regularity" AF, characterized by continuous presence of periodic activity, (B) abundant continuous activity followed by a transition towards periodic activity during the last few seconds before AF termination and (C) intermittent periods of continuous and periodic activity. Figure 5B lists incidences of these patterns for each atrium separately and their cooccurrence. The most common pattern was represented by intermittent periods of continuous and periodic activity (pattern C) in 71\% of the cases in the LA and 55\% in the RA. In 41 cases $(48 \%)$ intermittent periods of continuous and periodic activity occurred in both atria. This finding suggests that during the last seconds before cardioversion the temporal organization of the arrhythmia was unstable.

A

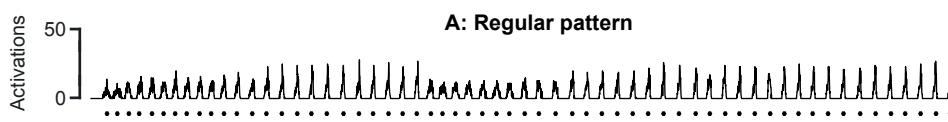
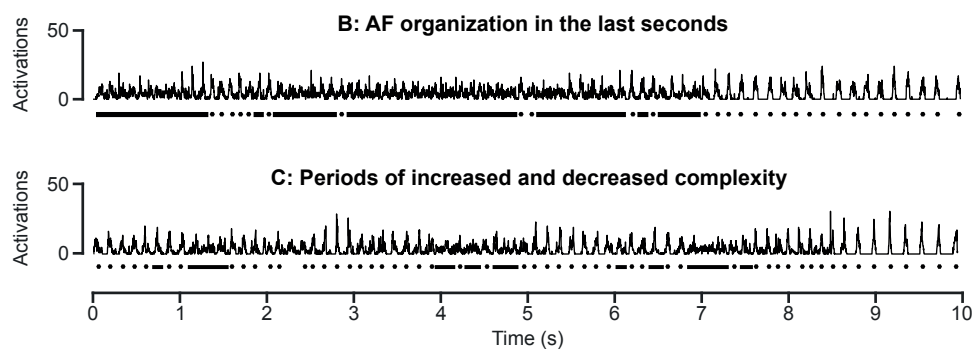

B

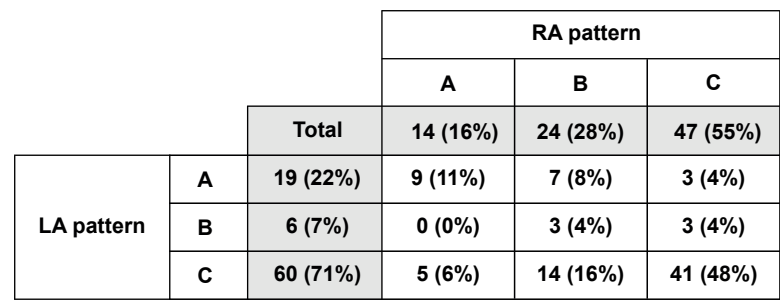

Figure 5. Patterns of periodic and continuous activity before termination of atrial fibrillation (AF). (A) Examples of patterns during the last 10 seconds before AF termination. The dots and lines represent periodic and continuous activity, respectively. Three different patterns with respect to the periodic and continuous activity were observed in the atria. Top panel: regular pattern exhibiting only periodic activity. Middle panel: abundant continuous activity that turns into periodic activity in the last few seconds before AF termination. Bottom panel: presence of continuous and periodic activity with a tendency towards oscillations between the phases with increased and decreased AF complexity, exhibited as phases with higher and lower occurrence of continuous activity, respectively. (B) The presence of the patterns in LA and RA, demonstrating that the pattern with oscillations between the increased and decreased AF complexity was the most common. 
A phase of periodic activity just prior to cardioversion of AF occurred in all studied cardioversions. Hence, continuous activity entirely disappeared just before AF termination. In many cases a considerable number of cycles with periodic activity occurred before AF termination, as illustrated in Figure 6A. The distributions of the number of periodic cycles were comparable between the LA and RA (Figure 6B)(21 [IQR 10-28] vs. 15 [IQR 10-25], LA vs. $R A$, respectively, $p=0.132$ ), indicating that there was no atrium generally leading the transition from the continuous to periodic activity.

As mentioned above, AFCL and path length substantially prolonged and conduction velocity increased during the last AF cycle before termination of the arrhythmia (Figure 2). As a next step, we investigated whether the large change in electrophysiological parameters is attributable to the occurrence of the final periodic episode. Average AFCL, conduction velocity and path length were determined for the time period preceding the final periodic episode and for the first and the last cycle from the final periodic episode (Figure 6A). Figure 6C shows that the robust prolongation of AFCL and path length as well as the acceleration of conduction velocity were primarily present during the end of the final episode of periodic activity, but not at the beginning.

\section{Oscillations in the ultimate 3 AF cycles preceding AF termination}

The consistent presence of an episode of periodic activity before the cardioversion went along with the conduction patterns exhibiting large waves. Since these patterns exhibited repetitive behavior and up to $91 \%$ of them were associated with conduction through Bachmann's bundle, we speculated that a macro-reentry involving Bachmann's bundle could have occurred during the last AF cycles before AF termination. Studies by Frame et al. have described that the termination of a macro-reentrant circuit is preceded by beat-to-beat oscillations in action potential duration and conduction velocity. ${ }^{29-31}$ Oscillations of electrophysiological parameters in the last 3 periodic cycles before the cardioversion of AF were investigated for AFCL, conduction velocity and path length. Four possible patterns of changes in electrophysiological parameters were distinguished (Figure 7A). No statistically significant preference towards any of the patterns was found for AFCL in either atrium, although there was a trend towards the "longer-longer" pattern (Figure 7B). For conduction velocity, there was a general tendency towards the oscillations in "longer-shorter" pattern (LA: 40.0\%, p=0.011; RA: 38.8\%, $\mathrm{p}=0.003$ ). For path length, a tendency towards all the patterns except of the "shorter-shorter" pattern was found (LA: $p=0.05$; RA: $p=0.01$ ). Termination of AF in goats was therefore commonly preceded by a "longer-shorter" oscillations in conduction velocity, but no consistent pattern of oscillations in AFCL and path length occurred. 
A

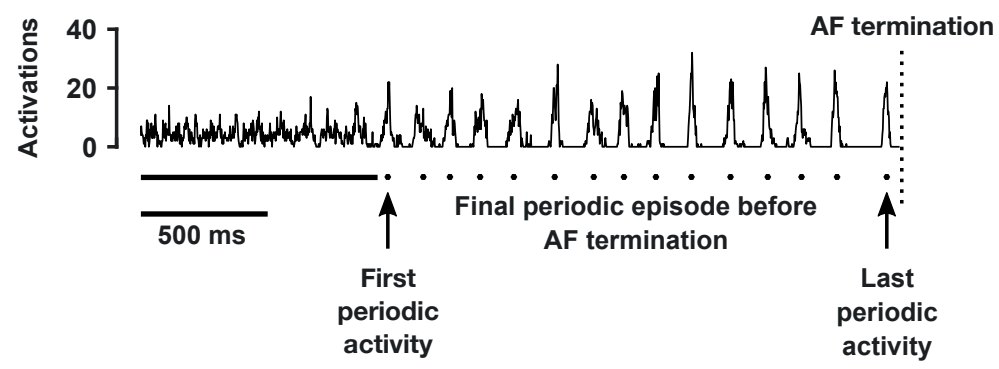

B

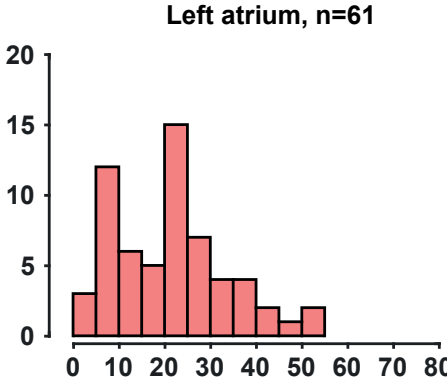

Number of periodic activities before AF termination
Right atrium, $n=68$

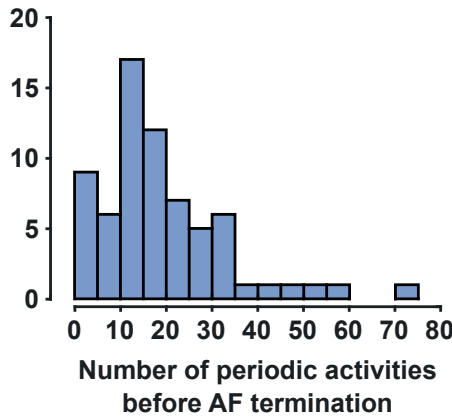

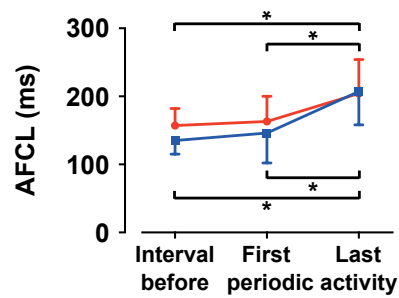
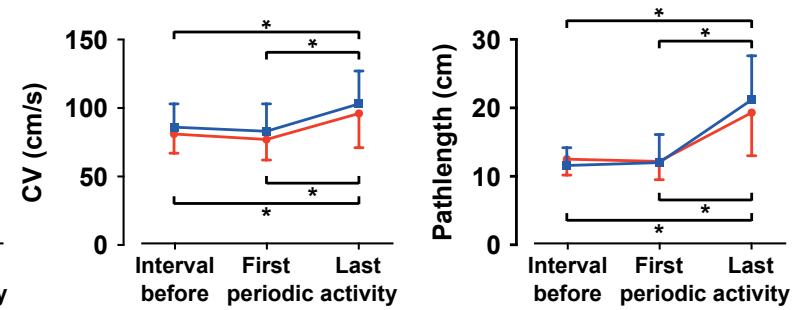

Figure 6. Changes in electrophysiological parameters during the last periodic activities preceding AF termination. (A) An example of activation histogram with an episode of continuous activity (marked with a line) followed by an episode of periodic activity (individual periodic activities are indicated by dots) immediately preceding AF termination. (B) Distributions of the number of periodic activities from the last interval of periodic activity preceding AF termination. Only the cardioversions in which a transition from continuous to periodic activity occurred were considered. There was no significant difference in the number of periodic activities between the atria right before AF termination ( $\mathrm{p}=0.132$ ). (C) Significant prolongation of AF cycle length (AFCL) accompanied with a significant increase of conduction velocity $(\mathrm{CV})$ and path length was observed at the last periodic $\mathrm{AF}$ cycle that preceded $\mathrm{AF}$ termination. 
A

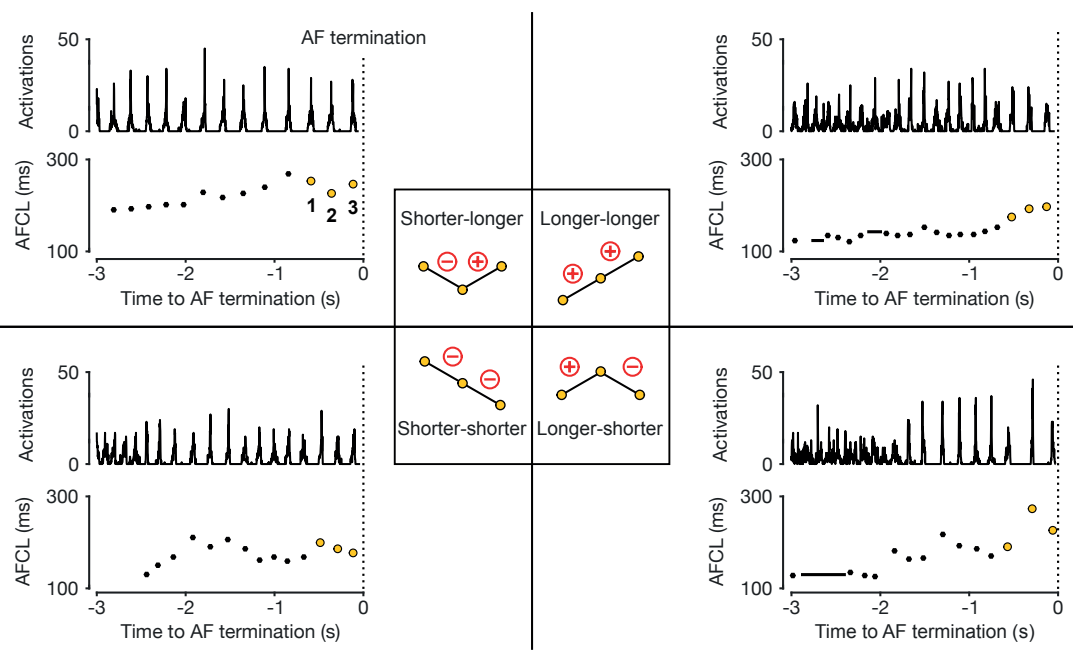

B

Left atrium
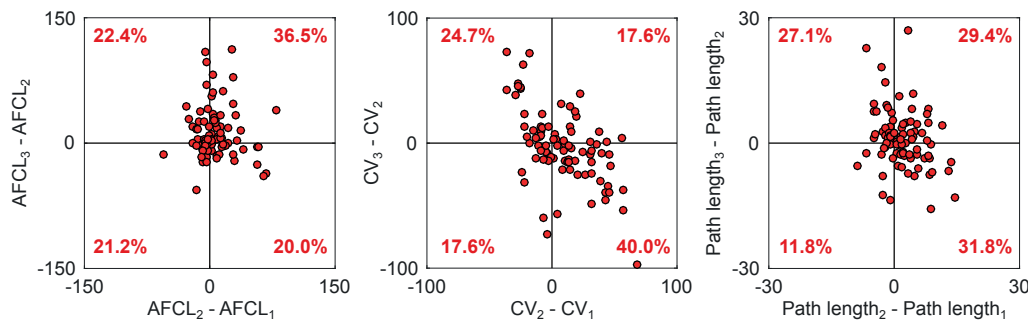

Right atrium
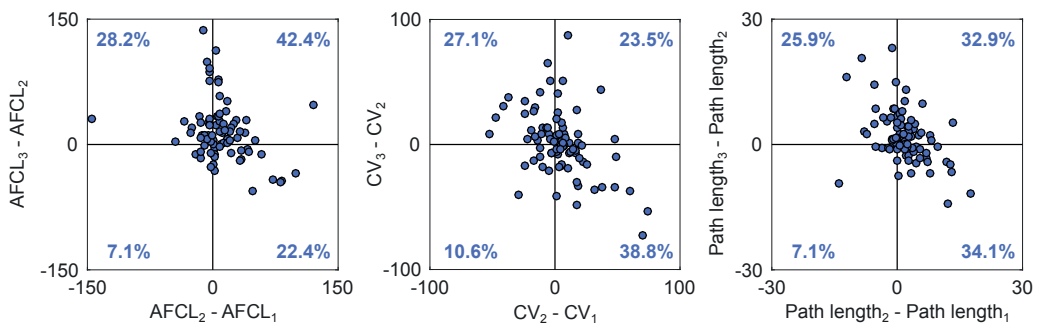

Figure 7. Patterns of changes in electrophysiological parameters in the last 3 periodic cycles before AF termination. (A) Four possible patterns that an electrophysiological parameter can exhibit during the last three periodic cycles immediately preceding AF termination (orange circles), illustrated on representative examples of $\mathrm{AF}$ cycle length (AFCL). The lines and dots represent continuous and periodic activity, respectively. (B) The occurrence of individual patterns for AFCL, conduction velocity (CV) and path length in the left atrium (LA) and in the right atrium (RA). The quadrants correspond to the panel A, each point represents a single AF termination. The indices in the axis description correspond to the order of the last periodic activities, as stated in the top left example of panel A. The numbers in each quadrant represent the occurrence of the pattern as a percentage. No statistically significant trend towards a particular pattern was observed for AFCL in either atrium. General tendency towards the "longer-shorter" pattern was observed for conduction velocity in both atria (LA: $\mathrm{p}=0.011$; RA: $\mathrm{p}=0.002$ ). For path length, there was a general tendency towards all patterns except of the "shorter-shorter" pattern (LA: $p=0.05$; RA: $p=0.01$ ). 


\section{Inter-atrial gradients and low-frequency oscillations in AFCL}

The absence of a consistent oscillation pattern of AFCL preceding cardioversion of AF led us to investigate the temporal changes in AFCL further. As the changes in AFCL on the level of individual atria were not indicative for any particular mechanism underlying AF termination, we focused on interaction of changes in AFCL between the atria. We studied the presence of inter-atrial gradients and temporal instabilities of $\mathrm{AFCL}^{26}$ during the last 10 seconds before $\mathrm{AF}$ termination (Figure 8A). Performing a qualitative assessment of changes in AFCL, we found out that 36 (42\%) cardioversions showed an inter-atrial gradient of AFCL, predominantly with the RA exhibiting a shorter AFCL than the LA. Temporary prolongation or instability of AFCL in one atrium that was not found in the contralateral atrium was observed in 25 (29\%) cardioversions. No apparent temporal instabilities in AFCL with no inter-atrial gradient were observed in 24 (28\%) cardioversions. A common phenomenon that preceded the vast majority of cardioversions was an increase in AFCL in both atria during the last cycles before $\mathrm{AF}$ termination. This observation is consistent with the findings presented in Figure $2 \mathrm{~A}$ and Figure 6C, showing prolongation of AFCL right before termination of the arrhythmia. When present, the inter-atrial gradient of AFCL diminished during this period, indicating that synchronization of AFCL between the atria preceded AF termination. Further illustrating this phenomenon, Figure $8 \mathrm{~B}$ shows the average AFCL per atrium, quantified in non-overlapping windows of $250 \mathrm{~ms}$ for all 85 cardioversions. An inter-atrial gradient in AFCL was observed, with AFCL in LA being significantly longer than in RA. During the last three seconds before cardioversion the AFCL started to prolong in both atria and the inter-atrial gradient tended to disappear. AF terminations in the goat model of AF were thus preceded not only by prolongation of AFCL and acceleration of conduction velocity, but also by disappearance of inter-atrial gradient and synchronization of AFCL between the atria. 
A
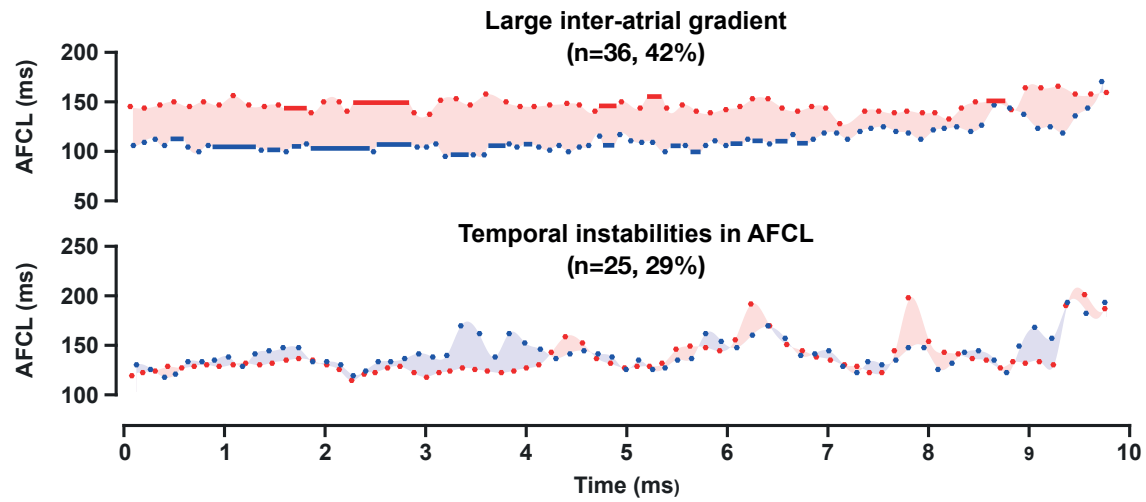

B

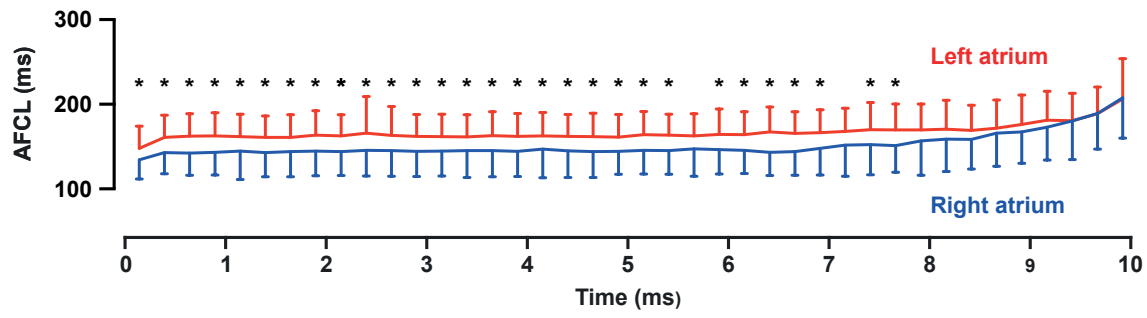

Figure 8. Changes in atrial fibrillation (AF) cycle length (AFCL) during the last 10 seconds before AF termination. (A) Top panel: an example of large inter-atrial gradient in AFCL that gradually diminished prior to AF termination. Bottom panel: temporal instabilities in AFCL in one atrium that were not followed by the contralateral atrium. AF termination was preceded by a mutual increase of AFCL in both atria. The dots and lines represent periodic and continuous activity, respectively. Red area indicates that AFCL in LA is longer than in RA, blue area indicates longer AFCL in RA. (B) Average AFCLs for each atrium during the last 10 seconds before AF termination show that the interatrial gradient in AFCL had a tendency to disappeared before cardioversion. The values are calculated from intervals of 250 milliseconds. Statistically significant differences between the atria at individual time points are indicated $\left({ }^{*} \mathrm{p}<0.05\right)$.

\section{DISCUSSION}

\section{Major findings}

Performing a retrospective analysis of several cardioversion studies, this work shows that there are common mechanisms contributing to $\mathrm{AF}$ termination in goats. These mechanisms can be generally described by increased spatiotemporal organization of the fibrillatory conduction during the last seconds before cardioversion. This process was accompanied with a profound increase of AFCL and acceleration of atrial conduction, resulting in a prolongation of path 
length. Conduction patterns became simple in both atria and the direction of propagation in atrial free walls often originated from the Bachmann's bundle. These findings suggest that AF termination was not a random process but follows common spatiotemporal patterns.

\section{The effects of AADs on fibrillatory conduction before AF termination}

Prolongation of AFCL is a common effect of $\mathrm{AADs}^{32}$ and has been demonstrated for several compounds in the goat model of pacing-induced AF. ${ }^{14-16,18-20,25-27}$ The changes in conduction velocity, however, depend on the class of AAD. Antiarrhythmic compounds exhibiting class I properties generally cause deceleration of conduction velocity while class III drugs leave the atrial conduction unaffected. ${ }^{32}$ Studying the changes in electrophysiological parameters before cardioversion, we observed that the last 10 seconds before the arrhythmia extinction were characterized by prolongation of AFCL in all studied groups. Interestingly, this included prolongation of AFCL also in the group of Spontaneous cardioversions, indicating that AF termination was generally preceded by prolongation of AFCL, irrespective of the presence of AAD.

An increased spatiotemporal organization of AF was observed in all studied cardioversions during the last few seconds before the arrhythmia termination. This period was associated with presence of relatively simple conduction patterns in the atrial free walls, sudden prolongation of AFCL and acceleration of atrial conduction in both atria. Interestingly, conduction velocity increased before pharmacological cardioversions, even in case of vernakalant, a welldocumented class I AAD. ${ }^{20}$ The mechanism underlying this effect remains unknown. The dependency of conduction velocity on AFCL might be a possible explanation. As AFCL prolonged, acceleration of atrial conduction is conceivable, resulting in prolongation of path length and disappearance of the excitable gap. These phenomena led to synchronization of atrial activity, manifested in the activation histograms as presence of periodic activity. Our observations are in agreement with a previous study in the same animal model, describing sudden prolongation of AFCL shortly before cardioversion with a class IC drug cibenzoline, associated with decreased electrogram fractionation that indicates reduced AF complexity. ${ }^{25}$ As the sudden prolongation of AFCL and acceleration of atrial conduction preceding AF termination occurred regardless of the presence or the class of $\mathrm{AAD}$, our findings suggest that the process of AF termination in goats is intrinsically associated with abrupt changes in electrophysiological parameters.

\section{Conduction patterns preceding the cardioversion of AF to SR}

To our knowledge, there have been only few studies that used atrial mapping to describe the process of AF termination. The most extensive investigations were presented in studies by Wang et al., describing conduction patterns that preceded cardioversions with different 
AADs. ${ }^{22-24}$ The authors investigated 41 cardioversions in a canine model of vagally-induced AF, performing low density (112 bipolar electrodes) but high-coverage mapping of atria. They reported reduction in the number of simultaneously present reentrant circuits prior to cardioversion. Arrhythmia termination was caused by a conduction block in the critical part of the reentrant circuit $(27 / 41,66 \%)$, or by excitation of the tissue within the reentrant pathway from a distant wave $(14 / 41,34 \%)$. However, no preferential site of termination was observed. The authors reported comparable mechanisms of cardioversion also in six dogs in which AF was sustained even in the absence of cholinergic activation. ${ }^{33}$ Interestingly, the recent study by Lee et al. in the same canine model of vagally-induced AF came to a different conclusion. ${ }^{7}$ Applying epicardial mapping with higher density (510 unipolar electrograms), the authors described that the extinction of arrhythmia occurred as a result of the disappearance of focal activations.

The goat model of pacing-induced AF that has been used in this study is independent of cholinergic activation. In contrast to the above-mentioned studies, we mapped only the atrial free walls, but with substantially higher electrode density. In the vast majority of cardioversions, we observed fibrillatory waves entering and exiting the mapped region, indicating that the mechanism underlying AF termination, either the conduction block or focal source maintaining AF, was present outside of the mapped region. As both of these mechanisms can be possibly preceded by prolongation of AFCL and increase in atrial conduction, our study does not allow to draw a conclusion which of these mechanisms was more dominant in our model. Nevertheless, our findings indicate that the site where the critical event underlying termination of the arrhythmia occurred was not entirely random but might have been linked to anatomical structures or areas. Considering the fact that in $91 \%$ of cardioversions a propagation in anteriorto-posterior direction was observed, our findings are highly suggestive that Bachmann's bundle played an important role in the last activation cycles preceding AF termination.

\section{The role of Bachmann's bundle in atrial conduction and AF termination}

Bachmann's bundle is a muscular bundle comprising of parallel strands of atrial muscle fibers. It is considered as the main pathway of interatrial conduction, with its extensions bifurcating into the endocardial trabecular network of atrial free walls. ${ }^{34}$ Compared to normal atrial cardiomyocytes, the myocytes in Bachmann's bundle exhibit fast atrial conduction ${ }^{35}$ and longer atrial effective refractory periods. ${ }^{36}$ The disruption of conduction in Bachmann's bundle is known to be associated with increased vulnerability to atrial tachyarrhythmias. ${ }^{37,38}$ Although the exact mechanisms have not been described, it is conceivable that disturbed conduction in Bachmann's bundle results in dissociation of activity between atria. It is therefore likely that increased organization of conduction in Bachmann's bundle may result in fast conduction and longer path lengths in atrial free walls, which are the findings that we observed during the 
last cycles before AF termination. In addition, organized conduction in Bachmann's bundle may result in more synchronized activation of atrial free walls via the endocardial trabeculated network. Recording the atrial activations from the epicardial side, this synchronization may appear as acceleration of conduction velocity. On the other hand, the conduction through Bachmann's bundle that occurred just before AF termination might be also seen as a direct consequence of increased path length. As the path length prolongs, fibrillatory conduction can be maintained only through an anatomical pathway of sufficient size, a size that can be obtained only if the Bachmann's bundle forms part of a macro reentrant circuit. A further prolongation of the path length would lead to disappearance of the excitable gap and arrhythmia termination. Regardless of the mechanism, our observations support the previous findings that Bachmann's bundle-facilitated conduction plays an important role in atrial tachyarrhythmias.

\section{Mechanisms underlying termination of macro-reentrant circuits}

Reentry is commonly considered as one of the most important mechanisms underlying AF maintenance. ${ }^{1-4}$ The studies by Frame et al. have shown that beat-to-beat oscillations in action potential duration and conduction velocity precede termination of reentrant circuits in canine tricuspid preparations. ${ }^{29-31}$ The presence of cycle length oscillations was also observed prior to termination of atrial flutter in the model of sterile pericarditis by Ortiz et al. ${ }^{31,39}$ In our study, we studied oscillations in AFCL, conduction velocity and path length in order to investigate whether these mechanisms apply in the goat model of pacing-induced AF as well. We found that oscillations in conduction velocity preceded some of the cardioversions, indicating that termination of macroscopic reentry might have been responsible for extinction of the arrhythmia. However, as these oscillations did not occur consistently, the mechanisms underlying AF termination in the remaining cardioversions remain unclear. The absence of oscillations in electrophysiological parameters does not exclude the possibility that the remaining cardioversions were caused by termination of reentrant circuit. Other mechanisms have been described to cause interruption of reentry, including echo waves ${ }^{40}$ shortening of the functional line of block or conduction failure in the common pathway of the conduction circuit. ${ }^{41}$ The contribution of these mechanisms could not be investigated because such investigation would require electrode mapping arrays that cover the entire circuit of the reentry.

\section{LIMITATIONS}

The electrode arrays applied for electrical mapping allowed us to study the fibrillatory conduction in vivo with high spatial resolution. Being positioned on atrial free walls, they provided coverage of approximately $50 \%$ of the atrial surface. The atrial conduction occurring in the remaining parts of the atria thus remained unmapped, including the regions where the critical events underlying termination of the arrhythmia took place. Mapping with higher 
atrial coverage would be necessary to provide evidence whether the conduction via Bachmann's bundle precedes AF termination. In addition, applying analysis tools that can distinguish focal and reentrant activity could further explain the fundamental mechanisms of cardioversion.

We have shown that some shared mechanisms are associated with AF termination. However, more specific mechanisms could vary among the studied groups. To investigate these differences, detailed analysis of the effects in the individual groups would be required.

\section{CONCLUSION}

Cardioversions in the goat model of AF were always preceded by increased spatiotemporal organization of fibrillatory conduction during the last few seconds before the arrhythmia termination. This process was associated with considerable prolongation of AFCL and acceleration of atrial conduction, resulting in a profound prolongation of path length that could potentially facilitate synchronization of atrial conduction. Propagation of fibrillatory waves in anterior-to-posterior direction was consistently observed during the last AF cycles, suggesting a pivotal role of Bachmann's bundle in the last activation cycles preceding AF termination. 


\section{REFERENCES}

1. Narayan SM, Krummen DE, Shivkumar K, Clopton P, Rappel WJ, Miller JM. Treatment of atrial fibrillation by the ablation of localized sources: CONFIRM (Conventional Ablation for Atrial Fibrillation With or Without Focal Impulse and Rotor Modulation) trial. J Am Coll Cardiol 2012;60:628-636.

2. Haissaguerre M, Hocini M, Denis A, Shah AJ, Komatsu Y, Yamashita S, et al. Driver domains in persistent atrial fibrillation. Circulation 2014;130:530-538.

3. Mandapati R, Skanes A, Chen J, Berenfeld O, Jalife J. Stable Microreentrant Sourcees as a Mechanism of Atrial Fibrillation in the Isolated Sheep Heart. Circulation 2000;101:194-199.

4. Hansen BJ, Zhao J, Csepe TA, Moore BT, Li N, Jayne LA, et al. Atrial fibrillation driven by microanatomic intramural re-entry revealed by simultaneous sub-epicardial and sub-endocardial optical mapping in explanted human hearts. Eur Heart J 2015;36:2390-2401.

5. Haïssaguerre M, Jaïs P, Shah DC, Takahashi A, Hocini M, Quiniou G, et al. Spontaneous initiation of atrial fibrillation by ectopic beats originating in the pulmonary veins. $N$ Engl J Med 1998;339:659-666.

6. Lee S, Sahadevan J, Khrestian CM, Cakulev I, Markowitz A, Waldo AL. Simultaneous Biatrial High-Density (510-512 Electrodes) Epicardial Mapping of Persistent and Long-Standing Persistent Atrial Fibrillation in Patients: New Insights Into the Mechanism of Its Maintenance. Circulation 2015;132:2108-2117.

7. Lee S, Khrestian CM, Sahadevan J, Waldo AL. Reconsidering the Multiple Wavelet Hypothesis of Atrial Fibrillation. Heart Rhythm 2020.

8. Moe GK, Abildskov JA. Atrial fibrillation as a self-sustaining arrhythmia independent of focal discharge. Am Heart J 1959;58:59-70.

9. de Groot N, van der Does L, Yaksh A, Lanters E, Teuwen C, Knops P, et al. Direct Proof of EndoEpicardial Asynchrony of the Atrial Wall During Atrial Fibrillation in Humans. Circ Arrhythm Electrophysiol 2016;9:e003648.

10. Eckstein J, Maesen B, Linz D, Zeemering S, van Hunnik A, Verheule S, et al. Time course and mechanisms of endo-epicardial electrical dissociation during atrial fibrillation in the goat. Cardiovasc Res 2011;89:816-824.

11. Gharaviri A, Bidar E, Potse M, Zeemering S, Verheule S, Pezzuto S, et al. Epicardial Fibrosis Explains Increased Endo-Epicardial Dissociation and Epicardial Breakthroughs in Human Atrial Fibrillation. Front Physiol 2020;11:68.

12. Allessie M, de Groot N. CrossTalk opposing view: Rotors have not been demonstrated to be the drivers of atrial fibrillation. J Physiol 2014;592:3167-3170.

13. Narayan SM, Jalife J. CrossTalk proposal: Rotors have been demonstrated to drive human atrial fibrillation. J Physiol 2014;592:3163-3166.

14. Wijffels MCEF, Dorland R, Allessie MA. Pharmacologic Cardioversion of Chronic Atrial Fibrillation in the Goat by Class Ia, Ic and III Drugs: A Comparison Between Hydroquinidine, Cibenzoline, Flecainide and d-Sotalol. J Cardiovasc Electrophysiol 1999;10:178-193. 
15. Blaauw Y, Gogelein H, Tieleman RG, van Hunnik A, Schotten U, Allessie MA. "Early” class III drugs for the treatment of atrial fibrillation: efficacy and atrial selectivity of AVE0118 in remodeled atria of the goat. Circulation 2004;110:1717-1724.

16. Blaauw Y, Schotten U, van Hunnik A, Neuberger HR, Allessie MA. Cardioversion of persistent atrial fibrillation by a combination of atrial specific and non-specific class III drugs in the goat. Cardiovasc Res 2007;75:89-98.

17. de Haan S, Greiser M, Harks E, Blaauw Y, van Hunnik A, Verheule S, et al. AVE0118, blocker of the transient outward current $\left(\mathrm{I}_{\mathrm{to}}\right)$ and ultrarapid delayed rectifier current $\left(\mathrm{I}_{\text {Kur }}\right)$, fully restores atrial contractility after cardioversion of atrial fibrillation in the goat. Circulation 2006;114:1234-1242.

18. Eijsbouts S, Ausma J, Blaauw Y, Schotten U, Duytschaever M, Allessie MA. Serial cardioversion by class IC Drugs during 4 months of persistent atrial fibrillation in the goat. J Cardiovasc Electrophysiol 2006;17:648-654.

19. Ji Y, Varkevisser R, Opacic D, Bossu A, Kuiper M, Beekman JDM, et al. The inward rectifier current inhibitor PA-6 terminates atrial fibrillation and does not cause ventricular arrhythmias in goat and dog models. Br J Pharmacol 2017;174:2576-2590.

20. van Hunnik A, Lau DH, Zeemering S, Kuiper M, Verheule S, Schotten U. Antiarrhythmic effect of vernakalant in electrically remodeled goat atria is caused by slowing of conduction and prolongation of postrepolarization refractoriness. Heart Rhythm 2016;13:964-972.

21. van Hunnik A, Nasrallah H, Lau DH, Kuiper M, Verheule S, Schotten U. Vernakalant does not alter early repolarization or contractility in normal and electrically remodelled atria. Europace 2018;20:140-148.

22. Wang J, Bourne GW, Wang Z, Villemaire C, Talajic M, Nattel S. Comparative mechanisms of antiarrhythmic drug action in experimental atrial fibrillation. Importance of use-dependent effects on refractoriness. Circulation 1993;88:1030-1044.

23. Wang J, Feng J, Nattel S. Class III antiarrhythmic drug action in experimental atrial fibrillation. Differences in reverse use dependence and effectiveness between $\mathrm{d}$-sotalol and the new antiarrhythmic drug ambasilide. Circulation 1994;90:2032-2040.

24. Wang Z, Pagé P, Nattel S. Mechanism of flecainide's antiarrhythmic action in experimental atrial fibrillation. Circ Res 1992;71:271-287.

25. Shan Z, van der Voort PH, Blaauw Y, Duytschaever M, Allessie MA. Fractionation of electrograms and linking of activation during pharmacologic cardioversion of persistent atrial fibrillation in the goat. J Cardiovasc Electrophysiol 2004;15:572-580.

26. de Brito Santos PE, Duytschaever M, Allessie MA. Low-frequency oscillations of atrial fibrillation cycle length in goats: characterization and potentiation by class III antiarrhytmic almokalant. J Electrocardiol 2008;41:711-723.

27. Verheule S, Tuyls E, van Hunnik A, Kuiper M, Schotten U, Allessie M. Fibrillatory conduction in the atrial free walls of goats in persistent and permanent atrial fibrillation. Circ Arrhythm Electrophysiol 2010;3:590-599.

28. Zeemering S, Maesen B, Nijs J, Lau DH, Granier M, Verheule S, et al. Automated quantification of atrial fibrillation complexity by probabilistic electrogram analysis and fibrillation wave reconstruction. Conf Proc IEEE Eng Med Biol Soc 2012;2012:6357-6360. 
29. Frame LH, Rhee EK. Spontaneous termination of reentry after one cycle or short nonsustained runs. Role of oscillations and excess dispersion of refractoriness. Circ Res 1991;68:493-502.

30. Frame LH, Simson MB. Oscillations of conduction, action potential duration and refractoriness. A mechanism for spontaneous termination of reentrant tachycardias. Circulation 1988;78:12771287.

31. Ortiz J, Igarashi M, Gonzalez HX, Laurita K, Rudy Y, Waldo AL. Mechanism of spontaneous termination of stable atrial flutter in the canine sterile pericarditis model. Circulation 1993;88:18661877.

32. Workman AJ, Smith GL, Rankin AC. Mechanisms of termination and prevention of atrial fibrillation by drug therapy. Pharmacol Ther 2011;131:221-241.

33. Wang Z, Feng J, Nattel S. Idiopathic atrial fibrillation in dogs: electrophysiologic determinants and mechanisms of antiarrhythmic action of flecainide. J Am Coll Cardiol 1995;26:277-286.

34. van Campenhout MJ, Yaksh A, Kik C, de Jaegere PP, Ho SY, Allessie MA, et al. Bachmann's bundle: a key player in the development of atrial fibrillation? Circ Arrhythm Electrophysiol 2013;6:10411046.

35. Wagner ML, Lazzara R, Weiss RM, Hoffman BF. Specialized conducting fibers in the interatrial band. Circ Res 1966;18:502-518.

36. Duytschaever M, Danse P, Eysbouts S, Allessie M. Is there an optimal pacing site to prevent atrial fibrillation?: an experimental study in the chronically instrumented goat. J Cardiovasc Electrophysiol 2002;13:1264-1271.

37. Ariyarajah V, Fernandes J, Kranis M, Apiyasawat S, Mercado K, Spodick DH. Prospective evaluation of atrial tachyarrhythmias in patients with interatrial block. Int J Cardiol 2007;118:332-337.

38. Bayés de Luna A, Cladellas M, Oter R, Torner P, Guindo J, Martí V, et al. Interatrial conduction block and retrograde activation of the left atrium and paroxysmal supraventricular tachyarrhythmia. Eur Heart J 1988;9:1112-1118.

39. Ortiz J, Niwano S, Abe H, Rudy Y, Johnson NJ, Waldo AL. Mapping the conversion of atrial flutter to atrial fibrillation and atrial fibrillation to atrial flutter. Insights into mechanisms. Circ Res 1994;74:882-894.

40. Brugada J, Boersma L, Abdollah H, Kirchhof C, Allessie M. Echo-wave termination of ventricular tachycardia. A common mechanism of termination of reentrant arrhythmias by various pharmacological interventions. Circulation 1992;85:1879-1887.

41. Schmitt H, Wit AL, Coromilas J, Waldecker B. Mechanisms for spontaneous termination of monomorphic, sustained ventricular tachycardia: results of activation mapping of reentrant circuits in the epicardial border zone of subacute canine infarcts. J Am Coll Cardiol 1998;31:460-472. 


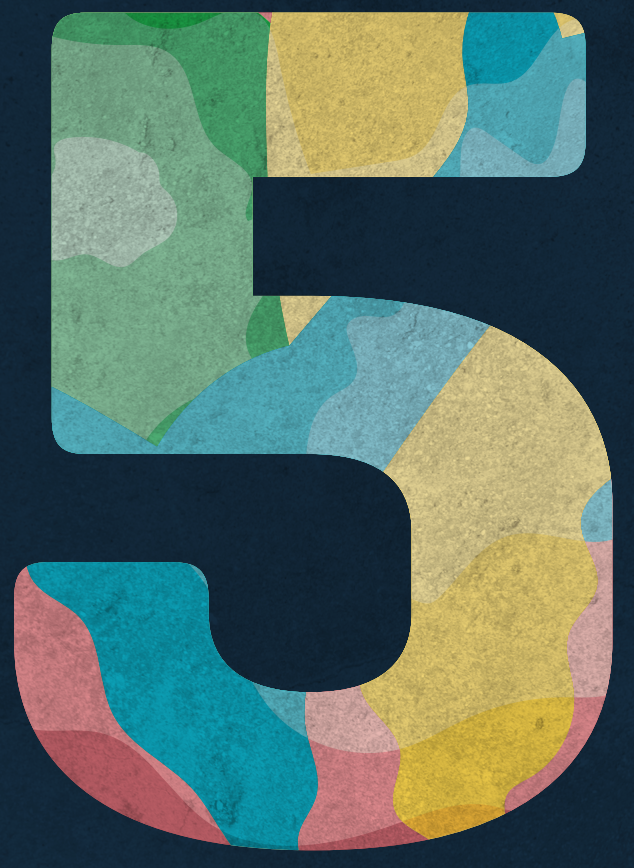




\section{A novel method for detection of atrial activations in optical mapping recordings of atrial fibrillation}

Vladimír Sobota, Marion Kuiper, Arne van Hunnik, Stef Zeemering, Ulrich Schotten and Sander Verheule

In preparation 


\section{Abstract}

Background: Optical mapping has been used to reconstruct activation patterns during cardiac arrhythmias. During complex fibrillatory conduction, slow discontinuous conduction is associated with low-amplitude optical action potentials (OAPs) with fractionated, slow upstrokes that may remain undetected by the conventional methods for activation detection. These missed detections may substantially affect the analysis of conduction patterns. We present a method for detection of atrial OAPs during atrial fibrillation (AF) using a propagation-based algorithm, with a particular focus on identification of OAPs exhibiting low magnitudes and slow upstroke.

Methods: Perfused hearts from goats that underwent either 3 weeks or 6 months of pacinginduced AF were used. Optical action potentials (100x100 pixels) were acquired in a $2 \times 2 \mathrm{~cm}$ area of the left atrial free wall during AF. Atrial activations were identified by a combination of sensitive template matching and spatiotemporal filtering. To demonstrate the ability of the method to detect small fibrillatory waves, it was compared with a standard algorithm based on thresholding of the first derivative of the fluorescence signal ( $\mathrm{dF} / \mathrm{dt}>$ threshold).

Results: Compared to the method based on $\mathrm{dF} / \mathrm{dt}$ thresholding, the presented method has a higher sensitivity to detect OAPs with slow upstrokes and low magnitude. It is capable to detect otherwise missed atrial activations and therefore improves reconstruction of excitation waves during AF, including small, slowly propagating fibrillatory waves.

Conclusion: This work presents a method for detection of atrial OAPs during AF. Applying sensitive template matching and propagation-based identification of atrial activations by spatiotemporal filtering, the method is capable to reveal small fibrillatory waves occurring during slow and discontinuous conduction. These small conducting waves remained undetected with the conventional $\mathrm{dF} / \mathrm{dt}$ threshold method. The algorithm therefore represents a novel tool for unmasking the full complexity of fibrillatory conduction. 


\section{INTRODUCTION}

Atrial fibrillation (AF) is it the most common sustained cardiac arrhythmia, affecting approximately $3 \%$ of the population aged 20 years and older. ${ }^{1}$ Being a major cause of stroke, heart failure and overall cardiovascular morbidity, ${ }^{2-4}$ it represents a large socioeconomic burden. ${ }^{4}$ The progressive nature of $\mathrm{AF}$, characterized by gradual prolongation of AF episodes, is associated with proarrhythmic remodeling of atria ${ }^{5,6}$ which causes fibrillatory conduction to become more complex and AF more stable over time..$^{7-9}$ The arrhythmogenic mechanisms underlying perpetuation of AF are still incompletely understood, creating a demand for methods that can enhance our understanding of these mechanisms. Optical mapping with voltage-sensitive dyes has provided valuable insights into the complex mechanisms of arrhythmias, including AF. ${ }^{10-12}$ It allows recording of optical action potentials (OAPs) from the surface of intact isolated hearts with high spatial and temporal resolution. Due to the physical principle of data acquisition, optical mapping has several advantages over electrode-based mapping methods, including absence of electrical far-field effects, interference from electrical stimulation ${ }^{13}$ and possibility to determine repolarization. ${ }^{14,15}$

Several software packages have been developed to analyze optical mapping data. ${ }^{12,16,17}$ However, most of them were optimized for analysis of regular rhythms, typically acquired during pacing. ${ }^{16,17}$ Optical mapping recordings of pacing commonly show a single excitation wave propagating through the mapped region and OAPs with high amplitudes and steep upstrokes, separated by intervals of full repolarization. However, during arrhythmia the excitation pattern becomes more complex, with multiple fibrillatory waves present simultaneously in the mapped region, and the conduction becoming slow and discontinuous. ${ }^{18}$ As illustrated in Figure 1, these situations may be associated with OAPs exhibiting low amplitudes and slow upstrokes, and diastolic intervals that do not reach full repolarization. Several existing algorithms derive information on local activation times on a pixel-by-pixel basis e.g., using a criterion based on amplitude and/or the first derivative of the fluorescence signal.. ${ }^{16,17}$ This allows detection of OAPs with high amplitudes and steep upstrokes, but OAPs typically associated with slow, discontinuous propagation may escape detection.

Descriptions of complex activation patterns during AF with existing optical mapping methods are rare. There is no available method that is tailored for detection of OAPs with variable morphologies that are often present during AF, leaving the complexity of patterns partially hidden. The algorithms designed for cardiac arrhythmias are still relatively scarce, ${ }^{10,12}$ focusing mainly on analysis of the arrhythmogenic mechanisms in the frequency ${ }^{16,19}$ or phase domain. ${ }^{20,21}$ The purpose of this work is to develop a method that is optimized for detection of OAPs that were acquired during complex irregular rhythms. We based our approach on template matching that has been successfully used for detection of activations in high-density 
electrical mapping of $\mathrm{AF}^{22}$ By design, template matching was performed on each pixel trace in an overly sensitive manner, creating numerous false positive activations. To eliminate false positive activations while retaining true positive activations, we made use of the principle that excitation waves propagate. Because of that, atrial activations forming a fibrillatory wave are grouped together in time and space, which distinguishes them from randomly occurring false detections due to residual noise or artefacts. Therefore, we used the information provided from neighboring pixels by applying spatiotemporal filtering of candidate OAP upstrokes. The performance of the presented method is illustrated on optical mapping data from perfused hearts of goats with persistent AF. ${ }^{9}$

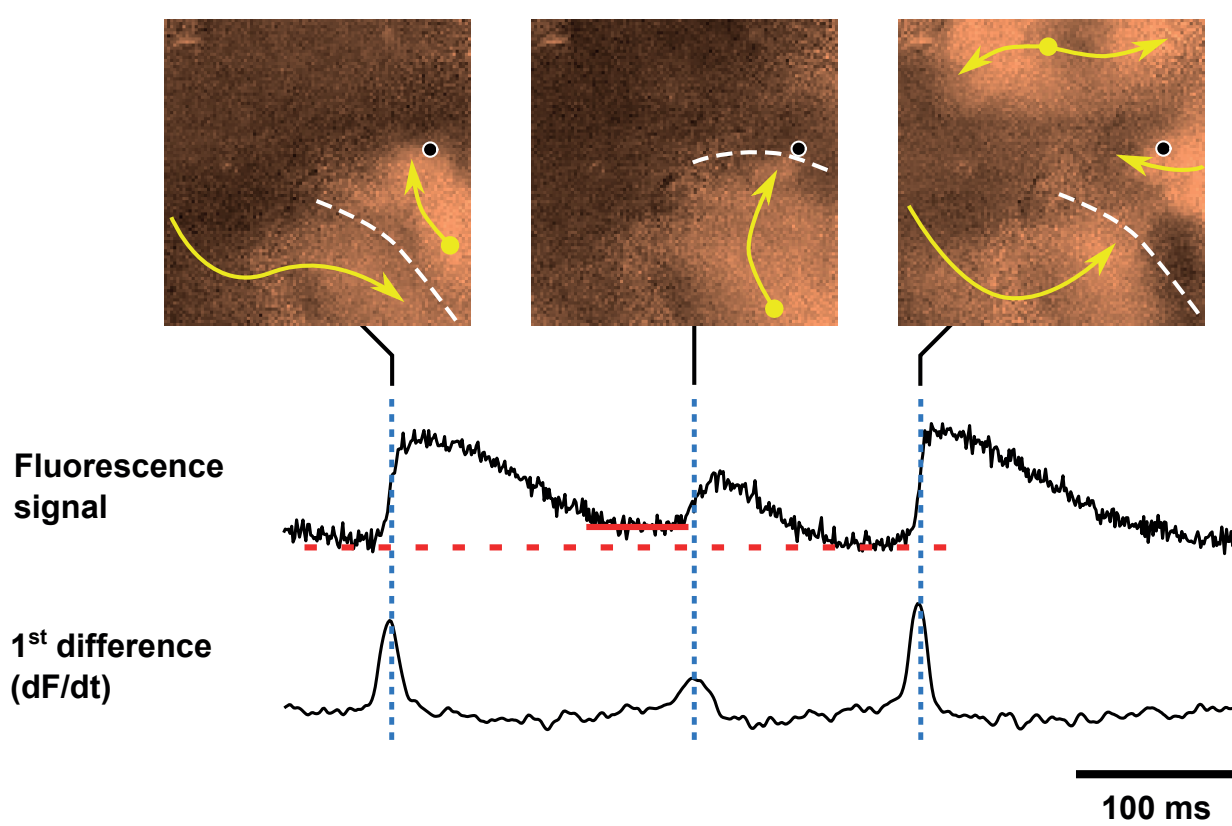

Figure 1. Variability of the optical action potential (OAP) shape and repolarization levels during atrial fibrillation (AF). Top: optical mapping images acquired during an AF episode in a perfused goat heart (AF maintained for 6 months). Multiple fibrillatory waves propagating through the mapped region are illustrated by yellow arrows. Middle: fluorescence signal of a single pixel (position within the mapped region indicated by a black circle) showing three OAPs with variable morphology. Bottom: $1^{\text {st }}$ difference of the fluorescence signal illustrating OAP upstroke. The time points when the optical mapping images were captured are marked by blue dashed lines. When an excitation wave propagated across the pixel, OAP exhibited high magnitude and fast upstroke, as observed in the first and the third OAP. However, in the proximity of the line of conduction block (white dashed line) both the magnitude and the upstroke of OAP decreased, as illustrated by the OAP in the middle. The OAP with aberrant morphology was preceded by an incomplete repolarization, as shown by the difference between the level of repolarization preceding the upstroke (solid red line) and the level of full repolarization (dashed red line). 


\section{METHODS}

\section{Animal model}

The study was approved by the local ethics committee for animal experimentation and complies with the European directive 2010/63/EU on the protection of animals used for scientific purposes. Twelve Dutch milk goats $(54 \pm 11 \mathrm{~kg}$, range $41-74 \mathrm{~kg})$ were anesthetized using sodium thiopental ( $20 \mathrm{mg} / \mathrm{kg}$ IV) for induction of anesthesia and sufentanyl $(6 \mu \mathrm{g} / \mathrm{kg} / \mathrm{h}$ IV) and midazolam $(0.8 \mathrm{mg} / \mathrm{kg} / \mathrm{h} \mathrm{IV})$ for its maintenance. A bipolar lead was inserted into the right atrium through the jugular vein and connected to a subcutaneously placed implantable pacemaker (Medtronic Itrel $^{\oplus}$, Medtronic, Minneapolis, Minnesota, USA). After a recovery period of 2-3 weeks, AF was induced and maintained by burst pacing either for 3 weeks (short term, ST; $\mathrm{n}=6$ ) or for 6 months (long term, LT; $\mathrm{n}=6$ ).

\section{Optical mapping}

At sacrifice under general anesthesia (see above), the chest was opened with a left-sided thoracotomy and hearts were excised and perfused with cold cardioplegic solution (concentrations in mM: $123 \mathrm{NaCl}, 15 \mathrm{KCl}, 25 \mathrm{NaHCO}_{3}, 1.3 \mathrm{KH}_{2} \mathrm{PO}_{4}, 0.5 \mathrm{MgSO}_{4} \cdot 7 \mathrm{H}_{2} \mathrm{O}$, 5.5 glucose and $2.0 \mathrm{CaCl}_{2} \cdot 2 \mathrm{H}_{2} \mathrm{O}$, saturated with $95 \% \mathrm{O}_{2}$ and $5 \% \mathrm{CO}_{2}$ ). The left and right coronary arteries were cannulated, the heart was transferred into a heated tissue chamber $\left(37^{\circ} \mathrm{C}\right)$ and perfused with modified Krebs-Henseleit solution (concentrations in mM: 118.5 $\mathrm{NaCl}, 3 \mathrm{KCl}, 25 \mathrm{NaHCO}_{3}, 1.2 \mathrm{KH}_{2} \mathrm{PO}_{4}, 1.2 \mathrm{MgSO}_{4} \cdot 7 \mathrm{H}_{2} \mathrm{O}, 11$ glucose and $1.2 \mathrm{CaCl}_{2} \cdot 2 \mathrm{H}_{2} \mathrm{O}$, saturated with $95 \% \mathrm{O}_{2}$ and $5 \% \mathrm{CO}_{2}$ ). Voltage-sensitive dye di-4-ANEPPS (amino-naphthylethylen-pyridium-propyl-sulfonate, Invitrogen, Carlsbad, California, USA and Eurogentec, Seraing, Belgium) was added to the solution together with excitation-contraction uncoupler blebbistatin $(10 \mu \mathrm{M}$, Bio-Connect B.V., Huissen, The Netherlands and Sigma-Aldrich, St. Louis, Missouri, USA). An area of approximately $2 \times 2 \mathrm{~cm}$ on the left atrial free wall was imaged with a 100x100 pixel Ultima-L CMOS camera (SciMedia, Costa Mesa, California, USA), resulting in a spatial resolution of approximately $200 \mu \mathrm{m}$. AF was initiated by burst pacing and optical mapping files of 1 to 4 seconds were recorded with frame rates ranging from 0.5 to $2 \mathrm{kHz}$.

\section{Data Analysis}

The data analysis method was implemented in MATLAB (The MathWorks, Inc., Natick, Massachusetts, USA) and can be described in the following steps:

Step 1: Signal normalization. Unequal amplitude of fluorescence signals within the mapped area caused by local differences in fluorescence intensity was corrected by signal normalization per trace. ${ }^{16}$ The image data from the Ultima-L CMOS camera were normalized such, that 
for each pixel the minimum fluorescence corresponded to 0 and peak fluorescence to $1 .{ }^{16} \mathrm{An}$ example of a single pixel fluorescence signal acquired during an episode of $\mathrm{AF}$ is shown in Figure 2A.

A

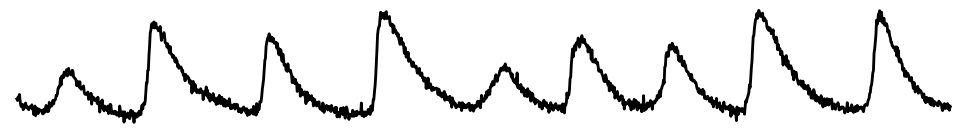

B
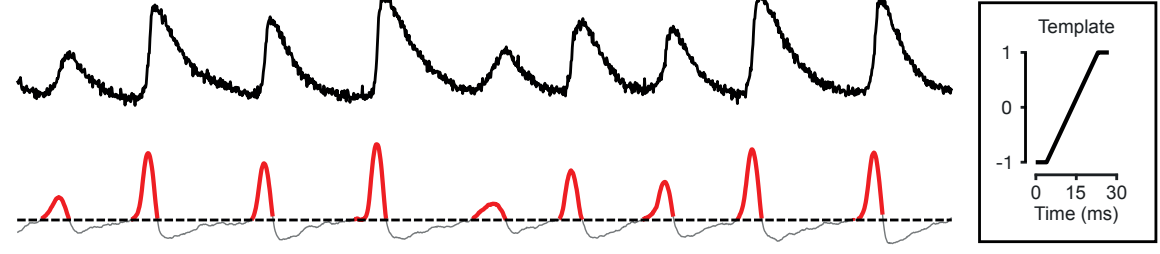

C

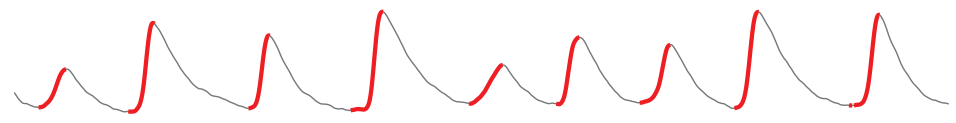

D

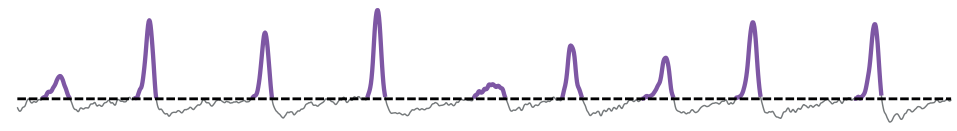

E

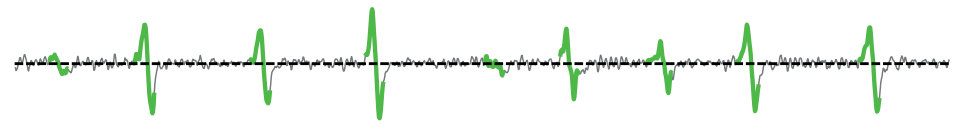

$\mathbf{F}$

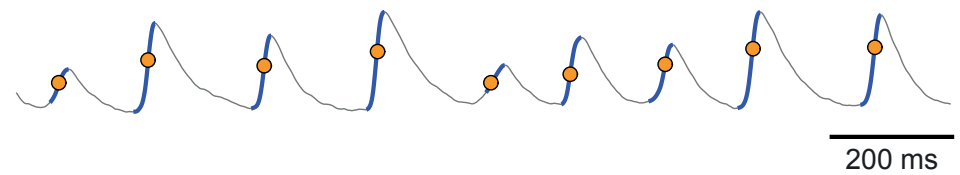

Figure 2. Detection of optical action potential (OAP) upstrokes in an optical mapping recording of atrial fibrillation (AF). (A) Example of a pixel signal acquired during an AF episode. (B) Cross-correlation of the pixel signal with the upstroke template (shown in the inset). The parts of the signal with positive values are highlighted in red. The dashed line in this panel and in panels (D) and (E) represents zero level. (C) Smoothened pixel signal with highlighted upstroke parts. The upstroke intervals correspond to the intervals with positive cross-correlation with the template. (D) First derivative of the smoothened signal (dF/dt). The intervals with $\mathrm{dF} / \mathrm{dt}>0$ are highlighted in purple. (E) Second derivative of the smoothened signal $\left(\mathrm{d}^{2} \mathrm{~F} / \mathrm{dt}^{2}\right)$ with the intervals corresponding to the upstrokes shown in green. (F) Based on the first and second derivative estimates, the upstroke parts of OAPs were identified (highlighted in blue) together with the points of maximum upstroke ( $\mathrm{dF} / \mathrm{dt}_{\text {max }}$ marked with orange circles).

Step 2: Identification of upstroke candidates. Candidate upstrokes were identified by sensitive template matching. A similar approach has been successfully implemented for detection of intrinsic deflections in atrial electrograms. ${ }^{22}$ A simple template was constructed, as shown in the inset in the Figure 2. A cross-correlation function of the pixel signal and the template was calculated (Figure $2 \mathrm{~B}$ ). To decrease the possibility of missing atrial activations with slow upstroke and low amplitude, all intervals with positive value of the cross-correlation 
were identified as possible candidates for action potential upstroke (Figure 2B, indicated in red). This approach was intentionally designed as overly sensitive and resulted in numerous false positive activations for each pixel. The candidate intervals for upstrokes projected on OAPs are illustrated in Figure 2C.

Step 3: Identification of upstrokes based on the first derivative of fluorescence. To reduce noise, the fluorescence signals were filtered using locally weighted scatterplot smoothing method with a span of 21 samples (Figure 2C). ${ }^{23}$ For each interval of positive cross-correlation that has been identified in step 2, the first fluorescence derivative $(\mathrm{dF} / \mathrm{dt})$ and second derivative $\left(\mathrm{d}^{2} \mathrm{~F} / \mathrm{dt}^{2}\right)$ estimates were calculated. The OAP upstroke was identified as the interval with positive first derivative $(d F / d t>0$, Figure $2 D)$ that contains exactly one inflection point $\left(d^{2} F /\right.$ $\mathrm{dt}^{2}=0$; Figure $2 \mathrm{E}$ ). The time points of maximum upstroke (maximum first derivative, $\mathrm{dF} / \mathrm{dt}_{\max }$ ) were considered as candidates for the moments of atrial activation (Figure 2F).

Step 4: Spatiotemporal filtering. The algorithm described above is prone to identify spurious activations due to noise as well as other small fluctuations in the fluorescence signal as potential OAP upstrokes. To eliminate false positive detections, we exploited the fact that true atrial activations that belong to the same excitation wave are grouped together in time and space and applied spatiotemporal filtering. A 3D space was constructed using the OAP upstroke intervals. A convolution with a $3 \mathrm{D}$ Gaussian kernel (size 3x3x15 samples, $\sigma_{\text {spatial }}=0.5, \sigma_{\text {temporal }}=1.75$ ) was performed. When the value of the convolution was lower than an empirically set threshold, the OAP upstrokes with their respective moments of activations were removed. The effect of the spatiotemporal filtering on atrial activations is demonstrated on examples of AF recordings shown in Figure 3. To quantify the effect of spatiotemporal filtering, AF recordings from the ST and LT goats were evaluated. Averaged per one second of recording, the number of initial activations was compared to the number of activations after spatiotemporal filtering using a paired t-test. Additionally, the percentage of activations removed by spatiotemporal filtering was compared between the ST and LT group using an unpaired t-test. The value of $\mathrm{p}<0.05$ was considered statistically significant.

To test the ability of the method to detect small fibrillatory waves, a comparison with a standard method based on thresholding of the first derivative of the optical mapping signal (dF/dt $t_{\max }>$ threshold) was used. ${ }^{14,16}$ To allow fair comparison of the two approaches, the same data preprocessing steps were used, including the signal filtering methods. 

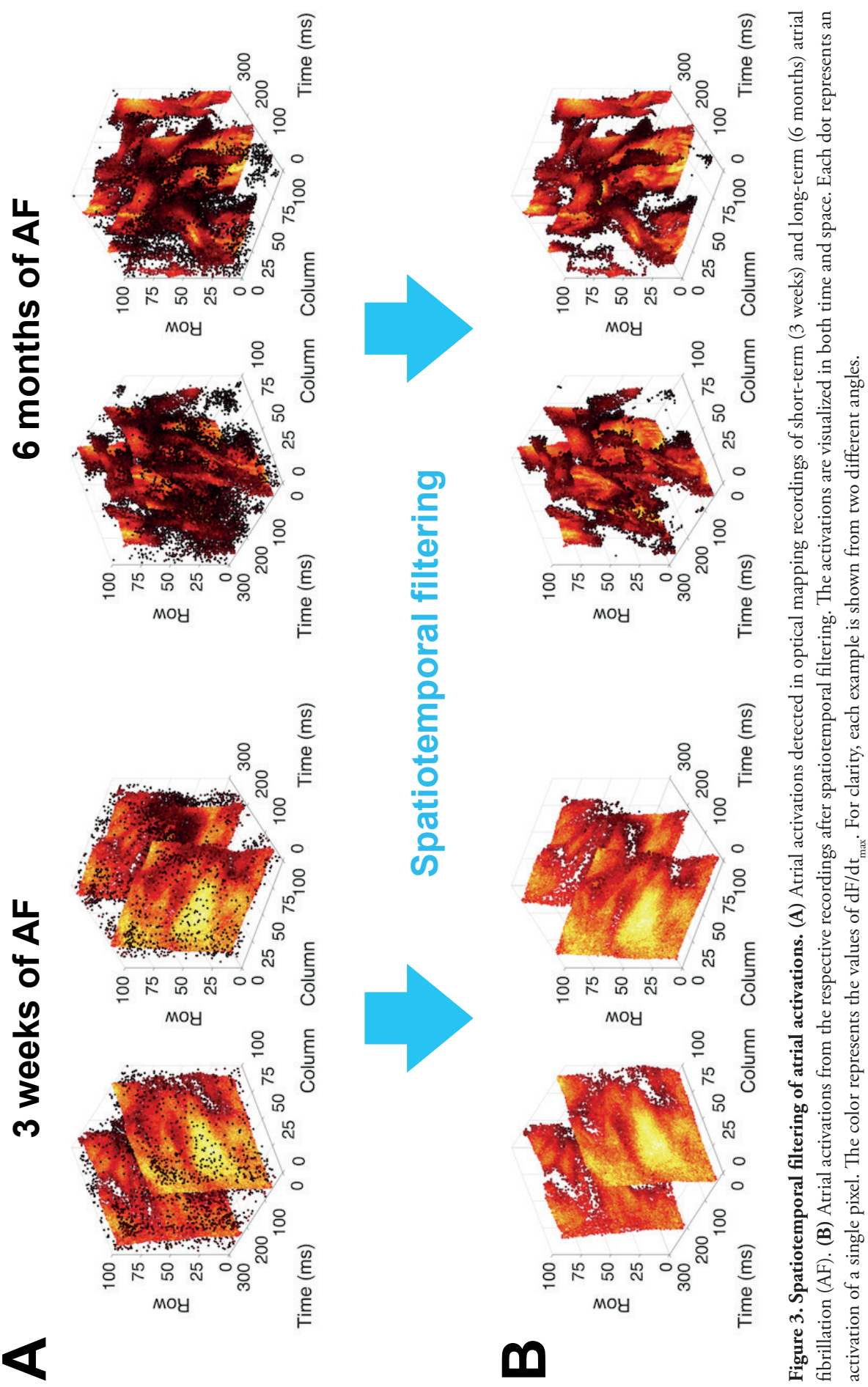


\section{RESULTS}

The presented method (referred to as 'propagation method') was applied on AF data acquired in the perfused hearts from goats with persistent $\mathrm{AF}$ and compared with a method based on

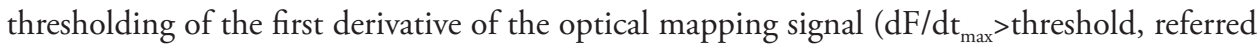
to as 'threshold method').

Illustrated on OAPs acquired during AF, Figure 4 shows similar performances of both methods to identify OAPs with fast upstrokes. When the OAP exhibited a slow upstroke, the propagation method was still capable to detect such OAP while the threshold method might leave this OAP undetected (OAPs indicated by red arrows in Figure 4E). The higher sensitivity of the propagation method to identify OAPs with slow upstroke is further illustrated in Figure 5A. The histograms acquired in a representative recording of AF show how often OAPs with a given steepness of upstroke $(\mathrm{dF} / \mathrm{dt})$ were observed. The threshold method shows a clear cut-off at $\mathrm{dF} / \mathrm{dt}=0.01$. The propagation method however was capable to identify OAPs with upstroke velocities below this threshold. At a lower threshold for steepness of the OAP upstroke, the threshold method had tendency to detect the same action potential multiple times, as shown in Figure 4E (purple arrow). In the example presented in Figure 5B, 5.9\% of all atrial activations identified by the threshold method were separated from the nearest activation by $<20$ milliseconds, indicating that these activations were very likely detected within the same OAP. On the other hand, only $0.02 \%$ of the activations identified by the propagation method occurred in the proximity of $<20$ milliseconds from the nearest activation.

To quantify the effect of spatiotemporal filtering, AF recordings acquired in the hearts of ST and LT goats were used. The application of spatiotemporal filtering on candidate activations resulted in reduction in the number of activations in both groups, as shown in Figure 6 . The percentage of activations removed by spatiotemporal filtering was comparable between the groups $(24.6 \pm 18.0 \%$ vs. $25.3 \pm 12.3 \%$, ST vs. LT, respectively; $\mathrm{p}=0.939)$, indicating no effect of AF complexity on the performance of spatiotemporal filtering.

To demonstrate how identification of OAPs affects detection of individual fibrillatory waves, we visually compared the waves detected by the propagation method with the waves that were detected by the threshold method. Combining template matching with spatiotemporal filtering, Figure 7 demonstrates that the propagation method was able to detect a small fibrillatory wave that was only partially detected by the threshold method. The example shows a wave, detected by the propagation method, that originated in the center of the mapped region. In the following time steps the territory of the wave progressively expanded. In contrast, the threshold method revealed only small parts of the wave, mainly at the edge of the wave detected by the propagation method. In the following time steps multiple isolated sites of activation appeared, 
reminiscent to noise. The difference in the performance of the two methods that is illustrated in Figure 7A occurred when the upstroke of the OAP was slow and its magnitude was low. This is illustrated by examples of OAPs acquired in two representative pixels (Figure 7C).
A
B
Original fluorescence signal
Filtered fluorescence signal

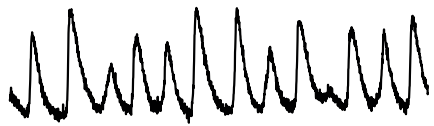

$500 \mathrm{~ms}$

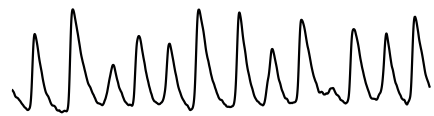

Propagation method

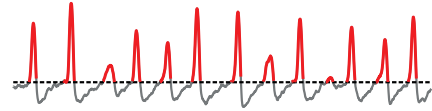

Threshold method

\section{Cross-correlation of the signal with the template
D $1^{\text {st }}$ difference
D of the filtered signal \\ E $\begin{aligned} & \text { Filtered signa } \\ & \text { with marked } \\ & \text { activations }\end{aligned}$}
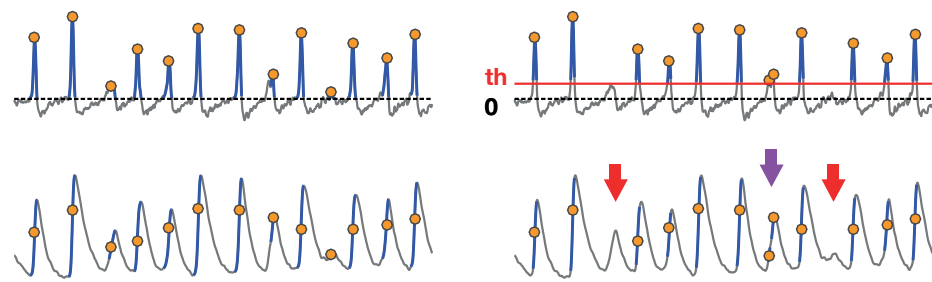

Figure 4. Comparison of optical action potential (OAP) upstroke detection between the propagation method and the threshold method. (A) Original pixel signal. (B) Filtered signal. (C) Cross-correlation of the original signal and the detection template (shown in the inset of Figure 2). (D) Detection of OAP upstrokes based on the first difference of the filtered signal. In the propagation method (left), parts of the signal with positive values of the crosscorrelation are selected and the intervals with positive $1^{\text {st }}$ difference (highlighted in blue) are identified. For each interval, only one maximum value ( $\mathrm{dF} / \mathrm{dt}_{\max }$, orange circles) is assigned. The dashed line represents zero level. In the threshold method (right), a dF/dt threshold is determined (solid red line) and the intervals of positive first difference are identified (highlighted in blue). (E) Comparison of the detection performance between the two methods shows that using the method solely based on $\mathrm{dF} / \mathrm{dt}$ thresholding (right), some of the upstrokes remain undetected (red arrows) or might be detected twice (purple arrow). 


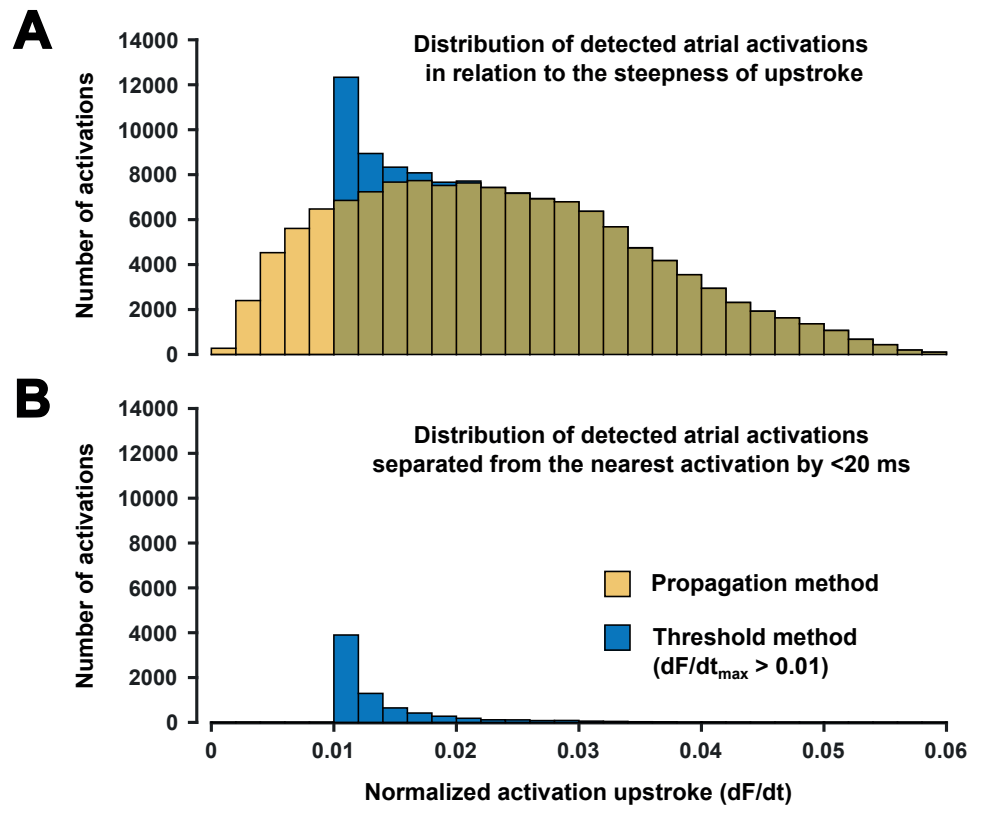

Figure 5. Performance of the propagation method and the threshold method in detection of optical action potential (OAP) upstrokes. The figure shows data from a representative optical mapping recording of AF that was acquired in a goat heart after 6 months of pacing-induced AF. (A) The distribution of detected atrial activations in relation to the steepness of the upstroke shows that the propagation method is able to identify OAP with upstrokes rates below the threshold that has been set for the threshold method $\left(\mathrm{dF} / \mathrm{dt}_{\max }<0.01\right)$. (B) The distribution of activations that are separated from the nearest activation by less than 20 milliseconds. These activations correspond to multiple detections of the same OAP upstroke.

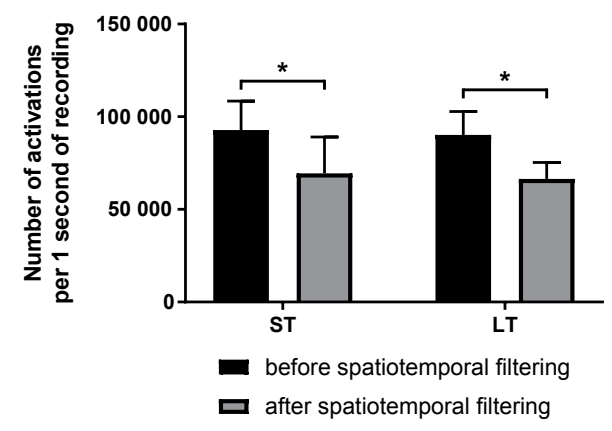

Figure 6. The effect of spatiotemporal filtering on the number of atrial activations. Spatiotemporal filtering reduced the number of atrial activations in the AF recordings acquired in the hearts from both short-term (ST) and long-term (LT) goats ( $\mathrm{p}=0.025$ and $\mathrm{p}=0.013$ for $\mathrm{ST}$ and LT group, respectively; $\mathrm{n}=6$ for each group). 
A

Aropagation
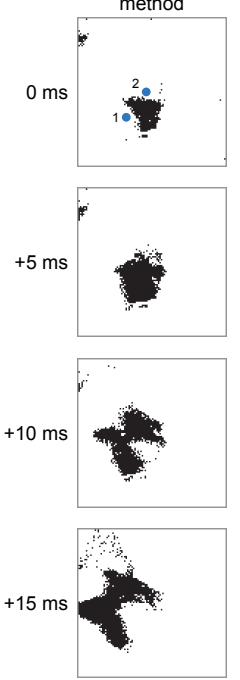
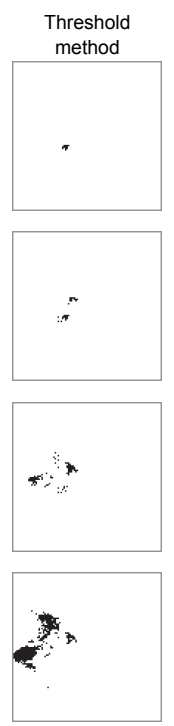

Difference
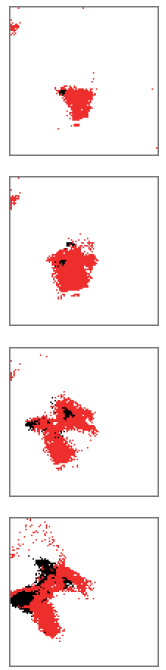

B

Propagation method

Threshold method

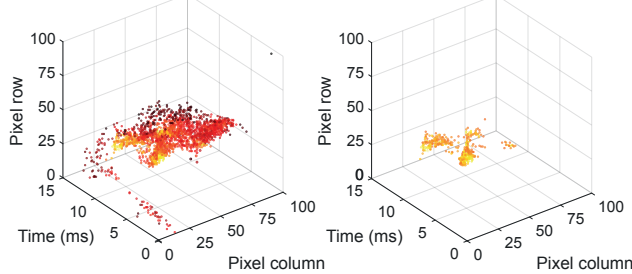

C

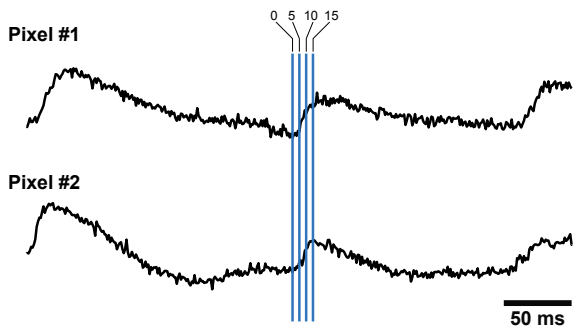

Figure 7. Comparison of wave detection between the propagation method and the threshold method. The example shows a wave that was detected by the propagation method but was detected only partially by the threshold method. (A) Spatial patterns showing atrial activations detected by the propagation method (left column) and by the threshold method (middle column). The right column demonstrates the differences between the methods' performance, with red pixels indicating the activations that were detected by the propagation method but not by the threshold method. Black pixels indicate activations that were detected by both methods. (B) Visualization of detected atrial activations in time and space. Each dot represents an activation of a single pixel. The color represents the values of $\mathrm{dF} / \mathrm{dt}_{\text {max }}$ (C) Examples of fluorescence signals of two selected pixels. The positions of the pixels are indicated in the top left example in the panel (A). The blue lines indicate the time points of the spatial patterns shown in the panel (A).

An even more remarkable difference in the performance of the two methods is demonstrated in Figure 8, clearly showing that the propagation method is capable of identifying small fibrillatory waves that remained undetected by the threshold method. Figure 8 shows an example of four small fibrillatory waves with various shapes that occurred in the mapped region. Three of them were already present in the first time step $(0 \mathrm{~ms})$. Within the visualized time window of 20 milliseconds, the line-shaped wave in the upper part of the mapped region propagated to the left, the wave at the right side of the field of view propagated towards the top and the wave at the bottom disappeared after $10 \mathrm{~ms}$. The fourth fibrillatory wave originated in the center of the mapped region within the first $5 \mathrm{~ms}$. None of the waves was detected by the threshold method, as illustrated by the panels A and B of Figure 8. Similarly to the excitation wave shown in Figure 7, also the fibrillatory waves presented in Figure 8 were characterized by OAPs exhibiting low amplitude and slow upstroke (Figure 8C). 

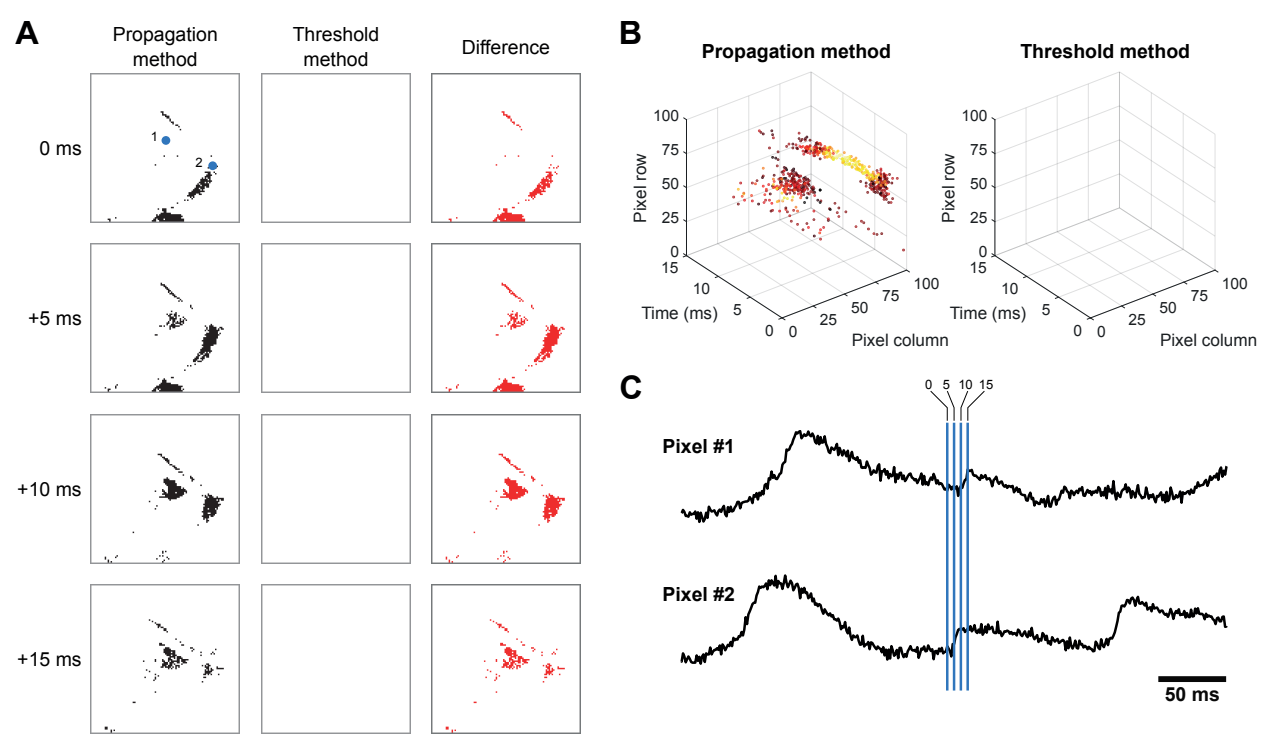

Figure 8. An example of fibrillatory waves that were detected by the propagation method but were not detected by the threshold method. The layout and organization of the figure is the same as in Figure 7.

\section{DISCUSSION}

This work presents a novel method for detection of atrial activations in optical mapping recordings of AF. The method is based on overly sensitive template matching, followed by the removal of false positive activations by spatiotemporal filtering, thereby incorporating the principle of wave propagation in a very simple and straightforward manner. We tested performance of the method using optical mapping recordings of AF that were acquired in explanted perfused hearts of goats that had been in AF for either 3 weeks or 6 months.

The main advantage of the presented method (propagation method) is its sensitivity to detect optical action potentials (OAPs) with aberrant morphologies that occur during slow, discontinuous conduction. Using highly sensitive template matching and identification of OAP upstrokes based on the first and the second derivative of the fluorescence signal, the propagation method identified OAPs that exhibit slow upstroke and low magnitude, as demonstrated in Figures 4 and 5. These OAPs frequently occur during complex discontinuous atrial conduction, as illustrated in Figure 1, and are also associated with occurrence of small fibrillatory waves. Being ensemble action potentials, the magnitude of OAPs that represent small fibrillatory 
waves is low, because the part of the tissue that is being activated at a time is small. In addition, having a small source, these waves propagate relatively slowly. As a consequence of that, these fibrillatory waves often exhibit low conduction velocity and therefore slow upstrokes.

When using the method that is solely based on thresholding of $\mathrm{dF} / \mathrm{dt}$ (threshold method), a non-zero threshold has to be applied to detect activations within a single trace. To achieve acceptable signal-to-noise ratio however, some OAPs may remain undetected, thus representing false negatives. Increasing the sensitivity of the method by lowering the threshold decreases the number of false negative detections but increases the number of false positive detections due to signal artefacts and residual noise. Moreover, OAP upstrokes exhibiting aberrant, fractionated shape can be detected more than just once when the threshold method is implemented without a period of blanking, as illustrated by the OAP under the purple arrow in Figure 4E. Initial detection of upstrokes by template matching that is followed by identification of $\mathrm{dF} / \mathrm{dt}_{\max }$ decreases the possibility that multiple atrial activations will be identified within a single upstroke, and thus increases robustness of the propagation method.

In contrast to the false positive detections of residual noise that occur randomly in time and space, the true atrial activations appear in close proximity because they form a propagating excitation wave. Moreover, as the excitation waves propagate with a certain conduction velocity, the OAPs occur in a successive order over time in adjacent pixels. As a result of this behavior, the true atrial activations are grouped together in both time and space. To detect aberrant OAPs that form small excitation waves, we utilized this behavior by applying spatiotemporal filtering. Filtering of the initially detected activations in both time and space separates the detections due to the residual noise and artefacts from the detections that correspond to a propagating wave. This property of the propagation method allows identification of small fibrillatory waves. These waves would have remained undetected or detected only in part if the OAPs were solely identified on strict thresholding of dF/dt, as demonstrated in Figure 7 and Figure 8.

The ability to detect small fibrillatory waves may have considerable implications for analysis of arrhythmic data and subsequent interpretation of the reconstructed activation patterns. Identifying large excitation waves while leaving small fibrillatory waves undetected may result in the perception that fibrillatory patterns appear simpler than they actually are. Similarly, partial detection of excitation waves may substantially alter the interpretation to which degree the excitation wavefronts are fractionated. Having a sensitive method for detection of atrial activations is therefore essential for analysis of activation patterns reconstructed from mapping data. Using contact electrograms, our group previously reported that fibrillation patterns become more complex with structural remodeling. ${ }^{8}$ However, due to the physical principle used, the spatiotemporal resolution of contact electrical mapping is inherently lower than 
that of optical mapping. Due to that, contact electrical mapping may not be able to depict full complexity of atrial activation during $\mathrm{AF}^{24}$ In this regard, optical mapping represents an invaluable tool for detailed characterization of atrial propagation patterns.

Several algorithms have been introduced for analysis of optical mapping recordings acquired during AF. However, these methods focused mainly on analysis in frequency domain ${ }^{16,19}$ to identify location of possible AF drivers. Another approach is represented by analysis in phase domain, which allows assessment of reentrant activity and rotors. ${ }^{20,21}$ In this study, we compared our method with a method based on thresholding of the first derivative of the OAP upstroke, which is the most common approach. ${ }^{25,26}$ Alternatively, the times of activation can be defined by depolarization mid-point, ${ }^{25,27}$ action potential peak or by using the second derivative $\left(\mathrm{d}^{2} \mathrm{~F} / \mathrm{dt}^{2}\right)$ of the action potential upstroke. ${ }^{17}$ Following the identification of upstroke intervals by cross-correlation with the upstroke template, we based the estimation of activation times on $\mathrm{dF} / \mathrm{dt}_{\max }$, since this method is widely used and known to provide reliable results. To illustrate the advantages of the propagation method, we used the comparison with the threshold method, as this method is from the signal processing perspective the most closely related and commonly used method available.

Compared to the template matching method that has been successfully applied for detection of intrinsic deflections in atrial electrograms, ${ }^{22}$ the presented method uses only one duration of the template, adjusted for the sampling frequency. The reason for implementing the template matching with only one template was mainly practical. During the development of the presented algorithms, only a minor increase in detection performance was observed when multiple templates of different duration were used for the cross-correlation with the optical mapping signal. This finding suggests that the cross-correlation of the signal with a single upstroke template is sufficient for successful detection of OAP upstrokes. Another aspect favoring the use of a single template over multiple templates was reduction of the computational time that was necessary for primary detection of upstrokes.

Preprocessing of optical mapping data that includes filtering in spatial and temporal domain and correction for baseline drift can substantially enhance the quality of the recordings. ${ }^{28,29}$ However, although there is a multitude of methods available, no widely accepted consensus regarding their use exists. ${ }^{16}$ A commonly used approach is to apply filtering in both spatial and temporal domain as a preprocessing step. ${ }^{16,17}$ We intentionally avoided spatial filtering to prevent occurrence of blurring that would make identification of small fibrillatory waves more difficult. Similarly, template matching was applied on optical mapping recordings before filtering in temporal domain to avoid the possibility that only OAPs with steep upstrokes and large magnitudes are detected. The implemented method is based on identification of 
upstrokes, which in general are phenomena exhibiting high-frequency changes. Therefore, correction for a baseline drift was not necessary as this is a preprocessing step that removes low frequencies from the signal.

\section{LIMITATIONS}

One of the major limitations of this work is absence of a comparison that would allow quantitative expression of the method's performance. As none of the previously published methods has been fully validated for beat-to-beat detection of OAPs in arrhythmic data, there is currently no approach that can be considered as gold standard. Moreover, evaluation of the performance of the propagation method on the level of fibrillatory waves would require introducing the concept of a wave. As we consider the presented method as the first step in a chain of optical mapping data processing, we wanted to avoid implementation of an algorithm for identification of fibrillatory waves. Therefore, we decided to demonstrate the advantages of the propagation method only on several examples, illustrating that the method performs well in detection of small fibrillatory waves in optical mapping recordings of AF.

\section{CONCLUSION AND FUTURE PERSPECTIVES}

A novel method for detection of action potentials in optical mapping recordings of AF has been developed, combining sensitive template matching for detection of action potential upstrokes with spatiotemporal filtering of atrial activations. The study demonstrates that the method is sensitive enough to identify OAPs with low magnitude and slow upstroke and is capable of detecting small fibrillatory waves. The presented method can be considered as a first step for further analysis of optical mapping recordings acquired during cardiac fibrillation, allowing advanced analysis of activation patterns and identification and tracking of excitation waves. ${ }^{30}$ Especially, it has a substantial potential to become a useful tool for studying discontinuous atrial conduction and zig-zag patterns, the phenomena that have been observed in structurally remodeled atria. ${ }^{18,31}$ 


\section{REFERENCES}

1. Bjorck S, Palaszewski B, Friberg L, Bergfeldt L. Atrial fibrillation, stroke risk, and warfarin therapy revisited: a population-based study. Stroke 2013;44:3103-3108.

2. Wolf PA, Abbott RD, Kannel WB. Atrial Fibrillation as an Independent Risk Factor for Stroke: The Framingham Study. Stroke 1991;22:983-988.

3. Stewart S, Hart CL, Hole DJ, McMurray J. A population-based study of the long-term risks associated with atrial fibrillation: 20-year follow-up of the Renfrew/Paisley Study. Am J Med 2002;113:359-364.

4. Kirchhof P, Benussi S, Kotecha D, Ahlsson A, Atar D, Casadei B, et al. 2016 ESC Guidelines for the management of atrial fibrillation developed in collaboration with EACTS. Europace 2016;18:16091678.

5. Schotten U, Verheule S, Kirchhof P, Goette A. Pathophysiological mechanisms of atrial fibrillation: a translational appraisal. Physiol Rev 2011;91:265-325.

6. Kopecky SL, Gersh BJ, McGoon MD, Whisnant JP, Holmes DR, Jr., Ilstrup DM, et al. The natural history of lone atrial fibrillation. A population-based study over three decades. $N$ Engl J Med 1987;317:669-674.

7. Spach MS, Josephson ME. Initiating reentry: the role of nonuniform anisotropy in small circuits. J Cardiovasc Electrophysiol 1994;5:182-209.

8. Verheule S, Tuyls E, van Hunnik A, Kuiper M, Schotten U, Allessie M. Fibrillatory conduction in the atrial free walls of goats in persistent and permanent atrial fibrillation. Circ Arrhythm Electrophysiol 2010;3:590-599.

9. Verheule S, Tuyls E, Gharaviri A, Hulsmans S, van Hunnik A, Kuiper M, et al. Loss of continuity in the thin epicardial layer because of endomysial fibrosis increases the complexity of atrial fibrillatory conduction. Circ Arrhythm Electrophysiol 2013;6:202-211.

10. Mandapati R, Skanes A, Chen J, Berenfeld O, Jalife J. Stable Microreentrant Sourcees as a Mechanism of Atrial Fibrillation in the Isolated Sheep Heart. Circulation 2000;101:194-199.

11. Verheule S, Wilson E, Banthia S, Everett TH, Shanbhag S, Sih HJ, et al. Direction-dependent conduction abnormalities in a canine model of atrial fibrillation due to chronic atrial dilatation. $\mathrm{Am}$ J Physiol Heart Circ Physiol 2004;287:H634-H644.

12. Hansen BJ, Zhao J, Csepe TA, Moore BT, Li N, Jayne LA, et al. Atrial fibrillation driven by microanatomic intramural re-entry revealed by simultaneous sub-epicardial and sub-endocardial optical mapping in explanted human hearts. Eur Heart J 2015;36:2390-2401.

13. Herron TJ, Lee P, Jalife J. Optical imaging of voltage and calcium in cardiac cells \& tissues. Circ Res 2012;110:609-623.

14. Efimov IR, Huang DT, Rendt JM, Salama G. Optical mapping of repolarization and refractoriness from intact hearts. Circulation 1994;90:1469-1480.

15. Berenfeld O, Efimov IR. Optical Mapping. Card Electrophysiol Cin 2019;11:495-510.

16. Laughner JI, Ng FS, Sulkin MS, Arthur RM, Efimov IR. Processing and analysis of cardiac optical mapping data obtained with potentiometric dyes. Am J Physiol Heart Circ Physiol 2012;303:H753765. 
17. O'Shea C, Holmes AP, Yu TY, Winter J, Wells SP, Correia J, et al. ElectroMap: High-throughput open-source software for analysis and mapping of cardiac electrophysiology. Sci Rep 2019;9:1389.

18. Koura T, Hara M, Takeuchi S, Ota K, Okada Y, Miyoshi S, et al. Anisotropic conduction properties in canine atria analyzed by high-resolution optical mapping: preferential direction of conduction block changes from longitudinal to transverse with increasing age. Circulation 2002;105:20922098.

19. Skanes AC, Mandapati R, Berenfeld O, Davidenko JM, Jalife J. Spatiotemporal periodicity during atrial fibrillation in the isolated sheep heart. Circulation 1998;98:1236-1248.

20. Chen J, Mandapati R, Berenfeld O, Skanes AC, Gray RA, Jalife J. Dynamics of wavelets and their role in atrial fibrillation in the isolated sheep heart. Cardiovasc Res 2000;48:220-232.

21. Filgueiras-Rama D, Price NF, Martins RP, Yamazaki M, Avula UM, Kaur K, et al. Long-term frequency gradients during persistent atrial fibrillation in sheep are associated with stable sources in the left atrium. Circ Arrhythm Electrophysiol 2012;5:1160-1167.

22. Zeemering S, Maesen B, Nijs J, Lau DH, Granier M, Verheule S, et al. Automated quantification of atrial fibrillation complexity by probabilistic electrogram analysis and fibrillation wave reconstruction. Conf Proc IEEE Eng Med Biol Soc 2012;2012:6357-6360.

23. Cleveland WS, Devlin SJ. Locally Weighted Regression: An Approach to Regression Analysis by Local Fitting. Journal of the American Statistical Association 1988;83:596-610.

24. King B, Porta-Sánchez A, Massé S, Zamiri N, Balasundaram K, Kusha M, et al. Effect of spatial resolution and filtering on mapping cardiac fibrillation. Heart Rhythm 2017;14:608-615.

25. Fast VG, Kléber AG. Cardiac tissue geometry as a determinant of unidirectional conduction block: assessment of microscopic excitation spread by optical mapping in patterned cell cultures and in a computer model. Cardiovasc Res 1995;29:697-707.

26. Efimov IR, Nikolski VP, Salama G. Optical Imaging of the Heart. Circ Res 2004;95:21-33.

27. Winter J, Bishop MJ, Wilder CDE, O’Shea C, Pavlovic D, Shattock MJ. Sympathetic Nervous Regulation of Calcium and Action Potential Alternans in the Intact Heart. Front Physiol 2018;9:16.

28. Mironov SF, Vetter FJ, Pertsov AM. Fluorescence imaging of cardiac propagation: spectral properties and filtering of optical action potentials. Am J Physiol Heart Circ Physiol 2006;291:H327-H335.

29. Sung D, Cosman JSJP, Mills R, McCulloch AD. Phase shifting prior to spatial filtering enhances optical recordings of cardiac action potential propagation. Ann Biomed Eng 2001;29:854-861.

30. Tomek J, Burton RAB, Bub G. Ccoffinn: Automated Wave Tracking in Cultured Cardiac Monolayers. Biophys J 2016;111:1595-1599.

31. Spach MS, Boineau JP. Microfibrosis produces electrical load variations due to loss of side-toside cell connections: a major mechanism of structural heart disease arrhythmias. Pacing Clin Electrophysiol 1997;20:397-413. 



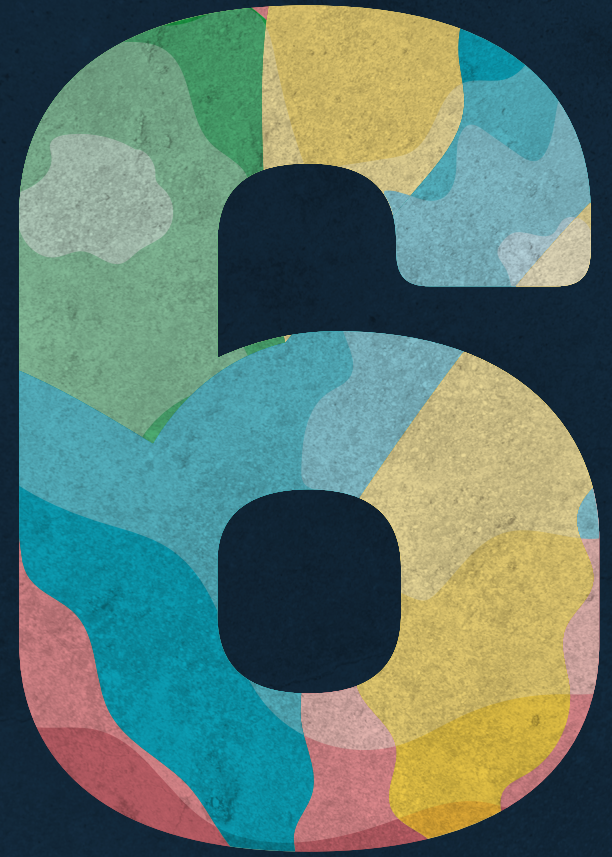




\section{Atrial repolarization pattern is more similar to the preceding activation pattern during atrial fibrillation than during atrial pacing}

Vladimír Sobota, Marion Kuiper, Arne van Hunnik, Nico H. Kuijpers, Ulrich Schotten and Sander Verheule

In preparation 


\section{ABSTRACT}

Background: Electrotonic interactions during atrial repolarization have not been studied extensively. This study aims to investigate the interaction of activation and repolarization patterns in the left atrium (LA) during pacing and atrial fibrillation (AF).

Methods: Optical mapping was performed in six perfused hearts of goats with 3 weeks of pacing-induced AF. Optical action potentials (100x100 pixels) were acquired in a $2 \times 2 \mathrm{~cm}$ area of the LA free wall during pacing (cycle length $350 \mathrm{~ms}$, 3-12 pacing sites per preparation) and AF. Activation time (AT, maximum upstroke derivative) and repolarization time (RT, time at $80 \%$ repolarization) were determined for each pixel. To assess the correlation between activation and repolarization patterns, we calculated the Pearson's correlation coefficient (r) between AT and RT. To evaluate the changes in dispersion of $\mathrm{APD}_{80}$ during pacing, we calculated the prolongation of $\mathrm{APD}_{80}\left(\triangle \mathrm{APD}_{80}\right)$ for each pixel as a difference between average $\mathrm{APD}_{80}$ derived from multiple pacing sites and the $\mathrm{APD}_{80}$ obtained during pacing at a particular site.

Results: Prolongation of $\mathrm{APD}_{80}$ at the site of pacing was observed $\left(\triangle \mathrm{APD}_{80} 8 \pm 5 \mathrm{~ms}, 6.6 \pm 4.2 \%\right)$, leading to a repolarization pattern with a weak correlation to the previous activation pattern $(r=0.22 \pm 0.24)$. During AF the correlation between AT and RT was stronger than during pacing $(\mathrm{r}=0.65 \pm 0.05)$.

Conclusion: During pacing, the action potential duration at the site of pacing prolonged, leading to rather synchronous repolarization within the field of view. By contrast, the repolarization during AF showed a wave-like propagating behavior, with pattern similar to the preceding activation. 


\section{INTRODUCTION}

The activation patterns in the atria and ventricles are well described. ${ }^{1,2}$ However, much less is known about their repolarization patterns. The repolarization sequence of human ventricles has been characterized relatively recently, ${ }^{3,4}$ showing evidence that the dispersion of ventricular repolarization is associated with occurrence of arrhythmias. ${ }^{5}$ Several studies also described that increased dispersion of atrial repolarization is associated with atrial fibrillation (AF) ${ }^{6,7}$

The analysis of atrial repolarization from contact electrograms still remains technically challenging, ${ }^{8,9}$ mainly because the atrial repolarization wave has a low amplitude and a gradual time course. ${ }^{10}$ The QRS complex may also obscure the atrial repolarization in electrograms. ${ }^{11}$ To date, there are only few in vivo studies on atrial repolarization, mainly limited to the use of monophasic action potentials $s^{6,7,12}$ or isolated atrial cardiomyocytes. ${ }^{13,14}$ Although the assessment of atrial repolarization from bipolar electrograms is technically feasible ${ }^{15}$ this method still requires further validation. ${ }^{16}$

Optical mapping with voltage-sensitive dyes is a well-established technique that has the great advantage of capturing optical action potentials with high spatial and temporal resolution ${ }^{17}$ and thus allows analysis of local repolarization patterns. However, studies on atrial repolarization patterns using this technique are still relatively scarce, focusing mainly on spatial dispersion of action potential duration or phase singularities. ${ }^{18-20}$ The present study aims to describe local activation and repolarization patterns in the left atrium (LA) during atrial pacing and AF and to describe the relationship between local activation and repolarization times. To study this interaction in the conditions that resemble clinically observed proarrhythmic changes in atria, ${ }^{21}$ we performed optical mapping in perfused preparations of goat hearts with a history of pacing-induced AF. These hearts recapitulate the AF-induced atrial remodeling that has been observed in AF patients. ${ }^{22}$

\section{METHODS}

\section{Animal model}

The animal study was approved by the local ethics committee for animal experimentation and complies with the European directive 2010/63/EU on the protection of animals used for scientific purposes. Six Dutch milk goats (body weight $50 \pm 8 \mathrm{~kg}$, range $41-64 \mathrm{~kg}$ ) were included. Anesthesia was induced with sodium thiopental $(20 \mathrm{mg} / \mathrm{kg} \mathrm{IV})$ and maintained with sufentanyl $(6 \mu \mathrm{g} / \mathrm{kg} / \mathrm{h} \mathrm{IV})$ and midazolam $(0.8 \mathrm{mg} / \mathrm{kg} / \mathrm{h} \mathrm{IV})$. A bipolar screw-in J-lead (Medtronic, Minneapolis, Minnesota, USA) was inserted through the jugular vein into the right atrium and connected to a subcutaneously implanted pacemaker (Medtronic Itrel ${ }^{\oplus}$, 
Medtronic, Minneapolis, Minnesota, USA). Following a recovery period of 2-3 weeks, AF was induced and maintained by applying a one-second burst of $50 \mathrm{~Hz}$ every other second for 3 weeks.

\section{Optical mapping}

Hearts were rapidly excised under anesthesia and perfused through the aorta with cold cardioplegic solution (concentrations in mM: $123 \mathrm{NaCl}, 15 \mathrm{KCl}, 25 \mathrm{NaHCO}_{3}, 1.3 \mathrm{KH}_{2} \mathrm{PO}_{4}$, $0.5 \mathrm{MgSO}_{4} \cdot 7 \mathrm{H}_{2} \mathrm{O}, 5.5$ glucose and $2.0 \mathrm{CaCl}_{2} \cdot 2 \mathrm{H}_{2} \mathrm{O}$, saturated with $95 \% \mathrm{O}_{2}$ and $5 \% \mathrm{CO}_{2}$ ). After cannulation of coronary arteries, the heart was transferred into a heated tissue chamber $\left(37^{\circ} \mathrm{C}\right)$ and perfused with modified Krebs-Henseleit solution (concentrations in mM: 118.5 $\mathrm{NaCl}, 3 \mathrm{KCl}, 25 \mathrm{NaHCO}_{3}, 1.2 \mathrm{KH}_{2} \mathrm{PO}_{4}, 1.2 \mathrm{MgSO}_{4} \cdot 7 \mathrm{H}_{2} \mathrm{O}, 11$ glucose and $1.2 \mathrm{CaCl}_{2} \cdot 2 \mathrm{H}_{2} \mathrm{O}$, saturated with $95 \% \mathrm{O}_{2}$ and $5 \% \mathrm{CO}_{2}$ ) at a perfusion pressure of $100-120 \mathrm{cmH}_{2} \mathrm{O}$. The voltagesensitive dye di-4-ANEPPS (amino-naphthyl-ethylen-pyridium-propyl-sulfonate, Invitrogen, Carlsbad, California, USA and Eurogentec, Seraing, Belgium) was added to the solution together with excitation-contraction uncoupler blebbistatin $(10 \mu \mathrm{M}$, Bio-Connect B.V., Huissen, The Netherlands and Sigma-Aldrich, St. Louis, Missouri, USA). The LA free wall was illuminated with Luxeon-I power LEDs $(505 \mathrm{~nm}$, Philips LumiLEDs) and an area of approximately $2 \times 2 \mathrm{~cm}$ was imaged with a 100x100 pixel Ultima-L CMOS camera (SciMedia, Costa Mesa, California, USA), resulting in a spatial resolution of approximately $200 \mu \mathrm{m}$. Various sites (3-12) within the field of view were stimulated with a unipolar silver electrode, applying the current of $1.1 x$ pacing threshold and a basic cycle length of $350 \mathrm{~ms}$. Imaging files were recorded with a frame rate of $2 \mathrm{kHz}$. Consequently, the heart was stimulated into AF and imaging files were recorded with a frame rate of $0.5-1 \mathrm{kHz}$.

\section{Computer simulation of atrial pacing}

In addition to optical mapping, we used a mathematical model of atrial tissue ${ }^{23,24}$ to study the effect of pacing on local changes in action potential duration (APD). The model was described by a monolayer consisting of 180 by 180 segments of size 0.01 by $0.01 \mathrm{~cm}$. The atrial membrane behavior of the segments was based on the Courtemanche-Ramirez-Nattel model of human atrial electrophysiology. ${ }^{25}$ To incorporate the anisotropy in epicardial conduction caused by muscle fiber orientation, longitudinal and transverse conductivities were set to $1.0 \mathrm{mS} / \mathrm{cm}$ and $0.12 \mathrm{mS} / \mathrm{cm}$, respectively. ${ }^{23}$ Local activation and repolarization times were defined as the times when the membrane potential increased above or decreased below an excitation threshold of $-60 \mathrm{mV}$, respectively. ${ }^{24}$ 


\section{Data Analysis}

The analysis of optical mapping data of atrial pacing was performed in a custom-developed software. ${ }^{24}$ To remove high-frequency noise, the data were down-sampled in time by a factor of two. Baseline drift was corrected by an iterative polynomial fitting algorithm. The analysis of optical mapping data acquired during AF was performed by using a custom-build algorithm that is described more in depth below. The optical mapping data analyses were performed in MATLAB programming environment (The MathWorks, Inc., Natick, Massachusetts, USA).

The method for estimating APD during AF further extends the algorithm for detection of atrial activations that has been described in chapter $\mathbf{5}$ and is presented in Figure 1. After identification of atrial activations by sensitive template matching and removal of false positive detections by spatiotemporal filtering, the signals were filtered by using locally weighted scatterplot smoothing with a span of 15 samples. Drift was identified by a second-degree polynomial fit through the local minima between the action potentials and subtracted from the raw signal. ${ }^{17}$

In all optical mapping recordings, activation time (AT) was determined for each action potential as the moment of maximum upstroke $\left(\mathrm{dF} / \mathrm{dt}_{\max }\right)$. The repolarization time (RT) was determined as the moment when fluorescence decreased below $80 \%$ of the action potential magnitude. Hereby, isochronal maps of AT and RT were constructed for recordings of pacing and AF. For each pacing site, one representative beat was recorded at a high frame rate to obtain a high temporal resolution. For the purpose of data analysis, the site of pacing was defined as the region that has been activated within the first 3 milliseconds (AT $\leq 3 \mathrm{~ms}$ ).

The dependency of the repolarization pattern (isochronal RT map) on the preceding activation pattern (isochronal AT map) was quantified by Pearson's correlation coefficient ( $r$ ) and by the slope of simple linear regression. Next, we studied the local changes in action potential duration at $80 \%$ of repolarization $\left(\mathrm{APD}_{80}\right)$ in relation to the pacing site. $\mathrm{APD}_{80}$ was calculated as a difference between $A T$ and RT. Per pixel, a reference $\mathrm{APD}_{80}$ was determined as the average $A P D_{80}$ from all pacing sites. Local changes in $\mathrm{APD}_{80}\left(\triangle \mathrm{APD}_{80}\right)$ were then calculated as the differences between local $\mathrm{APD}_{80}$ of the specific pacing site and the average $\mathrm{APD}_{80}$. The values were averaged per animal and the differences compared with paired t-test, considering $\mathrm{p}<0.05$ as statistically significant. For $\mathrm{AF}$ files, $\mathrm{APD}_{80}$ was determined as the difference between $\mathrm{AT}$ and RT as well. However, since full level of repolarization is usually not reached during AF, the values of $\mathrm{APD}_{80}$ obtained during $\mathrm{AF}$ should be considered as lower estimates of $\mathrm{APD}_{80}$. 


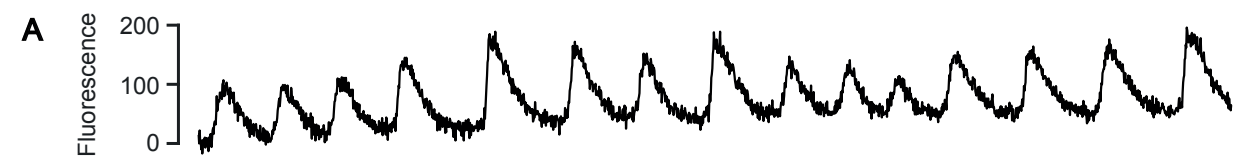

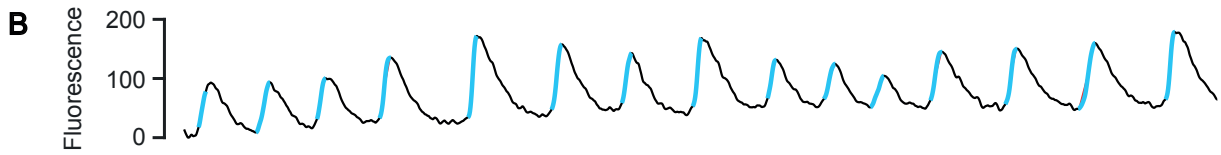

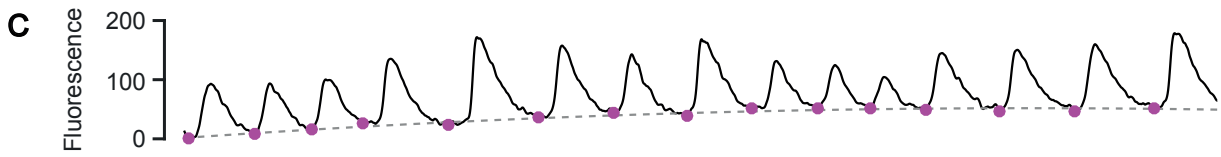

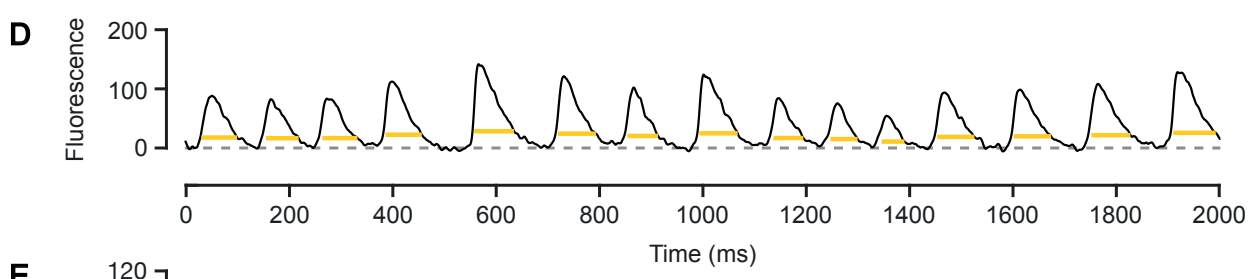

E

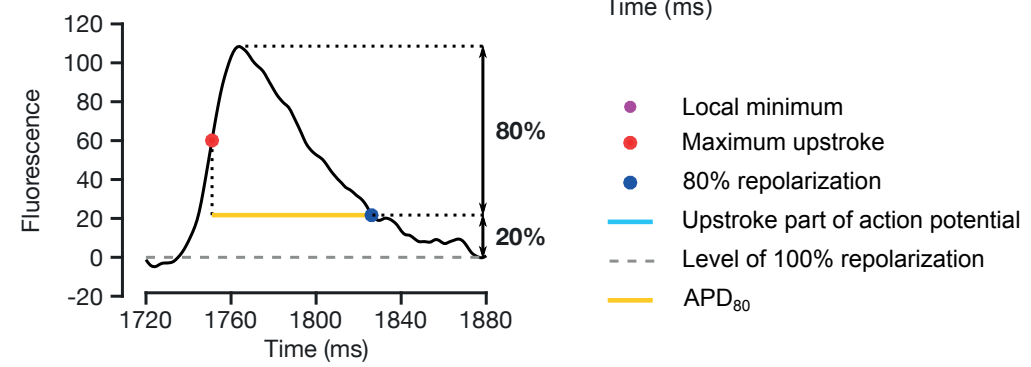

Figure 1. Analysis of action potential duration from optical mapping signals of atrial fibrillation. (A) Optical mapping signal of a single pixel. (B) Denoised signal with detected upstroke parts of optical action potentials. (C) Removal of baseline drift by interpolation of local minimum points by a second-degree polynomial. (D) Calculation of action potential duration at $80 \%$ repolarization $\left(\mathrm{APD}_{80}\right)$ for individual action potentials. (E) A detail of the analysis on a representative example of action potential. $\mathrm{APD}_{80}$ was calculated as the difference between the activation time (AT, the time of maximum upstroke, red circle) and repolarization time (RT, the time when fluorescence decreases below $80 \%$ of the action potential magnitude, blue circle).

\section{RESULTS}

Optical mapping files were recorded in 6 perfused goat hearts. Atrial stimulation was performed at $7 \pm 5$ (range 3-12) different pacing sites within the recording location. Data from $14 \pm 7$ (range 3-20) activation cycles during AF were analyzed per recording location. 


\section{Association between activation and repolarization patterns during pacing}

Representative examples of activation and repolarization patterns during atrial pacing are provided in Figure 2. Stimulation at different pacing sites within the field of view resulted in depolarization waves rapidly propagating through the atrial tissue, as shown by the activation patterns in Figure 2. The subsequent repolarization pattern was rather synchronous within the field of view, resulting in very similar repolarization patterns for different pacing locations. Accordingly, the maps of $\mathrm{APD}_{80}$ showed similar patterns for different pacing sites. However, a local prolongation of $\mathrm{APD}_{80}$ at the site of stimulation was frequently observed. To further investigate this phenomenon, graphs of the dependency of $\mathrm{APD}_{80}$ on the activation time were plotted, overall showing negative relationship between the activation time and $\mathrm{APD}_{80}$ (Figure 2). On average, $\triangle \mathrm{APD}_{80}$ at the site of pacing was prolonged by $8 \pm 5 \mathrm{~ms}(6.6 \pm 4.2 \%)$.

The effect of APD prolongation at the site of pacing was also studied in the computational model of atrial tissue, as shown in Figure 3. The activation pattern in the model (Figure 3A) was largely determined by the implemented tissue anisotropy. The activation time gradient was steeper in the horizontal direction, which is the direction that corresponds to the transversal orientation of muscle fibers, and was characterized by lower electrical conductivity. Spatial distribution of APD depicted in Figure 3B showed local prolongation of APD at the site of pacing. Besides that, the spatial distribution of APD was rather homogenous, with steeper gradient of APD decrease in the longitudinal direction (the direction with higher electrical conductivity) and pronounced APD shortening proximally towards the borders of the model.

\section{Association between activation and repolarization patterns during AF}

Examples of activation and repolarization patterns of three representative fibrillatory waves are shown in Figure 4. The activation patterns depict propagation of the depolarization wavefronts through the atrial tissue. In general, repolarization waves spread through the atrial tissue in a direction that was similar to the preceding activation waves, showing a similar speed of propagation (see the spacing of the isochronal lines of the repolarization patterns in Figure 2 for comparison). The repolarization patterns of the fibrillatory waves therefore resembled the preceding activation patterns. The maps of $\mathrm{APD}_{80}$ illustrating the difference between local activation and repolarization times show a spatially homogenous distribution within the field of view. This phenomenon is also demonstrated by the graphs in Figure 4, showing only a limited dependency of $\mathrm{APD}_{80}$ on activation time.

To assess the degree of similarity between activation and repolarization patterns, linear correlation between activation and repolarization times was quantified by Pearson's correlation coefficient. Positive as well as negative correlations between the activation and repolarization 
times were found during atrial pacing, as illustrated in Figure 5A. In contrast, only positive correlations were found between the activation and repolarization times during AF. When averaged per individual perfused preparation, a wide range of Pearson's correlation coefficient values was observed for atrial pacing, ranging from negative to positive values, but only a narrow range of positive correlations was observed for AF, as shown in Figure 5B. Similar findings were observed for the slope of linear regression that was calculated for the dependency of activation and repolarization times, as shown in Figure 5C. Overall, the results indicate that the degree of linear correlation between the activation and repolarization times during AF was higher than during atrial pacing.
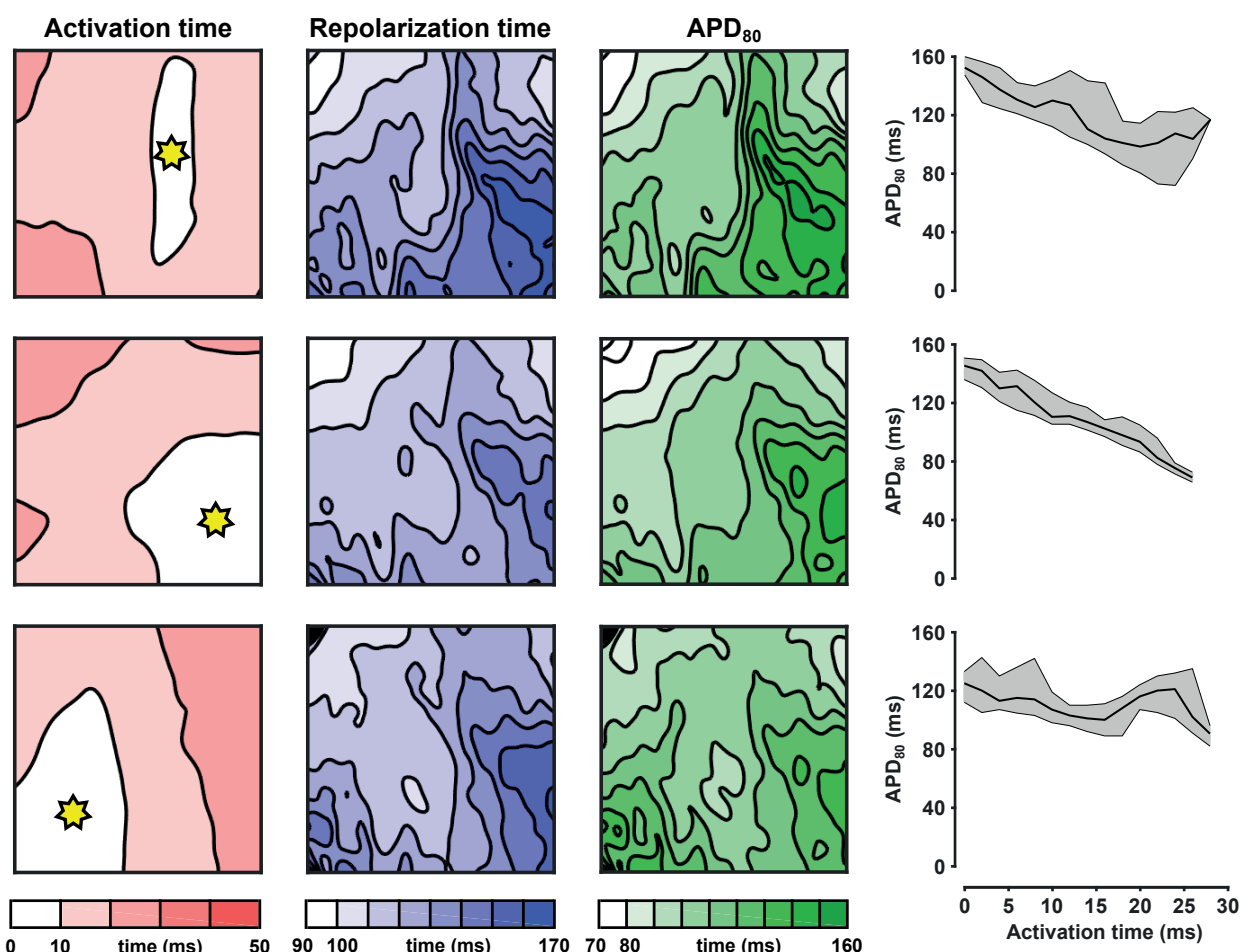

Figure 2. Activation patterns, repolarization patterns, $\mathrm{APD}_{80}$ distribution and the dependency of $\mathrm{APD}_{80}$ on activation time during local pacing in LA free wall. Each row represents a different stimulation site (indicated by a star in the activation map). The activation and repolarization patterns as well as the $\mathrm{APD}_{80}$ distribution are represented by isochronal maps with a step of $10 \mathrm{~ms}$. The graphs in the right column show the dependency of $\mathrm{APD}_{80}$ on activation time; the thick black line represents median with the grey area representing the range between the $10^{\text {th }}$ and $90^{\text {th }}$ percentile. 
A

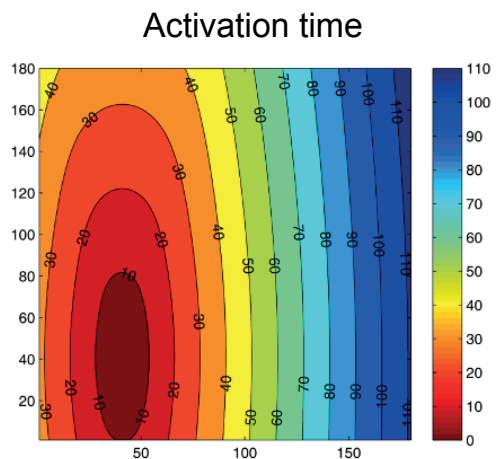

B

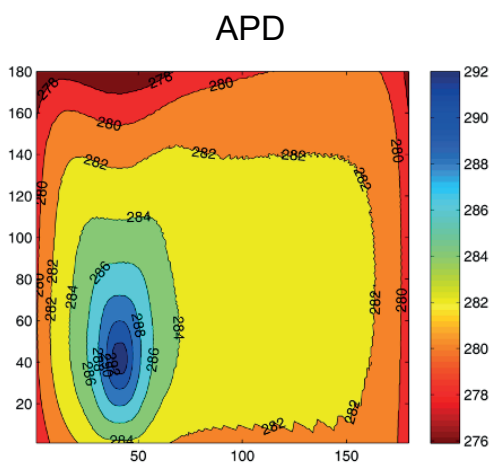

Figure 3. Computer simulation of atrial pacing in the monolayer of atrial cells. (A) Activation pattern during atrial pacing. The spatial distribution of activation times is given by the anisotropy of the model, implemented as different conductivities in horizontal and vertical direction. (B) Spatial distribution of action potential duration (APD). The model clearly shows local prolongation of APD at the site of stimulation.

Activation time
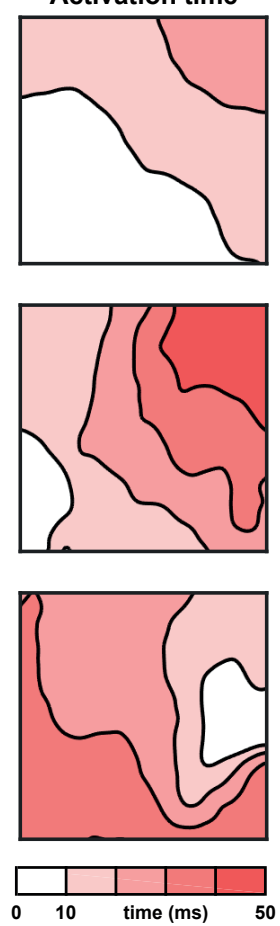
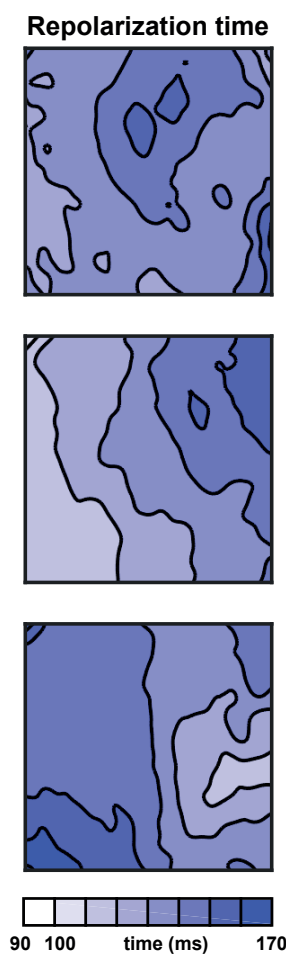
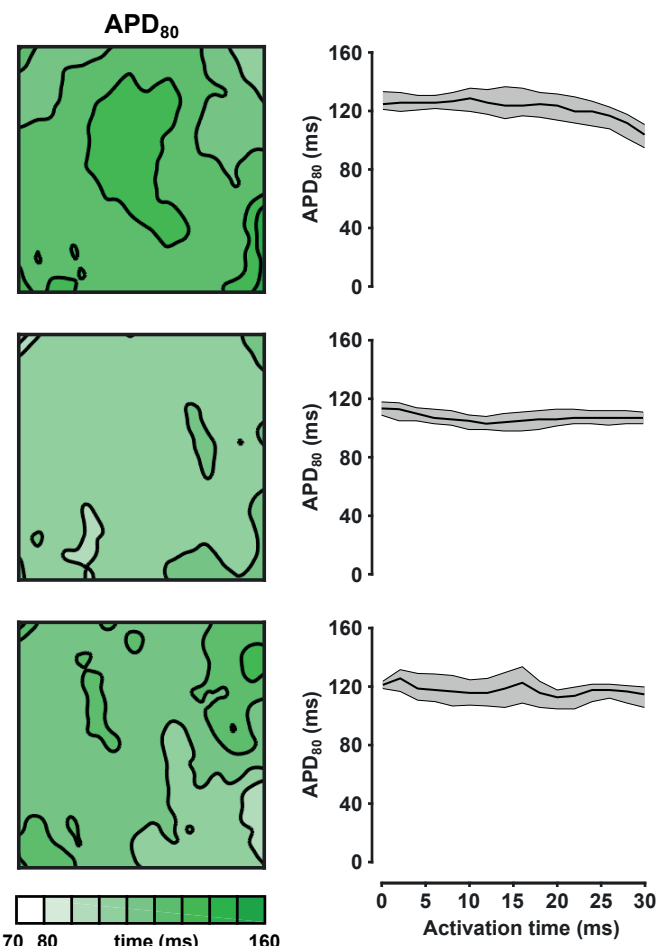

Figure 4. Activation patterns, repolarization patterns, $A P D_{80}$ distribution and the dependency of $A_{P D}$ on activation time during atrial fibrillation in LA free wall. Each row represents a different fibrillatory wave. The layout and color-coding are the same as in Figure 2. 


\section{DISCUSSION}

This study demonstrates that during pacing atrial repolarization displays rather synchronous behavior, resulting in local prolongation of action potential duration at the site of stimulation. By contrast, during AF atrial repolarization shows a wave-like propagating behavior with a pattern that is similar to the preceding activation. Overall, there was a higher degree of linear correlation between the activation and repolarization times during AF than during atrial pacing.

\section{Atrial repolarization and electrotonic interactions}

Both the spread of depolarization and repolarization depend on electrotonic interactions. Whereas active propagation spreads with a certain velocity, typically $0.5-1 \mathrm{~m} / \mathrm{s}$, during the action potential upstroke in the working myocardium, the spread of passive potentials is instantaneous. The distance over which potentials spread passively is determined by the length constant $l=\sqrt{ }\left(R_{m} / R_{i}\right)$, where $R_{i}$ is internal resistance and $R_{m}$ is membrane resistance. ${ }^{26}$ The internal resistance $\mathrm{R}_{\mathrm{i}}$ is mainly determined by the gap junctional coupling, which does not vary over the cardiac cycle. However, the membrane resistance $\mathrm{R}_{\mathrm{m}}$ does strongly depend on the phase of the action potential. During the action potential upstroke the membrane resistance is relatively low due to the massive activation of sodium channels. In comparison, the membrane resistance increases strongly during repolarization phase.

Despite substantially different activation patterns, obtained as a result of stimulation at different pacing sites, the observed repolarization patterns were relatively similar to one another. The differences in membrane resistance during the time course of action potential provide an explanation why local repolarization patterns are not affected by the site of the earliest activation. As a direct consequence of Ohm's law, the increased membrane resistance during repolarization allows small ionic currents to cause relatively large membrane voltage differences. The cardiomyocytes that repolarize early are the first ones that reach the state of highest membrane resistance. In the presence of a repolarization current, they exhibit larger changes in membrane voltage than the cardiomyocytes that repolarize later. Therefore, they act as a source that accelerates repolarization of the cardiomyocytes to which they are electrically coupled to, forcing their membrane voltage into the range of activation of inward rectifier current. ${ }^{14}$ As a consequence of this process, repolarization of cardiac tissue becomes more synchronized. ${ }^{27}$ On the other hand, the diastolic membrane resistance is much lower than the membrane resistance at repolarization phase. ${ }^{14}$ Considerably larger ionic currents are therefore necessary to depolarize the cell membrane. Depolarization thus propagates through the cardiac tissue less synchronously, forming irregular propagating activation wavefronts. 


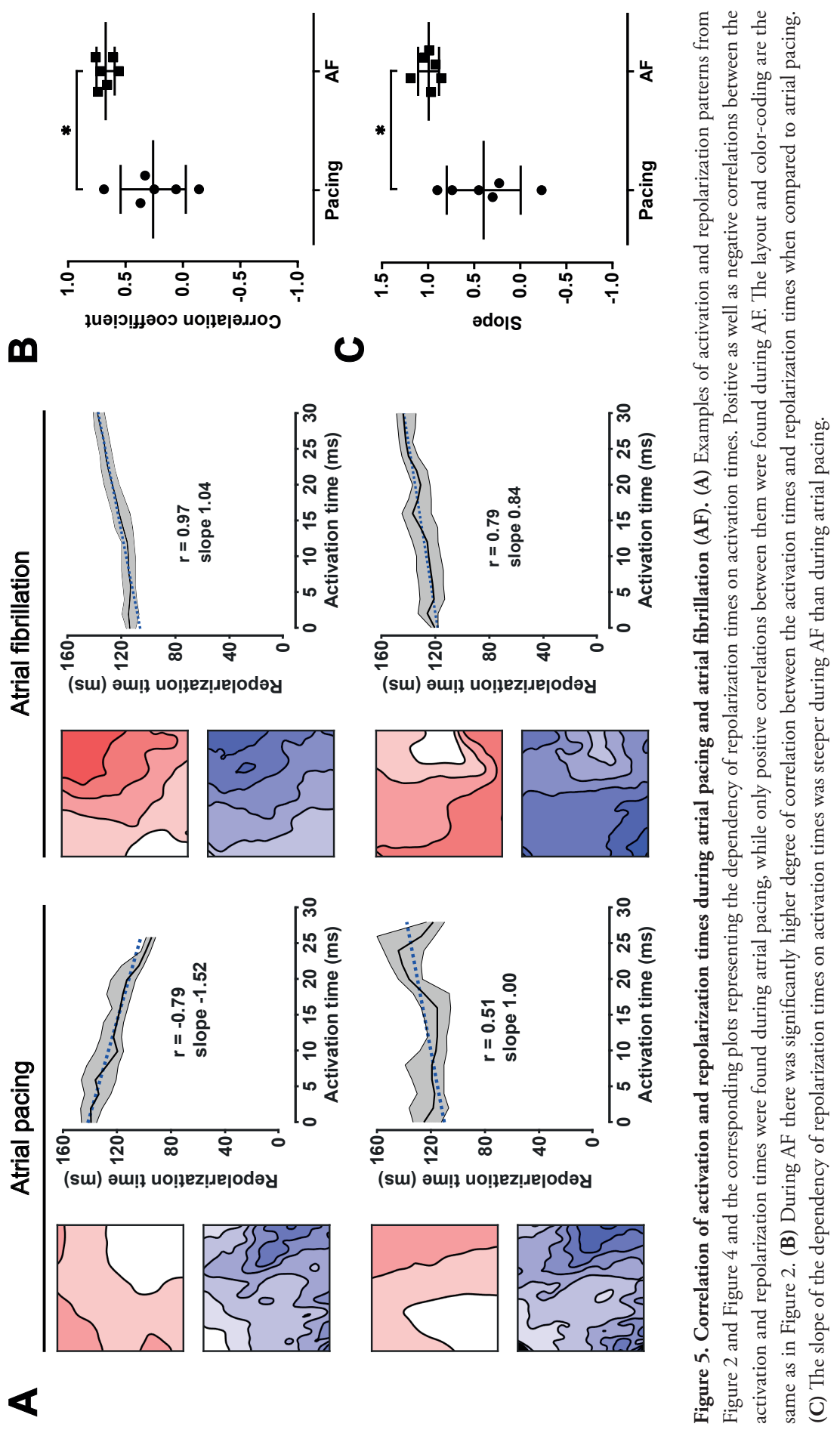


In ventricular cardiomyocytes the difference between the membrane resistance at depolarization and repolarization is pronounced, mainly due to the increased inward rectifier current. ${ }^{28}$ Atrial cardiomyocytes exhibit substantially higher resting membrane resistance than ventricular cardiomyocytes (350 vs. $15 \mathrm{M} \Omega$, respectively). This feature causes that atrial cardiomyocytes can be easily stimulated from sources with low electrical current like sinus node. ${ }^{28}$ Further increase of atrial resting membrane resistance may result in synchronized behavior of depolarization. This is the case in sinoatrial cells that due to their high input resistance (400$500 \mathrm{M} \Omega)^{29}$ exhibit synchronous depolarization. ${ }^{30}$ However, as the resting membrane resistance of atrial cardiomyocytes is lower than the resting membrane resistance of sinoatrial cells, the depolarization in atria still maintains the character of a propagating wave.

\section{Local prolongation of action potential duration at the site of pacing}

The differences in local activation and repolarization patterns during atrial pacing result in local prolongation of $\mathrm{APD}$ at the stimulation site. The $\mathrm{APD}_{80}$ prolongation at the site of pacing $(8 \pm 5$ $\mathrm{ms}, 6.6 \pm 4.2 \%$ ) may have consequences for measurements of atrial effective refractory period (ERP). In the human atrium, a strong linear relationship has been shown between myocardial repolarization and refractoriness, represented by APD and ERP, respectively. ${ }^{31}$ As APD at a particular site is longer during pacing than the intrinsic APD at this site, our findings indicate that also ERP acquired during pacing at this site might be longer than the 'true'refractory period, i.e., the shortest intrinsic time between two subsequent activations. From this perspective, ERP measured by a pacing protocol might have a tendency to systematically overestimate the actual atrial refractoriness.

To confirm our experimental findings regarding $\mathrm{APD}_{80}$ prolongation at the site of pacing, we performed a computer simulation of pacing in a model of atrial tissue monolayer that implements electrophysiological behavior of human atrial cells. ${ }^{25}$ The result of the simulation clearly showed the phenomenon of local APD prolongation at the stimulation site. Implementing the effect of tissue anisotropy by defining different electrical conductivities in the horizontal and vertical direction, the resulting activation pattern and the pattern of APD distribution resembled the respective patterns acquired from our optical mapping experiments (see the activation pattern and $\mathrm{APD}_{80}$ distribution from the Figure 2, top row and the Figure 3 for comparison). A similar observation about local prolongation of APD at the site of pacing has been also described by a computational study in homogenous cardiac tissue. ${ }^{32}$

Several studies on the ventricular myocardium have reported prolonged APD at the site of pacing as well. Negative linear relationship between the activation time and APD during pacing has been described in ventricular endocardium of healthy humans. ${ }^{33}$ Optical mapping studies in perfused ventricles of rabbits ${ }^{34}$ and rats $^{35}$ also demonstrated negative relationship between 
the activation time and APD. Since the duration of ventricular APD can be easily derived from activation-recovery intervals, ${ }^{36}$ it is possible to investigate local APD and repolarization patterns in situ. ${ }^{37}$ However, obtaining this information from atria still remains technically challenging. ${ }^{15}$

\section{The similarity of activation and repolarization patterns is a cause of 'linking' of fibrillatory waves}

The strong correlation between activation and repolarization times during AF shown in our study may in part explain the phenomenon of 'linking' of fibrillatory waves. This phenomenon describes the general tendency of excitation wavefronts to follow the paths of previous excitation. During AF the action potentials are generally shortened, and the activation patterns largely determine the spatial distribution of refractoriness. The atrial tissue thus becomes reexcitable in a similar sequence as the last excitation occurred. When the atrial activation is premature, the spatial distribution of refractoriness substantially affects the next excitation wave, ${ }^{27}$ and this wave tends to follow the same path as the preceding excitation wave. The two waves exhibit similar direction of propagation and therefore appear to be 'linked'. Evidence for this phenomenon has been provided in human atria ${ }^{38,39}$ but it has been also described in goats. ${ }^{40}$ Visual inspection of our optical mapping recordings of AF also confirmed the presence of linking of fibrillatory waves (data not shown).

\section{Novel method for assessment of atrial repolarization during AF}

The current study presents a novel method for assessment of atrial repolarization during AF. The method further develops the algorithm for detection of atrial activations from optical mapping data described in chapter 5. The main advantage of the implemented approach is beat-to-beat analysis of activation and repolarization times, allowing to describe the spatial distribution of these parameters. Although several studies used optical mapping recordings from perfused heart preparations to investigate the mechanisms underlying AF, their data processing methods were predominantly focused on reconstruction of activation patterns ${ }^{17,41,42}$ or analysis in frequency ${ }^{41,43}$ or phase domain. ${ }^{17,44,45}$ In this context, our study is the first one that attempts to provide the description of the relationship between activation and repolarization patterns during AF on a beat-to beat level.

In general, assessment of atrial repolarization, especially during AF, is technically challenging. There have been several studies that attempted to assess atrial repolarization. Narayan et al. recorded local repolarization times in patients during AF using monophasic action potential (MAP) catheters. However, their analysis of MAP duration was manual and thus very time consuming. ${ }^{46,47}$ It has been demonstrated in pigs that atrial repolarization can be determined from standard clinical catheters during regular rhythms. ${ }^{15}$ Nevertheless, these results still require further validation. ${ }^{16}$ Although the catheter-based methods allowed to determine atrial 
repolarization locally and even during $\mathrm{AF}$, reconstruction of repolarization patterns is still beyond their limits. From that perspective, optical mapping is still an irreplaceable method to study these phenomena.

\section{LIMITATIONS}

The determination of repolarization times during AF, as applied in this study, has its considerable limitations. The definition of the repolarization level as the function that interpolates local minima of a pixel signal provided us with a solution how to determine repolarization times independently from the subsequent activations. However, the electrophysiological full repolarization level is usually not reached during AF and thus cannot be determined. As a consequence of that, the reported values of $\mathrm{APD}_{80}$ obtained during AF might be systematically underestimated. Nevertheless, visual inspection of the algorithm performance has shown that the relationship between activation and repolarization patterns should not be influenced by this aspect. The calculation of $\mathrm{APD}_{80}$ from the recordings of atrial pacing has not been affected by this aspect either, since the action potentials reached full level of repolarization.

Using the goat hearts in which AF was induced and maintained for 3 weeks allowed us to investigate persistent AF with electrical remodeling only. Due to that, the analyzed fibrillatory patterns are relatively simple. If a later time point was used, the atrial remodeling would be accompanied with structural changes in atria, resulting in more complex activation patterns and smaller fibrillatory waves. ${ }^{48}$ It would be of interest to investigate how the interaction between activation and repolarization patterns changes when activation patterns become more complex due to structural remodeling of atria.

We used the excitation-contraction uncoupler blebbistatin in perfused heart preparations. It has been shown that in perfused rabbit hearts blebbistatin significantly prolongs ventricular APD, affects APD restitution and reduces complexity and inducibility of ventricular fibrillation. ${ }^{49,50}$ However, there is also an evidence showing no effects of blebbistatin on electrophysiological properties. ${ }^{51}$ It is therefore conceivable that the effect of blebbistatin is preparation- and species-dependent. ${ }^{50}$ Since there is no study on the effect of blebbistatin in perfused goat hearts, we cannot exclude the possibility that the perfused goat heart preparations uncoupled with blebbistatin exhibit APD prolongation and reduced AF complexity. Nevertheless, none of the aforementioned studies indicated that blebbistatin affects the nature of electrotonic interactions. Considering also the fact that our experimental results have been confirmed by computer simulation, we assume that blebbistatin had only a limited effect on the studied phenomena. 
The applied computational model of human atrial tissue monolayer was relatively simple. In the results of the computer simulation we observed accelerated shortening of APD towards the model boundaries, which has been shown as a common feature of cardiac tissue models with simple geometries. ${ }^{32}$ However, as the results of the simulation clearly showed APD prolongation at the site of pacing and thus confirmed our experimental observations, we found the use of more sophisticated computer models of human atria unnecessary.

\section{CONCLUSION}

During atrial pacing, the action potential duration at the site of stimulation prolongs, leading to rather synchronous repolarization throughout the field of view. By contrast, the repolarization during AF shows a wave-like propagating behavior, with a pattern that is similar to the preceding activation. 


\section{REFERENCES}

1. Durrer D, van Dam RT, Freud GE, Janse MJ, Meijler FL, Arzbaecher RC. Total excitation of the Isolated Human Heart. Circulation 1970;41:899-912.

2. Josephson ME, Scharf DL, Kastor JA, Kitchen JG. Atrial endocardial activation in man: electrode catheter technique for endocardial mapping. Am J Cardiol 1977;39:972-981.

3. Ramanathan C, Jia P, Ghanem R, Ryu K, Rudy Y. Activation and repolarization of the normal human heart under complete physiological conditions. Proc Natl Acad Sci U S A 2006;103:63096314.

4. Opthof T, Remme CA, Jorge E, Noriega F, Wiegerinck RF, Tasiam A, et al. Cardiac activationrepolarizaiton patterns and ion channel expression mapping in intact isolated normal human hearts. Heart Rhythm 2017;14:265-272.

5. Coronel R, Wilms-Schopman FJG, Opthof T, Janse MJ. Dispersion of repolarization and arrhythmogenesis. Heart Rhythm 2009;6:537-543.

6. Li Z, Hertervig E, Yuan S, Yang Y, Lin Z, Olsson SB. Dispersion of atrial repolarization in patients with paroxysmal atrial fibrillation. Europace 2001;3:285-291.

7. Hertervig E, Li Z, Kongstad O, Holm M, Olsson SB, Yuan S. Global dispersion of right atrial repolarization in healthy pigs and patients. Scand Cardiovasc J 2003;37:329-333.

8. Ihara Z, van Oosterom A, Hoekema R. Atrial repolarization as observable during PQ interval. J Electrocardiol 2006;39:290-297.

9. Tang WH, Ho WH, Chen YJ. Retrieving hidden atrial repolarization waves from standard surface ECGs. Biomed Eng Online 2018;17:146.

10. Krueger MW, Dorn A, Keller DUJ, Holmqvist F, Carlson J, Platonov PG, et al. In-silico modeling of atrial repolarization in normal and atrial fibrillation remodeled state. Med Biol Eng Comput 2013;51:1105-1119.

11. Holmqvist F, Carlson J, Platonov PG. Detailed ECG analysis of atrial repolarization in humans. Ann Noninvasive Electrocardiol 2009;14:13-18.

12. Narayan SM, Franz MR, Clopton P, Pruvot EJ, Krummen DE. Repolarization alternans reveals vulnerability to human atrial fibrillation. Circulation 2011;123:2922-2930.

13. Spitzer KW, Sato N, Tanaka H, Firek L, Zaniboni M, Giles WR. Electrotonic modulation of electrical activity in rabbit atrioventricular node myocytes. Am J Physiol 1997;273:H767-H776.

14. Zaniboni M, Pollard AE, Yang L, Spitzer KW. Beat-to-beat repolarization variability in ventricular myocytes and its suppresion by electrical coupling. Am J Physiol Heart Circ Physiol 2000;278:H677-H687.

15. Verrier RL, Fuller H, Justo F, Nearing BD, Rajamani S, Belardinelli L. Unmasking atrial repolarization to assess alternans, spatiotemporal heterogeneity, and susceptibility to atrial fibrillation. Heart Rhythm 2016;13:953-961.

16. Narayan SM, Zaman JA, Baykaner T, Franz MR. Atrial fibrillation: Can electrograms be interpreted without repolarization information? Heart Rhythm 2016;13:962-963. 
17. Laughner JI, Ng FS, Sulkin MS, Arthur RM, Efimov IR. Processing and analysis of cardiac optical mapping data obtained with potentiometric dyes. Am J Physiol Heart Circ Physiol 2012;303:H753765.

18. Nygren A, Lomax AE, Giles WR. Heterogeneity of action potential durations in isolated mouse left and right atria recorded using voltage-sensitive dye mapping. Am J Physiol Heart Circ Physiol 2004;287:H2634-H2643.

19. Verheule S, Wilson E, Banthia S, Everett TH, Shanbhag S, Sih HJ, et al. Direction-dependent conduction abnormalities in a canine model of atrial fibrillation due to chronic atrial dilatation. Am J Physiol Heart Circ Physiol 2004;287:H634-H644.

20. Li N, Csepe TA, Hansen BJ, Sul LV, Kalyanasundaram A, Zakharkin S, et al. Adenosine-induced atrial fibrillation: localized reentrant drivers in lateral right atria due to heterogeneous expression of adenosine A1 receptors and GIRK4 subunits in the human heart. Circulation 2016;134:486-498.

21. Van Wagoner DR. Electrophysiological remodeling in human atrial fibrillation. Pacing Clin Electrophysiol 2003;26:1572-1575.

22. Wijffels MCEF, Kirchhof CJHJ, Dorland R, Allessie MA. Atrial Fibrillation Begets Atrial Fibrillation: A Study in Awake Chronically Instrumented Goats. Circulation 1995;92:1954-1968.

23. Kuijpers NH, Keldermann RH, Arts T, Hilbers PA. Computer simulations of successful defibrillation in decoupled and non-uniform cardiac tissue. Europace 2005;7 Suppl 2:166-177.

24. Verheule S, Tuyls E, Gharaviri A, Hulsmans S, van Hunnik A, Kuiper M, et al. Loss of continuity in the thin epicardial layer because of endomysial fibrosis increases the complexity of atrial fibrillatory conduction. Circ Arrhythm Electrophysiol 2013;6:202-211.

25. Courtemanche M, Ramirez RJ, Nattel S. Ionic mechanisms underlying human atrial action potential properties: insights from a mathematical model. Am J Physiol 1998;275:H301-321.

26. Beierlein M. Chapter 17 - Cable Properties and Information Processing in Dendrites. In: Byrne JH, Heidelberger R, Waxham MN, editors. From Molecules to Networks (Third Edition). Boston: Academic Press; 2014. p. 509-529.

27. Spach MS, Dolber PC, Heidlage JF. Interaction of inhomogeneities of repolarization with anisotropic propagation in dog atria. A mechanism for both preventing and initiating reentry. Circ Res 1989;65:1612-1631.

28. Golod DA, Kumar R, Joyner RW. Determinants of action potential initiation in isolated rabbit atrial and ventricular myocytes. Am J Physiol 1998;274:H1902-H1913.

29. Joyner RW, Kumar R, Golod DA, Wilders R, Jongsma HJ, Verheijck EE, et al. Electrical interactions between a rabbit atrial cell and a nodal cell model. Am J Physiol 1998;274:H2152-2162.

30. Jalife J. Mutual entrainment and electrical coupling as mechanisms for synchronous firing of rabbit sino-atrial pace-maker cells. J Physiol 1984;356:221-243.

31. Bode F, Kilborn M, Karasik P, Franz MR. The repolarization-excitability relationship in the human righ atrium is unaffected by cycle length, recording site and prior arrhythmias. J Am Coll Cardiol 2001;37:920-925.

32. Cherry EM, Fenton FH. Effects of boundaries and geometry on the spatial distribution of action potential duration in cardiac tissue. J Theor Biol 2011;285:164-176. 
33. Franz MR, Bargheer K, Rafflenbeul W, Haverich A, Lichtlen PR. Monophasic action potential mapping in human subjects with normal electrocardiograms: direct evidence for the genesis of the T wave. Circulation 1987;75:379-386.

34. Myles RC, Bernus O, Burton FL, Cobbe SM, Smith GL. Effect of activation sequence on transmural patterns of repolarization and action potential duraiton in rabbit ventricular myocardium. Am J Physiol Heart Circ Physiol 2010;299:H1812-H1822.

35. Walton RD, Benson AP, Hardy MEL, White E, Bernus O. Electrophysiological and structural determinants of electrotonic modulation of repolarization by the activation sequence. Front Physiol 2013;4:281.

36. Wyatt RF, Burgess MJ, Evans AK, Lux RL, Abildskov JA, Tsutsumi T. Estimation of ventricular transmembrane action potential durations and repolarization times from unipolar electrograms. Am J Cardiol 1981;42:488.

37. Chen PS, Moser KM, Dembitsky WP, Auger WR, Daily PO, Calisi CM, et al. Epicardial activation and repolarization patterns in patients with right ventricular hypertrophy. Circulation 1991;83:104118.

38. Gerstenfeld EP, Sahakian AV, Swiryn S. Evidence for transient linking of atrial excitation during atrial fibrillation. Circulation 1992;86:375-382.

39. Schoenwald AT, Sahakian AV, Sih HJ, Swiryn S. Further observations of "linking" of atrial excitation during clinical atrial fibrillation. Pacing Clin Electrophysiol 1998;21:25-34.

40. Shan Z, van der Voort PH, Blaauw Y, Duytschaever M, Allessie MA. Fractionation of electrograms and linking of activation during pharmacologic cardioversion of persistent atrial fibrillation in the goat. J Cardiovasc Electrophysiol 2004;15:572-580.

41. Skanes AC, Mandapati R, Berenfeld O, Davidenko JM, Jalife J. Spatiotemporal periodicity during atrial fibrillation in the isolated sheep heart. Circulation 1998;98:1236-1248.

42. Berenfeld O, Zaitsev AV, Mironov SF, Pertsov AM, Jalife J. Frequency-dependent breakdown of wave propagation into fibrillatory conduction across the pectinate muscle network in the isolated sheep right atrium. Circ Res 2002;90:1173-1180.

43. Zaitsev AV, Berenfeld O, Mironov SF, Jalife J, Pertsov AM. Distribution of excitation frequencies on the epicardial and endocardial surfaces of fibrillating ventricular wall of the sheep heart. Circ Res 2000;86:408-417.

44. Chen J, Mandapati R, Berenfeld O, Skanes AC, Gray RA, Jalife J. Dynamics of wavelets and their role in atrial fibrillation in the isolated sheep heart. Cardiovasc Res 2000;48:220-232.

45. Filgueiras-Rama D, Price NF, Martins RP, Yamazaki M, Avula UM, Kaur K, et al. Long-term frequency gradients during persistent atrial fibrillation in sheep are associated with stable sources in the left atrium. Circ Arrhythm Electrophysiol 2012;5:1160-1167.

46. Narayan SM, Franz MR. Quantifying fractionation and rate in human atrial fibrillation using monophasic action potentials: implications for substrate mapping. Europace 2007;9 Suppl 6:vi8995.

47. Narayan SM, Krummen DE, Kahn AM, Karasik PL, Franz MR. Evaluating fluctuations in human atrial fibrillatory cycle length using monophasic action potentials. Pacing Clin Electrophysiol 2006;29:1209-1218. 
48. Verheule S, Tuyls E, van Hunnik A, Kuiper M, Schotten U, Allessie M. Fibrillatory conduction in the atrial free walls of goats in persistent and permanent atrial fibrillation. Circ Arrhythm Electrophysiol 2010;3:590-599.

49. Brack KE, Narang R, Winter J, Ng A. The mechanical uncoupler blebbistatin is associated with significant electrophysiological effects in the isolated rabbit heart. Exp Physiol 2013;98:1009-1027.

50. Kappadan V, Telele S, Uzelac I, Fenton FH, Parlitz U, Luther S, et al. High-resolution optical measurement of cardiac restitution, contraction, and fibrillation dynamics in beating vs. blebbistatin-uncoupled isolated rabbit hearts. Front Physiol 2020;11:464.

51. Fedorov VV, Lozinsky IT, Sosunov EA, Anyukhovsky EP, Rosen MR, Balke CW, et al. Application of blebbistatin as an excitation-contraction uncoupler for electrophysiologic study of rat and rabbit hearts. Heart Rhythm 2007;4:619-626. 


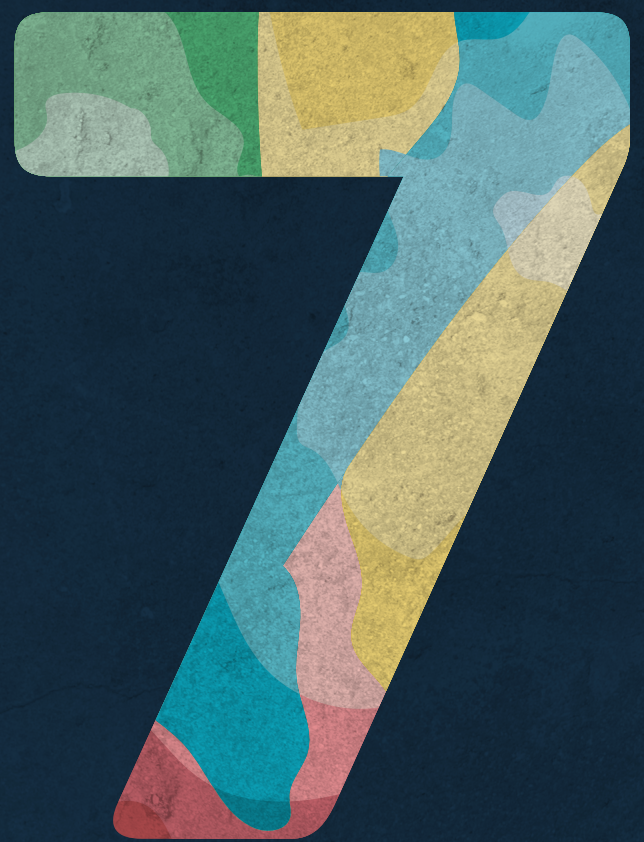


General discussion 

Atrial fibrillation (AF) represents a major challenge for public health and a significant socioeconomic burden. It is expected that in the next 20 years the number of AF patients in the EU countries will increase by $50 \%$, leading to up to 17 million patients with this arrhythmia by $2050 .^{1}$

Prevention of stroke by anticoagulation therapy is a cornerstone of clinical management of AF. ${ }^{2}$ In addition, management of the rapid ventricular rate that accompanies $\mathrm{AF}$ is necessary, since it is responsible for most of the AF-related symptoms. ${ }^{3}$ The current guidelines for diagnosis and management of AF recommend two main strategies: restoration of sinus rhythm (rhythm control) or limitation of ventricular rate (rate control). ${ }^{2}$ Rhythm control intuitively appears to be a preferred therapy, since restoration of sinus rhythm allows normal physiological function of the heart, including normal regulation of heart rate. Restoration of sinus rhythm can be achieved either by antiarrhythmic drugs (AADs), electrical cardioversion or by catheter ablation. ${ }^{2,4}$ By contrast, rate control can be seen as a treatment of symptoms, aiming to reduce the ventricular rate in the presence of AF. The common therapeutic strategy for rate control is administration of agents that block atrioventricular nodal conduction. When insufficient, rate control can be achieved by ablation of the atrioventricular junction and implantation of a pacemaker. $^{3}$

With respect to rhythm control as an approach that restores physiological function of the heart, it may be surprising that large clinical trials did not show its superiority over rate control. ${ }^{3,5-8}$ The comparable outcome of both therapeutic strategies can be attributed to two reasons. First, the benefits of rhythm control therapy with AADs might be outweighed by the side effects that are associated with the use of these drugs. These include proarrhythmic effects in ventricles and an increased risk of cardiovascular events and deaths. ${ }^{9,10}$ The second reason might be late initiation of the antiarrhythmic treatment, when AF is already persistent and the atria structurally remodeled. In these conditions the efficacy of AADs for treatment of AF substantially decreases. ${ }^{11}$

The recently published Early treatment of Atrial fibrillation for Stroke prevention Trial (EAST) investigated the potential benefits of early initiation of rhythm control therapy in patients with recently diagnosed AF. ${ }^{12}$ The trial demonstrated that compared to usual care, the strategy of early rhythm control is associated with better outcomes (Figure 1). Interestingly, such result has been achieved despite higher occurrence of serious adverse events in the rhythm control group ( $4.9 \%$ vs. $1.4 \%$, early rhythm control vs. usual care, respectively). Moreover, only $84.9 \%$ of patients in the rhythm control group were in sinus rhythm after 1 year of follow up, ${ }^{12}$ indicating that the rhythm control intervention still failed to maintain sinus rhythm in substantial number of AF patients. It is therefore conceivable that even better outcomes could have been achieved if the rhythm control AADs were more efficient in restoration of sinus 
rhythm and fewer cardiovascular adverse effects were associated with their use. The findings of the EAST trial thus further encourage development of new pharmacological approaches for treatment of AF that would be safe and efficient at the same time.

In this general discussion, we would like to address several issues related to the lack of efficacy and atrial selectivity of currently available AADs for rhythm control therapy:

1. Are there more atrial-selective strategies for treatment of AF? The use of currently available AADs is limited by their side effects, including ventricular proarrhythmic effects. Development of truly atrial-selective AADs is therefore desirable, as exemplified by the selective inhibitors of acetylcholine-activated potassium current $\left(\mathrm{I}_{\mathrm{KACh}}\right)$ and small conductance calcium-activated potassium current $\left(\mathrm{I}_{\mathrm{SK}}\right)$ investigated in the chapter $\mathbf{2}$ and chapter $\mathbf{3}$ of this thesis.

2. How do AADs terminate AF? The knowledge about the mechanisms how AADs actually terminate AF is only limited. In order to identify common mechanisms of AAD-induced AF termination, we analyzed a collection of data sets acquired during studies investigating the antiarrhythmic effects of AADs in the goat model of AF. The study presented in chapter $\mathbf{4}$ describes the changes in atrial electrophysiology during the last seconds that precede cardioversion of AF, providing new insights about the mechanisms leading to the arrhythmia termination.

3. How does structural remodeling affect the antiarrhythmic effects of $A A D$ s? Structural remodeling of atria leads to complex fibrillatory conduction. The antiarrhythmic effects of currently available AADs are insufficient in these conditions, leading to the lack of cardioversion efficacy. Optical mapping of intact atria allows to provide a detailed analysis of complex activation patterns. However, the potential of this method has not been fully exploited due to the lack of existing methods for the analysis of fibrillatory conduction. In chapter $\mathbf{5}$ we have developed a propagation-based algorithm to advance this type of analysis, allowing more detailed evaluation of complex activation patterns. The method has a potential to elucidate the mechanisms underlying the low efficacy of available AADs in structurally remodeled atria, as well as to facilitate the development of antiarrhythmic strategies that would work under such conditions. 


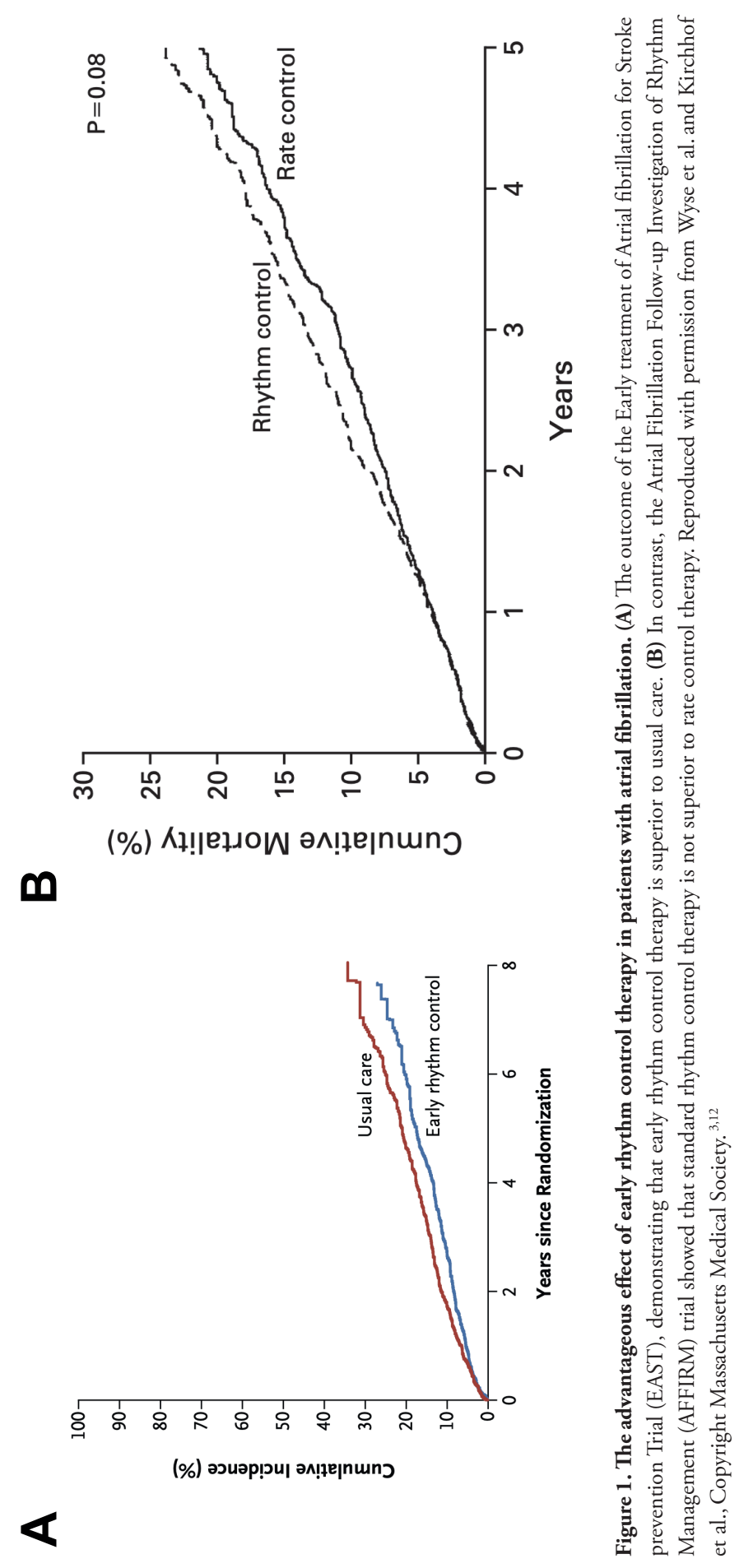


4. Does prolongation of action potential duration (APD) have proarrhythmic effects in atria? The efficacy of currently available AADs to cardiovert AF decreases in time as a result of progressive AF-induced remodeling. This decline in cardioversion efficacy might be, in part, caused by possible atrial proarrhythmic effects the AADs have. The AAD-induced prolongation of APD may inhibit reentry, but at the same time, it can potentially trigger arrhythmias by increasing the likelihood of early afterdepolarizations and causing heterogeneity in APD prolongation. As we are currently lacking the tools that would allow studying these phenomena, in chapter $\mathbf{6}$ we present a method for analysis of repolarization patterns during fibrillatory conduction that can potentially facilitate investigation of these proarrhythmic mechanisms.

\section{NEW ATRIAL-SELECTIVE STRATEGIES FOR PHARMACOLOGICAL TREATMENT OF AF}

The limited cardioversion efficacy of currently available AADs, together with their ventricular proarrhythmic effects and other side effects, creates an urgent need for improvement of the pharmacological therapy for AF treatment. Designing atrial-selective AADs, i.e., drugs with predominant effect in atria, may allow administration of doses that would prolong AF cycle length to the degree that would lead to AF termination without significant side effects. Such drugs can be designed by aiming at atrial-specific therapeutic targets. Several ion channels predominantly expressed in atrial cardiomyocytes have received substantial attention from the research community and were investigated as potential targets for treatment of AF. ${ }^{13}$ At the moment, the most promising candidates are ultra-rapid delayed rectifier current $\left(\mathrm{I}_{\text {Kur }}\right)$, acetylcholine-activated potassium current $\left(\mathrm{I}_{\mathrm{KACh}}\right)$, small conductance calcium-activated potassium current $\left(\mathrm{I}_{\mathrm{SK}}\right)$ and two-pore domain channels $\left(\mathrm{I}_{\mathrm{K} 2 \mathrm{p}}\right) \cdot{ }^{14}$ Another alternative for an atrial-selective AF therapy could be exploitation of the differences in biophysical properties of sodium currents between the atria and ventricles. ${ }^{15}$ It has been proposed that use-dependent sodium channel block, as provided e.g., by antianginal agent ranolazine, ${ }^{16}$ could potentially represent an interesting atrial-selective strategy for treatment of AF.

Some of clinically used AADs, including amiodarone, ${ }^{17}$ dronedarone ${ }^{18}$ or vernakalant, ${ }^{19}$ inhibit multiple ion channels, which might be a feature that increases their efficacy to terminate AF. However, these effects are often not atrial-specific, thereby enhancing the risk of side effects, and the effects on various ion currents cannot be tailored to the stage of AF in the individual patient. Therefore, combined treatment with several specific, atrial-selective ion channel blockers could represent an interesting atrial-selective strategy, using synergistic effects of AADs in cardioverting AF while keeping the doses below the range that would produce (ventricular) side effects. Several studies demonstrated the potential of such a combination therapy in animal model ${ }^{20,21}$ and in silico. ${ }^{22,23}$ 
In this thesis, we have studied the antiarrhythmic effects of $\mathrm{I}_{\mathrm{KACh}}$ (chapter 2) and $\mathrm{I}_{\mathrm{SK}}$ inhibition (chapter 3). Using a goat model of pacing-induced AF, we investigated antiarrhythmic properties and cardioversion efficacy of newly developed compounds XAF-1407 and AP14145 that inhibit $\mathrm{I}_{\mathrm{KACh}}$ and $\mathrm{I}_{\mathrm{SK}}$, respectively.

\section{INHIBITION OF I KACh $_{\text {AND I }}$ IN ANIMAL MODELS OF AF}

$\mathrm{I}_{\mathrm{KACh}}$ is an inwardly rectifying current that contributes to phase 3 repolarization and stabilization of the resting membrane potential. It is predominantly expressed in atria, ${ }^{24}$ sinoatrial node ${ }^{25}$ and atrioventricular node. ${ }^{26,27} \mathrm{I}_{\mathrm{KACh}}$ channels are formed as heterotetramers composed of two $\mathrm{K}_{\mathrm{ir}} 3.1$ and two $\mathrm{K}_{\mathrm{ir}} 3.4$ subunits. ${ }^{28}$ Under physiological conditions, $\mathrm{I}_{\mathrm{KACh}}$ channels are activated by vagal stimulation. However, constitutively active $\mathrm{I}_{\mathrm{KACh}}$ has been found in patients with chronic AF, increasing the total inward rectifier potassium current in AF-remodeled atria. ${ }^{29} \mathrm{I}_{\mathrm{KACh}}$ received considerable attention as a potential therapeutic target for treatment of AF mainly because its pharmacological inhibition may represent an atrial-selective as well as AF-selective therapy.

SK channels have three different subtypes, of which two are dominantly present in the atria. ${ }^{30-32}$ SK channels open when the concentration of intracellular calcium is sufficiently high, ${ }^{33}$ leading to a potassium current during the action potential plateau and repolarization that shortens the atrial APD. ${ }^{30}$ SK channels participate in depolarization of the resting membrane potential and affect the steepness of the action potential upstroke. ${ }^{30,34}$ Therefore, it is conceivable that SK inhibition may result in both class III effects (prolongation of action potential duration) as well as in class I effect (deceleration of action potential upstroke).

Studying the antiarrhythmic effects of $\mathrm{I}_{\mathrm{KACh}}$ inhibitor XAF-140735 and of $\mathrm{I}_{\mathrm{SK}}$ inhibitor AP14145 $5^{36}$ in the goat model of pacing-induced AF, we have shown that both compounds substantially prolong atrial effective refractory period (aERP). Our study on $\mathrm{I}_{\mathrm{SK}}$ inhibition with AP14145 confirms previous experimental findings with this antiarrhythmic agent ${ }^{36,37}$ and is also in agreement with the experimental studies with SK channel inhibitor NS8593, showing a substantial effect of $\mathrm{I}_{\mathrm{SK}}$ inhibition on aERP prolongation. ${ }^{38,39}$ Similarly, the degree of aERP prolongation after the administration of XAF-1407 in goats was comparable to the results reported in horses ${ }^{35}$ and in other studies with $\mathrm{I}_{\mathrm{KACh}}$ inhibitors. ${ }^{40-42}$ We also demonstrated that $\mathrm{I}_{\mathrm{KACh}}$ inhibition with XAF-1407 had stronger effect in electrically remodeled atria than in normal atria. The findings presented in chapter 2 agree with the hypothesis that constitutively active $I_{\text {KACh }}$ contributes to the shortening of aERP in AF-remodeled atria. ${ }^{29,43}$ Inhibition of $\mathrm{I}_{\mathrm{KACh}}$ may therefore represent not only atrial-selective, but also AF-selective strategy for pharmacological treatment of AF. 
The administration of XAF-1407 resulted in termination of persistent AF in 8 out of 9 goats $(89 \%)$. At a comparable time point, cardioversion rate of $70 \%$ has been reported with the same dose of XAF-1407 in horses. ${ }^{35}$ The cardioversion rates with other $\mathrm{I}_{\mathrm{KACh}}$ inhibiting agents in dogs with AF ranged from $62.5 \%$ to $100 \%,{ }^{41,42}$ indicating that selective $\mathrm{I}_{\mathrm{KACh}}$ inhibitors may represent a promising strategy for pharmacological treatment of AF. Similarly, the administration of AP14145 resulted in termination of persistent AF in 5 out of 6 goats (83\%), indicating high efficacy of the compound to cardiovert AF. The efficacy of AP14145 to terminate $\mathrm{AF}$ was also evaluated in comparison to vernakalant, the most recent $\mathrm{AAD}$ approved for European market, ${ }^{44-48}$ indicating that AP14145 is able to terminate persistent AF that vernakalant fails to cardiovert. ${ }^{37}$ Showing that vernakalant cardioverted 5 out of 9 goats $(56 \%)$, we demonstrated high efficacy of XAF-1407 to terminate AF as well. Being more effective in cardioverting AF than vernakalant, the inhibition of $\mathrm{I}_{\mathrm{SK}}$ with $\mathrm{AP} 14145$ as well as the inhibition of $\mathrm{I}_{\mathrm{KACh}}$ with XAF-1407 appear to be potent strategies for AF termination.

A rate-dependent reduction of atrial conduction velocity was observed in goats when either AP14145 or XAF-1407 was administered, indicating a class I effect of these antiarrhythmic agents. As the goat model of pacing-induced AF did not allow us to study the selectivity of AP14145 and XAF-1407 to inhibit different ion currents in goat atria, it was not possible to investigate the mechanism underlying the functional class I effect of these drugs. Additional patch clamp experiments would be necessary to elucidate the class I effect of AP14145 and XAF-1407. We speculate that the observed deceleration of atrial conduction can be explained by depolarization of the resting membrane potential. No class I effect has been reported previously for selective $I_{\text {KACh }}$ inhibitors NTC-801, AZD2927 and A7071.40,42 However, decrease of both action potential upstroke and amplitude have been reported for SK channel inhibitors NS8593 and ICAGEN. ${ }^{30}$ The possible mechanism of the functional class I effect is that $\mathrm{I}_{\mathrm{SK}}$ contributes to the resting membrane potential and its inhibition depolarizes the resting membrane potential and thereby reduces the availability of sodium channels. ${ }^{30}$ As the synergistic effect of class I and class III has been demonstrated to result in increased antiarrhythmic effect, ${ }^{22}$ this feature might contribute to the high cardioverting efficacies of AP14145 and XAF-1407.

The inhibition of $I_{S K}$ with AP14145 as well as the inhibition of $I_{\text {KACh }}$ with XAF-1407 had only limited effects on ventricular electrophysiology and pump function of goat hearts. The administration of these compounds was not associated with any ventricular proarrhythmic events. The effects of XAF-1407 on ventricular electrophysiology were limited and no ventricular effects were observed for AP14145. Similarly, both compounds had little or no effect on left ventricular pump function. Our work thus confirms previous studies demonstrating that both $\mathrm{I}_{\mathrm{KACh}}$ and $\mathrm{I}_{\mathrm{SK}}$ inhibition have a potential to become safe, atrial-selective strategies for treatment of $\mathrm{AF}^{40,42,49,50}$ Given the relatively high incidence of ventricular proarrhythmic events during 
rhythm control management with currently available AADs, the ventricular safety of $\mathrm{I}_{\mathrm{KACh}}$ and $I_{S K}$ inhibition is a prerequisite for further development of selective inhibitors of these ion currents.

Studying the effects of $\mathrm{I}_{\mathrm{KACh}}$ and $\mathrm{I}_{\mathrm{SK}}$ inhibition in the presence of an arrhythmogenic substrate characterized by electrical remodeling allowed us to investigate the antiarrhythmic effects of XAF-1407 and AP14145 in conditions when AF complexity is low and the arrhythmia is relatively easy to cardiovert. ${ }^{51-53}$ However, in clinical setting AF frequently occurs in patients with underlying heart disease and concomitant structural changes in atria, including increased atrial fibrosis ${ }^{54,55}$ and atrial dilatation. ${ }^{56}$ These structural changes lead to complex fibrillatory conduction and increased stability of AF. ${ }^{11,57,58}$ Therefore, the logical next step for investigating the antiarrhythmic potential of $\mathrm{I}_{\mathrm{KACh}}$ and $\mathrm{I}_{\mathrm{SK}}$ inhibition would be to study the antiarrhythmic effects of these selective inhibitors in animal models with structurally remodeled atria. The models with prolonged AF maintenance ${ }^{11}$ or underlying heart disease ${ }^{59}$ could address the question whether these novel antiarrhythmic targets are efficient to cardiovert complex and therefore more stable AF.

\section{Clinical potential of $\mathrm{I}_{\mathrm{KACh}}$ inhibition for treatment of AF}

There is currently no selective $\mathrm{I}_{\mathrm{KACh}}$ inhibiting drug clinically available for treatment of AF. However, several clinically used AADs exhibit $I_{K A C h}$ inhibiting properties, including dronedarone, ${ }^{60,61}$ amiodarone, ${ }^{62}$ propafenone, ${ }^{63}$ flecainide, ${ }^{63}$ quinidine ${ }^{64,65}$ and vernakalant. ${ }^{66}$ Although it is uncertain to which degree the inhibition of $I_{K A C h}$ contributes to the clinical effectiveness of these drugs, it is likely that the synergistic effect of multiple ion channel inhibition, including $\mathrm{I}_{\mathrm{KACh}}$, contributes to their efficacy to terminate AF.

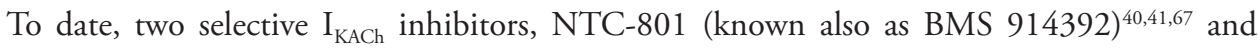
AZD2927 $7^{42}$ have been evaluated in phase 2 clinical trials. However, both drugs failed to meet the primary endpoint and their clinical testing has been discontinued. ${ }^{43}$ The reason why these $\mathrm{I}_{\mathrm{KACh}}$ inhibitors did not show sufficient efficacy in clinical trials remains unclear. An aspect that may play an important role in successful effect of $\mathrm{I}_{\mathrm{KACh}}$ inhibition in humans is the potency of the compound to inhibit the $\mathrm{K}_{\mathrm{ir}} 3.1$ and $\mathrm{K}_{\mathrm{ir}} 3.4$ subunits forming the $\mathrm{I}_{\mathrm{KACh}}$ channels. It has been demonstrated that XAF-1407 and its predecessor XEN-R0706 inhibit both $\mathrm{K}_{\mathrm{ir}} 3.1 / \mathrm{K}_{\mathrm{ir}} 3.4$ heterotetramers and $\mathrm{K}_{\mathrm{ir}} 3.4 / \mathrm{K}_{\mathrm{ir}} 3.4$ homomers with a similar potency. ${ }^{35,43}$ XEN-R0706 was the first antiarrhythmic agent that showed prolongation of $\mathrm{APD}_{90}$ and $\mathrm{aERP}$ ex vivo in atrial tissue from patients with AF but not in the patients in sinus rhythm. ${ }^{43}$ In the same experiments, a selective $\mathrm{I}_{\mathrm{KACh}}$ inhibitor that was inhibiting only $\mathrm{K}_{\mathrm{ir}} 3.1 / \mathrm{K}_{\mathrm{ir}} 3.4$ heterotetramers but not the $\mathrm{K}_{\mathrm{ir}} 3.4 / \mathrm{K}_{\mathrm{ir}} 3.4$ homomers failed to provide the same result, ${ }^{43}$ suggesting the importance of $\mathrm{K}_{\mathrm{ir}} 3.4$ in the AF-remodeled atria of humans. As XAF-1407 inhibits both $\mathrm{K}_{\mathrm{ir}} 3.1 / \mathrm{K}_{\mathrm{ir}} 3.4$ heterotetramers and $\mathrm{K}_{\mathrm{ir}} 3.4 / \mathrm{K}_{\mathrm{ir}} 3.4$ homomers with similar potency, ${ }^{35}$ it might have similar effect 
in AF-remodeled human tissue as its predecessor XEN-R0706. However, no report about the effect of XAF-1407 in AF patients has been published up to date, so it remains unclear whether this compound would be efficient in clinical setting.

\section{Clinical potential of $\mathrm{I}_{\mathrm{SK}}$ inhibition for treatment of $\mathrm{AF}$}

The role of $\mathrm{I}_{\mathrm{SK}}$ in the electrical activity of the heart has been recognized relatively recently. ${ }^{68}$ Being first discovered in mammalian brain, ${ }^{69} \mathrm{SK}$ channels are widely expressed in neurons of central nervous system (CNS). This represents a major challenge for the development of SK channel inhibiting compounds for treatment of AF, since pharmacological inhibition of SK channels may potentially evoke adverse CNS-related effects. ${ }^{70}$ For example, NS8593, one of the first selective SK channel inhibitors and the predecessor of AP14145, was known to cause acute CNS effects. ${ }^{36}$ Similarly, in a pilot pharmacokinetic study with AP14145 we observed mild tremors in conscious goats. In order to develop safe SK channel inhibitors for treatment of AF, it is therefore essential that the SK channel inhibiting compounds do not pass the bloodbrain barrier and are efficient only in cardiac myocytes.

Recent developments of selective SK channel inhibitors resulted in the successor of AP14145, an SK channel inhibitor AP30663. ${ }^{71,72}$ This compound shows high atrial selectivity ${ }^{72}$ and the currently published studies report no CNS-related effects associated with its use. ${ }^{71,72}$ AP30663 showed promising results in rats and isolated guinea pig hearts ${ }^{71}$ as well as in pigs with AF, in which the compound was able to terminate vernakalant-resistant $\mathrm{AF}^{72}$ The first clinical study on AP30663 showed that the drug is safe and well-tolerated in humans, allowing further clinical testing. ${ }^{73}$ Upcoming clinical trials will indicate whether selective SK channel inhibition has a potential to become an antiarrhythmic strategy for treatment of AF.

\section{HOW DO ANTIARRHYTHMIC DRUGS TERMINATE AF?}

In preclinical studies, the effect of an antiarrhythmic agent is usually evaluated by assessment of various electrophysiological parameters, including APD, aERP or the slope of the action potential upstroke. ${ }^{35,36}$ Although changes in these parameters indicate potential antiarrhythmic effects of the drug, they have only a limited predictive value about the mechanism how the drug affects fibrillatory conduction. In fact, the changes in fibrillatory conduction pattern prior to AF termination have been documented only for a few antiarrhythmic drugs, ${ }^{74-76}$ leaving the information about the mechanisms of AF termination largely unexplored.

Performing high-resolution contact electrical mapping, chapter 2 and chapter 3 represent the first studies that described conduction patterns during AF termination with selective $\mathrm{I}_{\mathrm{KACh}}$ or $\mathrm{I}_{\mathrm{SK}}$ inhibiting drugs. For XAF-1407 as well as for AP14145, the cardioversion of AF to sinus 
rhythm was associated with abrupt changes in electrophysiological parameters just prior to AF termination, accompanied with sudden reduction of AF complexity and decrease in the number of fibrillatory waves. In contrast to these sudden changes, the prolongation of AFCL due to the administration of AADs was gradual. In cardioversion experiments in conscious goats in chapter 2 we observed that the AFCLs prior to AF cardioversion with vernakalant were significantly longer than the AFCLs preceding AF termination with XAF-1407. This finding suggests that the antiarrhythmic mechanisms of these drugs differ, with XAF-1407 exhibiting class III antiarrhythmic properties and with vernakalant predominantly having class I antiarrhythmic effect, as illustrated by previous studies investigating the effect of class I and class III AADs. ${ }^{74-77}$

The studies presented in chapter $\mathbf{2}$ and chapter $\mathbf{3}$ as well as numerous previous studies investigating the antiarrhythmic effects of $\mathrm{AADs}$ in the same goat model of $\mathrm{AF}^{11,20,44,77-80}$ suggest that prolongation of AFCL makes AF more likely to terminate. With progression of AF the complexity of atrial conduction increases, structural remodeling of atria occurs and the efficacy of pharmacological cardioversion decreases. ${ }^{11,57,58}$ A stronger effect of an AAD is therefore necessary to terminate AF, including prolongation of AFCL beyond the levels that were sufficient for cardioversion of AF at early stages of its progression (Figure 2). ${ }^{11}$ However, the AFCL at which AF cardioversion occurs largely depends on the mode of action of the AAD. ${ }^{77}$ In general, with class I AADs AF termination occurs at longer AFCL than with the class III AADs. ${ }^{77}$ Lengthening of AFCL therefore explains the mechanism of AF termination only partially, leaving the general mechanism underlying AF termination and the associated changes in electrophysiological parameters largely undescribed.

To obtain further insights into the mechanisms of AF termination, in chapter $\mathbf{4}$ we performed a retrospective analysis of bi-atrial high-density electrical mapping data from several studies in the goat model of pacing-induced AF. We demonstrated that cardioversion of AF to sinus rhythm was always preceded by an increased spatiotemporal organization of fibrillatory conduction during the last seconds before the arrhythmia termination. This process was accompanied with acceleration of atrial conduction and abrupt prolongation of AFCL, which is in agreement with previous studies investigating the process of AF cardioversion. ${ }^{79,81}$ Interestingly, these changes occurred irrespective of the presence or the type of AAD, indicating that sudden prolongation of AFCL and increased conduction velocity in atrial free walls preceded AF termination in goats in general. Investigating the conduction patterns before cardioversion, we found that the mechanism underlying AF termination was in most of the cases present outside of the atrial free walls. Our findings are highly suggestive that the site where the critical event underlying AF cardioversion occurred has been linked to anatomical structures or areas. 
Especially Bachmann's bundle might have played an important role in the last activation cycles preceding AF termination. However, to provide direct evidence for this hypothesis, more extensive mapping of atria would need to be performed.

A

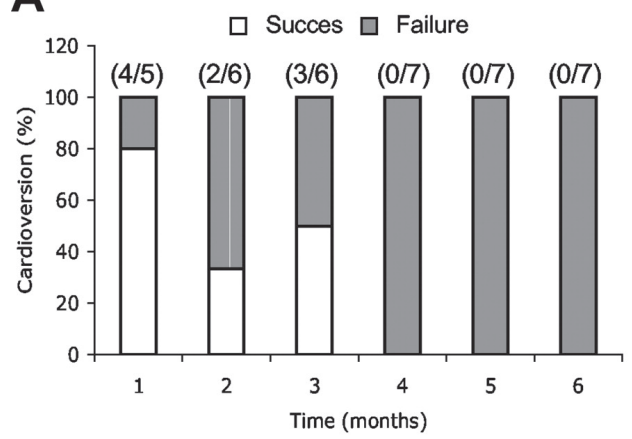

B

C
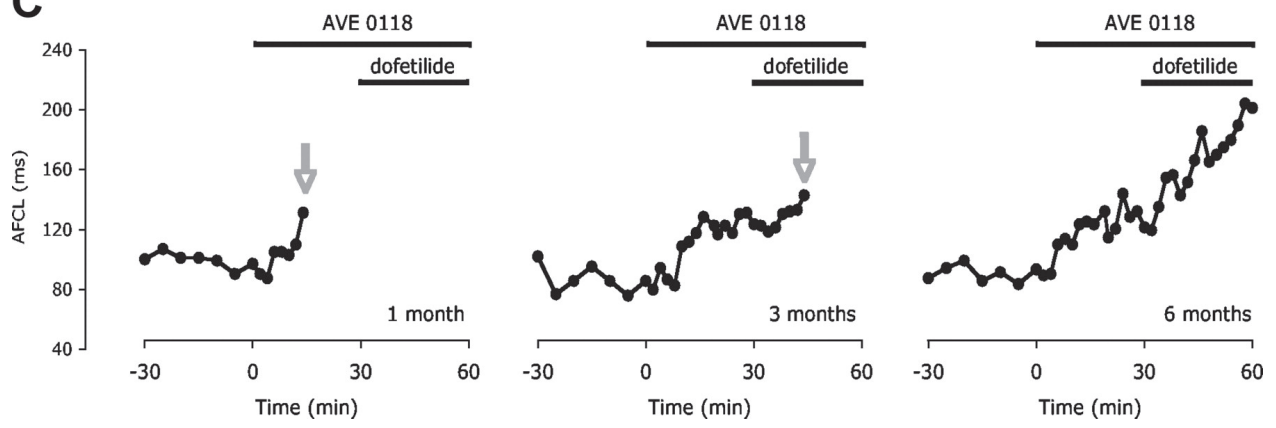

Figure 2. Decreasing efficacy of pharmacological cardioversion of atrial fibrillation (AF) in goats. (A) The success rate of pharmacological cardioversion with a combination of class III antiarrhythmic drugs AVE0118 and dofetilide decreased in time. (B) Baseline AF cycle length (AFCL) in conscious goats remained the same over the time course of six months. (C) Examples of AFCL prolongation when pharmacological cardioversion was attempted with the combination of AVE0118 and dofetilide. The examples illustrate that with progression of AF the AFCL preceding AF termination (indicated by an arrow) increases. At the time point of 6 months, the antiarrhythmic effect of AVE0118 and dofetilide was insufficient to terminate AF. Adapted with permission from Verheule et al., Copyright Wolters Kluwer Health Inc. ${ }^{11}$

The increased temporal organization of fibrillatory conduction in atrial free walls, manifested as a transition from continuous to periodic activity, was observed not only prior to AF termination, but we documented this process also during baseline AF. However, at baseline the intervals of periodic activity were always followed by the transition to continuous activity. The alterations between continuous and periodic activity occurring during AF do not have to be seen only as transient changes in organization of fibrillatory conduction. Considering 
that intervals of periodic activity always preceded cardioversion of AF, these transitions may be also interpreted as oscillations between phases with increased and decreased likelihood towards the arrhythmia termination. The study presented in chapter $\mathbf{4}$ cannot provide direct answer to the question whether the intervals of periodic activity are represented by conduction patterns that lead towards AF termination. To address this question, it would be necessary to investigate if similar activation patterns as those observed right before AF termination occurred at some earlier time point. Applying the methods of recurrent pattern analysis would therefore be a logical next step. ${ }^{82}$ It would allow to investigate whether the intervals of periodic and continuous activity are associated with a certain type of macroscopic pattern. Moreover, it could possibly identify whether AF termination is associated with a particular activation pattern, if such activation pattern is unique, or whether it occurred previously. Recurrent pattern analysis would therefore provide further insights into the mechanisms of AF cardioversion and could unravel the macroscopic mechanisms how AADs terminate AF.

The studies presented in chapters $\mathbf{2 - 4}$ investigated the antiarrhythmic effects of AADs in animals with electrically remodeled atria when the fibrillatory patterns are relatively simple and AF can be pharmacologically cardioverted. However, structural remodeling of the atria occurs with the progression of $\mathrm{AF}$, resulting in complex conduction patterns that make the arrhythmia more resistant to termination. Studying the mechanisms underlying perpetuation of AF in structurally remodeled atria thus has a potential to provide important knowledge about the reasons why the efficacy of currently available AADs decreases with the progression of the arrhythmia.

\section{STRUCTURAL REMODELING LEADS TO COMPLEX ATRIAL CONDUCTION}

The decline of cardioversion efficacy at late stages of AF is one of the major limitations of currently used AADs. ${ }^{80,83,84}$ With progression of the disease structural changes in atria develop, ${ }^{85-87}$ including atrial dilatation, ${ }^{56}$ fibrosis ${ }^{54,55}$ and myocyte hypertrophy. ${ }^{86}$ Especially atrial fibrosis is considered to play a pivotal role in AF substrate because it increases AF vulnerability ${ }^{54,55}$ and makes atrial conduction more complex. ${ }^{11,57,58}$ It is conceivable that some arrhythmogenic mechanisms become more pronounced in structurally remodeled atria. These mechanisms may include intramural reentry, ${ }^{88}$ longitudinal dissociation of excitation waves ${ }^{89}$ and loss of side-to-side coupling of atrial bundles that leads towards discontinuous conduction and zigzag activation patterns. ${ }^{90}$ Contributing to increased complexity of AF, these mechanisms make AF more stable and therefore resistant towards pharmacological cardioversion. ${ }^{11}$ 
To understand the reasons why currently used AADs fail to cardiovert complex AF and to facilitate the design of new pharmacological strategies, it is essential to have methods that allow in-depth investigation of complex conduction patterns occurring during AF. Electrical mapping is considered as a method of choice for studying activation patterns, mainly because it is easy to use and allows data acquisition in vivo. However, due to its physical nature, it has limited spatial resolution and is sensitive to far-field effects and interference from electrical stimulation. ${ }^{91}$ Optical mapping with voltage-sensitive dyes does not suffer from these limitations and represents therefore a more suitable tool for investigation of complex atrial conduction. ${ }^{88,92,93}$

In chapter 5 of this thesis, we presented a new method for detection of atrial activations in optical mapping recordings of AF. The method was designed to be highly sensitive to reveal small fibrillatory waves occurring during slow and discontinuous conduction. It is based on primary detection of atrial activations by sensitive template matching, which is an approach that has proved to be a powerful tool for identification of atrial activations in unipolar atrial electrograms of AF. ${ }^{94}$ The novelty of the presented method lies in the utilization of the nature how depolarization spreads through the cardiac tissue. Due to the propagating behavior of depolarization waves, true atrial activations occur in close proximity in both time and space. By applying spatiotemporal filtering of primarily detected activations, the method is capable to distinguish true atrial activations from false positive detections caused by residual noise that occur randomly in both space and time. As a consequence of that, the method performs well in identification of optical action potentials that are associated with slow, discontinuous conduction. Using a simple proof-of-principle model of two parallel myocyte strands, our group has previously shown that transverse conduction can behave differently from longitudinal conduction, including a different sensitivity to sodium and calcium current block. ${ }^{95}$ The presented method will allow investigation of the corresponding activation patterns and their sensitivity to AADs in intact atria.

In general, the method presented in chapter $\mathbf{5}$ has a potential to become a useful tool for investigation of complex activation patterns. It can provide new insights into the mechanisms underlying perpetuation of arrhythmias as well as into the mechanisms how AADs for rhythm control terminate AF. The method may facilitate studying discontinuous atrial conduction and zig-zag patterns that both have been identified as important arrhythmogenic mechanism in structurally remodeled atria. ${ }^{90,95,96}$ It can be considered as a first step for analysis of optical mapping recordings acquired during $\mathrm{AF}$, allowing identification and tracking of excitation waves. ${ }^{97}$ The usefulness of the method is illustrated in chapter $\mathbf{6}$ where it was further developed and used for analysis of the interactions between activation and repolarization patterns during 
AF. In such setting, it represents an essential part of a method that can be used as a tool for investigation of antiarrhythmic as well as possible proarrhythmic effects of AAD-induced prolongation of APD.

\section{ANTIARRHYTHMIC AND PROARRHYTHMIC EFFECTS OF ATRIAL APD PROLONGATION}

The efficacy of currently available AADs decreases with the progression of $\mathrm{AF}^{80,83,84}$ In order to terminate $\mathrm{AF}$ in later stages of the disease, much higher doses of AADs would be necessary. However, such doses cannot be safely administered in patients, because with increased plasma concentrations the atrial selectivity of the drugs decreases and potentially dangerous side effects on ventricular electrophysiology may occur. Lengthening of ventricular action potential, clinically manifested as prolongation of QT interval, is one of the most common effects on ventricular electrophysiology that the AADs for rhythm control can have. Being generally considered as proarrhythmic, QT prolongation may result in torsade de pointes or other type of ventricular tachycardia. ${ }^{98}$

In contrast to the prolongation of ventricular action potentials, lengthening of atrial action potentials is generally considered as antiarrhythmic. However, proarrhythmic effects related to prolonged atrial repolarization have been documented as well. It has been demonstrated that the use of antiarrhythmic agents may result in late phase 3 afterdepolarizations and reinduction of AF. ${ }^{99,100}$ Moreover, local heterogeneity in APD may lead to spatially discordant APD alternans and induction of reentry. ${ }^{101}$ Nevertheless, proarrhythmic mechanisms should not be reduced to changes in APD only. Triangulation of action potential, reversed use dependency, instability of APD and dispersion of refractoriness have been also identified to be proarrhythmic. ${ }^{98}$ The methods that would allow studying the spatial distribution of repolarization and APD thus have a potential to provide important insights about antiarrhythmic as well as possible proarrhythmic effects of AADs and may further unravel the mechanisms underlying perpetuation of AF. Electrical mapping of atrial electrograms which is probably the most common method for studying fibrillatory conduction during AF does not allow accurate determination of atrial repolarization patterns. By contrast, optical mapping in intact isolated hearts does allow recording of atrial repolarization, but analysis methods to map repolarization during fibrillatory conduction have not been developed extensively.

In chapter $\mathbf{6}$ we studied the interaction between patterns of atrial activation and repolarization in perfused hearts of goats with a history of AF. We demonstrated that during atrial pacing the repolarization patterns have a tendency to be synchronous over long distances while during AF atrial repolarization resembles the preceding activation pattern. The similarity of activation 
and repolarization patterns during AF favors the tendency of subsequent excitation waves to follow the same direction of propagation, known as 'linking of fibrillatory waves'. This mechanism is known to be associated with stabilization of functional reentry and perpetuation of arrhythmia. ${ }^{79,102,103}$

During atrial pacing the synchronized repolarization resulted in local prolongation of APD at the site of stimulation. This effect, confirmed by the results of a simple computer simulation, might have considerable implications for measurements of aERP. Considering a strong linear relationship between APD and aERP, ${ }^{104}$ our findings indicate that measurements of aERP by atrial pacing might be systematically overestimated when compared to the intrinsic APD at the site of pacing.

The method presented in chapter $\mathbf{6}$ offers a new possibility how to study repolarization patterns and can be used for studying arrhythmogenic mechanisms underlying perpetuation of atrial as well as ventricular arrhythmias. However, when investigating the repolarization processes in ventricles, three-dimensional behavior of repolarization should be considered due to the thickness of ventricular wall and the profound effect of intramural conduction. ${ }^{105}$ Studying the cardiac repolarization in the presence of AADs, the presented method has a potential to unravel the antiarrhythmic as well as potential proarrhythmic effects of these drugs, including early and late phase 3 afterdepolarizations and differences in dispersion of refractoriness and APD. ${ }^{98-100}$

Considering the low efficacy of clinically available AADs in later stages of AF development, a logical continuation of the work presented in chapter $\mathbf{6}$ would be to study atrial repolarization in structurally remodeled atria, when conduction patterns become complex and AF more difficult to terminate. Such investigation could provide important knowledge about the reasons why currently available $\mathrm{AADs}$ fail to terminate $\mathrm{AF}$ and could potentially contribute to the design of novel strategies for rhythm control therapy.

\section{CONCLUSION}

The EAST trial demonstrated that early initiation of rhythm control therapy is associated with better outcomes than usual care, encouraging the development of new pharmacological approaches for treatment of AF. The novel therapeutic targets investigated in this thesis, $\mathrm{I}_{\mathrm{KACh}}$ and $\mathrm{I}_{\mathrm{SK}}$, showed high atrial selectivity and efficacy to terminate the arrhythmia in the goat model of AF. Inhibition of these currents therefore has a potential to become a safe and efficient strategy for treatment of AF. Investigating the process of AF termination, we demonstrated that cardioversion of AF to sinus rhythm in goats is associated with abrupt prolongation of $\mathrm{AF}$ cycle length and acceleration of conduction velocity, resulting in decreased AF complexity. 
The lack of cardioversion efficacy of currently available AADs occurring at late stages of AF creates a need to study the mechanisms underlying perpetuation of the arrhythmia in structurally remodeled atria, when conduction patterns become complex and AF difficult to terminate. Exploiting the propagating behavior of cardiac depolarization, we developed a novel method for detection of atrial activations that is capable to identify small fibrillatory waves occurring during such complex fibrillatory conduction. Developing this method further, we provided an evidence showing that during AF the pattern of atrial repolarization is similar to the pattern of previous activation, which is a phenomenon that favors AF stability. These methods have a potential to be used for investigation of antiarrhythmic as well as possible proarrhythmic effects of AADs. They can facilitate development of new AADs that would be efficient to terminate AF, having increased ventricular safety at the same time. 


\section{REFERENCES}

1. Krijthe BP, Kunst A, Benjamin EJ, Lip GY, Franco OH, Hofman A, et al. Projections on the number of individuals with atrial fibrillation in the European Union, from 2000 to 2060. Eur Heart J 2013;34:2746-2751.

2. Hindricks G, Potpara T, Dagres N, Arbelo E, Bax JJ, Blomström-Lundqvist C, et al. 2020 ESC Guidelines for the diagnosis and management of atrial fibrillation developed in collaboration with the European Association of Cardio-Thoracic Surgery (EACTS). Eur Heart J 2020;42:373-498.

3. Wyse DG, Waldo AL, DiMarco JP, Domanski MJ, Rosenberg Y, Schron EB, et al. A comparison of rate control and rhythm control in patients with atrial fibrillation. N Engl J Med 2002;347:18251833.

4. Haïssaguerre M, Jaïs P, Shah DC, Takahashi A, Hocini M, Quiniou G, et al. Spontaneous initiation of atrial fibrillation by ectopic beats originating in the pulmonary veins. $N$ Engl J Med 1998;339:659-666.

5. Roy D, Talajic M, Nattel S, Wyse DG, Dorian P, Lee KL, et al. Rhythm control versus rate control for atrial fibrillation and heart failure. N Engl J Med 2008;358:2667-2677.

6. Hohnloser SH, Kuck KH, Lilienthal J. Rhythm or rate control in atrial fibrillation--Pharmacological Intervention in Atrial Fibrillation (PIAF): a randomised trial. Lancet 2000;356:1789-1794.

7. van Gelder IC, Hagens VE, Bosker HA, Kingma JH, Kamp O, Kingma T, et al. A comparison of rate control and rhythm control in patients with recurrent persistent atrial fibrillation. N EnglJ Med 2002;347:1834-1840.

8. Gillinov AM, Bagiella E, Moskowitz AJ, Raiten JM, Groh MA, Bowdish ME, et al. Rate Control versus Rhythm Control for Atrial Fibrillation after Cardiac Surgery. N Engl J Med 2016;374:19111921.

9. Waldo AL, Camm AJ, deRuyter H, Friedman PL, MacNeil DJ, Pauls JF, et al. Effect of d-sotalol on mortality in patients with left ventricular dysfunction after recent and remote myocardial infarction. The SWORD Investigators. Survival With Oral d-Sotalol. Lancet 1996;348:7-12.

10. Echt DS, Liebson PR, Mitchell LB, Peters RW, Obias-Manno D, Barker AH, et al. Mortality and morbidity in patients receiving encainide, flecainide, or placebo. The Cardiac Arrhythmia Suppression Trial. N Engl J Med 1991;324:781-788.

11. Verheule S, Tuyls E, van Hunnik A, Kuiper M, Schotten U, Allessie M. Fibrillatory conduction in the atrial free walls of goats in persistent and permanent atrial fibrillation. Circ Arrhythm Electrophysiol 2010;3:590-599.

12. Kirchhof P, Camm AJ, Goette A, Brandes A, Eckardt L, Elvan A, et al. Early Rhythm-Control Therapy in Patients with Atrial Fibrillation. N Engl J Med 2020;383:1305-1316.

13. Heijman J, Ghezelbash S, Dobrev D. Investigational antiarrhythmic agents: promising drugs in early clinical development. Expert Opin Investig Drugs 2017;26:897-907.

14. Ravens U, Odening KE. Atrial fibrillation: Therapeutic potential of atrial $\mathrm{K}^{+}$channel blockers. Pharmacol Ther 2017;176:13-21.

15. Li GR, Lau CP, Shrier A. Heterogeneity of sodium current in atrial vs epicardial ventricular myocytes of adult guinea pig hearts. J Mol Cell Cardiol 2002;34:1185-1194. 
16. Burashnikov A, Di Diego JM, Zygmunt AC, Belardinelli L, Antzelevitch C. Atrium-selective sodium channel block as a strategy for suppression of atrial fibrillation: differences in sodium channel inactivation between atria and ventricles and the role of ranolazine. Circulation 2007;116:14491457.

17. Kodama I, Kamiya K, Toyama J. Cellular electropharmacology of amiodarone. Cardiovasc Res 1997;35:13-29.

18. Varró A, Takács J, Németh M, Hála O, Virág L, Iost N, et al. Electrophysiological effects of dronedarone (SR 33589), a noniodinated amiodarone derivative in the canine heart: comparison with amiodarone. Br J Pharmacol 2001;133:625-634.

19. Fedida D, Orth PM, Chen JY, Lin S, Plouvier B, Jung G, et al. The mechanism of atrial antiarrhythmic action of RSD1235. J Cardiovasc Electrophysiol 2005;16:1227-1238.

20. Blaauw Y, Schotten U, van Hunnik A, Neuberger HR, Allessie MA. Cardioversion of persistent atrial fibrillation by a combination of atrial specific and non-specific class III drugs in the goat. Cardiovasc Res 2007;75:89-98.

21. Carstensen H, Kjær L, Haugaard MM, Flethøj M, Hesselkilde EZ, Kanters JK, et al. Antiarrhythmic Effects of Combining Dofetilide and Ranolazine in a Model of Acutely Induced Atrial Fibrillation in Horses. J Cardiovasc Pharmacol 2018;71:26-35.

22. Aguilar M, Xiong F, Qi XY, Comtois P, Nattel S. Potassium Channel Blockade Enhances Atrial Fibrillation-Selective Antiarrhythmic Effects of Optimized State-Dependent Sodium Channel Blockade. Circulation 2015;132:2203-2211.

23. Ni H, Whittaker DG, Wang W, Giles WR, Narayan SM, Zhang H. Synergistic Anti-arrhythmic Effects in Human Atria with Combined Use of Sodium Blockers and Acacetin. Front Physiol 2017;8:946.

24. Koumi S, Wasserstrom JA. Acetylcholine-sensitive muscarinic $\mathrm{K}^{+}$channels in mammalian ventricular myocytes. Am J Physiol 1994;266:H1812-H1821.

25. Noma A, Trautwein W. Relaxation of the ACh-Induced Potassium Current in the Rabbit Sinoatrial Node Cell. Pflügers Arch 1978;377:193-200.

26. Sakmann B, Noma A, Trautwein W. Acetylcholine activation of single muscarinic $\mathrm{K}^{+}$channels in isolated pacemaker cells of the mammalian heart. Nature 1983;303:250-253.

27. Milnes JT, Madge DJ, Ford JW. New pharmacological approaches to atrial fibrillation. Drug Discov Today 2012;17:654-659.

28. Krapivinsky G, Gordon EA, Wickman K, Velimirović B, Krapivinsky L, Clapham DE. The G-protein-gated atrial $\mathrm{K}^{+}$channel $\mathrm{I}_{\mathrm{KAch}}$ is a heteromultimer of two inwardly rectifying $\mathrm{K}^{+}$-channel proteins. Nature 1995;374:135-141.

29. Dobrev D, Friedrich A, Voigt N, Jost N, Wettwer E, Christ T, et al. The G protein-gated potassium current $\mathrm{I}(\mathrm{K}, \mathrm{ACh})$ is constitutively active in patients with chronic atrial fibrillation. Circulation 2005;112:3697-3706.

30. Skibsbye L, Poulet C, Diness JG, Bentzen BH, Yuan L, Kappert U, et al. Small-conductance calcium-activated potassium (SK) channels contribute to action potential repolarization in human atria. Cardiovasc Res 2014;103. 
31. Ling TY, Wang XL, Chai Q, Lau TW, Koestler CM, Park SJ, et al. Regulation of the SK3 channel by microRNA-499--potential role in atrial fibrillation. Heart Rhythm 2013;10:1001-1009.

32. Yu T, Deng C, Wu R, Guo H, Zheng S, Yu X, et al. Decreased expression of small-conductance $\mathrm{Ca}^{2+}$-activated $\mathrm{K}^{+}$channels SK1 and SK2 in human chronic atrial fibrillation. Life Sci 2012;90:219227.

33. Xia XM, Fakler B, Rivard A, Wayman G, Johnson-Pais T, Keen JE, et al. Mechanism of calcium gating in small-conductance calcium-activated potassium channels. Nature 1998;395:503-507.

34. Skibsbye L, Bengaard AK, Uldum-Nielsen AM, Boddum K, Christ T, Jespersen T. Inhibition of small conductance calcium-activated potassium (SK) channels prevents arrhythmias in rat atria during ß-adrenergic and muscarinic receptor activation. Front Physiol 2018;9:510.

35. Fenner MF, Carstensen H, Nissen SD, Hesselkilde EZ, Lunddahl C, Jensen MA, et al. Effect of selective $\mathrm{I}_{\mathrm{K}, \mathrm{Ach}}$ inhibition by XAF-1407 in an equine model of tachypacing-induced persistent atrial fibrillation. Br J Pharmacol 2020;177:3778-3794.

36. Simó-Vicens R, Kirchhof JE, Dolce B, Abilgaard L, Speerschneider T, Sorensen U, et al. A new negative allosteric modulator AP14145, for the study of small conductance calcium-activated potassium $\left(\mathrm{K}_{\mathrm{Ca}}\right.$ 2) channels. Br J Pharmacol 2017;174:4396-4408.

37. Diness JG, Skibsbye L, Simó-Vicens R, Santos JL, Lundegaard P, Citerni C, et al. Termination of vernakalant-resistant atrial fibrillation by inhibition of small-conductance $\mathrm{Ca}^{2+}$-activated $\mathrm{K}^{+}$ channels in pigs. Circ Arrhythm Electrophysiol 2017;10:e005125.

38. Haugaard MM, Hesselkilde EZ, Pehrson S, Carstensen H, Flethøj M, Præstegaard KF, et al. Pharmacologic inhibition of small-conductance calcium-activated potassium (SK) channels by NS8593 reveals atrial antiarrhythmic potential in horses. Heart Rhythm 2015;12:825-835.

39. Diness JG, Sørensen US, Nissen JD, Al-Shahib B, Jespersen T, Grunnet M, et al. Inhibition of small-conductance $\mathrm{Ca}^{2+}$-activated $\mathrm{K}^{+}$channels terminates and protects against atrial fibrillation. Circ Arrhythm Electrophysiol 2010;3:380-390.

40. Machida T, Hashimoto N, Kuwahara I, Ogino Y, Matsuura J, Yamamoto W, et al. Effects of a highly selective acetylcholine-activated $\mathrm{K}^{+}$channel blocker on experimental atrial fibrillation. Circ Arrhythm Electrophysiol 2011;4:94-102.

41. Yamamoto W, Hashimoto N, Matsuura J, Machida T, Ogino Y, Kobayashi T, et al. Effects of the Selective $\mathrm{K}_{\mathrm{ACh}}$ Channel Blocker NTC-801 on Atrial Fibrillation in a Canine Model of Atrial Tachypacing: Comparison With Class Ic and III Drugs. J Cardiovasc Pharmacol 2014;63:421-427.

42. Walfridsson H, Anderson KP, Anfinsen OG, Berggren A, Frison L, Jensen S, et al. Is the acetylcholine-regulated inwardly rectifying potassium current a viable antiarrhythmic target? Translational discrepancies of AZD2927 and A7071 in dogs and humans. Europace 2015;17:473482.

43. El-Haou S, Ford JW, Milnes JT. Novel K+ Channel Targets in Atrial Fibrillation Drug Development-Where Are We? J Cardiovasc Pharmacol 2015;66:412-431.

44. van Hunnik A, Lau DH, Zeemering S, Kuiper M, Verheule S, Schotten U. Antiarrhythmic effect of vernakalant in electrically remodeled goat atria is caused by slowing of conduction and prolongation of postrepolarization refractoriness. Heart Rhythm 2016;13:964-972. 
45. van Hunnik A, Nasrallah H, Lau DH, Kuiper M, Verheule S, Schotten U. Vernakalant does not alter early repolarization or contractility in normal and electrically remodelled atria. Europace 2018;20:140-148.

46. Mao ZL, Wheeler JJ, Clohs L, Beatch GN, Keirns J. Pharmacokinetics of novel atrial-selective antiarrhythmic agent vernakalant hydrochloride injection (RSD1235): influence of CYP2D2 expression and other factors. J Clin Pharmacol 2009;49:17-29.

47. Roy D, Pratt CM, Torp-Pedersen C, Wyse G, Toft E, Juul-Moller S, et al. Vernakalant hydrochloride for rapid conversion of atrial fibrillation: a phase 3 , randomized, placebo-controlled trial. Circulation 2008;117:1518-1525.

48. Camm AJ. The vernakalant story: how did it come to approval in Europe and what is the delay in the U.S.A? Curr Cardiol Rev 2014;10:309-314.

49. Citerni C, Kirchhoff J, Olsen LH, Sattler SM, Grunnet M, Edvardsson N, et al. Inhibition of $\mathrm{K}_{\mathrm{Ca}} 2$ and $\mathrm{K}_{\mathrm{v}} 11.1$ Channels in Pigs With Left Ventricular Dysfunction. Front Pharmacol 2020;11:556.

50. Lubberding AF, Sattler SM, Grunnet M, Sørensen US, Tfelt-Hansen J, Jespersen T. Arrhythmia development during inhibition of small-conductance calcium-activated potassium channels in acute myocardial infarction in a porcine model. Europace 2019;21:1584-1593.

51. Wijffels MCEF, Kirchhof CJHJ, Dorland R, Allessie MA. Atrial Fibrillation Begets Atrial Fibrillation: A Study in Awake Chronically Instrumented Goats. Circulation 1995;92:1954-1968.

52. Allessie M, Ausma J, Schotten U. Electrical, contractile and structural remodeling during atrial fibrillation. Cardiovasc Res 2002;54:230-246.

53. Schotten U, Verheule S, Kirchhof P, Goette A. Pathophysiological mechanisms of atrial fibrillation: a translational appraisal. Physiol Rev 2011;91:265-325.

54. Nattel S. Defining "culprit mechanisms" in arrhythmogenic cardiac remodeling. Circ Res 2004;94:1403-1405.

55. Verheule S, Sato T, Everett T, Engle SK, Otten D, Rubart-von der Lohe M, et al. Increased vulnerability to atrial fibrillation in transgenic mice with selective atrial fibrosis caused by overexpression of TGF-beta1. Circ Res 2004;94:1458-1465.

56. Shi Y, Ducharme A, Li D, Gaspo R, Nattel S, Tardif JC. Remodeling of atrial dimensions and emptying function in canine models of atrial fibrillation. Cardiovasc Res 2001;52:217-225.

57. Spach MS, Josephson ME. Initiating reentry: the role of nonuniform anisotropy in small circuits. J Cardiovasc Electrophysiol 1994;5:182-209.

58. Verheule S, Tuyls E, Gharaviri A, Hulsmans S, van Hunnik A, Kuiper M, et al. Loss of continuity in the thin epicardial layer because of endomysial fibrosis increases the complexity of atrial fibrillatory conduction. Circ Arrhythm Electrophysiol 2013;6:202-211.

59. Schoonderwoerd BA, van Gelder IC, van Veldhuisen DJ, Tieleman RG, Grandjean JG, Bel KJ, et al. Electrical remodeling and atrial dilation during atrial tachycardia are influenced by ventricular rate: role of developing tachycardiomyopathy. J Cardiovasc Electrophysiol 2001;12:1404-1410.

60. Guillemare E, Marion A, Nisato D, Gautier P. Inhibitory effects of dronedarone on muscarinic $\mathrm{K}^{+}$ current in guinea pig atrial cells. J Cardiovasc Pharmacol 2000;36:802-805.

61. Altomare C, Barbuti A, Viscomi C, Baruscotti M, DiFrancesco D. Effects of dronedarone on acetylcholine-activated current in rabbit SAN cells. Br J Pharmacol 2000;130:1315-1320. 
62. Watanabe Y, Hara Y, Tamagawa M, Nakaya H. Inhibitory effect of amiodarone on the muscarinic acetylcholine receptor-operated potassium current in guinea pig atrial cells. J Pharmacol Exp Ther 1996;279:617-624.

63. Voigt N, Rozmaritsa N, Trausch A, Zimniak T, Christ T, Wettwer E, et al. Inhibition of $I_{K, A C h}$ current may contribute to clinical efficacy of class I and class III antiarrhythmic drugs in patients with atrial fibrillation. Naunyn Schmiedebergs Arch Pharmacol 2010;381:251-259.

64. Kurachi Y, Nakajima T, Sugimoto T. Quinidine inhibition of the muscarine receptor-activated $\mathrm{K}^{+}$ channel current in atrial cells of guinea pig. Naunyn Schmiedebergs Arch Pharmacol 1987;335:216218.

65. Hara Y, Kizaki K. Antimalarial drugs inhibit the acetylcholine-receptor-operated potassium current in atrial myocytes. Heart Lung Circ 2002;11:112-116.

66. Fedida D. Vernakalant (RSD1235): a novel, atrial-selective antifibrillatory agent. Expert Opin Investig Drugs 2007;16:519-532.

67. Podd SJ, Freemantle N, Furniss SS, Sulke N. First clinical trial on specific $I_{\text {KACh }}$ blocker shows no reduction in atrial fibrillation burden in patients with paroxysmal atrial fibrillation: pacemaker assessment of BMS 914392 in patients with paroxysmal atrial fibrillation. Europace 2016;18:340346.

68. Diness JG, Bentzen BH, Sørensen US, Grunnet M. Role of Calcium-activated Potassium Channels in Atrial Fibrillation Pathophysiology and Therapy. J Cardiovasc Pharmacol 2015;66:441-448.

69. Köhler M, Hirschberg B, Bond CT, Kinzie JM, Marrion NV, Maylie J, et al. Small-conductance, calcium-activated potassium channels from mammalian brain. Science 1996;273:1709-1714.

70. Lallement G, Fosbraey P, Baille-Le-Crom V, Tattersall JE, Blanchet G, Wetherell JR, et al. Compared toxicity of the potassium channel blockers, apamin and dendrotoxin. Toxicology 1995;104:47-52.

71. Bentzen BH, Bomholtz SH, Simó-Vicens R, Folkersen L, Abildgaard L, Speerschneider T, et al. Mechanisms of Action of the $\mathrm{K}_{\mathrm{Ca}} 2$-Negative Modulator AP30663, a Novel Compound in Development for Treatment of Atrial Fibrillation in Man. Front Pharmacol 2020;11:610.

72. Diness JG, Kirchhoff JE, Speerschneider T, Abildgaard L, Edvardsson N, Sørensen US, et al. The $\mathrm{K}_{\mathrm{Ca}} 2$ Channel Inhibitor AP30663 Selectively Increases Atrial Refractoriness, Converts VernakalantResistant Atrial Fibrillation and Prevents Its Reinduction in Conscious Pigs. Front Pharmacol 2020;11:159.

73. Gal P, Klaassen ES, Bergmann KR, Saghari M, Burggraaf J, Kemme MJB, et al. First Clinical Study with AP30663 - a $\mathrm{K}_{\mathrm{Ca}} 2$ Channel Inhibitor in Development for Conversion of Atrial Fibrillation. Clin Transl Sci 2020;13:1336-1344.

74. Wang Z, Pagé P, Nattel S. Mechanism of flecainide's antiarrhythmic action in experimental atrial fibrillation. Circ Res 1992;71:271-287.

75. Wang J, Bourne GW, Wang Z, Villemaire C, Talajic M, Nattel S. Comparative mechanisms of antiarrhythmic drug action in experimental atrial fibrillation. Importance of use-dependent effects on refractoriness. Circulation 1993;88:1030-1044.

76. Wang J, Feng J, Nattel S. Class III antiarrhythmic drug action in experimental atrial fibrillation. Differences in reverse use dependence and effectiveness between $\mathrm{d}$-sotalol and the new antiarrhythmic drug ambasilide. Circulation 1994;90:2032-2040. 
77. Wijffels MCEF, Dorland R, Allessie MA. Pharmacologic Cardioversion of Chronic Atrial Fibrillation in the Goat by Class Ia, Ic and III Drugs: A Comparison Between Hydroquinidine, Cibenzoline, Flecainide and d-Sotalol. J Cardiovasc Electrophysiol 1999;10:178-193.

78. Blaauw Y, Gogelein H, Tieleman RG, van Hunnik A, Schotten U, Allessie MA. "Early" class III drugs for the treatment of atrial fibrillation: efficacy and atrial selectivity of AVE0118 in remodeled atria of the goat. Circulation 2004;110:1717-1724.

79. Shan Z, van der Voort PH, Blaauw Y, Duytschaever M, Allessie MA. Fractionation of electrograms and linking of activation during pharmacologic cardioversion of persistent atrial fibrillation in the goat. J Cardiovasc Electrophysiol 2004;15:572-580.

80. Eijsbouts S, Ausma J, Blaauw Y, Schotten U, Duytschaever M, Allessie MA. Serial cardioversion by class IC drugs during 4 months of persistent atrial fibrillation in the goat. J Cardiovasc Electrophysiol 2006;17:648-654.

81. de Brito Santos PE, Duytschaever M, Allessie MA. Low-frequency oscillations of atrial fibrillation cycle length in goats: characterization and potentiation by class III antiarrhytmic almokalant. J Electrocardiol 2008;41:711-723.

82. van Hunnik A, Zeemering S, Podziemski P, Simons J, Gatta G, Hannink L, et al. Stationary Atrial Fibrillation Properties in the Goat Do Not Entail Stable or Recurrent Conduction Patterns. Front Physiol 2018;9:947.

83. Goy JJ, Kaufmann U, Kappenberger L, Sigwart U. Restoration of sinus rhythm with flecainide in patients with atrial fibrillation. Am J Cardiol 1988;62:38d-40d.

84. Frick M, Frykman V, Jensen-Urstad M, Ostergren J, Rosenqvist M. Factors predicting success rate and recurrence of atrial fibrillation after first electrical cardioversion in patients with persistent atrial fibrillation. Clin Cardiol 2001;24:238-244.

85. Ausma J, Litjens N, Lenders MH, Duimel H, Mast F, Wouters L, et al. Time course of atrial fibrillation-induced cellular structural remodeling in atria of the goat. J Mol Cell Cardiol 2001;33:2083-2094.

86. Ausma J, Wijffels MCEF, Thoné F, Wouters L, Allessie M, Borgers M. Structural changes of atrial myocardium due to sustained atrial fibrillation in the goat. Circulation 1997;96:3157-3163.

87. Boldt A, Wetzel U, Lauschke J, Weigl J, Gummert J, Hindricks G, et al. Fibrosis in left atrial tissue of patients with atrial fibrillation with and without underlying mitral valve disease. Heart 2004;90:400-405.

88. Hansen BJ, Zhao J, Csepe TA, Moore BT, Li N, Jayne LA, et al. Atrial fibrillation driven by microanatomic intramural re-entry revealed by simultaneous sub-epicardial and sub-endocardial optical mapping in explanted human hearts. Eur Heart J 2015;36:2390-2401.

89. Allessie MA, de Groot NM, Houben RP, Schotten U, Boersma E, Smeets JL, et al. Electropathological substrate of long-standing persistent atrial fibrillation in patients with structural heart disease: longitudinal dissociation. Circ Arrhythm Electrophysiol 2010;3:606-615.

90. Spach MS, Boineau JP. Microfibrosis produces electrical load variations due to loss of side-toside cell connections: a major mechanism of structural heart disease arrhythmias. Pacing Clin Electrophysiol 1997;20:397-413. 
91. Herron TJ, Lee P, Jalife J. Optical imaging of voltage and calcium in cardiac cells \& tissues. Circ Res 2012;110:609-623.

92. Mandapati R, Skanes A, Chen J, Berenfeld O, Jalife J. Stable Microreentrant Sourcees as a Mechanism of Atrial Fibrillation in the Isolated Sheep Heart. Circulation 2000;101:194-199.

93. Verheule S, Wilson E, Banthia S, Everett TH, Shanbhag S, Sih HJ, et al. Direction-dependent conduction abnormalities in a canine model of atrial fibrillation due to chronic atrial dilatation. $\mathrm{Am}$ J Physiol Heart Circ Physiol 2004;287:H634-H644.

94. Zeemering S, Maesen B, Nijs J, Lau DH, Granier M, Verheule S, et al. Automated quantification of atrial fibrillation complexity by probabilistic electrogram analysis and fibrillation wave reconstruction. Conf Proc IEEE Eng Med Biol Soc 2012;2012:6357-6360.

95. Zhao J, Schotten U, Smaill B, Verheule S. Loss of Side-to-Side Connections Affects the Relative Contributions of the Sodium and Calcium Current to Transverse Propagation Between Strands of Atrial Myocytes. Front Physiol 2018;9:1212.

96. Koura T, Hara M, Takeuchi S, Ota K, Okada Y, Miyoshi S, et al. Anisotropic conduction properties in canine atria analyzed by high-resolution optical mapping: preferential direction of conduction block changes from longitudinal to transverse with increasing age. Circulation 2002;105:20922098.

97. Tomek J, Burton RAB, Bub G. Ccoffinn: Automated Wave Tracking in Cultured Cardiac Monolayers. Biophys J 2016;111:1595-1599.

98. Shah RR, Hondeghem LM. Refining detection of drug-induced proarrhythmia: QT interval and TRIaD. Heart Rhythm 2005;2:758-772.

99. Burashnikov A, Antzelevitch C. Reinduction of atrial fibrillation immediately after termination of the arrhythmia is mediated by late phase 3 early afterdepolarization-induced triggered activity. Circulation 2003;107:2355-2360.

100. Burashnikov A, Antzelevitch C. Late-phase 3 EAD. A unique mechanism contributing to initiation of atrial fibrillation. Pacing Clin Electrophysiol 2006;29:290-295.

101. Weiss JN, Karma A, Shiferaw Y, Chen PS, Garfinkel A, Qu Z. From pulsus to pulseless: the saga of cardiac alternans. Circ Res 2006;98:1244-1253.

102. Gerstenfeld EP, Sahakian AV, Swiryn S. Evidence for transient linking of atrial excitation during atrial fibrillation. Circulation 1992;86:375-382.

103. Schoenwald AT, Sahakian AV, Sih HJ, Swiryn S. Further observations of "linking" of atrial excitation during clinical atrial fibrillation. Pacing Clin Electrophysiol 1998;21:25-34.

104. Bode F, Kilborn M, Karasik P, Franz MR. The repolarization-excitability relationship in the human righ atrium is unaffected by cycle length, recording site and prior arrhythmias. J Am Coll Cardiol 2001;37:920-925.

105. Christoph J, Chebbok M, Richter C, Schröder-Schetelig J, Bittihn P, Stein S, et al. Electromechanical vortex filaments during cardiac fibrillation. Nature 2018;555:667-672. 



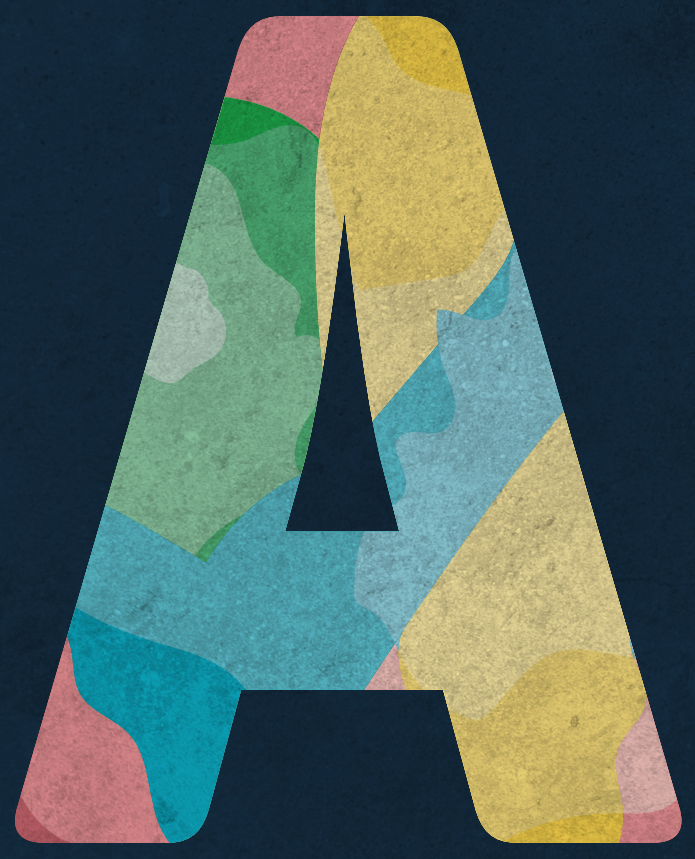




\section{Appendix}

Summary

Shrnutí

Impact paragraph

Acknowledgments

About the author 



\section{SUMMARY}

Atrial fibrillation (AF) is a rhythm disorder characterized by fast and irregular activations of the atria. Being a major cause of stroke, heart failure and overall cardiovascular morbidity, AF represents a considerable challenge for public health and a significant socioeconomic burden. In many patients the AF episodes are asymptomatic and terminate spontaneously. However, due to the progressive behavior of the disease the episodes prolong until AF becomes persistent. Early diagnosis and effective treatment of AF are therefore essential for successful clinical management of the arrhythmia.

The recently published Early treatment of Atrial fibrillation for Stroke prevention Trial (EAST) showed that early treatment of AF by antiarrhythmic drugs (AADs) or by catheter ablation slows down the progression of the arrhythmia. However, neither of the strategies achieves satisfying success rates in the later stages of the disease. Moreover, the use of currently available AADs is limited by their side effects, especially by an increased risk of development of druginduced ventricular arrhythmias. This creates an urgent need for development of new AADs that would be safe and efficient at the same time, as well as for better understanding of the mechanisms that are responsible for perpetuation of AF. This thesis aims to provide new insights in both fields. We investigated the potential of pharmacological inhibition of two ion currents that are both considered as promising targets for treatment of AF. In addition, we studied the mechanisms of AF termination under various conditions. Finally, we present a new analysis tool for studying complex atrial conduction and the interaction between activation and repolarization waves during both pacing and $\mathrm{AF}$.

In chapter 2 of this thesis we studied the antiarrhythmic effect of a highly specific acetylcholineactivated potassium current $\left(\mathrm{I}_{\mathrm{KACh}}\right)$ inhibitor XAF-1407 in a goat model of pacing-induced AF. We demonstrated that XAF-1407 is a potent AAD for AF termination in the goat. The administration of XAF-1407 was not associated with any ventricular arrhythmias and its effect on ventricular electrophysiology was only limited. Pharmacological inhibition of $\mathrm{I}_{\mathrm{KACh}}$ by XAF-1407 resulted in more pronounced prolongation of atrial effective refractory period in electrically remodeled atria than in normal atria. This observation agrees with the hypothesis that the potency of $\mathrm{I}_{\mathrm{KACh}}$ inhibition may be increased in electrically remodeled atria due to a constitutively active $\mathrm{I}_{\mathrm{KACh}}$. Our findings indicate that $\mathrm{I}_{\mathrm{KACh}}$ inhibition has a potential to become not only atrial-selective, but also AF-selective strategy for treatment of AF, being safe in the ventricles at the same time.

Chapter 3 investigates the antiarrhythmic properties of a small conductance calcium-activated potassium current $\left(\mathrm{I}_{\mathrm{SK}}\right)$ inhibitor AP14145, again in a goat model of pacing-induced AF. Showing that AP14145 is able to terminate persistent AF in goats, the study demonstrates that 
AP14145 is an efficient antiarrhythmic agent for AF cardioversion. Rate-dependent reduction of conduction velocity observed during atrial pacing indicated a possible class I effect of the $I_{S K}$ inhibition. The administration of AP14145 was not associated with any changes in ventricular electrophysiology, neither with ventricular proarrhythmic effects. Pharmacological inhibition of $\mathrm{I}_{\mathrm{SK}}$ might therefore also represent a safe therapeutic strategy for treatment of AF. The AF terminations in presence of AP14145 were preceded by gradual prolongation of AF cycle length. However, to our surprise, the decrease of AF complexity before cardioversion was sudden, occurring just in the last AF cycles before the arrhythmia termination.

To investigate the process of AF termination more in depth, in chapter $\mathbf{4}$ we performed a retrospective analysis of several studies in the goat model of pacing-induced AF, including the studies presented in chapter 2 and chapter 3. We analyzed bi-atrial high-density electrical mapping data that were acquired during pharmacologically-induced as well as spontaneous AF terminations. The study demonstrates that cardioversion of AF was always preceded by increased spatiotemporal organization of fibrillatory conduction during the final seconds before the arrhythmia termination. This process was associated with abrupt changes in electrophysiological parameters. Our findings are highly suggestive that conduction through Bachmann's bundle might play an important role in the last activation cycles before AF termination.

The studies presented in chapters $\mathbf{2 - 4}$ investigated the antiarrhythmic effects of AADs in animals with electrically remodeled atria when the fibrillatory patterns were relatively simple and AF could be pharmacologically cardioverted. With the progression of AF however, more complex conduction patterns occur and the arrhythmia is more resistant to termination. To understand the reasons why currently used AADs fail to cardiovert complex AF, it is essential to have methods that allow in-depth investigation of complex conduction patterns. Optical mapping proved to be a useful tool for exploration of the mechanisms driving cardiac arrhythmias because it allows recording of optical action potentials with high spatial and temporal resolution.

Chapter 5 presents a novel method that is tailored for detection of atrial activations in optical mapping recordings of AF. The method was designed to be highly sensitive to reveal small fibrillatory waves that occur during complex atrial conduction. The study demonstrates that the method is capable to identify optical action potentials with low magnitude and slow upstroke that are associated with slow, discontinuous conduction. The method can be considered as a first step for further analysis of optical mapping recordings acquired during cardiac fibrillation, allowing advanced analysis of activation patterns and identification and tracking of excitation waves. 
Developing further the above-mentioned method, chapter 6 investigates the interaction between atrial activation and repolarization patterns in perfused hearts of goats with persistent AF. It demonstrates that atrial repolarization during pacing shows rather synchronous behavior, resulting in local prolongation of action potential duration at the site of stimulation. In contrast, during AF atrial repolarization shows wave-like propagating behavior with a pattern that resembles the preceding activation. This similarity favors the tendency of subsequent excitation waves to follow the same direction of propagation, which is a phenomenon known as 'linking' of fibrillatory waves. The close resemblance of activation and repolarization patterns during AF therefore facilitates stabilization of functional reentry and perpetuation of the arrhythmia.

Taken together, the work presented in this thesis describes the antiarrhythmic mechanisms of novel AADs, showing that inhibition of $\mathrm{I}_{\mathrm{KACh}}$ and $\mathrm{I}_{\mathrm{SK}}$ has a potential to become safe and efficient therapeutic strategy for AF cardioversion. The work provides new insights into the mechanisms underlying $\mathrm{AF}$ termination, demonstrating that regardless of the presence or the type of $\mathrm{AAD}$, cardioversion of $\mathrm{AF}$ in goats is associated with increased organization of the fibrillatory process and with abrupt changes in electrophysiological parameters. Applying a new method for analysis of optical mapping data, the work also provides new insights into the mechanisms underlying perpetuation of the arrhythmia, showing that during AF the pattern of atrial repolarization tends to resemble the pattern of preceding activation. 



\section{SHRNUTí}

Fibrilace síní (FS) je porucha srdečního rytmu, která se vyznačuje rychlými a nepravidelnými aktivacemi srdečních síní. Přítomnost FS je spojena s významným výskytem cévních mozkových př́hod a srdečního selhání. V porovnání s pacienty se sinusovým rytmem je úmrtnost pacientů s FS dvojnásobná. Z těchto důvodů představuje léçba FS významnou klinickou, ale také socioekonomickou zátěž. Část pacientů s FS je asymptomatická a arytmické epizody u nich končí spontánně. Progresivní charakter onemocnění však způsobuje, že epizody se časem prodlužují, a to až do okamžiku, kdy FS přetrvává (je perzistentní). V̌casná diagnóza a efektivní možnosti terapie jsou proto pro klinické zvládání této arytmie zcela zásadní.

Nedávno zveřejněná klinická studie EAST (Early treatment of Atrial fibrillation for Stroke prevention Trial) ukázala, že včasná léčba FS pomocí antiarytmik nebo pomocí katetrizační ablace zpomaluje progresi onemocnění. Úspěšnost těchto metod v pozdních stadiích FS však není uspokojivá. Použití antiarytmik je navíc do značné míry limitováno vedlejšími účinky, zvláště pak zvýšeným rizikem vzniku komorových arytmií. To způsobuje potřebu vyvinout účinná a zároveň bezpečná antiarytmika, stejně tak jako nutnost lepšího porozumění mechanismům, které pomáhají FS udržovat. Tato práce si klade za cíl poskytnout nové poznatky v obou těchto oblastech. Práce zkoumá možnosti farmakologické inhibice dvou iontových proudů, které významně ovlivňují průběh akčního potenciálu a které se vyskytují převážně v síňovém myokardu. Dále se pak práce věnuje studiu mechanismů, které předcházejí konci arytmických epizod. Poslední kapitoly pak popisují nově vyvinutou metodu pro studium síňového vedení v průběhu FS a interakci mezi depolarizačními a repolarizačními vlnami.

Kapitola 2 studuje antiarytmické účinky látky XAF-1407, která je vysoce specifickým inhibitorem acetylcholinem-aktivovaného draselného proudu $\left(\mathrm{I}_{\mathrm{KACh}}\right)$. Pro účely této studie byly použity kozy, u kterých byla FS vyvolána chronickou stimulací síní. V této kapitole je ukázáno, že XAF-1407 je na daném animálním modelu vysoce účinné antiarytmikum. Nitrožilní podání XAF-1407 nevedlo ke komorovým arytmiím a efekt této látky na elektrofyziologii srdečních komor byl pouze minimální. Podáním XAF-1407 došlo k prodloužení refrakterní periody síní. Tento efekt byl výraznější v síních po elektrické remodelaci. Uvedené poznatky jsou v souladu s hypotézou, že inhibice $\mathrm{I}_{\mathrm{KACh}}$ má výraznější efekt v elektricky remodelovaných síních z důvodu konstitutivně aktivního $I_{\mathrm{KACh}}$. Naše výsledky ukazují, že farmakologická inhibice $\mathrm{I}_{\mathrm{KACh}}$ má síňově selektivní a zároveň FS-selektivní efekt a má tak potenciál stát se bezpečnou strategií pro léčbu FS.

Kapitola 3 popisuje studii, která zkoumá antiarytmické vlastnosti látky AP14145, což je inhibitor vápníkem aktivovaných draselných proudů o nízké vodivosti $\left(\mathrm{I}_{\mathrm{SK}}\right)$. Studie byla opět provedena na kozách, u kterých byla FS vyvolána chronickou stimulací srdečních síní. Kapitola 
ukazuje, že AP14145 je efektivním antiarytmikem pro kardioverzi FS. U koz bylo pozorováno snižování rychlosti síňového vedení v průběhu elektrické stimulace v závislosti na frekvenci stimulace. Tento poznatek poukazuje na možné účinky inhibice $\mathrm{I}_{\mathrm{SK}}$ odpovídající antiarytmické třídě I. Nitrožilní podání AP14145 nebylo provázeno změnami v elektrofyziologii komor, ani proarytmickými událostmi. Podobně jako inhibice $\mathrm{I}_{\mathrm{KACh}}$ může také farmakologická inhibice $\mathrm{I}_{\mathrm{SK}}$ představovat bezpečnou a účinnou strategii pro léčbu FS. Kardioverzi FS za př́tomnosti AP14145 předcházelo postupné prodlužování času mezi jednotlivými fibrilačními cykly. Naproti tomu pokles ve složitosti síňového vedení byl náhlý a objevoval se pouze v průběhu posledních cyklů předcházejících konci FS.

Za účelem podrobnějšího zkoumání procesů, které předcházejí konci arytmických epizod jsme v kapitole 4 provedli retrospektivní analýzu několika studií provedených na již zmíněném kozím modelu FS. Tato analýza mimo jiné zahrnovala i data z kapitol 2 a 3. Pro účely studie jsme použili data získaná elektrickým mapování síní, které bylo provedeno při farmakologicky indukovaných i spontánních kardioverzích za použití mapovacích elektrod umístěných epikardiálně na obou síních. Kapitola ukazuje, že kardioverze FS byla vždy předcházena zvýšenou časovou i prostorovou organizací síňového vedení v průběhu posledních vteřin préed skončením arytmie. Tento proces byl doprovázen náhlými změnami elektrofyziologických parametrů popisujících síňové vedení. Naše poznatky naznačují, že vedení Bachmannovým svazkem může hrát důležitou roli v průběhu posledních aktivačních cyklů předcházejících konci FS a přechodu k sinusovému rytmu.

V kapitolách 2-4 byly studovány antiarytmické vlastnosti léků a preparátů u koz s elektricky remodelovanými síněmi. U těchto zvířat byla FS ve stadiu, které umožňovalo farmakologickou kardioverzi. Progrese FS nicméně vede $\mathrm{k}$ postupně složitějšímu síňovému vedení, což má za následek vyšší rezistenci arytmie k farmakologické kardioverzi. Pro lepší pochopení, proč současná antiarytmika selhávají při kardioverzi FS v jejích pozdějších stádiích, je důležité mít k dispozici metody, které umožňují detailní studium složitého síňového vedení. Optické mapování se ukázalo jako velice užitečný nástroj pro zkoumání mechanismů způsobujících srdeční arytmie, nebot umožňuje zaznamenávání optických akčních potenciálů s vysokým prostorovým i časovým rozlišením.

V kapitole 5 je představena nová metoda pro detekci aktivačních časů v záznamech optického mapování pořízených při FS. Tato metoda byla navržena tak, aby umožňovala odhalit malé aktivační vlny, které se vyskytují v průběhu složitého síňového vedení typického pro pozdní fáze FS. Uvedená metoda je schopna identifikovat optické akční potenciály s nízkou amplitudou a pomalou depolarizací, které se vyskytují při nesouvislém síňovém vedení. Tato metoda může 
být použita jako první krok pro analýzu záznamů pořizených pomocí optického mapování, které zachycují srdeční fibrilace, a může tak umožnit pokročilou analýzu aktivačních vzorců a identifikaci a trasování excitačních vln.

Metoda představená v kapitole 5 byla dále rozvinuta a použita v kapitole 6, která popisuje interakci mezi aktivačními a repolarizačními sekvencemi v perfundovaných srdcích koz s persistentní FS. Tato kapitola ukazuje, že repolarizace má při elektrické stimulaci síní převážně synchronní charakter, který zpưsobuje lokální prodloužení akčních potenciálů v místě stimulace. Naproti tomu při FS má repolarizace převážně charakter vlny, jejíž směr a rychlost šíření se podobají předchozí depolarizační vlně. Tato vlastnost síňové repolarizace způsobuje, že při FS se jednotlivé excitační vlny šírí stejným směrem, což je známo jako fenomén ,spojených ‘ fibrilačních vln. Podobnost aktivačních a repolarizačních sekvencí v průběhu FS tak napomáhá stabilizaci funkčního reentry a udržování arytmie.

Celkově vzato, tato práce popisuje mechanismy působení nových antiarytmik a ukazuje, že farmakologická inhibice $\mathrm{I}_{\mathrm{KACh}}$ a $\mathrm{I}_{\mathrm{SK}}$ může být potenciálně bezpečnou a efektivní strategií pro léčbu FS. Zároveň práce poskytuje nové poznatky o mechanismech, které předcházejí samotné kardioverzi FS. Ukazuje, že nehledě na př́tomnost nebo typ antiarytmika, kardioverze FS je u koz doprovázena zvýšenou organizací síňového vedení a náhlými změnami elektrofyziologických parametrů srdečních síní. Za pomoci nových nástrojů pro analýzu záznamů z optického mapování tato práce poskytuje nové poznatky o mechanismech, které se podílejí na udržování arytmie, nebot̀ ukazuje, že při FS má repolarizace tendenci následovat směr šiření předchozí aktivace. 



\section{IMPACT PARAGRAPH}

Being the most common arrhythmia in clinical practice, atrial fibrillation (AF) represents a major challenge for public health. Aging of the population that is expected in the coming decades will result in substantial increase in the number of patients with AF. Having effective options for AF treatment is therefore essential. Currently available therapeutic approaches for management of AF are insufficient. The use of antiarrhythmic drugs (AADs) that are available for AF treatment is limited by severe side effects that can be potentially lethal. Therefore, there is an urgent need for development of new AADs as well as for better understanding of the mechanisms that are underlying the maintenance and termination of AF.

In this thesis, we studied new approaches for treatment of AF by AADs. We investigated two compounds, each working on a different principle, testing them in goats with AF. Our results demonstrate that both therapeutic approaches have a potential to become safe and efficient strategies for AF treatment. This finding can support development of new drugs that would work on the same or very similar principle, leading to improvement of the available pharmacological approaches for treatment of AF.

Performing a retrospective analysis of data acquired during AF terminations in goats, we demonstrated that cardioversion of AF in goats is associated with sudden changes in atrial electrophysiology during the last $\mathrm{AF}$ cycles before the arrhythmia termination, regardless of the presence or the type of AAD. This finding provides new insights into the mechanisms that lead to AF cardioversion and improves our understanding about the process of AF termination itself. In long-term perspective, this knowledge may facilitate the design of new approaches for treatment or prevention of AF.

To improve the current pharmacological strategies for treatment of AF, it is necessary to develop AADs that would be efficient in patients with long-standing complex AF and safe in patients with underlying structural heart disease that are at increased risk of developing severe adverse events. The new method for identification of small fibrillatory waves that is presented in this thesis will facilitate studying of complex atrial conduction. It has a potential to improve our understanding why AADs lose their efficacy when AF becomes complex and difficult to terminate. By further development of this method, we showed that during AF the pattern of atrial repolarization resembles the pattern of previous activation, which is a phenomenon that favors AF stability. The methods developed in this thesis may facilitate studying of antiarrhythmic as well as possible proarrhythmic effects of AADs. From this perspective, the presented tools can be used for improvement of current pharmacological strategies for treatment of AF. 



\section{ACKNOWLEDGMENTS}

Although there is only one name stated on the cover page, there are many people without whom this book would have never been created. All together, they form an amazing mosaic of human beings who supported me throughout my journey. With the lines below, I would like to express my gratitude to those who directly contributed to this work, as well as to the ones who were supporting me by being my friends or just by being around.

First of all, I would like to express my gratitude to Prof. Schotten. Dear Uli, thank you so much for giving me the opportunity of becoming a member of your research group. Thanks to you, I could have spent some unforgettable years in Maastricht and at the Department of Physiology. It was very inspiring to follow your ideas and to see how you approach various scientific problems. Our meetings often showed me new perspectives on how to tackle the challenges a $\mathrm{PhD}$ student has to deal with. It is very difficult to imagine this work being done without your input.

This thesis would have never been possible without my daily supervisor Dr. Verheule. Dear Sander, I'm very grateful that it was you who guided me through my doctoral studies. I know that especially in the very first months I wasn't the easiest person to work with. However, you dealt with all the problems we faced together with a calm mind and great expertise, which are qualities that I truly appreciate and admire. It has been an honor to be your PhD student and to get scientifically shaped by you. Thank you for sharing all your experience and wide knowledge with me. I have learned so much from you!

My gratitude also goes to Dr. van Hunnik. Dear Arne, thank you for your support from the very beginning of my $\mathrm{PhD}$ trajectory. I cannot imagine performing all the experimental work without your guidance and advice. You are in possession of a true scientific mind and I am happy that I had the opportunity to discuss with you various scientific and also non-scientific topics. It was nice to work with you, be it in the lab, in the stables or behind computer. Thank you for your innumerable inputs into my work. I believe our collaboration will remain recurrent and it will take long before it terminates.

I would also like to thank to the members of the Assessment Committee Prof. Frits Prinzen, Prof. Marc Vos, Dr. Matthijs Blankesteijn and Dr. Ruben Coronel for reviewing my thesis.

My sincere thanks go to Dr. Zeemering, a great colleague and the programming guru of the group. Dear Stef, thank you so much for all your help and the time you spent by explaining 
me why my code does not work. Your scripts are very inspiring and often showed me entirely new programming approaches. I have learned a lot from you, although you might not be aware of that.

When I started my PhD journey, I wasn't the only one who was enrolled in the project. Being an early-stage researcher in a European training network, I was lucky to have a companion. Dear Giulia, thank you for being my buddy. I think the beginning of my PhD studies would have been way more difficult and I would have felt even more lost if there were not you. Thank you for all the talks we had, for sharing our happiness and frustration. Thank you for introducing me to the Italian culture, the fine differences in gestures and tastes. Under your influence I often caught myself using Italian words. Good luck with everything that is waiting ahead of you!

I was very lucky to share my office with two other PhD students: Dragan and Patrick. You both helped me a lot with finding the right way how to approach things, especially during my very first months in Maastricht. Good luck with your careers no matter where your future steps will lead you!

The very last person I shared my office with, before the coronavirus closed us at our homes, was Toni. Dear Toni, I'm so happy that our paths crossed. It's impossible to imagine the last year and a half in Maastricht without you, although a part of this time we could meet only via video calls. Good luck with your PhD studies in Verona!

Being a member of the research group, I was surrounded by great young investigators. Dear Billy and Eli, it was a pleasure to work with both of you. Thank you for all your help, especially with experiments. It was always fun to spent time with you and to watch your fights. I would also like to thank Ben, Joris, Martijn, Matthias and Piotr, great colleagues I always enjoyed talking to. Finally, I would like to wish a good luck to Ozan and Victor, the new talents of the group.

The experimental work on this thesis would have never been possible without Marion. Thank you so much for all your help and your support. I really appreciate it a lot! Being a 'highmaintenance' PhD student, I often took a lot of time from you. You were always thinking ahead and making sure that I don't forget something important. A big thank you also goes to Rachelle for all her help in the operating room as well as in the animal facility. I would also like to express my gratitude to Huub, Petra, Inger, Joyce, Richard and Saskia for taking great care of our animals. 
Dear Bianca, Jolanda and Vivian, thank you for all your support during my stay at the Department of Physiology. Your door was always open when I needed help, a suggestion or a piece of advice with various administrative as well as non-administrative issues.

My gratitude also goes to Ali for his warm welcome, help and hospitality during my stay at the Centre for Computational Medicine in Cardiology, Università della Svizzera italiana, Lugano, Switzerland.

Dear Nienke, Meike and Luuk, it was a pleasure meeting you. It was always really nice to stop by your office and have a chat. Thank you for all the hints and suggestions regarding everyday life in the Netherlands and good luck with your future endeavours!

My path towards $\mathrm{PhD}$ was exceptional thanks to being part of the international training network AFib-TrainNet. I would like to express my gratitude to Prof. Thomas Jespersen for setting up and organizing this network. The time I could spend together with the earlystage researchers of AFib-TrainNet definitely belongs to the happiest moments of my doctoral studies. Dear 'Afibbers', I feel honoured that I got the possibility to meet such excellent young people as you are. I am looking forward to seeing all of you again.

A large part of this thesis was written during the year of 2020. The very special times of a global pandemic resulted in safety measures that made our daily lives challenging in many different ways. Although 2020 is considered as "the worst year ever" by many, for me it was a very special year in the best sense of this word. It was a year when I got to know amazing people with whom I spent some beautiful and unforgettable moments. A big thanks therefore goes to my dear friends from Maastricht, my 'Maastricht family': Alex, Anxhela, Cristian, Delia, Isabella, Marco, Nico, Robin, Timo, Toni and everyone who was joining when the circumstances allowed it. Thank you for all your support and for being such great friends to me! I hope we will keep on seeing each other for a long long time!

Thinking about my life in Maastricht, it is difficult to imagine it without Radium Boulders, a place where we gathered once or twice a week and had a lot of fun when climbing interesting and often challenging boulders. It was also a place where I met many great climbing buddies. I would like to thank the members of the MosaMeat climbing crew Arianna, Johanna, Rada and Tobi for the nice bouldering sessions we had together. Also, I would like to thank Jorik and Shirley for the nice time we spent by climbing together at IVY in Sittard.

The final lines of this thesis were written in Bordeaux, France. My sincere thanks therefore go to my postdoctoral advisor Dr. Jason Bayer. Dear Jason, thank you so much for a warm 
welcome at IHU Liryc. I highly appreciate that you provided me with all the time I needed for making the final version of this thesis ready and for preparing my $\mathrm{PhD}$ defence. I am looking forward to continuing our collaboration.

$\mathrm{Na}$ závěr bych chtěl poděkovat svým rodičům, kteří mě podporovali po celou dobu mých studií. Velice si vážím toho, že jste mi umožnili jít za vším, pro co jsem se kdy nadchnul, a to i přesto, že ve vás moje nápady často vzbuzují více obav než nadšení. Poděkování za podporu patří také mému bratru Jaromírovi. I díky vám jsem svoje mnohaleté studijní snažení mohl zakončit doktorským titulem.

Final acknowledgment:

The author did not drink a single cup of coffee during his $\mathrm{PhD}$ studies. 


\section{ABOUT THE AUTHOR}

Vladimír Sobota was born on the $1^{\text {st }}$ of June 1990 in Vrchlabí, Czech Republic. He graduated from Gymnázium Jilemnice and in 2010 he enrolled at the Faculty of Biomedical Engineering, Czech Technical University in Prague, where he obtained both his bachelor's and master's degree. During his university studies he was a member of the Non-Conventional Ventilatory Team where he gained his first experience in scientific work. His main area of expertise at that time was the use of electrical impedance tomography for monitoring of lung ventilation. During the academic year 2014-2015 he was an Erasmus exchange student at the Faculty of Medicine, RWTH Aachen, Germany.

After obtaining his master's degree in 2016, he moved to Maastricht as a Marie SklodowskaCurie fellow within the ITN AFib-TrainNet (EU Training Network in Novel Targets and Methods in Atrial Fibrillation). There he worked as a $\mathrm{PhD}$ candidate at the Department of Physiology under the supervision of Prof. Ulrich Schotten and Dr. Sander Verheule. In his doctoral project he investigated the potential of new therapeutic targets for treatment of atrial fibrillation, particularly the role of the acetylcholine-activated potassium current. In addition, he also focused on development of new methods for analysis of mapping data of atrial fibrillation.

As of February 2021, he is a postdoctoral researcher at IHU Liryc, Bordeaux, France, where he works under the supervision of Dr. Jason Bayer on ERA-CVD project Sicvalves. In this project he uses computational models of human ventricles to better understand how adverse remodeling caused by aortic stenosis promotes ventricular arrhythmias. 


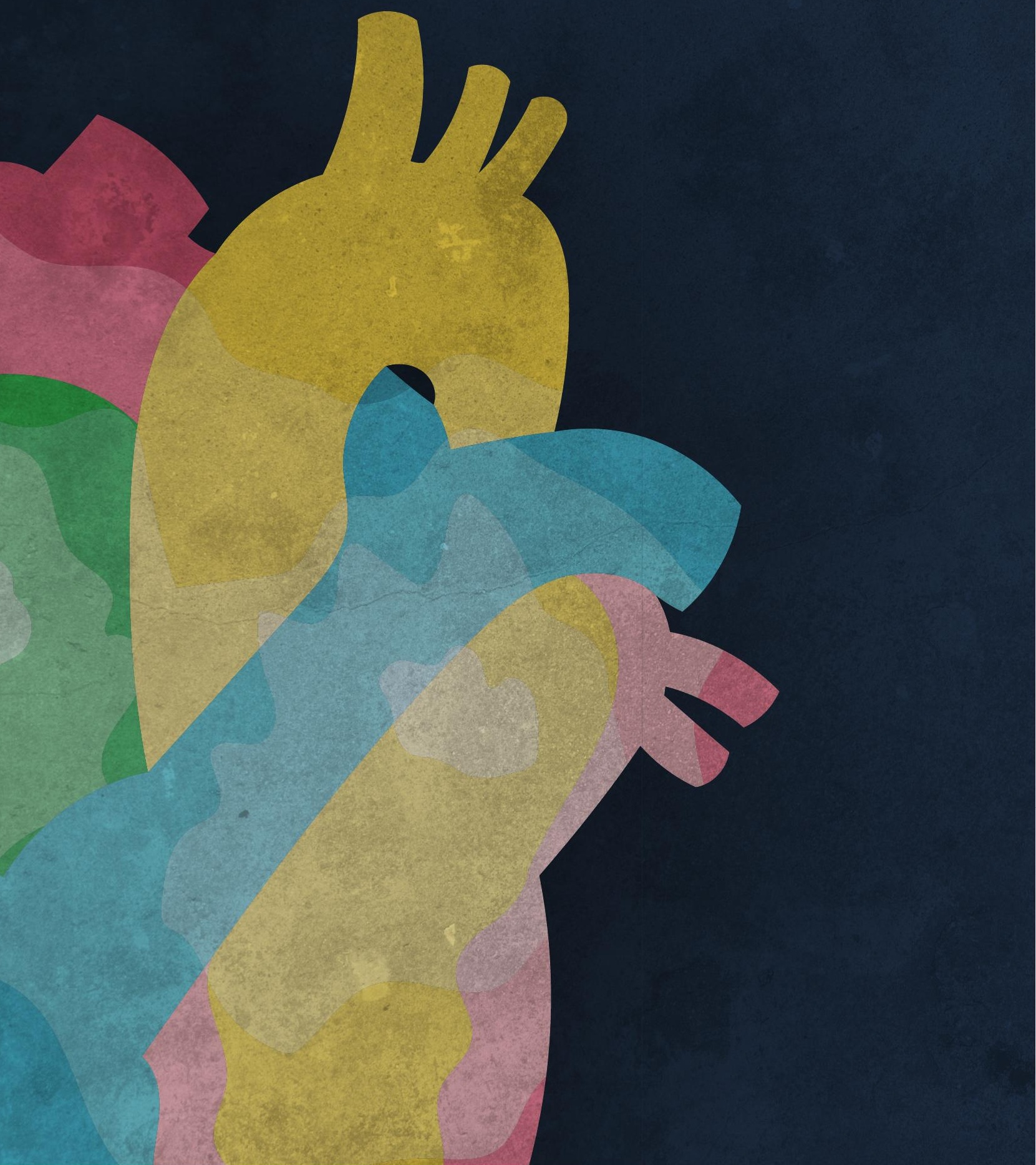

